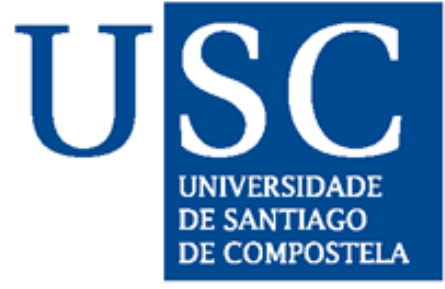

Tesis De Doctorado

\title{
Modelling systemic risk in financial markets
}

\author{
Andrea Ugolini \\ Programa DE DOCTORADO EN ECONOMÍA \\ FACUlTAD DE CiENCIAS ECONÓMICAS Y \\ EMPRESARIALES
}

Santiago de Compostela

2014 


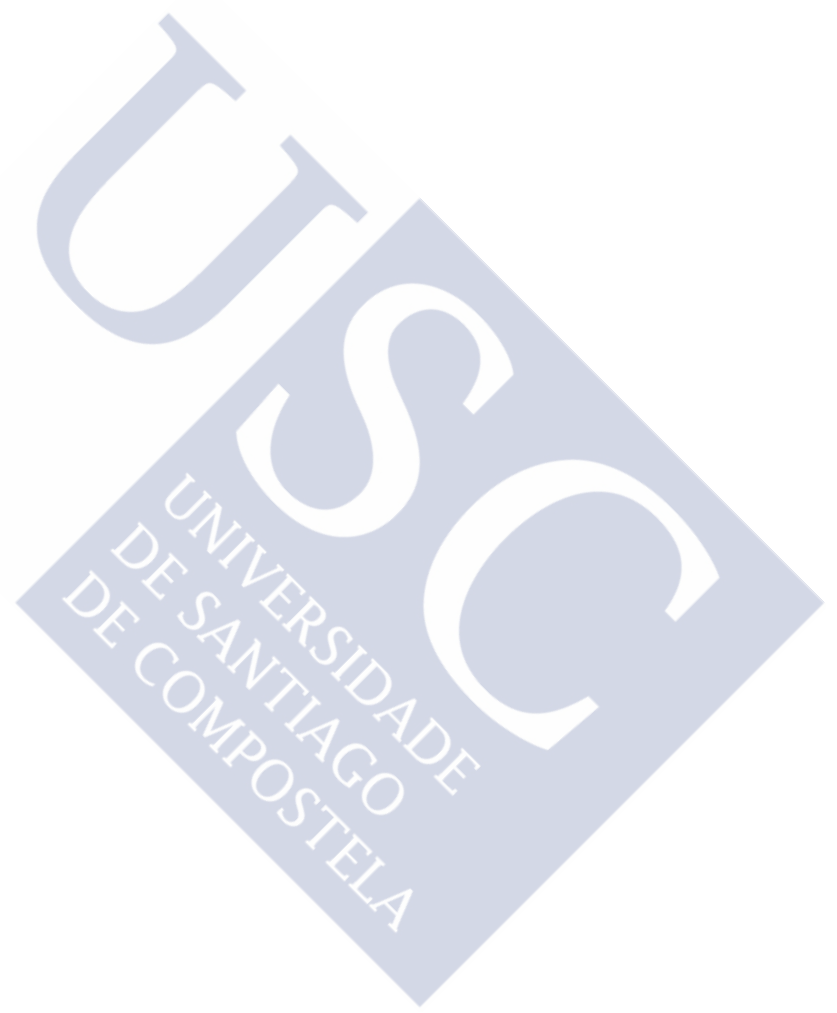




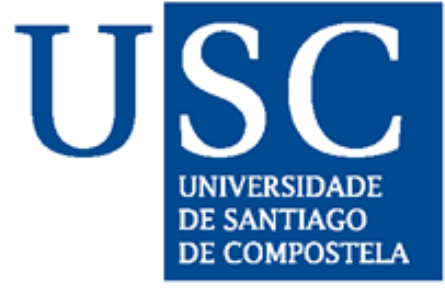

Tesis De Doctorado

\title{
Modelling systemic risk in financial markets
}

\author{
Asdo. \\ Andrea Ugolini \\ Programa DE DoCTORADO EN ECONOMÍA \\ FACUlTAd DE Ciencias ECONÓMICAS Y \\ EMPRESARIALES
}

Santiago de Compostela

September 2014 


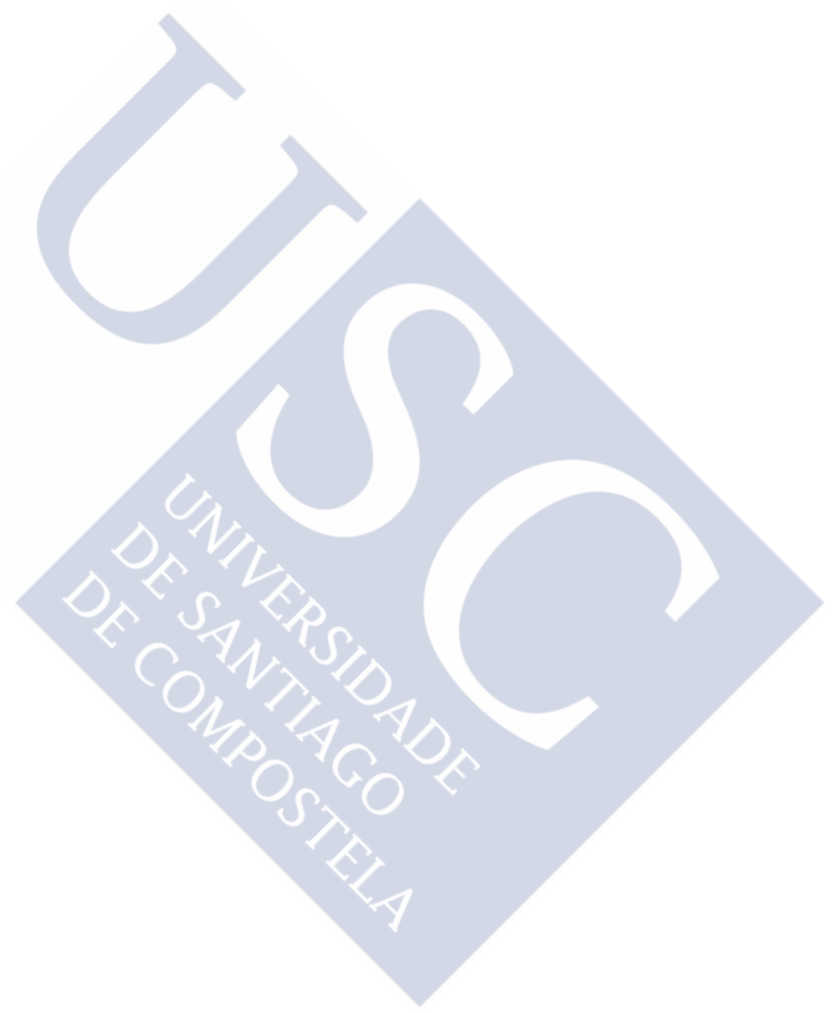




\section{AUTORIZACIÓN DO DIRECTOR/TUTOR DA TESI}

\section{Juan Carlos Reboredo Nogueira}

profesor do Departamento de Fundamentos de Análise Económica,

como Director da Tese de Doutoramento titulada «Modelling systemic risk in financial markets»

Presentada por D. Andrea Ugolini, alumno do Programa de Doutoramento en Economía:

Autorizo a presentación da tese indicada, cosiderando que reúne os requisitos esixidos no artigo 34 do regulamento de Estudos de Doutoramento, e que como Director da mesma non incurre nas causas de abstención establecidas na lei 30/1992

Asdo. 


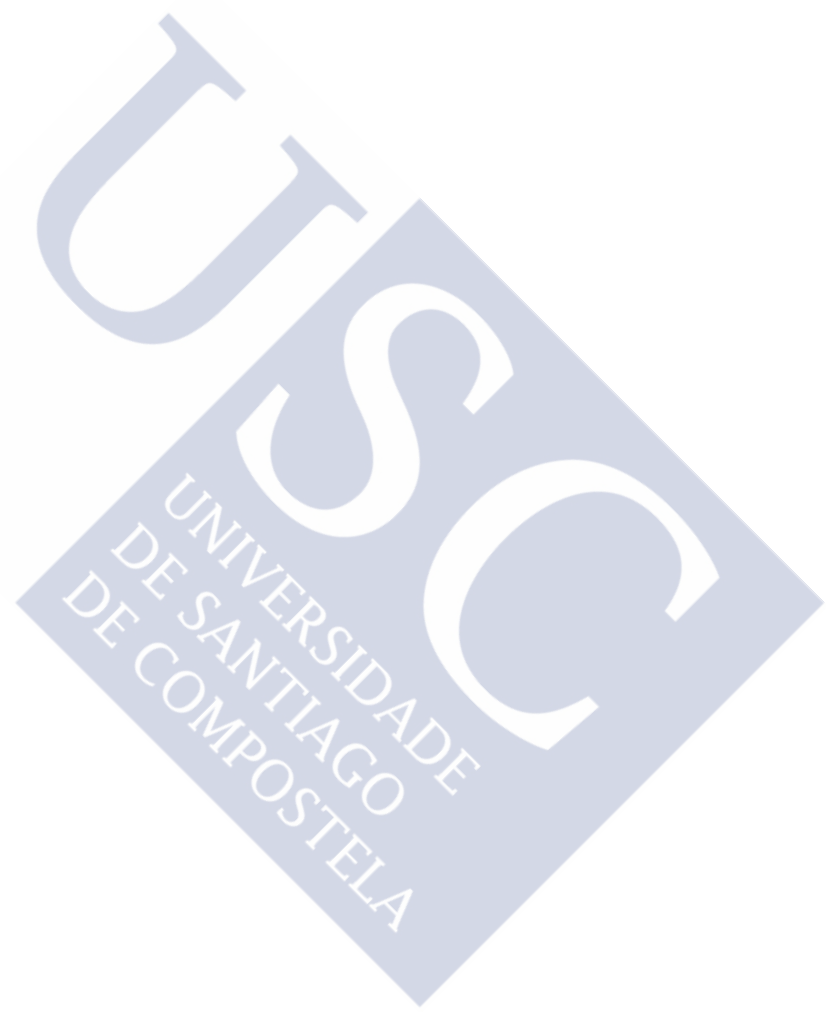




\section{Acknowledgements}

Giving thanks at the end of a personal and professional journey is an act of humility, to recognize the people that have helped me to achieve my goal. In this space, I have the opportunity to express my gratitude to people dearest to me, and thank them for their support throughout the course of this thesis.

First of all, I wish to express my profound gratitude to Professor Dr. D. Juan Carlos Reboredo Nogueira, without his expert guidance and help this thesis would not have been possible. I wish thank you for your openness and encouragement. It was a pleasure and an honour to work with such an inspirational, supportive, and insightful mentor. Your teachings sent me on scientific journeys of discovery and, on a personal level, how to live, love and deal with the world of research. I hope that this collaboration and friendship will endure for a long time.

I would not have contemplated studying abroad without the help and support of my dearest parents, Vincenzo and Lucia. Your love, encouragement and guidance throughout my life, has brought me to this stage of my life, culminating in the completion of this thesis. Without you, this thesis would not have be possible. You have made and continue to make sacrifices, so that I can realise my ambitions. For that, I will be eternally grateful. I could not be prouder to be you son.

To my Grandparents, Giuseppe and Teresa, thank you for your teachings and blessings that you have been giving me since I was young. You're my source of wisdom. You always refill my heart with joy when I return home. To my dearest aunt Angela, for being like a sister to me, thank you for your support.

A very special thanks to two people, who will forever remain close to my heart, aunt Elsa and uncle Marziano, for the love and confidence you gave me. Uncle Marziano, thank you for your phrase, which has been a source of inspiration through difficult times, "Ugo! you can do anything if you want it".

To my best friends, Andrea and Daniele, thank you for being awesome friends. Thank you for being there to listen. It's nice to know that even as time passes and the distance grows, interests changes our beautiful friendship will always remain.

I cannot forget to say thank you Daniel, Diana, Djamal, Fernando, Jesus, Maria, Óscar and Rafael. You were the perfect traveling companions, good colleagues and great friends. Fate could not have amassed a better group of people. 
To Marli, my girlfriend, who has always believed in me, motivated me, inspired me and supported me. She has withstood all the ups and downs with me. Without you, this thesis would not have been possible. You are a wonderful and incredible life partner, to whom I will be grateful forever.

Finally, thanks to the whole who of a form of other one has contributed to my enrichment as person. 


\section{Contents}

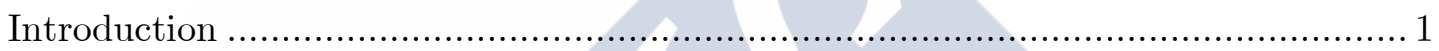

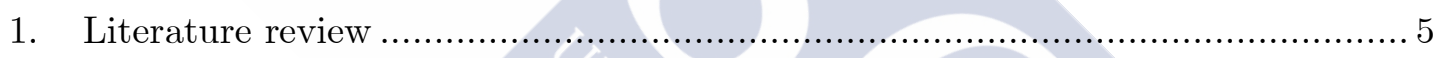

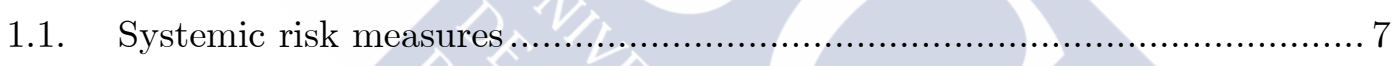

1.1.1. Mahalanobis distance ….......................................................... 7

1.1.2. Multivariate density estimators .................................................. 7

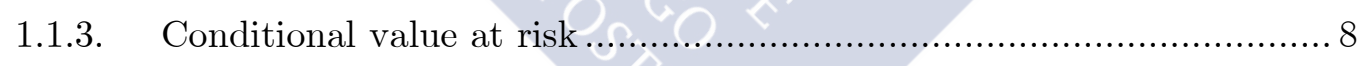

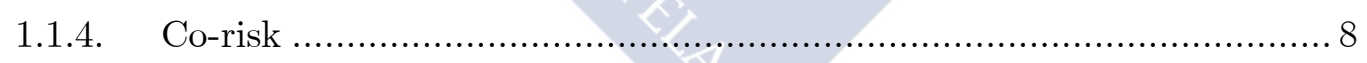

1.1.5. Systemic and marginal expected shortfalls..................................... 9

1.1.6. The default intensity model..................................................... 10

1.1.7. Distress insurance premium ................................................ 11

1.1.8. Broader hedge fund-based systemic risk measures .......................... 11

1.1.9. Granger causality tests ........................................................ 12

1.1.10. Simulating a credit scenario ..................................................... 12

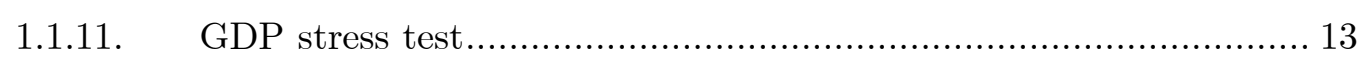

2. Measuring systemic risk in the Spanish financial system: A CoVaR approach. 15

2.1. Introduction 15 


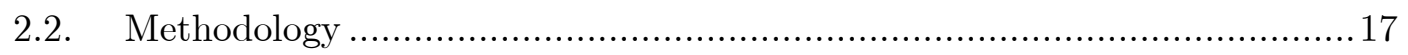

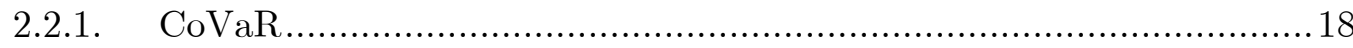

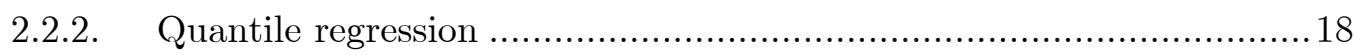

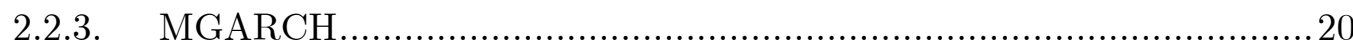

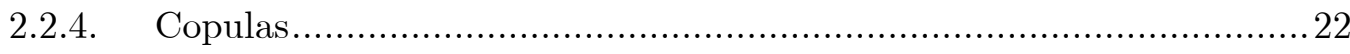

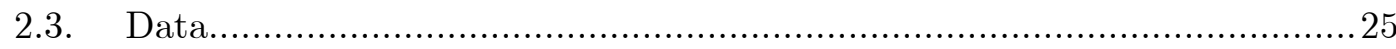

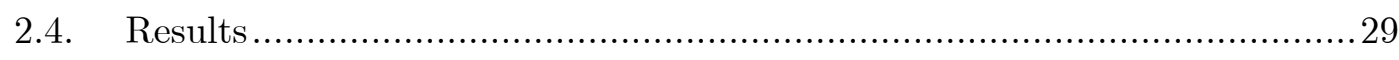

2.4.1. Marginal model and VaR results................................................29

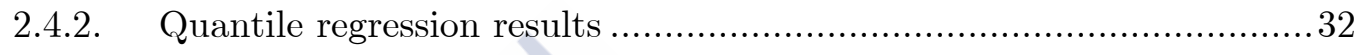

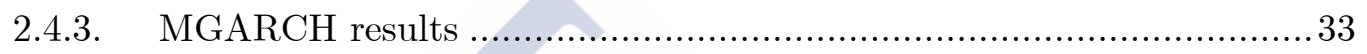

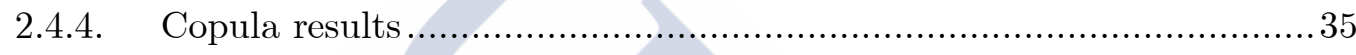

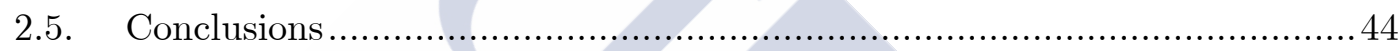

3. Systemic risk in European sovereign debt markets: A CoVaR-copula approach 47

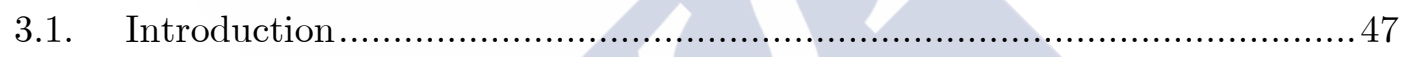

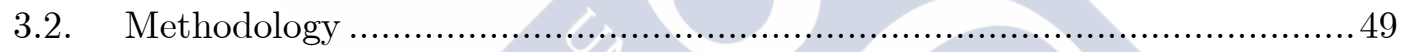

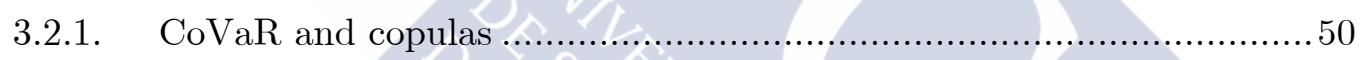

3.2.2. Marginal distribution and copula models ....................................5 52

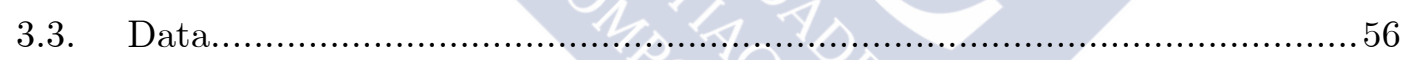

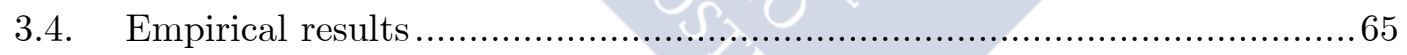

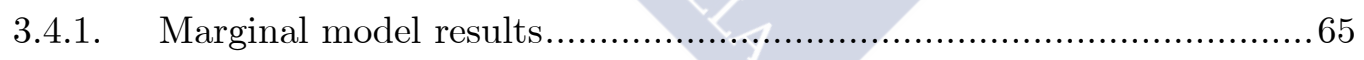

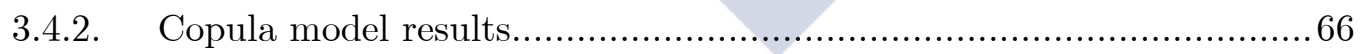

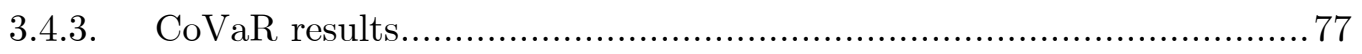

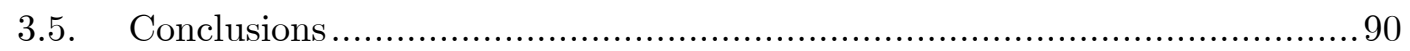

4. A Vine-copula CoVaR approach to systemic sovereign debt risk for the financial

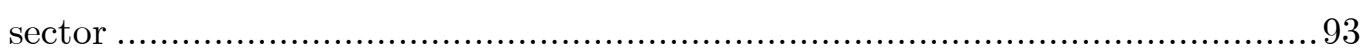

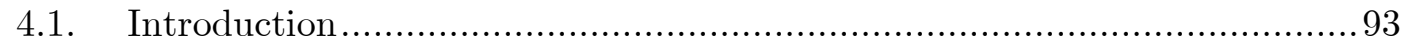

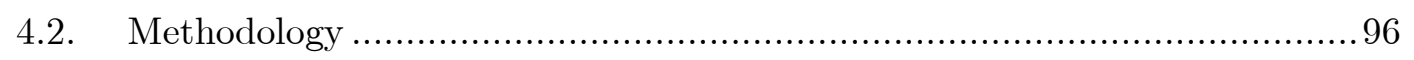

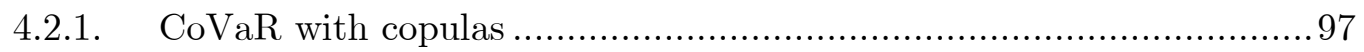

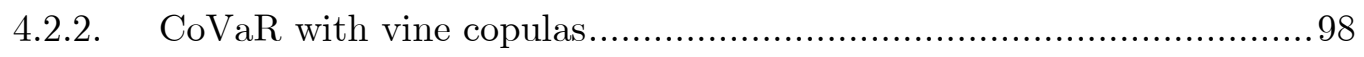


4.2.3. Marginal distribution and copula models......................................100

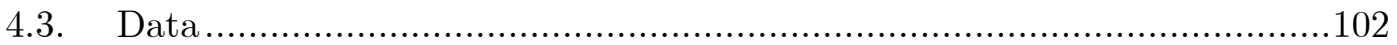

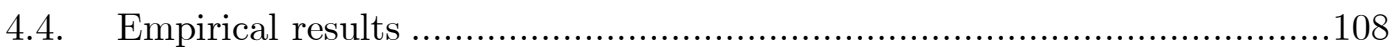

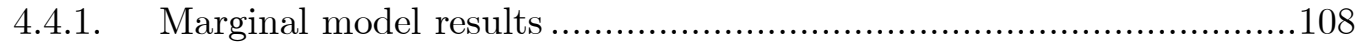

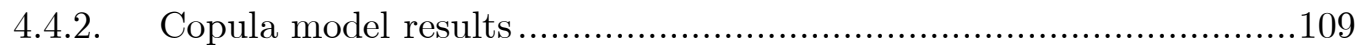

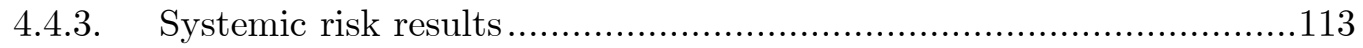

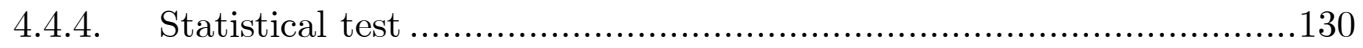

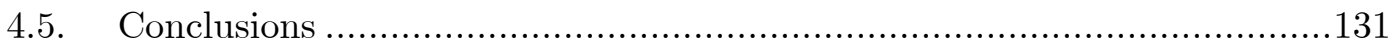

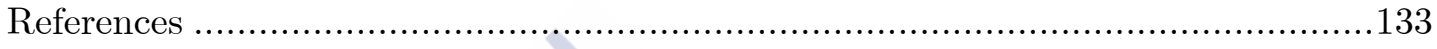

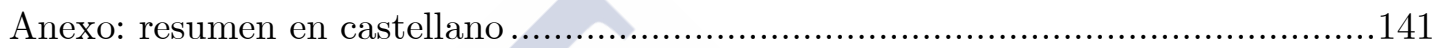




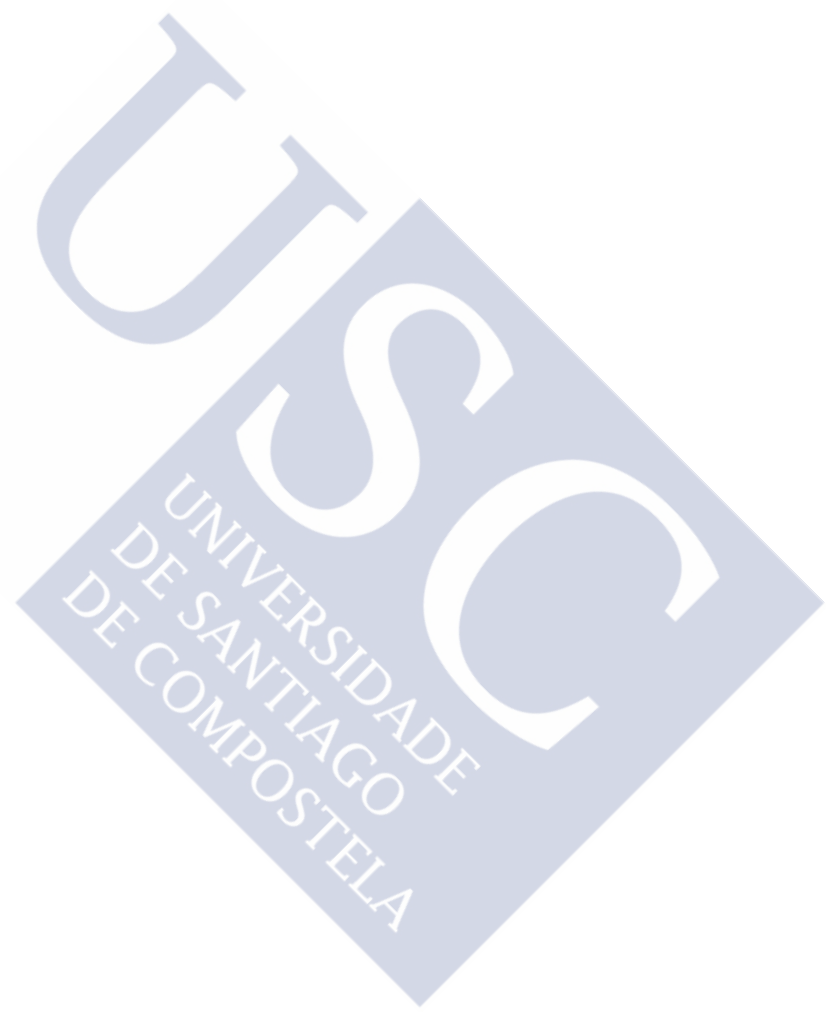




\section{List of Figures}

Figure 2.1: Time series plot of daily returns for each institution and the MSCI

Index 26

Figure 2.2: Time series plot for the explanatory variables................................. 27

Figure 2.3: Time series plot for value-at-risk at the $95 \%$ confidence level.............. 32

Figure 2.4: Time series plot for dynamic conditional correlation (DCC) ................ 34

Figure 2.5: Estimates of CoVaR (left axis) and $\triangle \mathrm{CoVaR}$ (right axis) for the MSCI

Index with respect to each institution. ............................................. 38

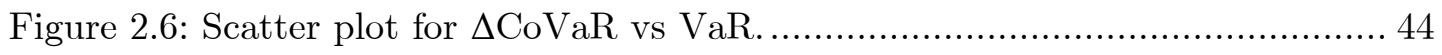

Figure 3.1: Time series plot of weekly bond returns for selected countries and the European Economic and Monetary Union (EMU)............................ 58

Figure 3.2: Time series plot of weekly market index returns for indices for selected countries and the European Economic and Monetary Union (EMU)...6 60

Figure 3.3: Time series plot of weekly market volatility returns for selected countries and the Eurostoxx 50.

Figure 3.4: $\mathrm{CoVaR}$ (left axis) and $\Delta \mathrm{CoVaR}$ (right axis) estimates for the European Economic and Monetary Union (EMU) with respect to selected countries.

Figure 3.5: CoVaR (left axis) and $\Delta \mathrm{CoVaR}$ (right axis) estimates for Greece with respect to selected countries.

Figure 4.1: A D-Vine copula hierarchical structure ............................................ 99

Figure 4.2: Time series plot of weekly sovereign bond price indices. ......................105

Figure 4.3: Time series plot of weekly MSCI financial price indices. ......................106

Figure 4.4: Times series plots for parameter estimates of the best copula fits between domestic financial systems and sovereign debt returns.

Figure 4.5: Times series plots for parameter estimates of the best copula fits between domestic financial systems and sovereign Greek debt returns....

Figure 4.6: Times series plots for parameter estimates of the best copula fits between domestic sovereign and Greek sovereign debt returns.

Figure 4.7: Times series plots for parameter estimates of the best copula fits between domestic financial systems and domestic sovereign debt returns conditional on sovereign Greek debt returns.

Figure 4.8: $\operatorname{VaR}(\mathrm{f})$ and $\operatorname{CoVaR}(\mathrm{f} \mid \mathrm{d})$

Figure 4.9: Results for $\operatorname{CoVaR}\left(\mathrm{f} \mid \mathrm{d}=0.05, \mathrm{~d}^{*}\right), \operatorname{CoVaR}\left(\mathrm{f} \mid \mathrm{d}, \mathrm{d}^{*}=0.05\right)$ and $\mathrm{CoVaR}$

$$
\left(\mathrm{f} \mid \mathrm{d}=0.05, \mathrm{~d}^{*}=0.05\right) \text {. }
$$




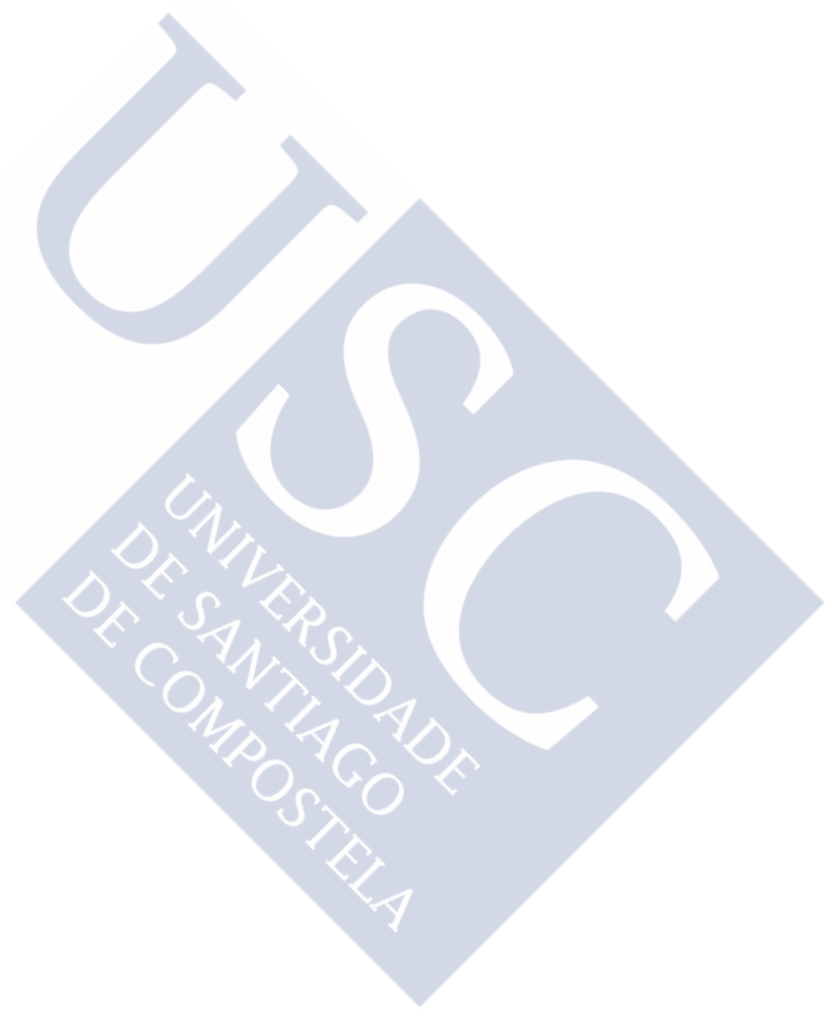




\section{List of Tables}

Table 2.1: Copula specifications.

Table 2.2: Descriptive statistics for weekly price returns for selected Spanish

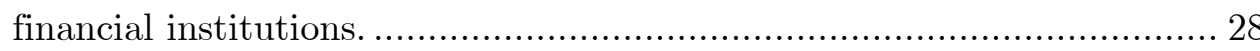

Table 2.3: Descriptive statistics for specific risk factors. .................................. 27

Table 2.4: Maximum likelihood estimates.............................................................. 30

Table 2.5: Descriptive statistics for the value-at-risk at the $95 \%$ confidence level... 31

Table 2.6: Quantile regression estimates at the $95 \%$ confidence level...................... 33

Table 2.7: Dynamic conditional correlation (DCC) model estimates...................... 34

Table 2.8: Copula model estimates for institutions vs the MSCI Index................... 36

Table 2.9: Summary statistics for CoVaR-QR, CoVaR-Student-t, CoVaR-Copula and $\Delta \mathrm{CoVaR}$ - Copula for each institution with the MSCI Index.......... 43

Table 3.1: Descriptive statistics for sovereign bond price returns......................... 59

Table 3.2: Descriptive statistics for stock market returns. ................................. 63

Table 3.3: Descriptive statistics for market volatility and Euribor at 1 year........... 64

Table 3.4: Pearson correlation coefficients........................................................ 65

Table 3.5: Parameter estimates for the marginal distribution models. .................... 68

Table 3.6: Estimates for copula models in the period before crisis onset. European Economic and Monetary Union (EMU) vs. selected countries.

Table 3.7: Estimates for copula models in the period after crisis onset. European

Economic and Monetary Union (EMU) vs. selected countries 71

Table 3.8: Estimates for the copula models in the period before crisis onset. Greece

vs. country

Table 3.9: Estimates for the copula models in the period after crisis onset. Greece vs. country 75

Table 3.10: Summary CoVaR and ${ }_{\Delta} \mathrm{CoVaR}$ statistics for the European Economic and Monetary Union (EMU) and selected countries.

Table 3.11: Summary CoVaR statistics for selected European debt markets and Greece. 86

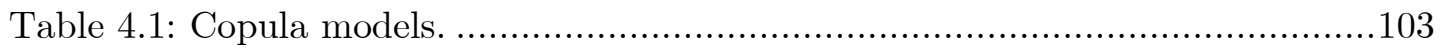

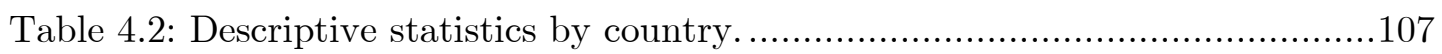

Table 4.3: Pearson correlation between financial returns and sovereign debt returns by country. 108

Table 4.4: Marginal distribution model. Parameter estimates for sovereign debt returns by country. 
Table 4.5: Marginal distribution model. Parameter estimates for MSCI financial index returns by country............................................................ 111

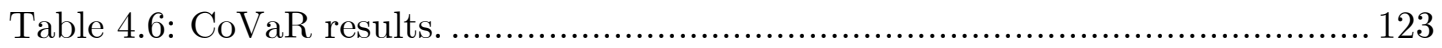

Table 4.7: Significant test for differences in risk measures.................................... 131 


\section{Introduction}

Three recent crises - the dot-com bubble and the subprime and European sovereign debt crises - have revealed the complex dynamics underpinning the global financial system and how rapidly risk is propagated across markets. Investors, regulators and researchers are thus keen to develop accurate measures of risk transmission between assets and markets. From the investors' perspective of guaranteeing efficient portfolio diversification, the risk of contagion is essential for their ongoing interest in changes in market linkages. From the regulators' point of view, risk spillover is important to focalize attention on the maintenance and development of new financial regulatory and institutional rules such as circuit breakers, transaction taxes and short-sale rules. In fact, recognizing important shortcomings in financial supervision, the European Commission and European Central Bank (ECB) created the European Systemic Risk Board (ESRB) at the end of 2010 with the goal of monitoring, at the macro-prudential level, the European financial system and preventing and mitigating any propagation risk within the financial system.

The literature contains many definitions of systemic risk. De Bandt and Hartmann (2000) defined it "as the risk of experiencing systemic events in the strong sense" where "strong sense" signifies the spread of news about an institution that has an adverse impact on one or more healthy institutions in a sequential manner. Furfine (2003) distinguished between two kinds of systemic risk: "The first type is the risk that some financial shock causes a set of markets or institutions to simultaneously fail to function efficiently. The second type of systemic is the risk that failure of one or a small number of institutions will be transmitted to others due to explicit financial linkages across institutions". On the basis of the theoretical model proposed by Diamond and Dybvig (1983), Acharya (2009) defined systemic risk "as the joint failure risk arising from correlation of returns on the asset-side of 
bank balance-sheets". Billio et al. (2010) explained that "systemic risk can be realized as a series of correlated defaults among financial institutions, occurring over a short time span and triggering a withdrawal of liquidity and widespread loss of confidence in the financial system as a whole". The last two definitions introduce the notion that common investment between banks generates correlation and "herding effects", thereby generating systemic risk. The International Monetary Fund (IMF), Bank for International Settlements (BIS), Financial Stability Board (FSB) and the ECB focus their attention particularly on the consequences of systemic risk for the real economy. Thus the IMF, BIS, FSB (2009) stated systemic risk to be "the disruption to the flow of financial services that (1) is caused by an impairment of all or parts of the financial system; and (2) has the potential to have serious negative consequence for the real economy". The ECB (2009) conceptualized systemic risk as a "risk that financial instability becomes so widespread that it impairs the functioning of the financial system to the point where economic growth and welfare suffer materially". The above brief list of definitions points to the intricacy of the topic and the challenge faced by investors, regulators and researchers in attempting to measure the complexity and dynamics of systemic risk.

Using the CoVaR systemic risk measure (Adrian and Brunnermeier, 2011; Girardi and Ergün, 2013), we quantified systemic risk as the impact of the risky situation of a particular financial institution, market or system on the value-at-risk (VaR) of other financial institutions, markets or systems. Our research objectives potentially of interest to investors, regulators and researchers in equal measure were as follows:

1. To quantify systemic risk for Spanish financial institution and to account for the quantitative effect on the conditional VaR value with a view to assessing how the fragile position of one particular financial institution could impair the performance of other financial institutions and to determine how much regulatory capital a financial institution would need in order to cover its exposure to this kind of risk.

2. To examine systemic risk in European sovereign debt markets and assess how this risk changed with the onset of the recent European sovereign debt crisis with a view to determining how the deteriorated financial position of a particular sovereign debt market can impair the performance of other sovereign debt markets. 
3. To measure the systemic impact of domestic sovereign debt distress on domestic financial systems in Europe and of a potentially distressed Greek debt market on the financial systems of other countries during the recent financial and debt crises with a view to understanding the impact of domestic sovereign distress on a domestic financial system and the impact of Greek sovereign debt distress on other financial systems.

The dissertation is laid out as follows:

In Chapter 1 we provide a survey of the quantitative measure of systemic risk in the economics and finance literature.

In Chapter 2 we examine, using conditional $\mathrm{VaR}(\mathrm{CoVaR})$, the systemic risk generated by major Spanish financial institutions in the recent global financial crisis and the European sovereign debt crisis as a systemic risk measure. CoVaR was quantified using quantile regression, multivariate generalized autoregressive conditional heteroskedasticity (MGARCH) and copula approaches. We also describe a novel copula-based approach to computing the CoVaR value, given that copula are flexible modellers of joint distribution and are particularly useful for characterizing the tail behaviour that provides such crucial information for the CoVaR. We found significant increases in systemic risk around the time of the recent global financial crisis and, to a lesser extent, around the time of the European debt crisis. Our evidence also shows that the quantile regression approach was unable to reflect the dynamics of, and sudden changes in, systemic risk. These results have implications for capital regulation in financial institutions and on how systemic risk should be measured.

In chapter 3 we study systemic risk in European sovereign debt markets before and after the onset of the Greek debt crisis, taking, as a systemic risk measure, the CoVaR as characterized and computed using copulas. We found sovereign debt markets to be coupled before the debt crisis and systemic risk to be similar for all countries. With the onset of the Greek crisis, debt markets decoupled and the systemic risk of the countries in crisis (excepting Spain) decreased whereas that of the non-crisis countries increased slightly. The systemic risk of the Greek debt market increased for other countries in crisis, especially for Portugal (where systemic risk tripled after the onset of the crisis) and decreased for non-crisis countries.

In Chapter 4 we investigated - using the CoVaR measure as characterized and computed using copulas and vine copulas - systemic sovereign debt distress in European domestic financial systems and the systemic risk of a potentially distressed 
Greek debt market for other European financial systems countries before and after the onset of the recent financial and debt crises. We found that, before the debt crisis, sovereign debt had a positive systemic risk on European domestic financial systems. However, with the onset of the Greek crisis, the systemic impact of sovereign debt increased for countries in crisis (Greece, Italy and Portugal) whereas it remained stable or reduced for non-crisis countries. Regarding the systemic impact of sovereign Greek debt distress, our evidence indicates that negative impacts were limited to a small set of countries (Belgium, Italy, the Netherlands and Portugal).

The dissertation results are described in three research articles with following titles:

1. A CoVaR approach to systemic risk in the Spanish financial system

2. A CoVaR-copula approach to systemic risk in European sovereign debt markets

3. A vine copula-CoVaR approach to systemic sovereign debt risk for the financial sector.

The realization of this results was only feasible by accessing to database as Bloomberg or Datastream. Furthermore, with the use of computer programs as: Matlab, R-Project, Eviews and OxMetrics. 


\section{Chapter 1}

\section{Literature review}

Over the last years, researchers have developed a number of systemic risk measures referring to different systemic risk propagation channels. Three main measures can be identified: (1) measures of systemic risk that capture the contagion and exposure effect between institutions; (2) measures of systemic risk that quantify the trigger effect between the financial sector and the real economy; and (3) measures of systemic risk between the financial and public sectors and vice versa.

The first category refers to the risk of a failure of one financial institution having a contagion or domino effect on other institutions through the transactions and interconnections linking these institutions. Many researchers have focused their attention on this kind of risk propagation. Segoviano and Goodhart (2009) created a banking stability index that assesses interbank dependence for tail events. Acharya et al. (2010) used systemic and marginal expected shortfall (ES) measures to quantify downside risk and the contribution of individual financial institutions to risk. Allen et al. (2010) proposed a measure of aggregate systemic risk - called CATFIN - to predict declines in aggregate bank lending activity six months in advance. Huang, Zhou and Zhu (2009, 2010, 2012) proposed their distress insurance premium (DIP) measure. Adrian and Brunnermeier (2011) proposed using conditional value-at-risk (CoVaR) to capture possible risk spillovers between financial institutions. Likewise, Brownless and Engle (2012) developed a systemic risk measure, called SRISK, to represent the amount of capital needed to restore minimum capital requirements. Billio et al. (2012) proposed five measures of 
systemic risk to capture contagion and exposure effects in the relationship between financial institutions. Girardi and Ergün (2013) proposed a new approach to quantifying $\mathrm{CoVaR}$ using the joint density for the financial system and financial institution returns. Finally, Halaj et al. (2013) suggested a simple network analysis measure, called the systemic probability index (SPI).

Regarding measures of the trigger effect, several authors have developed a systemic risk measure based on interdependence between the financial sector and the real economy. Reinhart and Rogoff (2009a) showed that systemic risk in financial markets increases in crisis periods and has adverse effects that extend to the real economy. Giesecke and Kim (2011) developed the default intensity model (DIM) to capture spillover effects through the complex network of relationships with the real economy. De Nicolò and Lucchetta (2010) proposed a GDP-at-risk model to quantify the impact between the macro-economy, the financial markets and intermediaries.

Finally, regarding systemic risk generated between the financial and public sectors, Reinhart and Rogoff (2009b, 2010) documented sovereign distress spread to the financial system when banks held a substantial amount of government debt in their portfolio. Alter and Schuler (2012) examined the relationship between sovereign default risk and domestic banks. Mink and De Haan (2013) analysed the impact of highly volatile Greek bonds on European bank stock prices in 2010 and De Bruyckere et al. (2013) studied contagion between banking and sovereign default risk in Europe through asset, collateral and rating channels. Bhanot et al. (2014) investigated the impact of changes in Greek sovereign yield spreads on stock returns in the financial sector. Battistini et al. (2014) demonstrated that the sovereign debt portfolios of European banks revealed growing home bias during the recent crisis, with domestic sovereign debt holdings growing in line with sovereign solvency risk.

Finally, alternative risk measures have been proposed, other than those included in the three categories mentioned above. Engle and Manganelli (2004) developed their conditional autoregressive value at risk (CAViaR) model that uses quantile regression to capture the tail behaviour of returns. De Jonghe (2010) used extreme value theory to measure banks' systemic risk exposure. Zhou (2010) used multivariate extreme value theory to quantify systemic risk, analysing the relationship between institution size and systemic importance. Finally, Krizman et al. (2011) developed a measure of systemic risk called the absorption ratio that relies on principal component analysis (PCA). 


\subsection{Systemic risk measures}

Below we describe the methodologies used to quantify systemic risk measures. In the selection of the systemic risk measures, we would trace briefly all aspects that the literature aims to address.

\subsubsection{Mahalanobis distance}

Kritzman and Li (2010) defined "financial turbulence" as the statistical unusualness of a set of returns given historical behaviour patterns, including extreme price movements, decoupling of correlated assets and convergence of uncorrelated assets. To quantify turbulence they used the Mahalonabis distance (Mahalanobis, 1927). Given the returns for a particular period of $n$ assets, the turbulence index was formally defined as:

$$
d_{t}=\left(y_{t}-\mu\right)^{\prime} \Sigma^{-1}\left(y_{t}-\mu\right) \text {, }
$$

where:

$$
\begin{aligned}
& d_{t}=\text { turbulence at time } t \\
& y_{t}=\text { vector of asset returns at time } t(n \times 1) \\
& \mu=\text { sample average vector of historical returns }(n \times 1) \\
& \Sigma=\text { sample covariance matrix of historical returns }(n \times n)
\end{aligned}
$$

When applying the turbulence index to two assets, we consider the difference between the return for the first asset at time $t$ and the mean return and the difference between the return for the second asset at time $t$ and the mean return and calculate the covariance between the returns for these two assets. We then take the absolute value of the final calculation so that the turbulence index is always positive. The information provided by the financial turbulence index is helpful because assets that may be negatively correlated during normal economic conditions may become positively correlated during times of high turbulence. This systemic risk measure, which can be used for stress tests of asset portfolios, provides a realistic estimate of possible losses arising from a systemic event.

\subsubsection{Multivariate density estimators}

Segoviano and Goodhart (2009) developed a systemic risk measure based on the banking system's multivariate density (BSMD). Considering the banking system as 
a portfolio of banks, with each bank as part of the portfolio, probabilities of distress can be obtained by estimating the BSMD using a multivariate density methodology (Segoviano, 2006). It is also possible to estimate banking stability measures from the BSMD. Segoviano and Goodhart (2012) used this methodology to examine and quantify relative changes in stability over time in the following cases: (1) general distress in the banking system; (2) distress between specific banks; and (3) distress in the system associated with a specific bank. Given the BSMD, the authors proposed a set of measures of systemic risk, namely, joint probability of default, the banking stability index and the distress dependence matrix.

\subsubsection{Conditional value at risk}

Adrian and Brunnermeier (2011) proposed the CoVaR measure for systemic risk, which captures possible risk spillovers between financial institutions by providing information on the $\mathrm{VaR}$ of the financial system conditional on the fact that a financial institution is in distress. These authors also calculated the systemic risk contribution of an institution as the $\triangle \mathrm{CoVaR}$, which measures the difference between the CoVaR under financial distress and the CoVaR in the benchmark state. Formally, the CoVaR can be defined as the q-quantile of a conditional distribution:

$$
\operatorname{Pr}\left(\mathrm{X}^{j} \leq \operatorname{CoVaR}_{\mathrm{q}}^{\mathrm{j} \mid \mathrm{i}} \mid \mathrm{X}^{i}=\operatorname{VaR}_{\mathrm{q}}^{\mathrm{i}}\right)=\mathrm{q}
$$

with the systemic risk contribution defined as:

$$
\Delta \mathrm{CoVaR}_{\mathrm{q}}^{\mathrm{j} \mid \mathrm{i}}=\mathrm{CoVaR}_{\mathrm{q}}^{\mathrm{j} \mid \mathrm{i}}-\mathrm{CoVaR}_{50 \%}^{\mathrm{j} \mid \mathrm{i}} .
$$

The authors proposed using quantile regression to compute the CoVaR:

$$
\operatorname{CoVaR}_{t}^{i}=\alpha^{\text {system } \mid i}+\beta^{\text {system } \mid i} \operatorname{VaR}_{t}^{i}+\gamma^{\text {system } \mid i} M_{t-1},
$$

where $M_{t-1}$ denotes a set of explanatory variables.

\subsubsection{Co-risk}

An IMF Global Financial Stability Report (IMF, 2009a) proposed the co-risk methodology to estimate co-movements between the credit default swap (CDS) spreads for several financial institutions. This methodology assesses direct and indirect financial linkages that may arise from exposure to common risks (similar business models, common accounting practices, etc). Like the CoVaR, co-risk 
employs quantile regression to estimate co-movement between risk factors for financial institutions in distress. The co-risk measure is formally defined as:

$$
\mathrm{CDS}_{\mathrm{i}}=\alpha_{\tau}+\sum_{\mathrm{i}}^{\mathrm{k}} \beta_{\tau, \mathrm{i}} \mathrm{R}_{\mathrm{i}}+\beta_{\tau, \mathrm{i}} \mathrm{CDS}_{\mathrm{j}}
$$

where $\mathrm{CDS}_{\mathrm{i}}$ is the credit default swap spread of the institution $\mathrm{i}, \mathrm{CDS}_{\mathrm{j}}$ is the credit default swap spread of the institution $\mathrm{j}, \mathrm{R}_{\mathrm{i}}$ denotes common aggregated risk factors, $\tau$ denotes the quantile (usually the $95^{\text {th }}$ ) corresponding to a distress period and where $\beta_{\tau, \mathrm{i}}$ are the parameter estimates that quantify the input of firms as the credit risk of firm i at quantile $\tau$. Hence, the conditional co-risk measure is given by:

$$
\operatorname{CoRisk}_{\mathrm{i}, \mathrm{j}}=100 \times\left(\frac{\alpha_{95}+\sum_{\mathrm{i}}^{\mathrm{k}} \beta_{95, \mathrm{i}} \mathrm{R}_{\mathrm{i}}+\beta_{95, \mathrm{j}} \mathrm{CDS}_{\mathrm{j}}(95)}{\operatorname{CDS}_{\mathrm{i}}(95)}-1\right) \text {, }
$$

where $\operatorname{CDS}_{\mathrm{i}}(95)$ and $\mathrm{CDS}_{\mathrm{j}}(95)$ are the CDS spreads of institution $i$ and $j$ corresponding to the $95^{\text {th }}$ percentile and where $\alpha_{95}, \beta_{95, \mathrm{i}}$ and $\beta_{95, \mathrm{j}}$ are the quantile regression parameters at the $95^{\text {th }}$ level.

\subsubsection{Systemic and marginal expected shortfalls}

Acharya et al. (2010) showed how a financial institution's contribution to systemic risk could be measured and priced as the systemic expected shortfall (SES). The SES represents a propensity to be undercapitalized when the system as a whole is undercapitalized. The SES can be quantified using three measures: (1) the outcome of stress tests performed by regulatory bodies; (2) the decline in equity valuations of large financial firms during a crisis; and (3) the widening of the credit default swap spread of large financial institutions. The same authors also developed leading indicators denominated the marginal expected shortfall (MES) and leverage (LVG) defined as the ratio of the quasi-market value of assets market to the value of equity. They define the MES of a financial institution as its short-run expected equity loss conditional on the market taking a loss greater than its $\mathrm{VaR}$ at $\alpha \%$. Formally it is expressed as:

$$
\operatorname{MES}_{\mathrm{i}, \mathrm{t}}=E\left(\mathrm{r}_{\mathrm{i}, \mathrm{t}+1} \mid \mathrm{r}_{\mathrm{m}, \mathrm{t}+1}<\mathrm{q}_{\alpha, \mathrm{t}}\left(\mathrm{r}_{\mathrm{t}+1}\right)=\mathrm{C}\right),
$$

or 


$$
\operatorname{MES}_{\mathrm{i}, \mathrm{t}}=E\left(\mathrm{r}_{\mathrm{i}, \mathrm{t}+1} \mid \mathrm{r}_{\mathrm{m}, \mathrm{t}+1}<\mathrm{C}\right),
$$

where $r_{i, t}$ is the stock return of the institution, $r_{m, t}$ is the market return and $C$ is a constant corresponding to what is considered to be "tail risk" in the market. As all the considered institutions belong in the market, it is straightforward to see that the MES of one institution is simply the derivate of the market ES with respect to the institution's market share (or capitalization), hence the term "marginal". LVG is defined as:

$$
\mathrm{LVG}_{\mathrm{i}}=\frac{\text { quasi-market value of assets }}{\text { market value of equity }} .
$$

Moreover, applying cross-section regression analyses of firms' SES on MES and LVG:

$$
\mathrm{SES}_{\mathrm{i}}=\alpha+\beta \mathrm{MES}_{\mathrm{i}}+\gamma \mathrm{LVG}_{\mathrm{i}}+\varepsilon_{\mathrm{i}}
$$

After estimating the three parameters, $\alpha, \beta$ and $\gamma$, for specific SES metrics, the systemic risk of financial institution at a future time $t$ can be calculated as:

$$
\text { Systemic Risk of Firm } i=\frac{\hat{\beta}}{\hat{\beta}+\hat{\gamma}} M E S_{i}^{t}+\frac{\hat{\gamma}}{\hat{\beta}+\hat{\gamma}} L V G_{i}^{t} \text {. }
$$

\subsubsection{The default intensity model}

Giesecke and Kim (2011) developed the default intensity model (DIM) as a dynamic measure of the systemic risk of the financial sector as a whole. Recognizing that systemic distress involves all economic agents, they proposed considering the systemic risk of the financial sector as the conditional probability of the failure of most financial institutions. The model estimated in terms of hazard rate, or intensity, is expressed as:

$$
\lambda_{t}^{*}=\exp \left(\beta^{*} X_{t}^{*}\right)+\int_{0}^{t} e^{-\kappa(t-s)} d J_{s}
$$

where $X_{t}^{*}$ is a time-varying vector of the several explanatory variables, $\beta^{*}$ is a vector of parameters and

$$
\mathrm{J}_{\mathrm{t}}=\mathrm{v}_{1}+\ldots \mathrm{v}_{\mathrm{N}_{\mathrm{t}}^{*}}
$$


where $\mathrm{v}_{\mathrm{n}}=\gamma+\delta \max \left(0, \log \mathrm{D}_{\mathrm{n}}^{*}\right)$. Intensity is the sum of two terms with a bearing on economy-wide default: baseline hazard and spillover hazard. The model's parameters are obtained by maximizing log-likelihood for the default rate as follows:

$$
\max _{\theta \in \Theta} \int_{0}^{\mathrm{t}}\left(\log \lambda_{\mathrm{s}-}^{*}(\theta) d \mathrm{~N}_{\mathrm{s}}^{*}-\lambda_{\mathrm{s}}^{*}(\theta) \mathrm{ds}\right) .
$$

The conditional distribution at time $t$ of economy-wide defaults over the period is estimated via Monte Carlo simulation. Finally, $\mathrm{VaR}$ at level $\alpha$ is used to quantify the systemic risk. DIM is thus able to capture default spillover within and between sectors and also can provide accurate out-of-sample forecasts of systemic risk.

\subsubsection{Distress insurance premium}

As an ex-ante systemic risk measure, Huang, Zhou and Zhu (2010) proposed distress insurance as a theoretical insurance premium against systemic financial distress. Their methodology is based on each institution's marginal contribution to systemic risk. First, the probability of default is calculated as:

$$
\mathrm{PoD}_{\mathrm{i}, \mathrm{t}}=\frac{a_{\mathrm{t}} \mathrm{s}_{\mathrm{i}, \mathrm{t}}}{a_{\mathrm{t}} \mathrm{LGD}_{\mathrm{i}, \mathrm{t}}+b_{\mathrm{t}} \mathrm{s}_{\mathrm{i}, \mathrm{t}}}
$$

where $a_{\mathrm{t}} \equiv \int_{\mathrm{t}}^{\mathrm{t}+\mathrm{T}} e^{-r \tau} \mathrm{d} \tau, b_{\mathrm{t}} \equiv \int_{\mathrm{t}}^{\mathrm{t}+\mathrm{T}} \tau e^{-r \tau} \mathrm{d} \tau$, LGD is the loss given default, $r$ is the risk-free rate and $\mathrm{s}_{\mathrm{i}, \mathrm{t}}$ is the CDS spread of bank $i$ at time $t$. Next the correlation between banks' assets is estimated and then a hypothetical debt portfolio is created that consists of the liabilities of all the sampled banks weighted by the Size of the liability in each institution. The cost of the distress insurance premium is the expectation of portfolio credit losses equalling or exceeding a predetermined threshold, simulated using the Monte Carlo method in two steps: (1) the probability distribution of joint default is calculated; and (2) the LGD distribution is incorporated so as to derive the probability distribution of portfolio losses.

\subsubsection{Broader hedge fund-based systemic risk measures}

The broad impact of hedge funds on systemic risk was analysed by Chan, Getmansky, Haas and Lo (2006a, 2006b), who, to examine the unique risk/return profiles of hedge funds, suggested three risk measures. In the first measure, an autocorrelation-based measure is used to proxy hedge fund illiquidity exposure, with 
the authors adapting a cross-sectional weighted average of rolling first-order autocorrelations for hedge funds. The second measure quantifies the probability of hedge fund liquidation and the third measure is a regime-switching model that quantifies aggregate distress in the hedge fund sector.

\subsubsection{Granger causality tests}

Billio et al. (2010) developed two measures of connectedness for capturing changes in correlation and causality among financial institutions, based on an analysis of contagion in four financial sectors, namely, hedge funds, banks, brokers and insurance companies. The first measure, which determines increases in correlation between asset returns, is based on PCA, which decomposes the asset return of a sample of financial institution into orthogonal factors of decreasing explanatory power (see Muirhead, 1982 for an explanation of PCA). The second measure uses pairwise linear and nonlinear Granger causality tests to determine the network of relations between financial institutions. This test gives a statistical notion of causality based on the relative forecasting power of series of pairs. From this model, Billio et al. (2010) made the following network-based measures of connectedness: degree of Granger causality (DGC), number of connections, sector-conditional connections, closeness and eigenvector centrality. Resolved measures can identify and quantify financial crisis periods and act as early-warning indicators of systemic risk.

\subsubsection{Simulating a credit scenario}

Chau-Lau, Espinosa and Sole (2009) evaluated a systemic banking network and interbank linkage. Their analysis considered a network of $N$ institutions and stylized balance sheets for each financial institution $i$, as follow:

$$
\sum_{j} x_{j i}+a_{i}=k_{i}+b_{i}+d_{i}+\sum_{j} x_{i j}
$$

where $x_{j i}$ represents bank $i$ loans to bank $j, a_{i}$ is bank $i$ 's other assets, $k_{i}$ is bank $i$ 's capital, $b_{i}$ is long- and short-term borrowing that is not interbank lending, $x_{i j}$ represents bank $i$ borrowing from bank $j$ and $d_{i}$ is a deposit in bank $i$. This approach analyses contagion due to a credit shock between institutions and tracks, via simulation, the domino effects of each individual failure. 


\subsubsection{GDP stress test}

Alfaro and Drehmann (2009) developed a macroeconomic stress test - useful for assessing the potential impact of adverse shocks - using an absorption ratio model of actual and expected real GDP growth $\left(y_{t}\right)$ as good summary indicators of macroeconomic status. Their model is defined as:

$$
y_{t}=\rho_{1} y_{t-1}+\rho_{2} y_{t-2}+\varepsilon_{t} .
$$

As a stress scenario, the authors used the worst negative forecast error of the above absorption ratio model, regardless of whether this coincided with a banking crisis or not.

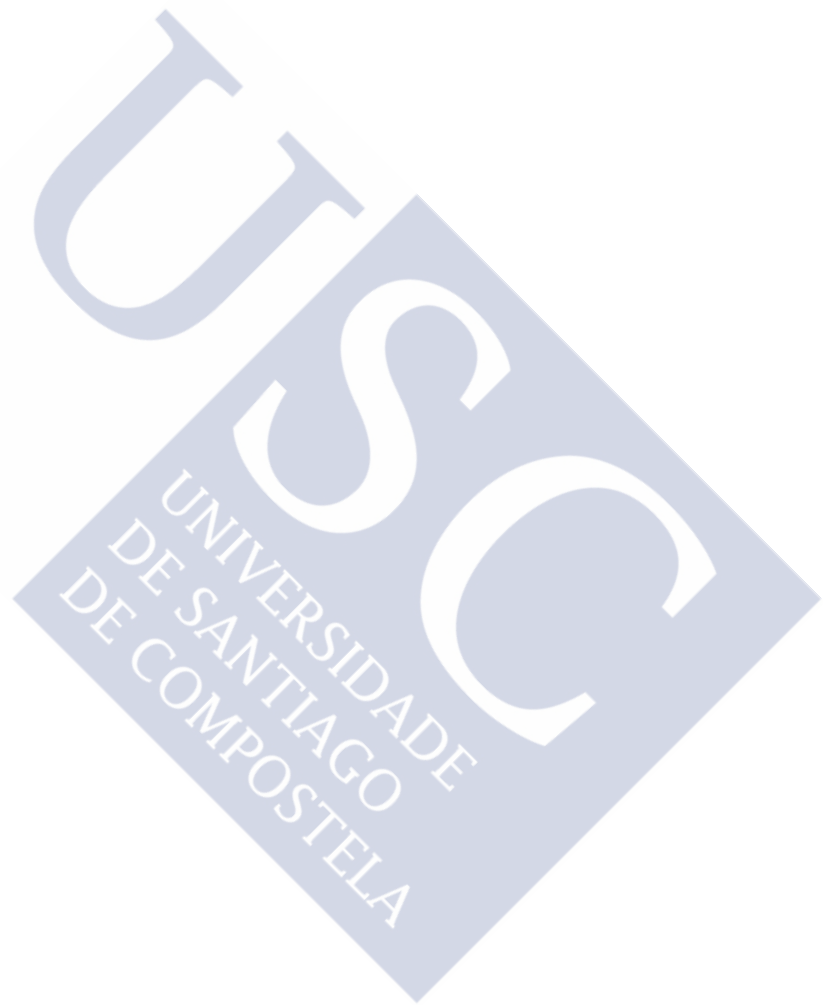




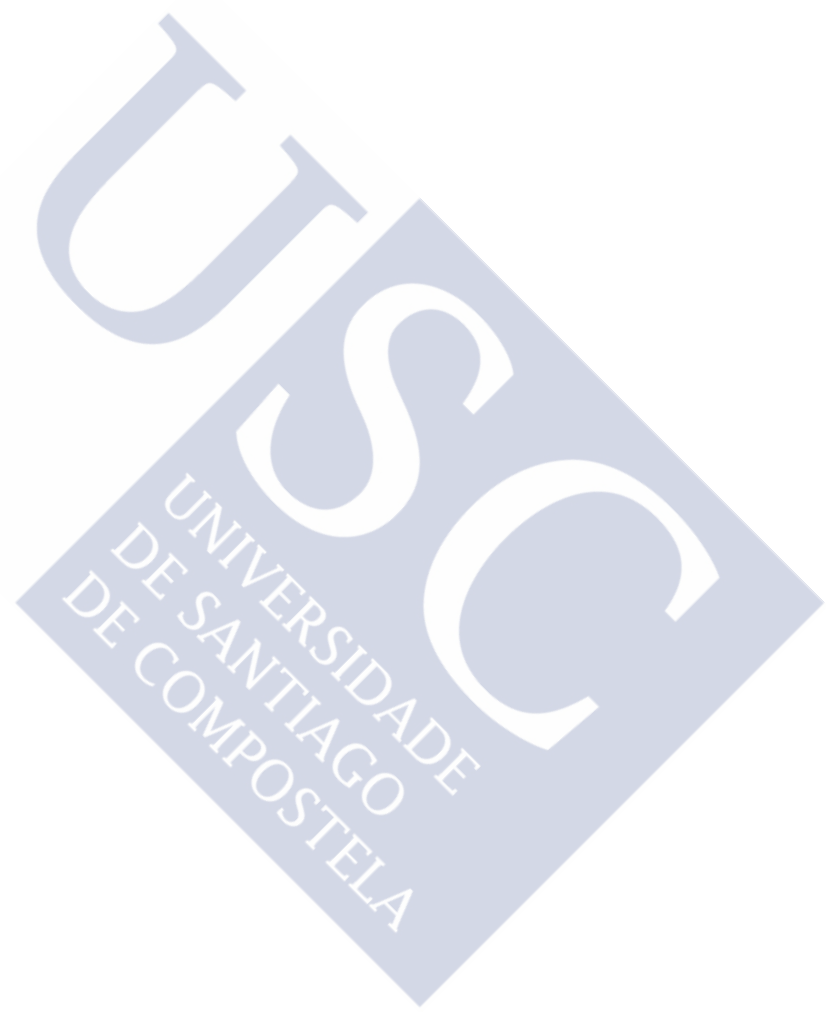




\section{Chapter 2}

\section{Measuring systemic risk in the Spanish financial system: A CoVaR approach}

\subsection{Introduction}

Financial crises draw the attention of investors and regulators to the fragility of the financial system and the potential systemic risk effects of a bank default. Quantifying systemic risk is therefore crucial to an assessment of how the fragile position of a financial institution could impair the performance of other financial institutions. It is also crucial to determine how much regulatory capital a financial institution needs to hoard in order to cover its exposure to this kind of risk. Below we quantify systemic risk for Spanish financial institutions and examine how this risk has changed in the recent global financial crisis and the European sovereign debt crisis.

The most widely used risk measure is value-at-risk (VaR), which quantifies a financial institution's maximum loss for a given confidence level and time horizon. This measure, however, is centred on the individual risk of an institution and fails to consider the potential spillover effects that default may have on other institutions. The literature has proposed a large number of systemic risk measures (see Bisias et al., 2012 and Bernal et al., 2013 for a review). Huang, Zhou and Zho (2009) developed a systemic risk indicator given by the price of credit default swaps (CDS) 
against systemic financial distress. Using CDS data, Segoviano and Goodhart (2009) constructed a banking stability index with which to assess interbank dependence for tail events. Moreno and Peña (2012) provided evidence regarding the suitability of using CDS data to estimate systemic risk. Acharya et al. (2010) introduced systemic expected shortfall and marginal expected shortfall as indicators to quantify downside risk and the contributions of financial institutions to risk. Brownless and Engle (2012) developed a systemic risk measure called SRISK, representing the amount of capital needed to restore a minimal capital requirement. Allen et al. (2010) proposed a measure of aggregate systemic risk called CATFIN that can predict declines in aggregate bank lending activity six months in advance. Billio et al. (2012) proposed five measures of systemic risk that capture contagion and exposure effects in relationships between financial institutions. Engle and Manganelli (2004) developed a conditional autoregressive value at risk (CAViaR) model that uses quantile regression to capture the tail behaviour of returns.

Recently, Adrian and Brunnermeier (2011) proposed conditional VaR $(\mathrm{CoVaR})$ as a new measure of systemic risk. CoVaR captures possible risk spillovers between financial institutions by providing information on the VaR of the financial system conditional on the fact that a financial institution is in distress, ${ }^{1}$ with the systemic risk contribution of an institution measured as the difference between the $\mathrm{CoVaR}$ under financial distress and the $\mathrm{CoVaR}$ in its benchmark state. More recently, Girardi and Ergün (2013) generalized the CoVaR measure by assuming that the conditioning financial distress event should refer to the return of the financial institution being less than or equal to its VaR, rather than merely being equal to its VaR, as proposed in Adrian and Brunnermeier (2011). Girardi and Ergün (2013) also proposed a new approach to quantifying systemic risk that differs from the quantile regression approach proposed by Adrian and Brunnermeier (2011); it consists of using a multivariate generalized autoregressive conditional heteroskedasticity (MGARCH) model to characterize joint density between the financial system and financial institution returns and to obtain the CoVaR value by numerically solving a double integral.

Below we quantify systemic risk for Spanish financial institutions using the $\mathrm{CoVaR}$ measure and also account for the quantitative effect on the CoVaR value of

\footnotetext{
${ }^{1}$ López-Espinosa et al. (2012) identified the main determinants of systemic risk for a set of large international banks using the CoVaR measure proposed by Adrian and Brunnermeier (2011).
} 
using quantile regression and MGARCH. We also propose a novel copula-based approach to computing the $\mathrm{CoVaR}$ value, given that copulas are more flexible in modelling joint distributions and are particularly useful for characterizing tail behaviour, which provides such crucial information for the CoVaR. Estimating the $\mathrm{CoVaR}$ through copulas is also computationally less cumbersome than using the MGARCH approach. The procedure involves two steps: first, given the confidence level of the $\mathrm{VaR}$ and the $\mathrm{CoVaR}$, we obtain the cumulative probability of the $\mathrm{CoVaR}$ from the copula; and second, we invert the marginal distribution function for this cumulative probability and so obtain the value of the CoVaR.

We studied systemic risk for financial institutions listed on the Spanish stock exchange using weekly data for the period January 2003 to February 2013. Our evidence shows that systemic risk displays dynamic behaviour that is well captured by the copula and MGARCH approach to computing the CoVaR; quantile regression, however, is unable to capture the dynamics and abrupt changes in the value of systemic risk. More specifically, we found significant increases in systemic risk during the recent global financial crisis and the European debt crisis that quantile regression was unable to capture or underestimated. The fact that the copula approach, on average, indicated greater systemic risk than the MGARCH approach is consistent with the time-varying evidence of tail dependence reported by the copula.

The remainder of the chapter is laid out as follows. Section 2.2 characterizes systemic risk, quantile regression, MGARCH and copula approaches to the CoVaR, Section 2.3 presents our data, Section 2.4 reports the results and Section 2.5 concludes the chapter.

\subsection{Methodology}

Several systemic risk measures have been proposed in the literature to quantify the impact of a potentially risky financial institution on the financial system as a whole. For our research, we chose to use $\mathrm{VaR}$ - as arguably the measure most widely employed by financial institutions - to quantify systemic risk as the effect of the risky situation of a particular financial institution on the $\mathrm{VaR}$ of the financial system overall, specifically, the CoVaR (Adrian and Brunnermeier, 2011; Girardi and Ergün, 2013). 


\subsubsection{CoVaR}

The CoVaR of the financial system is the VaR of the financial system conditional on the fact that a given financial institution is in financial distress. Let $X_{t}^{s}$ be the returns of the financial system and let $X_{t}^{i}$ be the returns of the financial institution $i$. The CoVaR for a confidence level $1-\beta$ and time $\mathrm{t}$ can be formally defined as the $\beta$-quantile of the conditional distribution of $X_{t}^{s}$ :

$$
\operatorname{Pr}\left(\mathrm{X}_{\mathrm{t}}^{\mathrm{s}} \leq \mathrm{CoVaR}_{t, \beta}^{\mathrm{s} \mid \mathrm{i}} \mid \mathrm{X}_{\mathrm{t}}^{\mathrm{i}} \leq \operatorname{VaR}_{\mathrm{t}, \alpha}^{\mathrm{i}}\right)=\beta,
$$

where the financial distress situation of the financial institution $i$ is represented by the fact that $\mathrm{X}_{\mathrm{t}}^{\mathrm{i}} \leq \mathrm{VaR}_{\mathrm{t}, \alpha}^{\mathrm{i}}$, where $\operatorname{VaR}_{\mathrm{t}, \alpha}^{\mathrm{i}}$ is the $\mathrm{VaR}$ for the financial institution $i$, measuring the maximum loss that financial institution $i$ may experience for a confidence level $1-\alpha$ and a specific time horizon $t$, that is, the $\alpha$-quantile of the return distribution for the financial institution $i: \operatorname{Pr}\left(\mathrm{X}_{\mathrm{t}}^{\mathrm{i}} \leq \operatorname{VaR}_{\alpha, \mathrm{t}}^{\mathrm{i}}\right)=\alpha$. Thus, from a statistical point of view, computing the $\mathrm{CoVaR}$ value consists of determining the quantile of a conditional distribution.

In addition, the systemic risk contribution of a particular financial institution $i$ can be defined as the difference between the CoVaR for a confidence level $1-\beta$ and the $\mathrm{VaR}$ of the financial system conditional on the fact that financial institution $i$ is in a benchmark state, measured as the median of the return distribution of institution $i$ (the $\mathrm{VaR}$ value for $\alpha=0.5$ ). This measure, called delta CoVaR ( $\Delta$ CoVaR), is formally defined as:

$$
\Delta \mathrm{CoVaR}_{\mathrm{t}}^{\mathrm{s} \mid \mathrm{i}}=\left(\mathrm{CoVaR}_{\beta, \mathrm{t}}^{\mathrm{s} \mid \mathrm{i}}-\mathrm{CoVaR}_{\beta, \mathrm{t}}^{\mathrm{si}} \mid \mathrm{i}=0.5\right) / \operatorname{CoVaR}_{\beta, \mathrm{t}}^{\mathrm{s} \mid \mathrm{i}, \alpha=0.5}
$$

Below we describe different approaches to computing the value of the CoVaR.

\subsubsection{Quantile regression}

Adrian and Brunnermeier (2011) proposed using quantile regression to compute the $\mathrm{CoVaR}$ in such a way that the VaR of the financial institution $i$ and a set of explanatory variables determine the quantile of the conditional distribution of $\mathbf{X}_{t}^{\mathrm{s}}$. We can thus obtain information on the VaR of the financial system conditional on the fact that the returns of financial institution $i$ are in its $\operatorname{VaR}, \mathrm{X}_{\mathrm{t}}^{\mathrm{i}}=\mathrm{VaR}_{\mathrm{t}, \alpha}^{\mathrm{i}}$, that is, $\operatorname{Pr}\left(\mathrm{X}_{\mathrm{t}}^{\mathrm{s}} \leq \mathrm{CoVaR}_{t, \beta}^{\mathrm{s} \mid \mathrm{i}} \mid \mathrm{X}_{\mathrm{t}}^{\mathrm{i}}=\operatorname{VaR}_{\mathrm{t}, \alpha}^{\mathrm{i}}\right)$. We do this by characterizing the $\beta^{\text {th }}$ conditional quantile function of $\mathrm{X}_{\mathrm{t}}^{\mathrm{s}}$ as: 


$$
\mathrm{Q}_{\mathrm{X}^{\mathrm{s}}}(\beta \mid \mathrm{x})=\inf \left\{\mathrm{b} \mid \mathrm{F}_{\mathrm{x}^{\mathrm{s}}}(\mathrm{b} \mid \mathrm{x}) \geq \beta\right\}=\alpha+\beta^{\mathrm{si}} \mathrm{VaR}_{\mathrm{t}, \alpha}^{\mathrm{i}}+\gamma \mathrm{M}_{\mathrm{t}-1},
$$

where $\mathrm{F}_{\mathrm{x}^{\mathrm{s}}}(\mathrm{b} \mid \mathrm{x})$ is the conditional distribution function of $\mathrm{X}_{\mathrm{t}}^{\mathrm{s}}$ given the set $\mathrm{x}$ of explanatory variables, $\mathbf{M}_{\mathbf{t}-1}$ includes a set of explanatory variables and the quantile regression coefficient $\beta^{\text {si }}$ determines the dependence relationship between the $\operatorname{VaR}_{t, \alpha}^{\mathrm{i}}$ and the $\mathrm{VaR}$ of the financial system. The CoVaR value at each time $\mathrm{t}$ is computed as the estimated value of the quantile regression given by Eq. (2.3) for the corresponding confidence levels of $1-\beta$ and $1-\alpha \cdot{ }^{2}$ Quantile regression is computationally simple but has the disadvantage that it provides information only on the CoVaR conditional on the fact that $\mathrm{X}_{\mathrm{t}}^{\mathrm{i}}=\mathrm{VaR}_{\mathrm{t}, \alpha}^{\mathrm{i}}$ and not on the fact that $\mathrm{X}_{\mathrm{t}}^{\mathrm{i}} \leq \mathrm{VaR}_{\mathrm{t}, \alpha}^{\mathrm{i}}$; this fact has repercussions for the CoVaR values, as was discussed in Girardi and Ergün (2013). In addition, using quantile regression requires computation of the $\mathrm{VaR}_{\mathrm{t}, \alpha}^{\mathrm{i}}$

Adrian and Brunnermeier (2011) proposed computing the $\mathrm{VaR}_{\mathrm{t}, \alpha}^{\mathrm{i}}$ in Eq. (2.3) by means of quantile regression for the distribution of $\mathrm{X}_{\mathrm{t}}^{\mathrm{i}}$, where only the explanatory variables in $\mathbf{M}_{\mathrm{t}-1}$ were included in order to allow the value of $\mathrm{VaR}_{\mathrm{t}, \alpha}^{\mathrm{i}}$ to change over time. With the aim of accounting for the effect of heteroskedasticity and fat tails of the return distribution on the value of the $\operatorname{VaR}_{\mathrm{t}, \alpha}^{\mathrm{i}}$, we followed a different approach to computing the $\operatorname{VaR}_{t, \alpha}^{\mathrm{i}}$. Specifically, we modelled the returns of each financial institution through an autoregressive (AR) moving average (MA) model, specifically, $\operatorname{ARMA}(p, q)$, specified as:

$$
\mathrm{X}_{\mathrm{t}}^{\mathrm{i}}=\phi_{0}+\sum_{\mathrm{j}=1}^{\mathrm{p}} \phi_{\mathrm{j}} \mathrm{X}_{\mathrm{t}-\mathrm{j}}^{\mathrm{i}}+\sum_{\mathrm{h}=1}^{\mathrm{q}} \varphi_{\mathrm{h}} \varepsilon_{\mathrm{t}-\mathrm{h}}^{\mathrm{i}}+\varepsilon_{\mathrm{t}}^{\mathrm{i}},
$$

where $\mathrm{p}$ and $\mathrm{q}$ are non-negative integers and $\phi_{\mathrm{j}}$ and $\varphi_{\mathrm{h}}$ are the $\mathrm{AR}$ and MA parameters, respectively. The stochastic process $\varepsilon_{\mathrm{t}}^{\mathrm{i}}=\sigma_{\mathrm{i}, \mathrm{t}} \mathrm{z}_{\mathrm{i}, \mathrm{t}}$, where $\sigma_{\mathrm{i}, \mathrm{t}}^{2}$ - the conditional variance of $\varepsilon_{\mathrm{t}}^{\mathrm{i}}$ whose dynamic is reflected in a threshold generalized autoregressive conditional heteroskedasticity (TGARCH) specification (Zakoian, 1994; Glosten et al., 1993) — is given by:

\footnotetext{
${ }^{2}$ Adrian and Brunnermeier (2011) proposed a specification of the quantile regression function that is slightly different from Eq. (2.3). As the explanatory variable they include $X_{t}^{i}$ instead of $\operatorname{VaR}_{t, \alpha}^{\mathrm{i}}$ and use the estimated parameter values to predict the CoVaR by substituting $\mathrm{X}_{\mathrm{t}}^{\mathrm{i}}$ by $\operatorname{VaR}_{t, \alpha}^{i}$. In this way, they assume that estimated parameter values are equal across different quantile regression functions.
} 


$$
\sigma_{\mathrm{i}, \mathrm{t}}^{2}=\omega+\sum_{\mathrm{h}=1}^{\mathrm{d}} \beta_{\mathrm{h}} \sigma_{\mathrm{i}, \mathrm{t}-\mathrm{h}}^{2}+\sum_{\mathrm{k}=1}^{\mathrm{m}} \alpha_{\mathrm{k}}\left(\varepsilon_{\mathrm{t}-\mathrm{k}}^{\mathrm{i}}\right)^{2}+\sum_{\mathrm{r}=1}^{\mathrm{m}} \gamma_{\mathrm{r}} \varepsilon_{\mathrm{t}-\mathrm{r}}^{\mathrm{i}}
$$

where $\omega$ is a constant, $\sigma_{\mathrm{i}, \mathrm{t}-1}^{2}$ is the variance prediction error for the previous period (the generalized autoregressive conditional heteroskedasticity (GARCH) component), $\varepsilon_{t-r}^{\mathrm{i}}$ represents the volatility shock for the previous period (the autoregressive conditional heteroskedasticity $(\mathrm{ARCH})$ component) and $\gamma_{\mathrm{r}}$ captures leverage effects. When $\gamma_{\mathrm{r}}$ takes values greater than zero the future conditional variance will increase proportionally more after a negative shock that after a positive shock of the same magnitude. $\mathbf{z}_{\mathrm{i}, \mathrm{t}}$ is an i.i.d. random variable with mean zero and unit variance that follows a Hansen (1994) skewed-t density distribution given by:

$$
f\left(z_{i, t} ; v, \eta\right)=\left\{\begin{array}{ll}
b c\left(1+\frac{1}{v-2}\left(\frac{b z_{i, t}+a}{1-\eta}\right)^{2}\right)^{-(v+1) / 2} & z_{i, t}<-a / b \\
b c\left(1+\frac{1}{v-2}\left(\frac{b z_{i, t}+a}{1+\eta}\right)^{2}\right)^{-(v+1) / 2} & z_{i, t} \geq-a / b
\end{array},\right.
$$

where $v$ and $\eta$ are the degrees of freedom parameter $(2<v<\infty)$ and the symmetric parameter $(-1<\eta<1)$, respectively. The constants $\mathrm{a}, \mathrm{b}$ and $\mathrm{c}$ are given by $\mathrm{a}=4 \eta \mathrm{c}\left(\frac{\mathrm{v}-2}{\mathrm{v}-1}\right), \mathrm{b}^{2}=1+3 \eta^{2}-\mathrm{a}^{2}$ and $\mathrm{c}=\Gamma\left(\frac{\mathrm{v}+1}{2}\right) / \sqrt{\pi(\mathrm{v}-2)} \Gamma\left(\frac{\mathrm{v}}{2}\right)$. This distribution converges to the standard Gaussian as $\eta=0$ and $v \rightarrow \infty$ and to the symmetric Student-t distribution as $\eta=0$ and $v$ is finite. From Eqs. (2.4)-(2.6) we can compute the $\operatorname{VaR}_{\mathrm{t}, \alpha}^{\mathrm{i}}$ at each time $\mathrm{t}$ as:

$$
\mathrm{VaR}_{\mathrm{t}, \alpha}^{\mathrm{i}}=\phi_{0}+\sum_{\mathrm{j}=1}^{\mathrm{p}} \phi_{\mathrm{j}} \mathrm{X}_{\mathrm{t}-\mathrm{j}}^{\mathrm{i}}+\sum_{\mathrm{h}=1}^{\mathrm{p}} \varphi_{\mathrm{h}} \varepsilon_{\mathrm{t}-\mathrm{h}}^{\mathrm{i}}+\sigma_{\mathrm{i}, \mathrm{t}}^{2} \mathrm{z}_{\mathrm{i}, \mathrm{t}}^{-1}(\alpha),
$$

where $z_{i, t}^{-1}(\alpha)$ is the quantile of the standardized skewed-t distribution for probability $\alpha$. This is the value for the VaR that we considered in Eq. (2.3) in order to estimate the CoVaR through quantile regression.

\subsubsection{MGARCH}

In order to obtain the value of the $\mathrm{VaR}$ conditional on the event $\mathrm{X}_{\mathrm{t}}^{\mathrm{i}} \leq \mathrm{VaR}_{\mathrm{t}, \alpha}^{\mathrm{i}}$, Girardi and Ergün (2013) proposed an alternative procedure based on the joint distribution of $\mathbf{X}_{\mathbf{t}}^{\mathrm{s}}$ and $\mathbf{X}_{\mathrm{t}}^{\mathrm{i}}$. From Eq. (2.1), the CoVaR can be expressed as: 


$$
\frac{\operatorname{Pr}\left(\mathrm{X}_{\mathrm{t}}^{\mathrm{s}} \leq \mathrm{CoVaR}_{t, \beta}^{\mathrm{s} \mid \mathrm{i}}, \mathrm{X}_{\mathrm{t}}^{\mathrm{i}} \leq \mathrm{VaR}_{\mathrm{t}, \alpha}^{\mathrm{i}}\right)}{\operatorname{Pr}\left(\mathrm{X}_{\mathrm{t}}^{\mathrm{i}} \leq \mathrm{VaR}_{\mathrm{t}, \alpha}^{\mathrm{i}}\right)}=\beta
$$

Given that $\operatorname{Pr}\left(\mathrm{X}_{\mathrm{t}}^{\mathrm{i}} \leq \operatorname{VaR}_{\mathrm{t}, \alpha}^{\mathrm{i}}\right)=\alpha$, Eq. (2.8) can be written as:

$$
\operatorname{Pr}\left(\mathrm{X}_{\mathrm{t}}^{\mathrm{s}} \leq \mathrm{CoVaR}_{t, \beta}^{\mathrm{s} \mid \mathrm{i}}, \mathrm{X}_{\mathrm{t}}^{\mathrm{i}} \leq \operatorname{VaR}_{\mathrm{t}, \alpha}^{\mathrm{i}}\right)=\alpha \beta
$$

Hence, the value of the CoVaR can be obtained by numerically solving the following double integral:

$$
\int_{-\infty}^{C o V a R_{t, \beta}^{s i \mathrm{i}}} \int_{-\infty}^{V a R_{t, \alpha}^{\mathrm{i}}} \operatorname{pdf}_{\mathrm{t}}\left(\mathrm{X}_{\mathrm{t}}^{\mathrm{s}}, \mathrm{X}_{\mathrm{t}}^{\mathrm{i}}\right) \mathrm{d} \mathrm{X}_{\mathrm{t}}^{\mathrm{s}} \mathrm{dX} \mathrm{X}_{\mathrm{t}}^{\mathrm{i}}=\alpha \beta
$$

for $\operatorname{CoVaR}_{t, \beta}^{\mathrm{s} \mid \mathrm{i}}$, where $\operatorname{pdf}_{\mathrm{t}}\left(\mathrm{X}_{\mathrm{t}}^{\mathrm{s}}, \mathrm{X}_{\mathrm{t}}^{\mathrm{i}}\right)$ denotes the joint probability density. Thus, computing the CoVaR involves knowledge of the joint distribution of $\mathbf{X}_{\mathbf{t}}^{\mathrm{s}}$ and $\mathbf{X}_{\mathbf{t}}^{\mathrm{i}}$.

According to Girardi and Ergün (2013), the joint distribution of $\mathbf{X}_{\mathrm{t}}^{\mathrm{s}}$ and $\mathbf{X}_{\mathrm{t}}^{\mathrm{i}}$ can be obtained using MGARCH and time-yarying dynamic conditional correlation (DCC). This MGARCH-DCC model, initially proposed by Engle (2002), considers the mean and volatility dynamics of the returns as given by Eqs. (2.4) and (2.5). The bivariate stochastic terms are given by $\varepsilon_{\mathrm{t}}=\Sigma_{\mathrm{t}}^{1 / 2} \mathrm{Z}_{\mathrm{t}}$, where $\varepsilon_{\mathrm{t}}=\left(\varepsilon_{\mathrm{t}}^{\mathrm{s}} \varepsilon_{\mathrm{t}}^{\mathrm{s}}\right)^{\prime}$, $\mathrm{z}_{\mathrm{t}}=\left(\mathrm{z}_{\mathrm{s}, \mathrm{t}} \mathrm{z}_{\mathrm{i}, \mathrm{t}}\right)^{\prime}$ has a standardized bivariate Student-t distribution and where $\Sigma_{\mathrm{t}}$ is a variance-covariance matrix where the variance of each stochastic component is given by Eq. (2.5) and the covariance between the two stochastic components is given by $\sigma_{\mathrm{si}, \mathrm{t}}=\rho_{\mathrm{si}, \mathrm{t}} \sqrt{\sigma_{\mathrm{s}, \mathrm{t}}^{2} \sigma_{\mathrm{i}, \mathrm{t}}^{2}}$, where $\rho_{\mathrm{si}, \mathrm{t}}$ is the correlation coefficient between the returns of the system and the financial institution $i$. The correlation matrix is given by $\mathrm{R}_{\mathrm{t}}=\mathrm{D}_{\mathrm{t}}^{-1 / 2} \Sigma_{\mathrm{t}} \mathrm{D}_{\mathrm{t}}^{-1 / 2}$, where $\mathrm{D}_{\mathrm{t}}$ is a $2 \mathrm{x} 2$ diagonal matrix, with the conditional variance of each variable located along the main diagonal. Engle (2002) proposed characterizing the dynamics of the conditional correlations as follows:

$$
\begin{gathered}
\mathrm{R}_{\mathrm{t}}=\operatorname{diag}\left(\mathrm{Q}_{\mathrm{t}}\right)^{-1 / 2} \mathrm{Q}_{\mathrm{t}} \operatorname{diag}\left(\mathrm{Q}_{\mathrm{t}}\right)^{-1 / 2} \\
\mathrm{Q}_{\mathrm{t}}=(1-\alpha-\beta) \overline{\mathrm{Q}}+\alpha\left(\varepsilon_{\mathrm{t}-1} \varepsilon_{\mathrm{t}-1}^{\prime}\right)+\beta \mathrm{Q}_{\mathrm{t}-1}
\end{gathered}
$$

where $\overline{\mathrm{Q}}$ is the unconditional covariance matrix for the standardized residuals, $\operatorname{diag}\left(\mathrm{Q}_{\mathrm{t}}\right)$ is the $\mathrm{Q}_{\mathrm{t}}$ diagonal matrix and $\alpha$ and $\beta$ are parameters. Once we estimated the MGARCH-DCC model, we had all the necessary information on the elliptical $\operatorname{pdf}_{\mathrm{t}}\left(\mathrm{X}_{\mathrm{t}}^{\mathrm{s}}, \mathrm{X}_{\mathrm{t}}^{\mathrm{i}}\right)$, so, for the given value of the $\operatorname{VaR}_{\mathrm{t}, \alpha}^{\mathrm{i}}$ we could numerically solve Eq. (10) to obtain the CoVaR for each time period. 


\subsubsection{Copulas}

We can employ copulas ${ }^{3}$ to compute the CoVaR. From Eq. (2.9), we can express the CoVaR in terms of the joint distribution function of $X_{t}^{s}$ and $X_{t}^{i}, F_{X_{t}^{s}, X_{t}^{i}}$, as:

$$
\mathrm{F}_{\mathrm{X}_{\mathrm{t}}^{\mathrm{s}}, \mathrm{X}_{\mathrm{t}}^{\mathrm{i}}}\left(\mathrm{CoVaR}_{\mathrm{t}, \beta}^{\mathrm{s} \mid \mathrm{i}}, \operatorname{VaR}_{\mathrm{t}, \alpha}^{\mathrm{i}}\right)=\alpha \beta .
$$

Furthermore, according to Sklar's (1959) theorem, the joint distribution function of two continuous random variables can be expressed in terms of a copula function. Hence, Eq. (2.12) can be written as:

$$
\mathrm{C}\left(\mathrm{F}_{\mathrm{X}_{\mathrm{t}}^{\mathrm{s}}}\left(\mathrm{CoVaR}_{\mathrm{t}, \beta}^{\mathrm{s} \mid \mathrm{i}}\right), \mathrm{F}_{\mathrm{X}_{\mathrm{t}}^{\mathrm{i}}}\left(\operatorname{VaR}_{\mathrm{t}, \alpha}^{\mathrm{i}}\right)\right)=\alpha \beta
$$

where $\mathrm{C}(\cdot, \cdot)$ is a copula function and $\mathrm{F}_{\mathbf{X}_{\mathrm{t}}^{s}}$ and $\mathrm{F}_{\mathrm{X}_{\mathrm{t}}^{\mathrm{i}}}$ denote the marginal distributions of $X_{t}^{s}$ and $X_{t}^{i}$, respectively, such that $u=F_{X_{t}^{s}}\left(\operatorname{CoVaR}_{t, \beta}^{s \mid i}\right)$ and $v=F_{X_{t}^{i}}\left(\operatorname{VaR}_{t, \alpha}^{i}\right)$. Thus, from Eq. (2.13) we can compute the CoVaR value following a two-step procedure. First, given that $\mathrm{v}=\alpha$ and given the copula specification, for a confidence level $1-\beta$ we compute the cumulative probability for the CoVaR, $\mathrm{u}$, by solving from Eq. (2.13). Next, from $\mathrm{u}$, we invert the marginal distribution function of $\mathrm{X}_{\mathrm{t}}^{\mathrm{s}}$ to obtain the CoVaR, hence, $\mathrm{CoVaR}_{\mathrm{t}, \beta}^{\mathrm{s} i \mathrm{i}}=\mathrm{F}_{\mathrm{X}_{\mathrm{t}}^{\mathrm{s}}}^{-1}(\mathrm{u})$.

Computing the CoVaR using copulas offers three main advantages. First, copulas offer more flexibility in separate modelling the marginals and dependence, which is especially important when the marginals have different characteristics or when dependence is not linear - in particular, when the joint distribution displays different forms of tail dependence (crucial for the values of the CoVaR). Second, computation of the CoVaR through copulas is less computationally burdensome than using the MGARCH approach involving the numerical resolution of a double integral. Third, computation of the $\mathrm{VaR}_{\mathrm{t}, \alpha}^{\mathrm{i}}$ is not necessary, as we only need information on the confidence level for $\mathrm{VaR}_{\mathrm{t}, \alpha}^{\mathrm{i}}$, which is exogenously determined.

Obtaining the CoVaR through copulas requires specification of the marginals and the copula function. The marginals we used are given by Eqs. (2.4)-(2.6); in order to characterize different patterns of dependence, different copula specifications,

\footnotetext{
${ }^{3}$ For further analysis of copulas, see Joe (1997) and Nelsen (2006). An overview of copula applications to finance can be found in Cherubini et al. (2004). Mainik and Schaanning (2012) provided the first representation of the CoVaR in terms of copulas.
} 
as reported in Table 2.1, were used. We also captured time-varying dependence by assuming that copula parameters change over time. For the Gaussian and the Student-t copulas, we adopted an $\operatorname{ARMA}(1, \mathrm{q})$-type process (Patton, 2006) for the linear dependence parameter $\rho_{\mathrm{t}}$ :

$$
\rho_{\mathrm{t}}=\Lambda\left(\psi_{0}+\psi_{1} \rho_{\mathrm{t}-1}+\psi_{2} \frac{1}{\mathrm{q}} \sum_{\mathrm{j}=1}^{\mathrm{q}} \Phi^{-1}\left(\mathrm{u}_{\mathrm{t}-\mathrm{i}}\right) \cdot \Phi^{-1}\left(\mathrm{v}_{\mathrm{t}-\mathrm{i}}\right)\right),
$$

where $\Lambda(\mathrm{x})=\left(1-\mathrm{e}^{-\mathrm{x}}\right)\left(1+\mathrm{e}^{-\mathrm{x}}\right)^{-1}$ is the modified logistic transformation that keeps the value of $\rho_{\mathrm{t}}$ in $(-1,1)$. For the Student-t copula, $\Phi^{-1}(\mathrm{x})$ is replaced by $\mathrm{t}_{\mathrm{v}}^{-1}(\mathrm{x})$. We also considered time-varying dependence for the Gumbel copula and for its rotated version by assuming that the parameters follow the dynamics given by the following equation:

$$
\delta_{\mathrm{t}}=\bar{\omega}+\bar{\beta} \delta_{\mathrm{t}-1}+\bar{\alpha} \frac{1}{\mathrm{q}} \sum_{\mathrm{j}=1}^{\mathrm{q}}\left|\mathrm{u}_{\mathrm{t}-\mathrm{i}}-\mathrm{v}_{\mathrm{t}-\mathrm{i}}\right| .
$$

Overall, the family of eleven copulas considered here can be classified as follows: (1) symmetric copulas, with either tail dependence (Student-t and time-varying Student-t copulas) or tail independence (Gaussian, time-varying Gaussian and Plackett copulas) and (2) asymmetric tail dependence copulas (Gumbel, rotated Gumbel, BB7, BB1 and time-varying Gumbel and rotated Gumbel copulas).

The parameters of the marginal and copula models were estimated using a twostep procedure called inference functions for margins (Joe and $\mathrm{Xu}, 1996$ ). We first estimated the marginal models by maximum likelihood (ML) and then transformed each filtered standardized return series (standardized residual) into its uniform marginal via the probability integral transform, thus obtaining $\hat{u}_{t}$ and $\hat{v}_{t}$. Using this information, we then estimated the copula function parameters by ML. The number of lags in the mean and variance equations for each series was selected according to the Bayesian information criterion (BIC) and Akaike information criterion (AIC). The performance of the different copula models was evaluated using the AIC adjusted for small-sample bias, as in Breymann et al. (2003) and Reboredo (2011; 2013). 
Table 2.1: Copula specifications

\begin{tabular}{|l|l|l|l|}
\hline Name & Copula & Parameter & Structure dependence \\
\hline Gaussian & $\mathrm{C}_{\mathrm{N}}(\mathrm{u}, \mathrm{v} ; \rho)=\Phi\left(\Phi^{-1}(\mathrm{u}), \Phi^{-1}(\mathrm{v})\right)$ & & No tail dependence. \\
\hline Student-t & $\mathrm{C}_{\mathrm{ST}}(\mathrm{u}, \mathrm{v} ; \rho, \mathrm{v})=\mathrm{T}\left(\mathrm{t}-1(\mathrm{u}), \mathrm{t}_{\mathrm{v}}^{-1}(\mathrm{v})\right)$ & & Symmetric tail dependence. \\
\hline Gumbel & $\mathrm{C}_{\mathrm{G}}(\mathrm{u}, \mathrm{v} ; \delta)=\exp \left(-\left((-\log \mathrm{u})^{\delta}+(-\log \mathrm{v})^{\delta}\right)^{1 / \delta}\right)$ & $\delta \geq 1$ & $\begin{array}{l}\text { Upper tail dependence and lower tail } \\
\text { independence. When } \delta=1 \text { two variables are } \\
\text { independent. }\end{array}$ \\
\hline Rotated Gumbel & $\mathrm{C}_{\mathrm{RG}}(\mathrm{u}, \mathrm{v} ; \delta)=\mathrm{u}+\mathrm{v}-1+\mathrm{C}_{\mathrm{G}}(1-\mathrm{u}, 1-\mathrm{v} ; \delta)$ & $\delta \geq 1$ & $\begin{array}{l}\text { Upper tail independence and lower tail } \\
\text { dependence. }\end{array}$ \\
\hline BB7 & $\mathrm{C}_{\mathrm{BB} 7}(\mathrm{u}, \mathrm{v} ; \delta, \theta)=1-\left(1-\left[\left(1-(1-\mathrm{u})^{\theta}\right)^{-\delta}+\left(1-(1-\mathrm{v})^{\theta}\right)^{-\delta}-1\right]^{-1 / \delta}\right)^{1 / \theta}$ & $\begin{array}{l}\theta \geq 1, \\
\delta>0\end{array}$ & $\begin{array}{l}\text { Differing degrees of upper and lower tail } \\
\text { dependence. }\end{array}$ \\
\hline Plackett & $\mathrm{C}_{\mathrm{P}}(\mathrm{u}, \mathrm{v} ; \theta)=\frac{1}{2(\theta-1)}(1+(\theta-1)(\mathrm{u}+\mathrm{v}))-\sqrt{(1+(\theta-1)(\mathrm{u}+\mathrm{v}))^{2}-4 \theta(\theta-1) \mathrm{uv}}$ & $\begin{array}{l}\theta>0 \\
\theta \neq 1\end{array}$ & Symmetric tail independence. \\
\hline BB1 & $\mathrm{C}_{\mathrm{CG}}(\mathrm{u}, \mathrm{v} ; \delta, \theta)=\left\{\left[(\mathrm{u}-\theta-1)^{\delta}+\left(\mathrm{v}^{-\theta}-1\right)^{\delta}\right]^{1 / \delta}+1\right\}$ & $\begin{array}{l}\theta>0 \\
\delta \geq 1\end{array}$ & Asymmetric tail dependence. \\
\hline
\end{tabular}




\subsection{Data}

We empirically examined systemic risk for nine listed Spanish institutions using daily prices for the period 16 January 2003 to 28 February 2013. The set included seven banks (BBVA, Banco Santander, Banco Sabadell, Banco Popular, Bankinter, Banesto and Banco de Valencia) and two insurance companies (Catalana Occidente and Mapfre). To capture the behaviour of the whole financial system, we used the MSCI Spain Financials Index. Data were obtained from Bloomberg and returns for price data were computed on a continuous compounding basis.

We also considered daily information for a set of variables that were included in $\mathrm{M}_{\mathrm{t}-1}$ in Eq. (2.3), as follows (all these data were obtained from Bloomberg):

(a) Daily IBEX-35 volatility, computed as the standard deviation of the daily returns in a backward window of three months.

(b) The first difference of the 12-month Treasury bill rate.

(c) The slope of the yield curve measured as the difference between the 10-year and the 12-month Treasury bill rates.

(d) The credit spread determined as the difference between interest rates for corporate and government 10-year maturity bonds.

(e) Daily market returns obtained from the IBEX-35 general market index.

Figure 2.1 shows the time-series plot for the returns of the studied institutions $\left(\mathrm{X}_{\mathrm{t}}^{\mathrm{i}}\right)$ and the MSCI $\left(\mathrm{X}_{\mathrm{t}}^{\mathrm{s}}\right)$. All series display the usual characteristics of financial returns - including volatility clustering and fat tails - and also show abrupt changes around the onset of the global financial crisis (mid-2008) and European sovereign debt crisis (at the end of 2009). Figure 2.2 depicts the temporal dynamics of the set of variables considered as explanatory variables in the quantile regression. 
Chapter 2

Figure 2.1: Time series plot of daily returns for each institution and the MSCI Index.
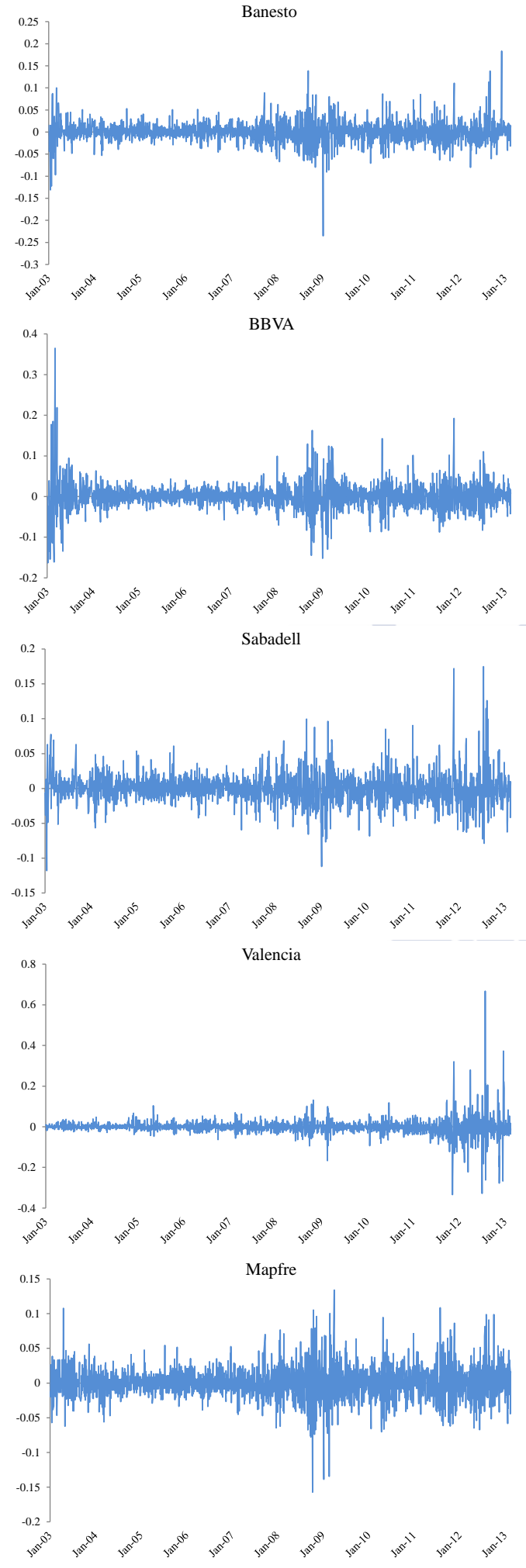
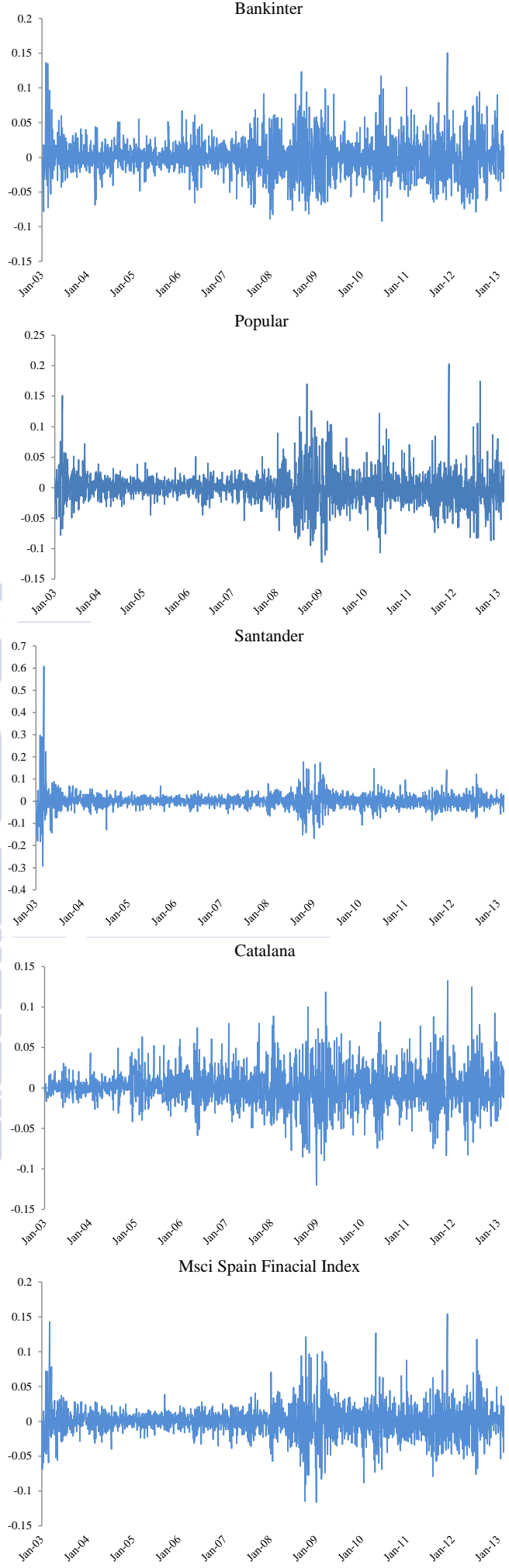

26 
Figure 2.2: Time series plot for the explanatory variables.

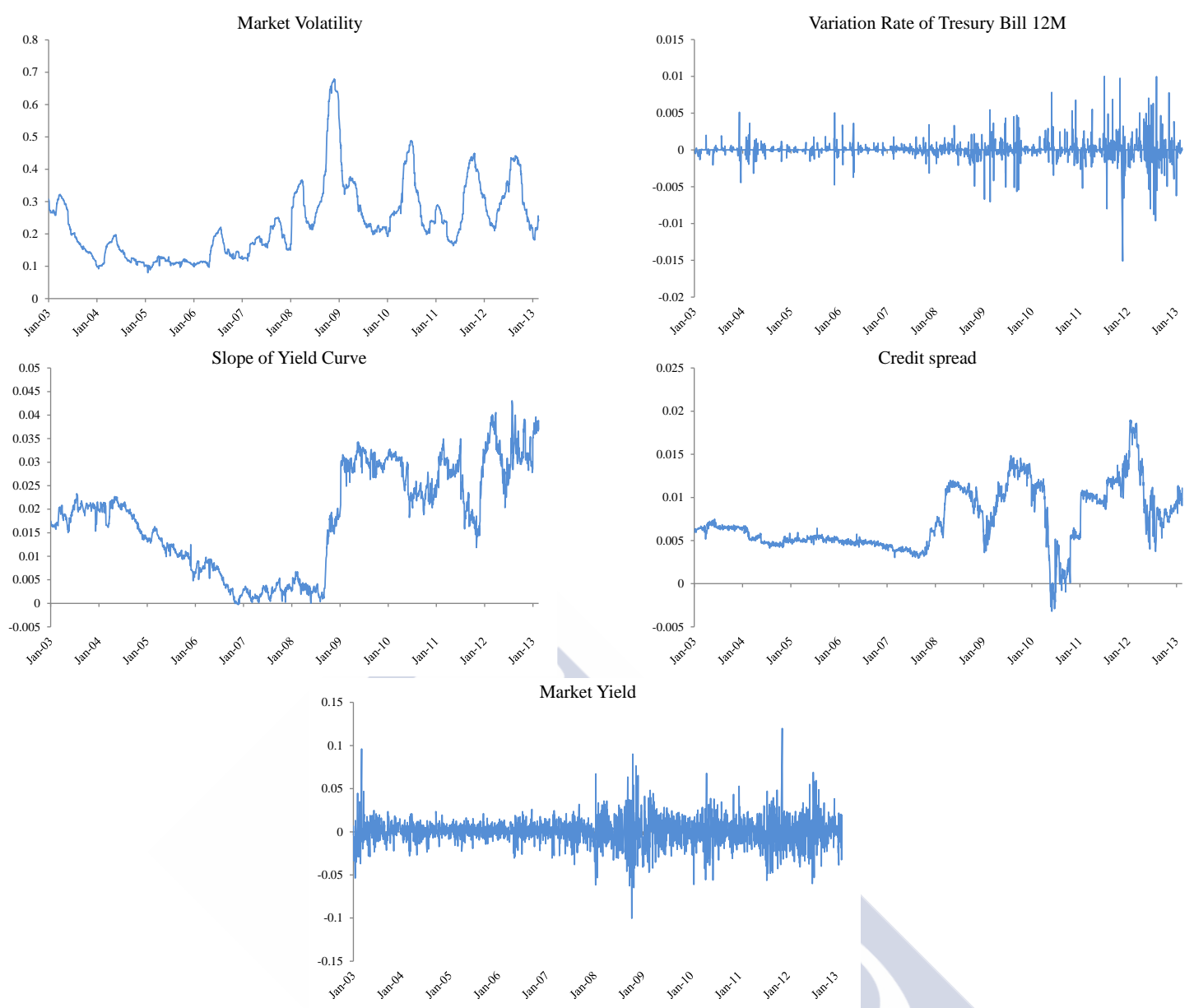

Table 2.2: Descriptive statistics for specific risk factors.

\begin{tabular}{lccccc}
\hline & $\begin{array}{c}\text { Market } \\
\text { volatility }\end{array}$ & $\begin{array}{c}\text { 12-month Treasury } \\
\text { bill variation rate }\end{array}$ & Slope & $\begin{array}{c}\text { Credit } \\
\text { spread }\end{array}$ & $\begin{array}{c}\text { Market } \\
\text { yield }\end{array}$ \\
\hline Mean & 0.232 & 0.000 & 0.018 & 0.007 & 0.000 \\
Std. Dev. & 0.113 & 0.001 & 0.011 & 0.004 & 0.015 \\
Maximum & 0.679 & 0.010 & 0.043 & 0.019 & 0.120 \\
Minimum & 0.080 & -0.015 & 0.000 & -0.003 & -0.100 \\
Skewness & 1.249 & -0.460 & -0.073 & 0.572 & 0.161 \\
Kurtosis & 4.909 & 38.921 & 1.788 & 3.209 & 8.687 \\
J-B & $987.6^{*}$ & $129011.4^{*}$ & $148.9^{*}$ & $135.1^{*}$ & $3242.4^{*}$ \\
Q(20) & 44079 & 97.319 & 45005 & 41735 & 61.509 \\
& {$[0.000]$} & {$[0.000]$} & {$[0.000]$} & {$[0.000]$} & {$[0.000]$} \\
ARCH & 33075.33 & 8.408 & 2060.849 & 1698.055 & 18.624 \\
& {$[0.000]$} & {$[0.000]$} & {$[0.000]$} & {$[0.000]$} & {$[0.000]$} \\
\hline
\end{tabular}

Note.Daily data for the period 16 January 2003 to 28 Febrary 2013. J-B denotes the Jarque-Bera statistic for normality. $\mathrm{Q}(k)$ is the Ljung-Box statistic for serial correlation in squared returns computed with $k$ lags. ARCH denotes Engle's LM test for heteroskedasticity computed using 20 lags. An asterisk (*) indicates rejection of the null hypothesis at $5 \%$. 
Table 2.3: Descriptive statistics for weekly price returns for selected Spanish financial institutions.

\begin{tabular}{lcccccccccc}
\hline & Banesto & Bankinter & BBVA & Popular & Sabadell & Santander & Valencia & Catalana & Mapfre & MSCI \\
\hline Mean & 0.000 & 0.000 & 0.000 & -0.001 & 0.000 & 0.000 & -0.001 & 0.001 & 0.000 & 0.000 \\
Std. Dev. & 0.022 & 0.025 & 0.029 & 0.025 & 0.020 & 0.033 & 0.037 & 0.022 & 0.022 & 0.021 \\
Maximum & 0.183 & 0.151 & 0.365 & 0.203 & 0.175 & 0.608 & 0.668 & 0.132 & 0.134 & 0.154 \\
Minimum & -0.235 & -0.092 & -0.163 & -0.122 & -0.118 & -0.293 & -0.334 & -0.120 & -0.157 & -0.116 \\
Skewness & -0.018 & 0.477 & 1.026 & 0.751 & 0.796 & 2.720 & 2.348 & 0.269 & 0.118 & 0.421 \\
Kurtosis & 14.602 & 5.776 & 19.666 & 10.360 & 12.184 & 60.472 & 65.466 & 6.393 & 7.822 & 9.423 \\
J-B & $13449^{*}$ & $860.89^{*}$ & $28174^{*}$ & $5638.4 *$ & $8681.9^{*}$ & $332978^{*}$ & $392072^{*}$ & $1179.3^{*}$ & $2328.7^{*}$ & $4192.8^{*}$ \\
Q(20) & 39.184 & 44.197 & 74.862 & 55.902 & 77.119 & 100.520 & 128.850 & 42.193 & 52.843 & 55.391 \\
& {$[0.006]$} & {$[0.001]$} & {$[0.000]$} & {$[0.000]$} & {$[0.000]$} & {$[0.000]$} & {$[0.000]$} & {$[0.003]$} & {$[0.000]$} & {$[0.000]$} \\
ARCH & 3.448 & 12.133 & 28.833 & 11.042 & 7.861 & 30.954 & 16.363 & 12.475 & 13.934 & 16.218 \\
& {$[0.000]$} & {$[0.000]$} & {$[0.000]$} & {$[0.000]$} & {$[0.000]$} & {$[0.000]$} & {$[0.000]$} & {$[0.000]$} & {$[0.000]$} & {$[0.000]$} \\
Pearson Corr. & 0.693 & 0.700 & 0.934 & 0.806 & 0.708 & 0.891 & 0.318 & 0.469 & 0.629 & \\
\hline
\end{tabular}

Note. Daily data for the period 16 January 2003 to 28 February 2013. J-B denote the Jarque-Bera statistic for normality. $\mathrm{Q}(k)$ is the Ljung-Box statistics for serial correlation in squared returns computed with $k$ lags. ARCH denotes Engle's LM test for heteroskedasticity computed using 20 lags. Pearson Corr. is the Pearson correlation between the financial system (MSCI) and each financial institution. An asterisk $(*)$ indicates rejection of the null hypothesis at $5 \%$. 
Table 2.2 shows that weekly returns for the financial institutions had similar characteristics. Specifically, returns on average were approximately zero and their standard deviations were larger in an order of several magnitudes, hence, no significant trend was observed in the returns. The Banco de Valencia showed greater volatility than the other institutions, in terms of the standard deviation and the distance between maximum and minimum values. For all the series the Jarque-Bera test clearly rejected the null hypothesis of normality. Asymmetry and fat tails in the returns were evident in the fact that the kurtosis coefficient was above 3 . The autoregressive conditional heteroskedasticity-Lagrange multiplier (ARCH-LM) statistic indicated that all the return series displayed ARCH effects. Finally, the Pearson's correlation coefficient for each financial institution with the system was positive, with larger institutions taking greater values.

\subsection{Results}

Below we present the marginal model and VaR results for each financial institution and then report the CoVaR results obtained using the different approaches.

\subsubsection{Marginal model and $\mathrm{VaR}$ results}

The estimation results for the marginal models reflected in Eqs. (2.4)-(2.6) are reported in Table 2.4 for all the institutions and overall financial system returns. We considered different combinations of the parameters $\mathrm{p}, \mathrm{r}$ and $\mathrm{m}$ for values ranging from zero to a maximum lag of two, selecting the most suitable value according to the BIC and AIC. We can summarize the evidence on the marginal models as follows. Average returns exhibited, in general, no temporal dependence and returns volatility was persistent across different financial institutions, with some volatility dynamics (for Banesto, Banco Popular, Banco de Valencia and Catalana Occidente) described by several lags in the TGARCH model specification. Leverage effects were also found for all series with the exception of Banco Popular and Banco de Valencia. The estimated degrees of freedom and symmetry parameter values for the skewed Student-t distribution confirm that the error terms were not normal and were even asymmetric in two cases. 
Table 2.4: Maximum likelihood estimates.

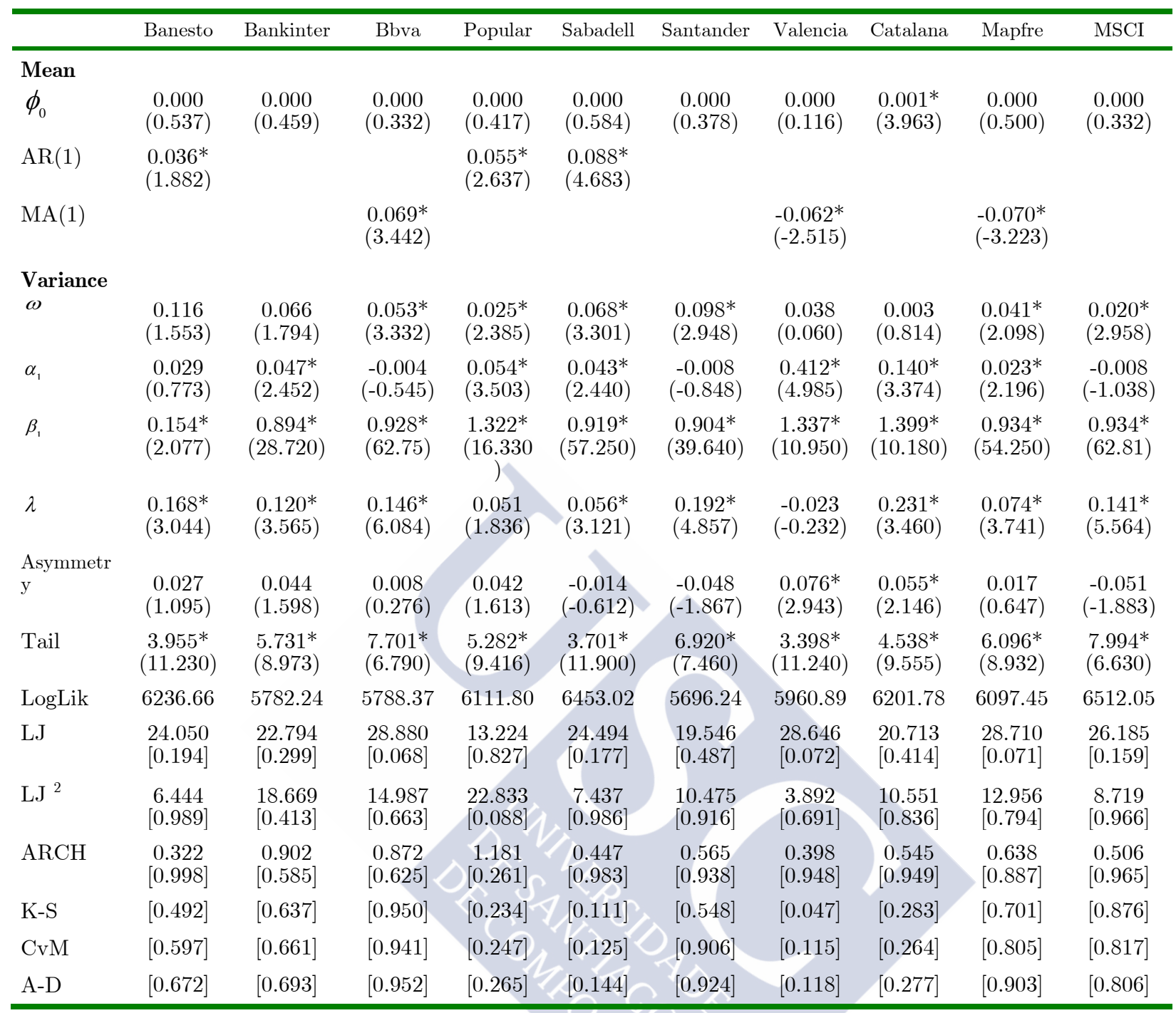

Note. The table presents the coefficients of the maximum likelihood (ML) estimates and the $\mathrm{z}$ statistics (in parentheses) for the parameters of the marginal distribution used to calculate the volatility of the series and to calculate the dynamic conditional correlation (DCC) model. LogLik is the log-likelihood value. LJ represents the Ljung-Box statistic for serial correlation in the residual model calculated with 20 lags. LJ ${ }^{2}$ represents the Ljung-Box statistic for serial correlation in the squared residual model calculated with 20 lags. ARCH is Engle's LM test for the ARCH effect in the residuals up to 20th order. K-S, CvM and A-D denote the Kolmogorov-Smirnov, Cramér-von Mises and Anderson-Darling test for adequacy of the skewed-t distribution model. $\mathrm{P}$ values (in square brackets) below 0.05 indicate rejection of the null hypothesis. An asterisk $\left(^{*}\right)$ indicates significance at $5 \%$. For specific institutions the best models are as follows: Banesto, TGARCH(1,2); Banco Popular, $\operatorname{TGARCH}(2,3)$; and Banco de Valencia and Catalana Occidente, TGARCH(2,1). 
The last rows of Table 2.4 report information on the goodness-of-fit of our marginal models. The Ljung-Box and $\mathrm{ARCH}$ statistics indicate that neither autocorrelation nor ARCH effects remained in the residuals of the marginal models. We also tested the null hypothesis that the standardized model residuals were uniform $(0,1)$ by comparing the empirical distribution with the theoretical distribution function using the well-known Kolmogorov-Smirnov (K-S), Cramér-von Mises (CvM) and Anderson-Darling (AD) tests. The p-values for these tests, reported in last three rows of Table 2.4, indicate that, for either of the marginal models, the null of the correct specification of the distribution function could not be rejected at the $5 \%$ significance level. Overall, our goodness-of-fit tests indicate that the marginal distribution models were not mis-specified.

We computed the $\mathrm{VaR}$ for the $95 \%$ confidence level ${ }^{4}$ for each financial institution using information from its marginal model and Eq. (2.7). Figure 2.3 depicts the temporal dynamics of the VaR for all the institutions, which showed similar trends throughout the sample period, with the VaR decreasing considerably during the recent global financial crisis and less intensely during the European sovereign debt crisis. Also, due to financial difficulties experienced at the end of the sampling period, the VaR value for the Banco de Valencia turned down since 2011. Table 2.5 reports descriptive statistic for the VaR. Larger banks like Santander and BBVA had larger mean VaR values (in absolute terms), although not Banco de Valencia, given that it was experiencing financial distress. Likewise, VaR volatility increased with institution size.

Table 2.5: Descriptive statistics for the value-at-risk at the $95 \%$ confidence level.

\begin{tabular}{lccccccccc}
\hline & Banesto & Bankinter & Bbva & Popular & Sabadell & Santander & Valencia & Catalana & Mapfre \\
\hline Mean & -0.031 & -0.037 & -0.039 & -0.034 & -0.028 & -0.042 & -0.041 & -0.032 & -0.033 \\
Std. Dev. & 0.013 & 0.015 & 0.023 & 0.018 & 0.010 & 0.027 & 0.041 & 0.016 & 0.013 \\
Maximum & -0.016 & -0.016 & -0.015 & -0.011 & -0.015 & -0.017 & -0.013 & -0.007 & -0.016 \\
Minimum & -0.169 & -0.087 & -0.188 & -0.102 & -0.077 & -0.275 & -0.647 & -0.123 & -0.105 \\
\hline
\end{tabular}

\footnotetext{
${ }^{4}$ Results for the $97 \%$ and $99 \%$ confidence levels are available on request.
} 
Figure 2.3: Time series plot for value-at-risk at the $95 \%$ confidence level.

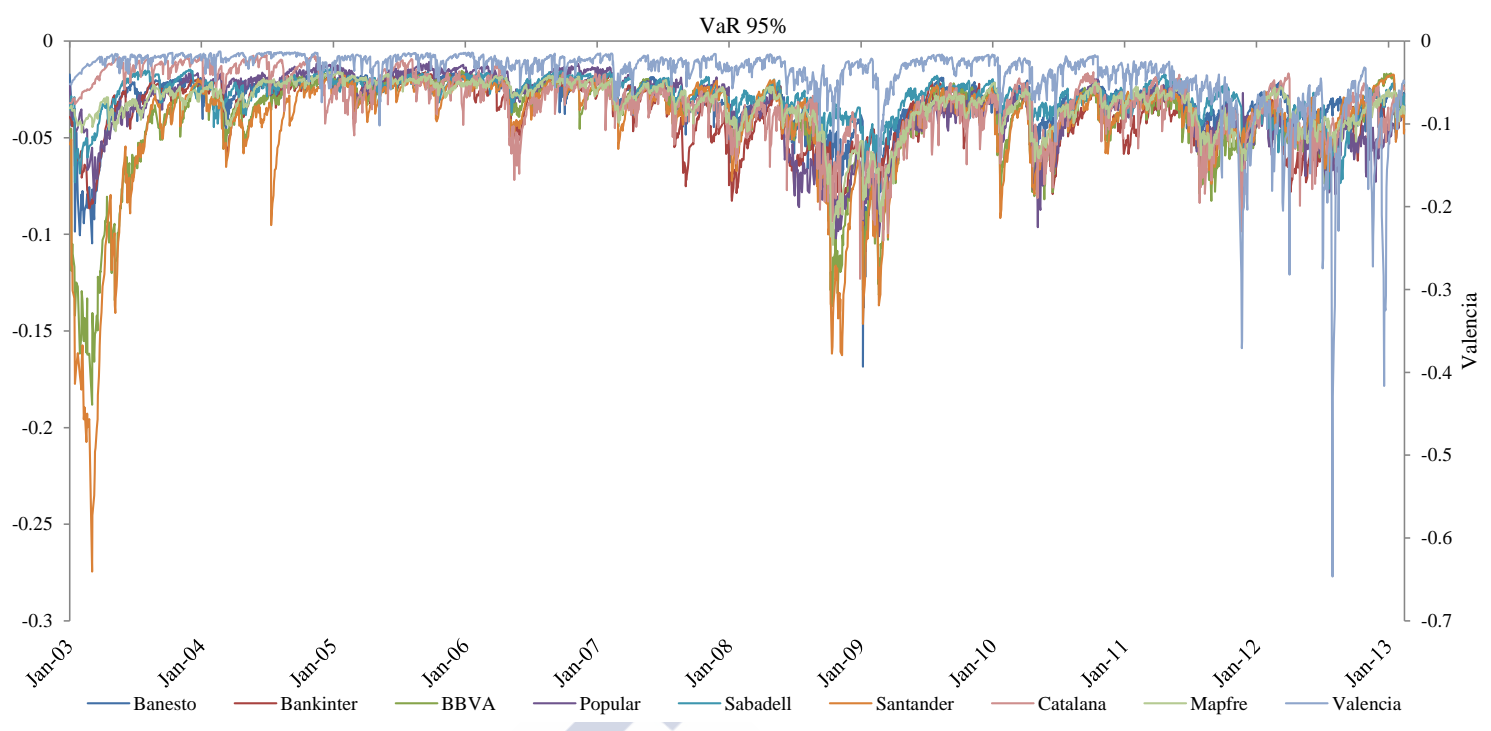

\subsubsection{Quantile regression results}

With the value of the VaR for each financial institution and the set of explanatory variables described in the data section, we estimated the CoVaR for the financial system using the quantile regression reflected in Eq. (2.3). Table 2.6 reports estimates for a $95 \%$ confidence level $(1-\beta=95 \%$ and $1-\alpha=95 \%)$. Given that the quantil regression parameter $\beta$ was significant and had the expected sign for all the financial institutions, the VaR for each institution had a significant impact on the VaR of the financial system. Regarding the explanatory variables, we found that market volatility and yield curve slope were significant and had the expected sign in all cases; other explanatory variables were only significant in some cases; and the IBEX-35 returns were non-significant in all the cases.

For each financial institution the CoVaR value at each time $t$ was computed as the estimated value of the corresponding quantile regression. Table 2.9 reports descriptive statistics for the CoVaR, showing values that were greater for the banks than for the insurance companies and, in general, with values reflecting the size of the bank. Likewise, larger banks generated more instability in CoVaR values than small banks or insurance companies. 
Table 2.6: Quantile regression estimates at the $95 \%$ confidence level.

\begin{tabular}{ccccccc}
\hline & $\beta$ & $\Psi$ & $\Phi$ & $\rho$ & $\zeta$ & $\lambda$ \\
\hline \multirow{2}{*}{ Banesto } & $0.834^{* * *}$ & $-0.091^{* * *}$ & -0.114 & $-0.156^{* * *}$ & -0.072 & -0.008 \\
& $(0.058)$ & $(0.006)$ & $(0.219)$ & $(0.035)$ & $(0.110)$ & $(0.023)$ \\
\hline \multirow{2}{*}{ Bankinter } & $0.637^{* * *}$ & $-0.111^{* * *}$ & 0.179 & $-0.188^{* * *}$ & $0.591^{* * *}$ & -0.013 \\
& $(0.049)$ & $(0.008)$ & $(0.316)$ & $(0.034)$ & $(0.101)$ & $(0.022)$ \\
\hline \multirow{2}{*}{ BBVA } & $0.790^{* * *}$ & $-0.026^{* * *}$ & 0.051 & $-0.100^{* * *}$ & $-0.202^{* * *}$ & -0.014 \\
& $(0.021)$ & $(0.003)$ & $(0.096)$ & $(0.014)$ & $(0.058)$ & $(0.009)$ \\
\hline \multirow{2}{*}{ Popular } & $0.766^{* * *}$ & $-0.061^{* * *}$ & 0.059 & 0.004 & -0.013 & -0.038 \\
& $(0.085)$ & $(0.012)$ & $(0.375)$ & $(0.050)$ & $(0.213)$ & $(0.027)$ \\
\hline \multirow{2}{*}{ Sabadell } & $0.719^{* * *}$ & $-0.134^{* * *}$ & 0.049 & $-0.147^{* * *}$ & $0.379^{* *}$ & -0.022 \\
& $(0.048)$ & $(0.006)$ & $(0.299)$ & $(0.034)$ & $(0.175)$ & $(0.023)$ \\
\hline \multirow{2}{*}{ Santander } & $0.634^{* * *}$ & $-0.054^{* * *}$ & $-0.288^{* * *}$ & $-0.131^{* * *}$ & $-0.447^{* * *}$ & 0.001 \\
& $(0.026)$ & $(0.004)$ & $(0.109)$ & $(0.020)$ & $(0.044)$ & $(0.011)$ \\
\hline \multirow{2}{*}{ Valencia } & $0.145^{* * *}$ & $-0.183^{* * *}$ & -0.011 & $-0.177^{* * *}$ & $0.669^{* * *}$ & -0.005 \\
& $(0.025)$ & $(0.007)$ & $(0.413)$ & $(0.033)$ & $(0.143)$ & $(0.025)$ \\
\hline \multirow{2}{*}{ Catalana } & $0.272^{* * *}$ & $-0.161^{* * *}$ & 0.084 & $-0.306^{* * *}$ & $0.485^{* * *}$ & -0.007 \\
& $(0.042)$ & $(0.012)$ & $(0.615)$ & $(0.038)$ & $(0.178)$ & $(0.028)$ \\
\hline \multirow{2}{*}{ Mapfre } & $1.017^{* * *}$ & $-0.043^{* * *}$ & -0.219 & $-0.291^{* * *}$ & 0.017 & -0.008 \\
& $(0.087)$ & $(0.010)$ & $(0.368)$ & $(0.034)$ & $(0.110)$ & $(0.021)$ \\
\hline
\end{tabular}

Note.

The quantile regression coefficients,

$Q(\tau / x)=\alpha+\beta^{s i} \operatorname{VaR}_{t, p}^{i}+\psi^{s i} M_{k V o l}^{i} t_{t-1, p}+\phi^{s i} \Delta \operatorname{TrBill12} 2_{t-1, p}^{i}+\rho^{s i}$ Slope $_{t-1, p}^{i}+\varsigma^{s i}$ cr.spread $_{t-1, p}^{i}+\lambda^{s i}$ Mkreturns $_{t-1, p}^{i}$,

$\tau=0.01$, are as follows:

$\beta=$ coefficient of the quantile regression for the returns of each institution $\left(X_{t}^{i}\right)$,

$\psi=$ coefficient of the quantile regression for market volatility (IBEX-35),

$\phi=$ coefficient of the quantile regression for the 12-month Treasury bill variation rate,

$\rho=$ coefficient of the quantile regression for the yield curve slope,

$\zeta=$ coefficient of the quantile regression for the credit spread,

$\lambda=$ coefficient of the quantile regression for the market returns (IBEX-35)

Numbers in brackets indicate standard error:

* Significance at $10 \%$.

** Significance at $5 \%$.

*** Significance at $1 \%$.

\subsubsection{MGARCH results}

We estimated the DCC model for returns composed of the financial system paired with each financial institution. Table 2.7 reports the results for the parameters and degrees of freedom, as reflected in Eq. (2.11), for the bivarate Student-t distribution. All the parameters were significant, providing consistent evidence in favour of timevarying dependence and fat tails according to the estimated degrees of freedom. Figure 2.4 depicts correlation dynamics for each financial institution, showing that institutional interdependence with the general financial system varies according to the institution's size, with no significant change with the onset of the global 
financial crisis or of the European sovereign debt crisis. With this correlation coefficient and marginal model information we have all the information on the joint distribution function of the financial system and the financial institution at each time t. Hence, taking the VaR of a given financial institution computed through its marginal return model, we can compute the CoVaR value by numerically solving Eq. (2.10) for the CoVaR and $1-\beta=95 \%$ and $1-\alpha=95 \%$.

Table 2.7: Dynamic conditional correlation (DCC) model estimates.

\begin{tabular}{cccccccccc}
\hline & Banesto & Bankinter & BBVA & Popular & Sabadell & Santander & Valencia & Catalana & Mapfre \\
\hline \multirow{2}{*}{ DoF } & $5.220^{*}$ & $6.110^{*}$ & $6.270^{*}$ & $5.682^{*}$ & $4.850^{*}$ & $5.139^{*}$ & $4.900^{*}$ & $6.294^{*}$ & 7.185 \\
& $(15.65)$ & $(14.61)$ & $(13.91)$ & $(15.21)$ & $(18.27)$ & $(17.21)$ & $(19.49)$ & $(13.59)$ & $(11.42)$ \\
$\alpha$ & $0.056^{*}$ & $0.056^{*}$ & $0.044^{*}$ & $0.038^{*}$ & $0.030^{*}$ & $0.035^{*}$ & $0.017^{*}$ & $0.010^{*}$ & $0.025^{*}$ \\
& $(3.71)$ & $(5.50)$ & $(3.73)$ & $(5.23)$ & $(3.11)$ & $(3.74)$ & $(3.20)$ & $(3.75)$ & $(3.11)$ \\
\multirow{2}{*}{$\beta$} & $0.917^{*}$ & $0.696^{*}$ & $0.659^{*}$ & $0.696^{*}$ & $0.638^{*}$ & $0.692^{*}$ & $0.686^{*}$ & $0.689^{*}$ & $0.673^{*}$ \\
& $(33.10)$ & $(51.49)$ & $(42.56)$ & $(90.82)$ & $(71.76)$ & $(77.19)$ & $(175.60)$ & $(314.30)$ & $(59.91)$ \\
\hline
\end{tabular}

Note. In accordance with the DCC model, $\alpha$ and $\beta$ are the parameters estimated with the Student-t distributed errors. An asterisk $(*)$ indicates rejection of the null hypothesis at $5 \%$.

Figure 2.4: Time series plot for dynamic conditional correlation (DCC).

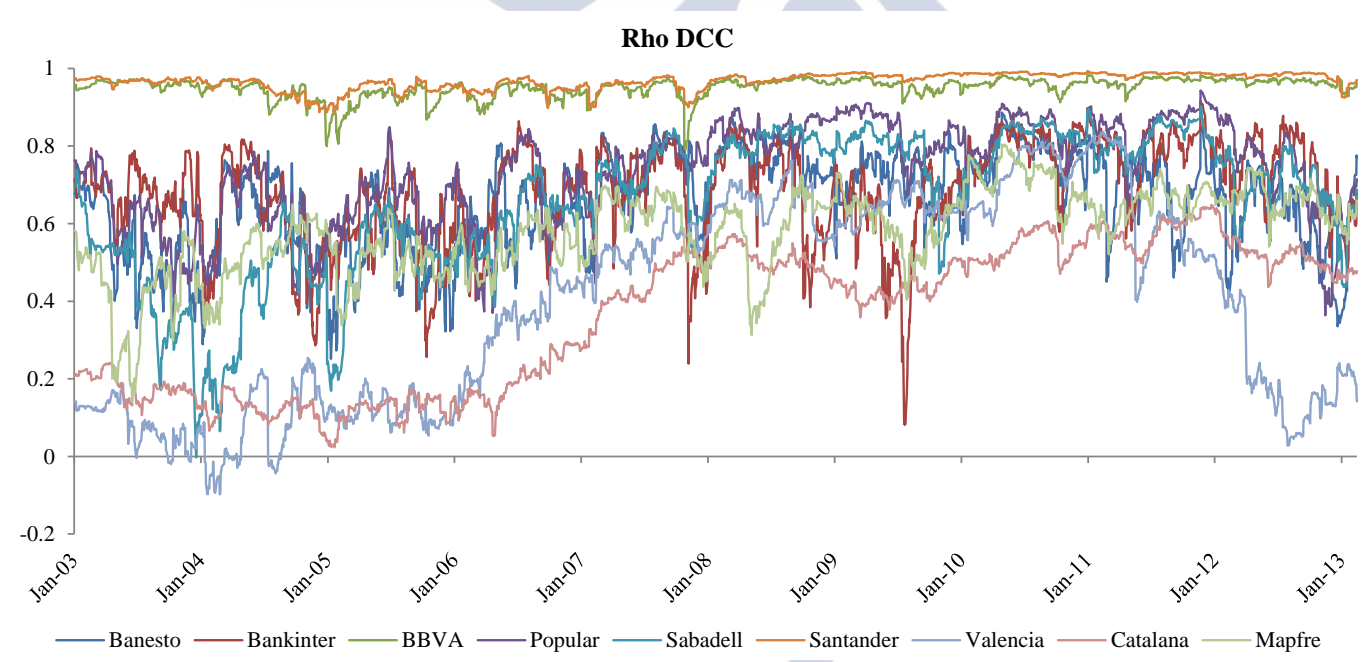

Descriptive statistics for the CoVaR values are reported in Table 2.9. The evidence on systemic risk is qualitatively similar to that reported for the quantile regression approach, even though the size of the CoVaR was quite different. The CoVaR values obtained using the MGARCH approach were considerably reduced with respect to those obtained using the quantile regression approach. This is consistent with the idea that conditioning on the fact that $\mathrm{X}_{\mathrm{t}}^{\mathrm{i}} \leq \operatorname{VaR}_{\mathrm{t}, \alpha}^{\mathrm{i}}$ and not on the fact that $\mathrm{X}_{\mathrm{t}}^{\mathrm{i}}=\mathrm{VaR}_{\mathrm{t}, \alpha}^{\mathrm{i}}$ has a quantitative impact on the value of the CoVaR (an effect that was also discussed by Girardi and Ergün, 2013). 


\subsubsection{Copula results}

Table 2.8 reports parameter estimates for the nine pairs given by the MSCI Spain Financials Index returns matched with each institution's returns. Both static and dynamic copula specifications indicate that all the financial institutions co-moved with the general financial index. Parameter estimates for all the static copulas were significant, as also was the case for most of the time-varying copula specifications. Considering the AIC values corrected for small-sample bias, we found evidence of time-varying dependence and lower tail dependence, with the time-varying rotated Gumbel copula offering the best fit for all institutions, except for BBVA and Catalana Occidente, for which the time-varying Student-t performed better, thereby providing evidence of symmetric tail dependence.

From the best copula specification and following the same two-step procedure as described above, we obtained the CoVaR value at the $95 \%$ confidence level for the financial system conditional on the $\mathrm{VaR}$ of each institution at the $95 \%$ confidence level. We also obtained the $\Delta \mathrm{CoVaR}$ value using Eq. (2.2). Figure 2.5 depicts the dynamic behaviour of the estimated $\mathrm{CoVaR}$ and $\Delta \mathrm{CoVaR}$ (in percentage) and reports - to enable assessment and comparison of the impact of different methodological approaches - the CoVaR values estimated using the quantile regression and MGARCH approaches. The graphical evidence for the CoVar points to two conclusions. First, trends in the CoVaR values were consistent across different financial institutions, with systemic risk increasing around the onset of the global financial and the European sovereign debt crises. Significant changes also occurred in the CoVaR values in April 2011, when the systemic risk of Spanish financial institutions increased due to Portugal requesting a financial bailout. Second, there were significant differences in $\mathrm{CoVaR}$ values obtained using quantile regression as compared to using the MGARCH and copula approaches. Specifically, quantile regression $\mathrm{CoVaR}$ values were higher and also relatively stable through the sampling period, with little variation once the crises broke, indicating that the real systemic risk was underestimated. In contrast, MGARCH and copula CoVaR values were lower and reflected dynamics that adjusted much better to crisis events; thus, values were reduced when the crises broke and increased in more stable periods, thereby indicating a better adjustment to the changing economic environment in the latter years of the sampling period. 
Table 2.8: Copula model estimates for institutions vs the MSCI Index.

Panel A: Parameter estimates for time-invariant copulas

\begin{tabular}{|c|c|c|c|c|c|c|c|c|c|}
\hline Copula & Banesto & Bankinter & Bbva & Popular & Sabadell & Santander & Valencia & Catalana & Mapfre \\
\hline \multicolumn{10}{|c|}{ Gaussian } \\
\hline$\rho$ & 0.661 & 0.680 & 0.947 & 0.742 & 0.666 & 0.967 & 0.438 & 0.404 & 0.586 \\
\hline AIC & -1375.837 & -1485.457 & -5458.490 & -1918.773 & -1404.743 & -6575.424 & -508.015 & -424.749 & -1005.770 \\
\hline \multicolumn{10}{|c|}{ Student-t } \\
\hline$\rho$ & $\begin{array}{r}0.667^{*} \\
(0.01)\end{array}$ & $\begin{array}{r}0.692^{*} \\
(0.01)\end{array}$ & $\begin{array}{r}0.951^{*} \\
(0.00)\end{array}$ & $\begin{array}{r}0.755^{*} \\
(0.01)\end{array}$ & $\begin{array}{r}0.684^{*} \\
(0.01)\end{array}$ & $\begin{array}{c}0.970^{*} \\
(0.00)\end{array}$ & $\begin{array}{r}0.459^{*} \\
(0.02)\end{array}$ & $\begin{array}{r}0.411^{*} \\
(0.01)\end{array}$ & $\begin{array}{r}0.589^{*} \\
(0.01)\end{array}$ \\
\hline$v$ & $\begin{array}{r}6.175^{*} \\
(1.95)\end{array}$ & $\begin{array}{c}5.300^{*} \\
(1.47)\end{array}$ & $\begin{array}{r}4.055^{*} \\
(0.48)\end{array}$ & $\begin{array}{r}4.907^{*} \\
(0.46)\end{array}$ & $\begin{array}{r}4.065^{*} \\
(0.41)\end{array}$ & $\begin{array}{r}3.389^{*} \\
(0.36)\end{array}$ & $\begin{array}{r}5.426^{*} \\
(0.74)\end{array}$ & $\begin{array}{r}12.943^{*} \\
(1.81)\end{array}$ & $\begin{array}{r}8.622^{*} \\
(0.87)\end{array}$ \\
\hline $\mathrm{AIC}$ & -1434.531 & -1581.141 & -5714.405 & -2037.900 & -1568.800 & -6861.588 & -588.128 & -437.843 & -1043.008 \\
\hline \multicolumn{10}{|l|}{ Gumbel } \\
\hline$\delta$ & $\begin{array}{r}1.749^{*} \\
(0.03)\end{array}$ & $\begin{array}{r}1.813^{*} \\
(0.03)\end{array}$ & $\begin{array}{c}4.629^{*} \\
(0.08)\end{array}$ & $\begin{array}{r}2.057^{*} \\
(0.03)\end{array}$ & $\begin{array}{r}1.802^{*} \\
(0.03)\end{array}$ & $\begin{array}{c}5.860^{*} \\
(0.10)\end{array}$ & $\begin{array}{r}1.385^{*} \\
(0.02)\end{array}$ & $\begin{array}{r}1.314^{*} \\
(0.02)\end{array}$ & $\begin{array}{r}1.578^{*} \\
(0.03)\end{array}$ \\
\hline AIC & -1205.439 & -1324.791 & -5320.482 & -1797.433 & -1298.704 & -6361.999 & -469.609 & -356.186 & -883.238 \\
\hline \multicolumn{10}{|c|}{ Rotated Gumbel } \\
\hline$\delta$ & $\begin{array}{r}1.838^{*} \\
(0.03)\end{array}$ & $\begin{array}{r}1.915^{*} \\
(0.03)\end{array}$ & $\begin{array}{r}4.794^{*} \\
(0.08)\end{array}$ & $\begin{array}{r}2.125^{*} \\
(0.04)\end{array}$ & $\begin{array}{r}1.903^{*} \\
(0.03)\end{array}$ & $\begin{array}{r}6.197^{*} \\
(0.11)\end{array}$ & $\begin{array}{r}1.411^{*} \\
(0.02)\end{array}$ & $\begin{array}{r}1.336^{*} \\
(0.02)\end{array}$ & $\begin{array}{r}1.623^{*} \\
(0.03)\end{array}$ \\
\hline $\mathrm{AIC}$ & -1460.203 & -1594.086 & -5528.936 & -1983.757 & -1565.309 & -6685.885 & -568.721 & -422.484 & -1019.961 \\
\hline \multicolumn{10}{|l|}{ BB7 } \\
\hline$\theta$ & $\begin{array}{r}1.428^{*} \\
(0.04)\end{array}$ & $\begin{array}{c}1.454^{*} \\
(0.05)\end{array}$ & $\begin{array}{r}4.034^{*} \\
(0.00)\end{array}$ & $\begin{array}{l}1.738^{*} \\
(0.08)\end{array}$ & $\begin{array}{r}1.448^{*} \\
(0.09)\end{array}$ & $\begin{array}{l}4.103^{*} \\
(0.04)\end{array}$ & $\begin{array}{r}1.233^{*} \\
(0.03)\end{array}$ & $\begin{array}{r}1.185^{*} \\
(0.03)\end{array}$ & $\begin{array}{r}1.369^{*} \\
(0.04)\end{array}$ \\
\hline$\delta$ & $\begin{array}{r}1.126^{*} \\
(0.05)\end{array}$ & $\begin{array}{c}1.217^{*} \\
(0.05)\end{array}$ & $\begin{array}{r}4.263^{*} \\
(0.00)\end{array}$ & $\begin{array}{l}1.327^{*} \\
(0.06)\end{array}$ & $\begin{array}{r}1.218^{*} \\
(0.04)\end{array}$ & $\begin{array}{r}6.311^{*} \\
(0.21)\end{array}$ & $\begin{array}{r}0.547^{*} \\
(0.04)\end{array}$ & $\begin{array}{r}0.453^{*} \\
(0.04)\end{array}$ & $\begin{array}{r}0.798^{*} \\
(0.04)\end{array}$ \\
\hline $\mathrm{AIC}$ & -1431.329 & -1552.662 & -5355.069 & -1931.303 & -1530.783 & -6214.699 & -551.679 & -415.580 & -1018.554 \\
\hline \multicolumn{10}{|c|}{ Plackett } \\
\hline$\theta$ & $\begin{array}{r}9.732^{*} \\
(0.52)\end{array}$ & $\begin{array}{r}11.279^{*} \\
(0.57)\end{array}$ & $\begin{array}{r}99.679^{*} \\
(4.53)\end{array}$ & $\begin{array}{r}15.385 * \\
(0.75)\end{array}$ & $\begin{array}{r}11.115^{*} \\
(0.57)\end{array}$ & $\begin{array}{r}186.395^{*} \\
(29.81)\end{array}$ & $\begin{array}{c}4.516^{*} \\
(0.26)\end{array}$ & $\begin{array}{c}3.581^{*} \\
(0.20)\end{array}$ & $\begin{array}{r}6.682^{*} \\
(0.35)\end{array}$ \\
\hline $\mathrm{AIC}$ & -1387.328 & -1567.059 & -5534.123 & -2016.034 & -1525.218 & -6787.020 & -568.881 & -428.177 & -981.821 \\
\hline \multicolumn{10}{|l|}{ BB1 } \\
\hline$\theta$ & $\begin{array}{c}0.721^{*} \\
(0.05)\end{array}$ & $\begin{array}{r}0.753^{*} \\
(0.06)\end{array}$ & $\begin{array}{r}0.906^{*} \\
(0.04)\end{array}$ & $\begin{array}{r}0.659^{*} \\
(0.05)\end{array}$ & $\begin{array}{r}0.763^{*} \\
(0.06)\end{array}$ & $\begin{array}{r}1.036^{*} \\
(0.07)\end{array}$ & $\begin{array}{r}0.383^{*} \\
(0.04)\end{array}$ & $\begin{array}{r}0.322^{*} \\
(0.04)\end{array}$ & $\begin{array}{r}0.515^{*} \\
(0.05)\end{array}$ \\
\hline$\delta$ & $\begin{array}{r}1.348^{*} \\
(0.03)\end{array}$ & $\begin{array}{r}1.384^{*} \\
(0.03)\end{array}$ & $\begin{array}{r}3.352^{*} \\
(0.05)\end{array}$ & $\begin{array}{r}1.612^{*} \\
(0.04)\end{array}$ & $\begin{array}{r}1.372^{*} \\
(0.03)\end{array}$ & $\begin{array}{r}4.090^{*} \\
(0.12)\end{array}$ & $\begin{array}{r}1.195^{*} \\
(0.03)\end{array}$ & $\begin{array}{c}1.161^{*} \\
(0.02)\end{array}$ & $\begin{array}{r}1.300^{*} \\
(0.03)\end{array}$ \\
\hline $\mathrm{AIC}$ & -1459.027 & -1590.462 & -5646.838 & -2004.665 & -1562.220 & -6754.743 & -563.207 & -426.512 & -1039.673 \\
\hline
\end{tabular}


Panel B: Parameter estimates for time-varying copulas.

\begin{tabular}{|c|c|c|c|c|c|c|c|c|c|}
\hline & Banesto & Bankinter & Bbva & Popular & Sabadell & Santander & Valencia & Catalana & Mapfre \\
\hline \multicolumn{10}{|c|}{ TVP-Gaussian } \\
\hline \multirow[t]{2}{*}{$\psi_{0}$} & -0.269 & 0.076 & -1.294 & $-0.517^{*}$ & $-0.039^{*}$ & 5.000 & 0.030 & -0.041 & $-0.144^{*}$ \\
\hline & $(1.67)$ & $(0.13)$ & $(9.37)$ & $(0.10)$ & $(0.00)$ & $(9.58)$ & $(0.02)$ & $(0.92)$ & $(0.07)$ \\
\hline \multirow[t]{2}{*}{$\psi_{1}$} & 0.176 & $0.300^{*}$ & 0.201 & $0.184^{*}$ & $0.262^{*}$ & 0.116 & $0.241^{*}$ & 0.045 & $0.083^{*}$ \\
\hline & $(5.81)$ & $(0.06)$ & $(0.18)$ & $(0.04)$ & $(0.01)$ & $(2.35)$ & $(0.05)$ & $(2.46)$ & $(0.03)$ \\
\hline \multirow{2}{*}{$\psi_{2}$} & 2.695 & $2.070^{*}$ & 5.000 & $3.121^{*}$ & $2.286^{*}$ & -1.022 & $1.909^{*}$ & 2.200 & $2.469^{*}$ \\
\hline & $(3.73)$ & $(0.24)$ & $(10.05)$ & $(0.17)$ & $(0.02)$ & $(6.16)$ & $(0.08)$ & $(1.49)$ & $(0.15)$ \\
\hline $\mathrm{AIC}$ & -1433.80 & -1552.50 & -5471.60 & -1983.83 & -1554.62 & -6575.19 & -636.04 & -473.50 & -1028.27 \\
\hline \multicolumn{10}{|c|}{ TVP-Student-t } \\
\hline$\psi_{0}$ & $\begin{array}{r}-0.198^{*} \\
(0.02)\end{array}$ & $\begin{array}{c}-0.072 \\
(0.11)\end{array}$ & $\begin{array}{r}-14.21^{*} \\
(0.00)\end{array}$ & $\begin{array}{r}-0.657^{*} \\
(0.08)\end{array}$ & $\begin{array}{r}-0.037^{*} \\
(0.00)\end{array}$ & $\begin{array}{r}-12.743^{*} \\
(5.05)\end{array}$ & $\begin{array}{c}0.059 \\
(0.05)\end{array}$ & $\begin{array}{c}0.003 \\
(0.02)\end{array}$ & $\begin{array}{l}2.183 \\
(2.03)\end{array}$ \\
\hline$\psi_{1}$ & $\begin{array}{r}0.131^{*} \\
(0.02)\end{array}$ & $\begin{array}{r}0.156^{*} \\
(0.03)\end{array}$ & $\begin{array}{l}0.03^{*} \\
(0.00)\end{array}$ & $\begin{array}{r}0.103^{*} \\
(0.03)\end{array}$ & $\begin{array}{c}0.189^{*} \\
(0.02)\end{array}$ & $\begin{array}{c}-0.014 \\
(0.01)\end{array}$ & $\begin{array}{r}0.205^{*} \\
(0.04)\end{array}$ & $\begin{array}{c}0.097^{*} \\
(0.03)\end{array}$ & $\begin{array}{c}0.249 \\
(0.14)\end{array}$ \\
\hline$\psi_{2}$ & $\begin{array}{r}2.543^{*} \\
(0.04)\end{array}$ & $\begin{array}{r}2.388^{*} \\
(0.19)\end{array}$ & $\begin{array}{r}18.80^{*} \\
(0.00)\end{array}$ & $\begin{array}{c}3.354^{*} \\
(0.14)\end{array}$ & $\begin{array}{r}2.297^{*} \\
(0.01)\end{array}$ & $\begin{array}{r}17.521^{*} \\
(5.20)\end{array}$ & $\begin{array}{r}1.843^{*} \\
(0.15)\end{array}$ & $\begin{array}{r}2.034^{*} \\
(0.08)\end{array}$ & $\begin{array}{r}-1.707 \\
(3.55)\end{array}$ \\
\hline$v$ & $\begin{array}{r}6.221^{*} \\
(0.46)\end{array}$ & $\begin{array}{r}6.677^{*} \\
(1.14)\end{array}$ & $\begin{array}{c}5.00^{*} \\
(0.00)\end{array}$ & $\begin{array}{c}5.770^{*} \\
(0.35)\end{array}$ & $\begin{array}{c}6.666^{*} \\
(0.68)\end{array}$ & $\begin{array}{r}3.171^{*} \\
(0.33)\end{array}$ & $\begin{array}{l}7.646^{*} \\
(2.84)\end{array}$ & $\begin{array}{r}17.118^{*} \\
(2.18)\end{array}$ & $\begin{array}{r}9.488^{*} \\
(1.93)\end{array}$ \\
\hline $\mathrm{AIC}$ & -1480.20 & -1618.48 & -5736.39 & -2085.60 & -1642.09 & -6861.18 & -684.12 & -481.04 & -1046.55 \\
\hline \multicolumn{10}{|c|}{ TVP-Gumbel } \\
\hline$\omega$ & $\begin{array}{r}0.772^{*} \\
(0.32)\end{array}$ & $\begin{array}{l}0.595 \\
(0.38)\end{array}$ & $\begin{array}{r}1.768^{*} \\
(0.17)\end{array}$ & $\begin{array}{r}0.946^{*} \\
(0.43)\end{array}$ & $\begin{array}{c}1.121^{*} \\
(0.24)\end{array}$ & $\begin{array}{l}1.417^{*} \\
(0.04)\end{array}$ & $\begin{array}{r}0.675^{*} \\
(0.12)\end{array}$ & $\begin{array}{r}0.389 \\
(0.36)\end{array}$ & $\begin{array}{r}1.505^{*} \\
(0.20)\end{array}$ \\
\hline $\bar{\beta}$ & $\begin{array}{l}0.222 \\
(0.12)\end{array}$ & $\begin{array}{c}0.300^{*} \\
(0.13)\end{array}$ & $\begin{array}{r}0.106^{*} \\
(0.03)\end{array}$ & $\begin{array}{r}0.198 \\
(0.14)\end{array}$ & $\begin{array}{l}0.147 \\
(0.08)\end{array}$ & $\begin{array}{c}0.167^{*} \\
(0.00)\end{array}$ & $\begin{array}{r}0.279^{*} \\
(0.05)\end{array}$ & $\begin{array}{r}0.329 \\
(0.19)\end{array}$ & $\begin{array}{r}-0.194 \\
(0.11)\end{array}$ \\
\hline $\bar{\alpha}$ & $\begin{array}{r}-1.626^{*} \\
(0.58)\end{array}$ & $\begin{array}{r}-1.390 \\
(0.77)\end{array}$ & $\begin{array}{r}-5.000^{*} \\
(0.57)\end{array}$ & $\begin{array}{r}-2.095^{*} \\
(0.95)\end{array}$ & $\begin{array}{r}-2.779^{*} \\
(0.57)\end{array}$ & $\begin{array}{r}-3.801^{*} \\
(0.39)\end{array}$ & $\begin{array}{r}-1.977^{*} \\
(0.25)\end{array}$ & $\begin{array}{r}-1.084^{*} \\
(0.50)\end{array}$ & $\begin{array}{r}-2.060^{*} \\
(0.29)\end{array}$ \\
\hline $\mathrm{AIC}$ & -1306.20 & -1456.58 & -5464.95 & -1957.46 & -1538.34 & -6626.42 & -660.92 & -389.85 & -925.14 \\
\hline \multicolumn{10}{|c|}{ TVP-Rotated Gumbel } \\
\hline $\bar{\omega}$ & $\begin{array}{r}0.368^{*} \\
(0.04)\end{array}$ & $\begin{array}{r}0.477^{*} \\
(0.04)\end{array}$ & $\begin{array}{r}1.162^{*} \\
(0.05)\end{array}$ & $\begin{array}{c}0.473^{*} \\
(0.04)\end{array}$ & $\begin{array}{r}0.932^{*} \\
(0.08)\end{array}$ & $\begin{array}{r}1.480^{*} \\
(0.07)\end{array}$ & $\begin{array}{r}0.448^{*} \\
(0.07)\end{array}$ & $\begin{array}{l}-0.167 \\
(0.10)\end{array}$ & $\begin{array}{l}1.381 \\
(2.72)\end{array}$ \\
\hline $\bar{\beta}$ & $\begin{array}{c}0.370^{*} \\
(0.01)\end{array}$ & $\begin{array}{r}0.340^{*} \\
(0.01)\end{array}$ & $\begin{array}{r}0.195^{*} \\
(0.01)\end{array}$ & $\begin{array}{c}0.336^{*} \\
(0.01)\end{array}$ & $\begin{array}{r}0.197^{*} \\
(0.03)\end{array}$ & $\begin{array}{r}0.160^{*} \\
(0.01)\end{array}$ & $\begin{array}{r}0.358^{*} \\
(0.02)\end{array}$ & $\begin{array}{r}0.621^{*} \\
(0.05)\end{array}$ & $\begin{array}{r}-0.111 \\
(1.37)\end{array}$ \\
\hline $\bar{\alpha}$ & $\begin{array}{r}-0.761^{*} \\
(0.12)\end{array}$ & $\begin{array}{r}-1.027^{*} \\
(0.10)\end{array}$ & $\begin{array}{r}-2.230^{*} \\
(0.29)\end{array}$ & $\begin{array}{r}-0.868^{*} \\
(0.13)\end{array}$ & $\begin{array}{r}-2.050^{*} \\
(0.14)\end{array}$ & $\begin{array}{r}-3.810^{*} \\
(0.63)\end{array}$ & $\begin{array}{r}-1.425^{*} \\
(0.18)\end{array}$ & $\begin{array}{r}-0.351^{*} \\
(0.13)\end{array}$ & $\begin{array}{l}-1.948 \\
(2.29)\end{array}$ \\
\hline $\mathrm{AIC}$ & -1553.51 & -1731.97 & -5679.02 & -2122.33 & -1756.68 & -6913.55 & -739.91 & -447.49 & -1066.97 \\
\hline
\end{tabular}

Notes. The table reports the maximum likelihood (ML) estimates for the different copula models for MSCI and the series indicated in each column. Standard error values (in brackets) and the AIC values adjusted for small-sample bias are provided for the different copula models. The minimum Akaike information criterion (AIC) value indicates the best copula fit. For the TVP-Gaussian and TVP-Student-t copulas, q in Eq. (2.14) was set to 10. An asterisk $(*)$ indicates significance at the $5 \%$ level. 
Figure 2.5 also provides information on $\Delta \mathrm{CoVaR}$ dynamics, with the value remaining relatively stable over the sampling period, but registering sudden reductions when dependence between a financial institution and the overall system reduced, thereby increasing the value of the CoVaR. In particular, there was a significant reduction in $\mathrm{CoVaR}$ and $\Delta \mathrm{CoVaR}$ for Banco de Valencia towards the end of the sampling period when this bank went bankrupt. However, this bankruptcy had little impact on the system as a whole, given the decoupling that occurred.

Table 2.9 summarizes descriptive $\mathrm{CoVaR}$ and $\Delta \mathrm{CoVaR}$ statistics for the three approaches. The average $\mathrm{CoVaR}$ values obtained with the $\mathrm{MGARCH}$ model and bivariate Student-t density and copula models were similar and much lower than for the quantile regression approach. Moreover, fluctuations in the quantile regression CoVaR values were much less than for the other two procedures, as confirmed by the differences between maximum and minimum values. We can therefore conclude that quantile regression underestimates systemic risk. Descriptive statistics for $\Delta$ CoVaR allow us to conclude that the systemically important financial institutions in the Spanish financial sector were BBVA and Santander and that systemic risk increases with the size of the financial institution.

Figure 2.5: Estimates of CoVaR (left axis) and $\Delta \mathrm{CoVaR}$ (right axis) for the MSCI Index with respect to each institution.

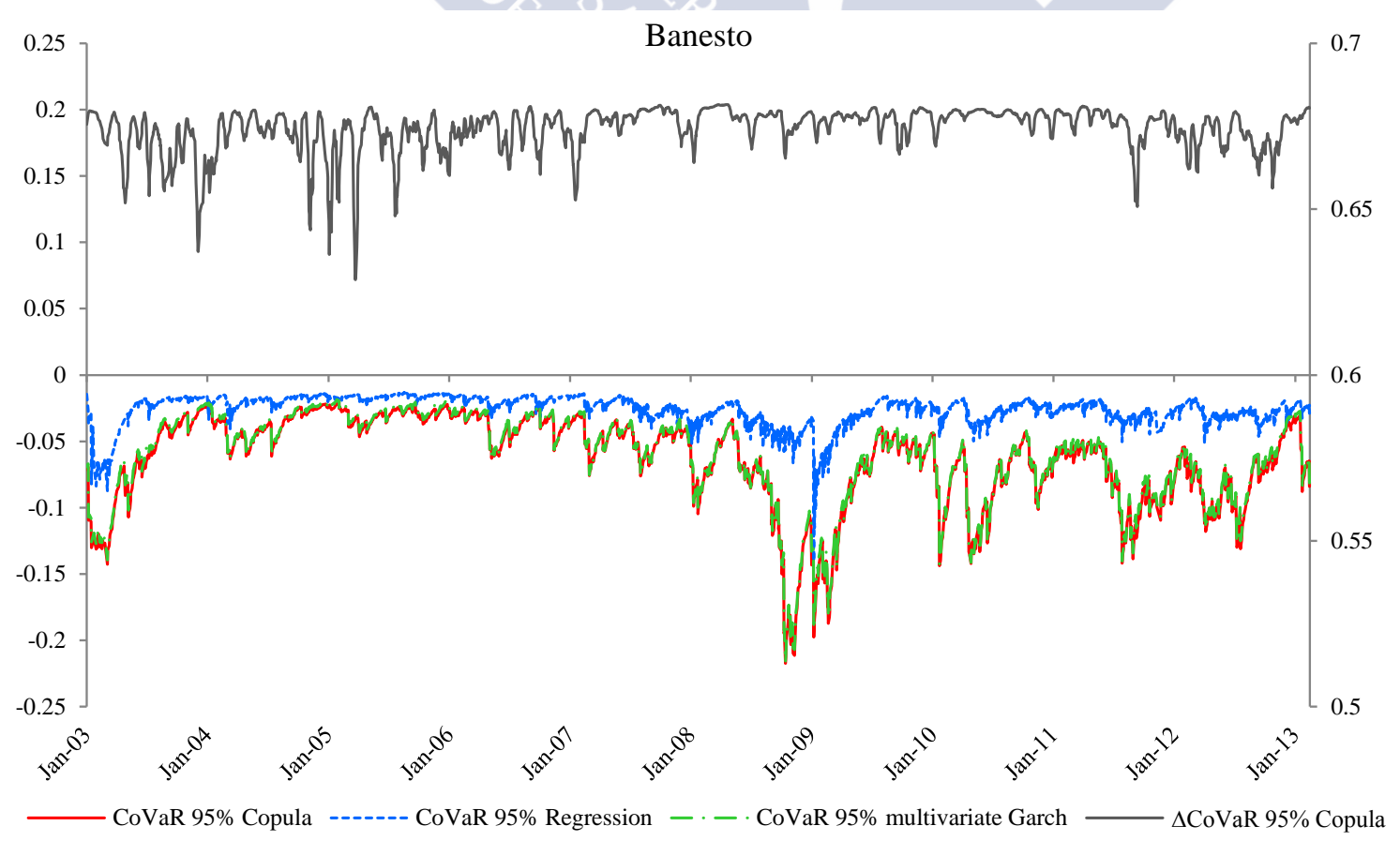


Figure 2.5: (Continued)

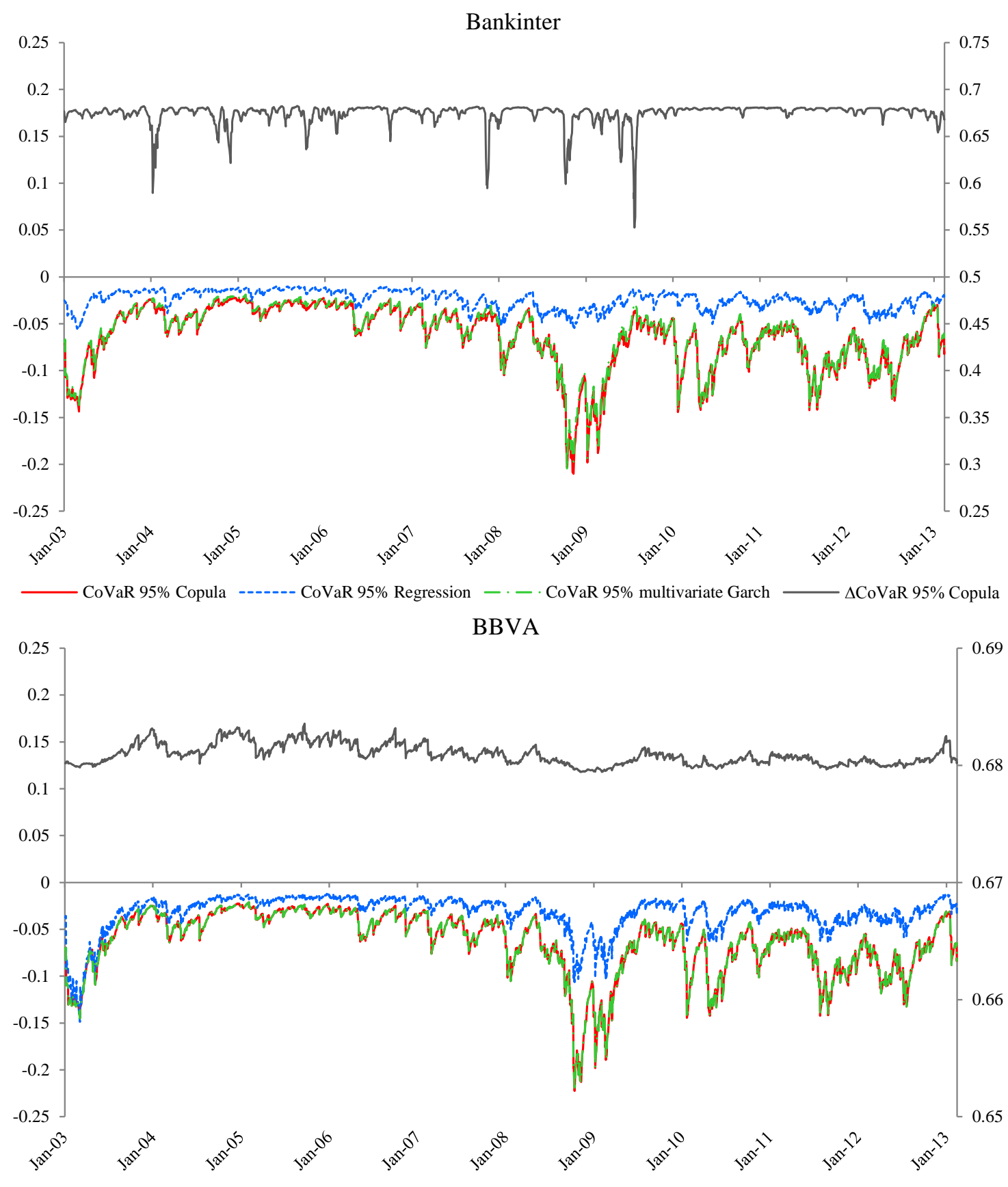

- CoVaR 95\% Copula ------ CoVaR 95\% Regression - - - CoVaR 95\% multivariate Garch $-\Delta$ CoVaR $95 \%$ Copula 
Figure 2.5: (Continued)

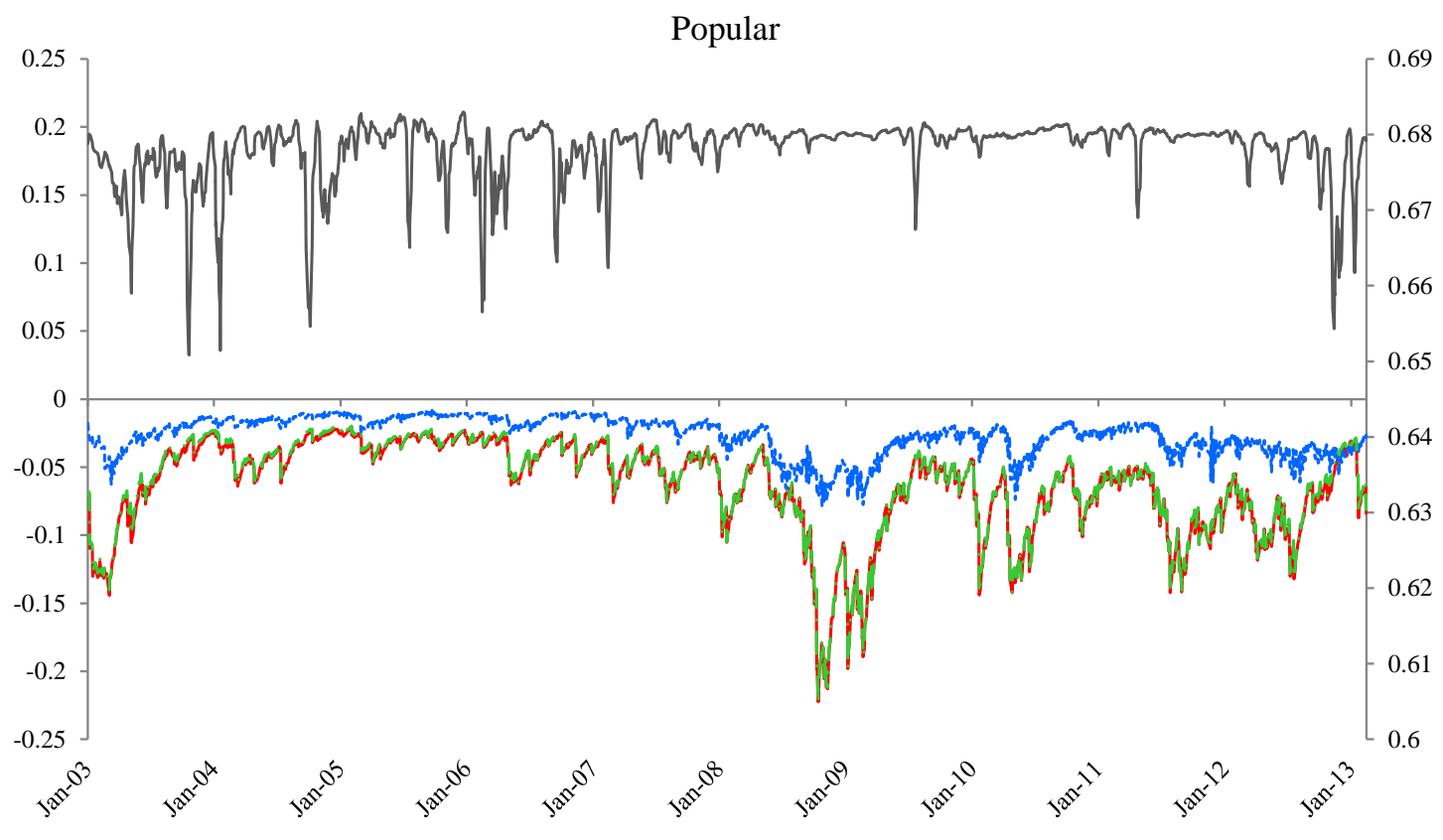

CoVaR 95\% Copula

- CoVaR 95\% Regression - - - CoVaR 95\% multivariate Garch

$\Delta$ CoVaR $95 \%$ Copula Sabadell

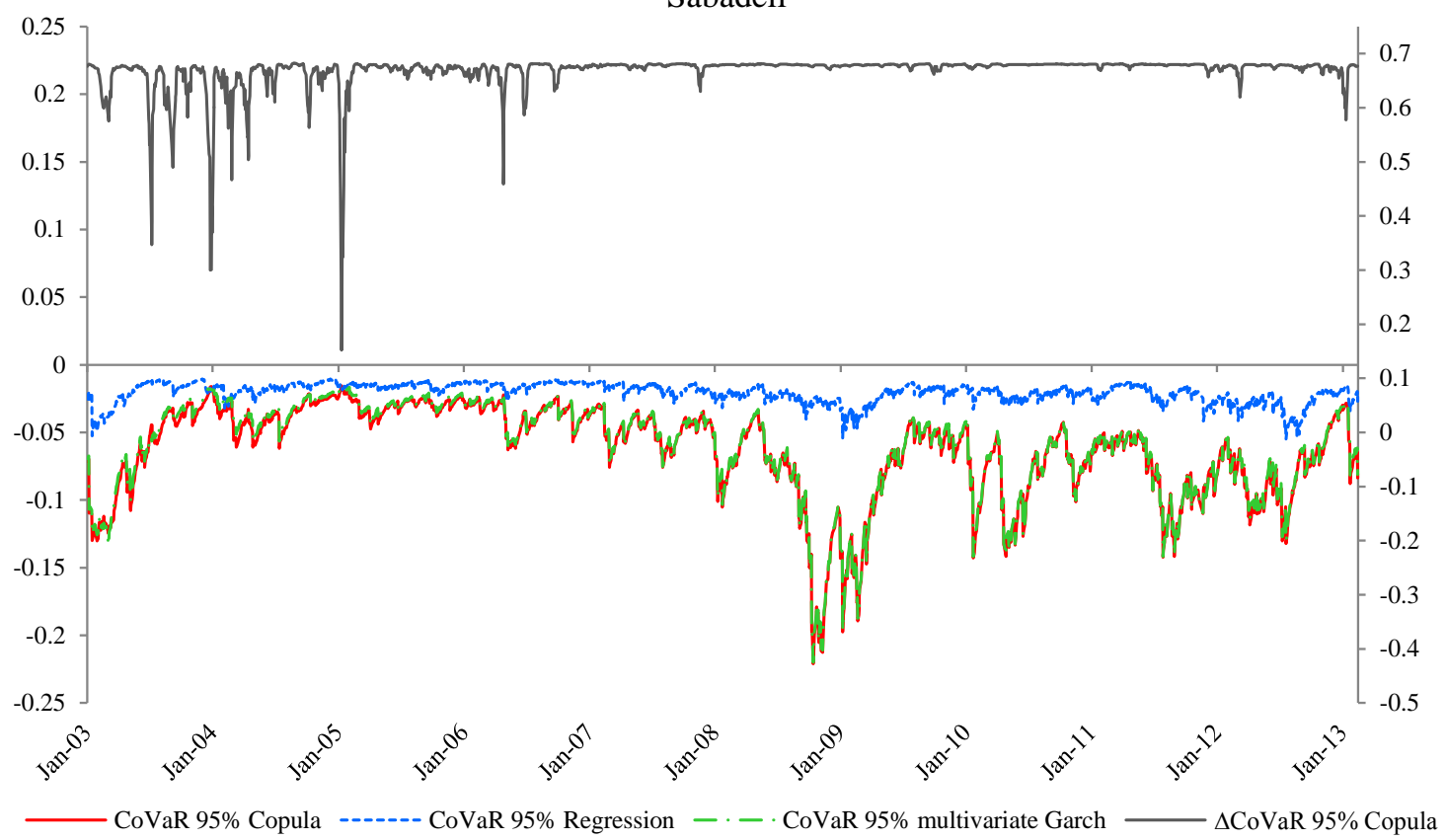


Figure 2.5: (Continued)
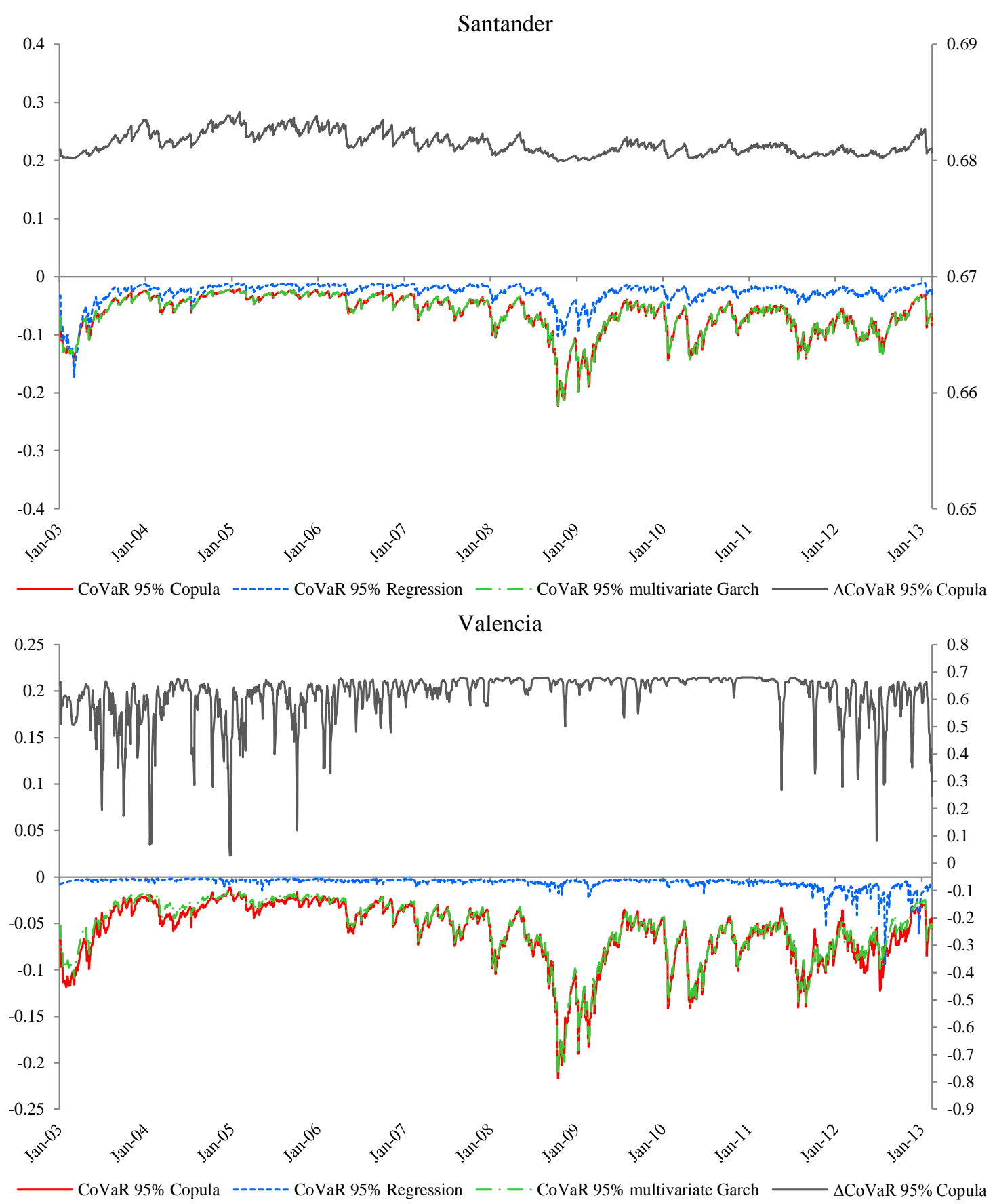
Figure 2.5: (Continued)
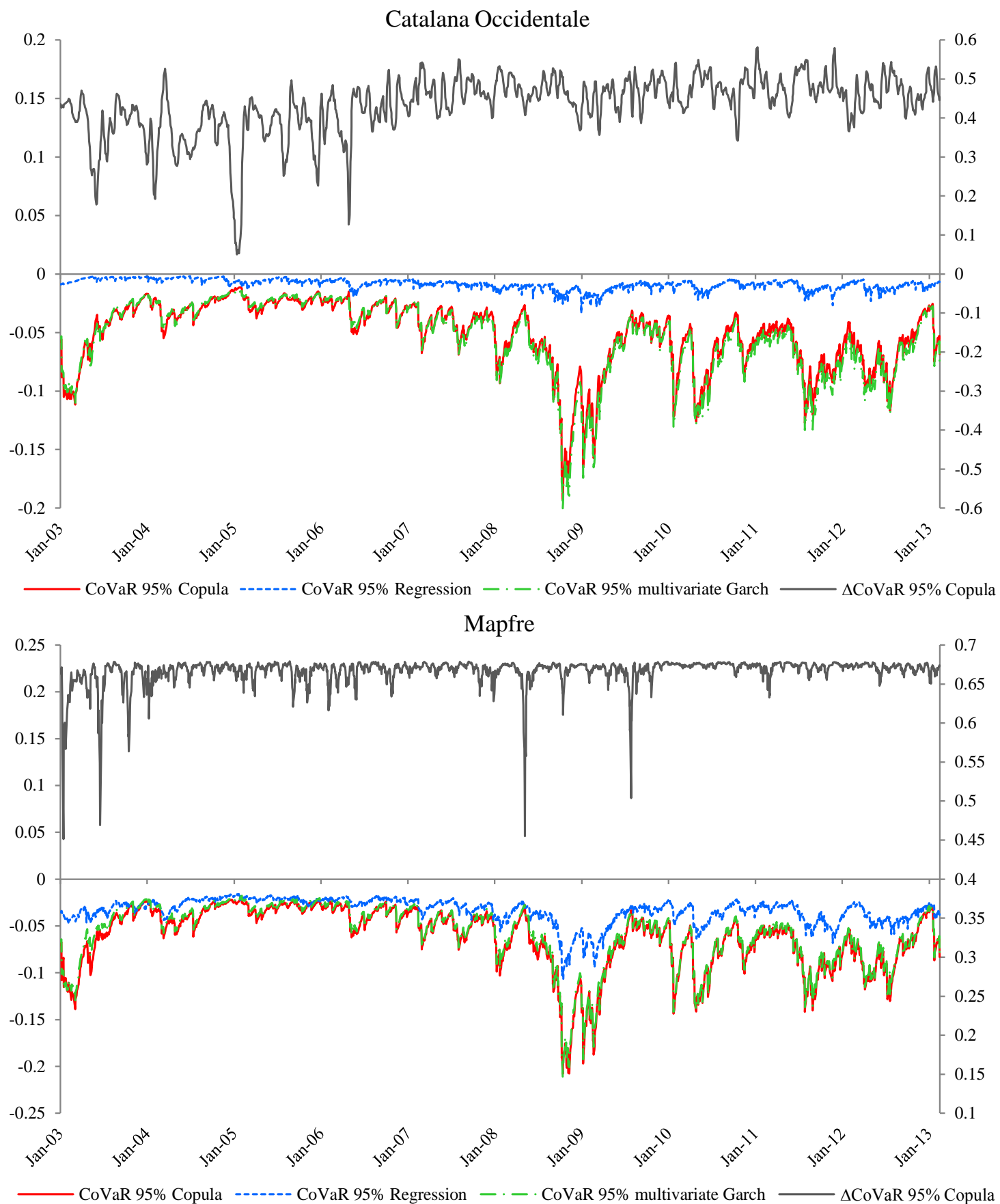
Table 2.9: Summary statistics for CoVaR-QR, CoVaR-Student-t, CoVaR-Copula and $\Delta \mathrm{CoVaR}$ - Copula for each institution with the MSCI Index.

\begin{tabular}{|c|c|c|c|c|c|}
\hline & & Mean & Std. & $\operatorname{Max}$ & Min \\
\hline \multirow{4}{*}{ Banesto } & CoVaR--QR & -0.0257 & 0.0111 & -0.0132 & -0.1405 \\
\hline & CoVaR--Student-t & -0.0605 & 0.0333 & -0.0185 & -0.2169 \\
\hline & CoVaR--Copula & -0.0626 & 0.0337 & -0.0202 & -0.2173 \\
\hline & $\Delta \mathrm{CoVaR}-\mathrm{Copula}$ & 0.6742 & 0.0066 & 0.6815 & 0.6287 \\
\hline \multirow{4}{*}{ Bankinter } & CoVaR--QR & -0.0239 & 0.0097 & -0.0101 & -0.0556 \\
\hline & CoVaR--Student-t & -0.0607 & 0.0328 & -0.0189 & -0.2056 \\
\hline & CoVaR--Copula & -0.0625 & 0.0333 & -0.0209 & -0.2102 \\
\hline & $\Delta \mathrm{CoVaR}$--Copula & 0.6745 & 0.0113 & 0.6824 & 0.5526 \\
\hline \multirow{4}{*}{ BBVA } & CoVaR--QR & -0.0312 & 0.0181 & -0.0121 & -0.1486 \\
\hline & CoVaR--Student-t & -0.0632 & 0.0339 & -0.0210 & -0.2226 \\
\hline & CoVaR--Copula & -0.0632 & 0.0338 & -0.0212 & -0.2226 \\
\hline & $\Delta$ CoVaR--Copula & 0.6809 & 0.0009 & 0.6836 & 0.6794 \\
\hline \multirow{4}{*}{ Popular } & CoVaR--QR & -0.0262 & 0.0136 & -0.0083 & -0.0783 \\
\hline & CoVaR--Student- & -0.0617 & 0.0341 & -0.0197 & -0.2214 \\
\hline & CoVaR--Copula & -0.0630 & 0.0339 & -0.0212 & -0.2225 \\
\hline & $\Delta$ CoVaR--Copula & 0.6781 & 0.0042 & 0.6830 & 0.6508 \\
\hline \multirow{4}{*}{ Sabadell } & CoVaR--QR & -0.0203 & 0.0070 & -0.0105 & -0.0550 \\
\hline & CoVaR--Student-t & -0.0607 & 0.0341 & -0.0167 & -0.2200 \\
\hline & CoVaR--Copula & -0.0624 & 0.0340 & -0.0155 & -0.2211 \\
\hline & $\Delta \mathrm{CoVaR}$--Copula & 0.6688 & 0.0352 & 0.6824 & 0.1524 \\
\hline \multirow{4}{*}{ Santander } & CoVaR--QR & -0.0265 & 0.0172 & -0.0110 & -0.1741 \\
\hline & CoVaR--Student-t & -0.0632 & 0.0339 & -0.0212 & -0.2226 \\
\hline & CoVaR --Copula & -0.0632 & 0.0338 & -0.0213 & -0.2226 \\
\hline & $\Delta \mathrm{CoVaR}$--Copula & 0.6815 & 0.0009 & 0.6842 & 0.6799 \\
\hline \multirow{4}{*}{ Valencia } & CoVaR--QR & -0.0060 & 0.0059 & -0.0018 & -0.0941 \\
\hline & CoVaR--Student-t & -0.0555 & 0.0328 & -0.0157 & -0.2141 \\
\hline & CoVaR--Copula & -0.0588 & 0.0330 & -0.0111 & -0.2169 \\
\hline & $\Delta$ CoVaR--Copula & 0.6172 & 0.0860 & 0.6810 & 0.0283 \\
\hline \multirow{4}{*}{ Catalana } & CoVaR--QR & -0.0087 & 0.0044 & -0.0019 & -0.0335 \\
\hline & CoVaR--Student-t & -0.0542 & 0.0318 & -0.0145 & -0.2004 \\
\hline & CoVaR--Copula & -0.0517 & 0.0293 & -0.0115 & -0.1929 \\
\hline & $\Delta \mathrm{CoVaR}-\mathrm{Copula}$ & 0.4332 & 0.0755 & 0.5810 & 0.0504 \\
\hline \multirow{4}{*}{ Mapfre } & CoVaR--QR & -0.0333 & 0.0132 & -0.0161 & -0.1072 \\
\hline & CoVaR--Student-t & -0.0587 & 0.0328 & -0.0177 & -0.2127 \\
\hline & CoVaR--Copula & -0.0619 & 0.0332 & -0.0204 & -0.2082 \\
\hline & $\Delta$ CoVaR--Copula & 0.6669 & 0.0172 & 0.6791 & 0.4513 \\
\hline
\end{tabular}

Notes. The table reports descriptive statistic for the $\mathrm{CoVaR}$ and $\Delta \mathrm{CoVaR}$ in percentages for each institution at the $95 \%$ confidence level, given a VaR level at $95 \%$ for the MSCI Index and using quantile regression (QR), the multivariate generalized autoregressive conditional heteroskedasticitydynamic conditional correlation (MGARCH-DCC) model with a bivariate Student-t distribution and the best copula fits. Std., Max and Min denote standard deviation, maximum and minimum, respectively. 
Finally, we considered the relationship between the VaR and CoVaR, as represented in Figure 2.6. Our results reveal a weak relationship between the $\Delta$ CoVaR and VaR, calculated using the quantile regression, MGARCH and copula approaches - a result that is consistent with that reported by Girardi and Ergün (2003). This suggests that the VaR is incapable of determining capital requirements for financial institutions that would overcome market risk.

Figure 2.6: Scatter plot for $\Delta \mathrm{CoVaR}$ vs VaR.

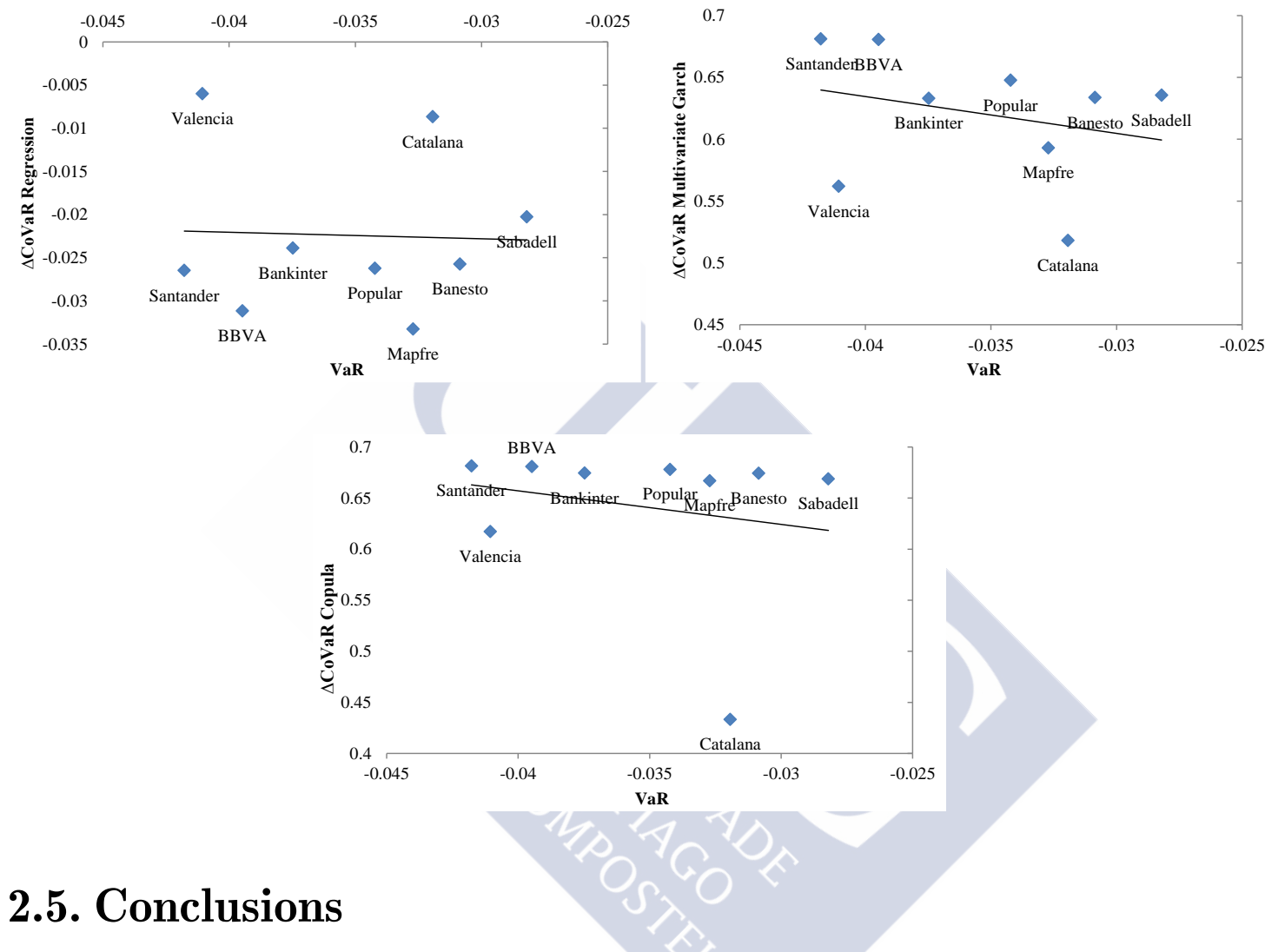

Recent financial and debt crises have raised public and regulatory concerns regarding the systemic risk impact of failed or failing financial institutions. Accurate assessment of systemic risk is crucial for effective regulation of risk and to ameliorate the impact of financial crises on the performance of financial systems. We studied the systemic risk of Spanish financial institutions around the time of the recent global financial crisis and the European sovereign debt crisis. To that end, we quantified the CoVaR for several listed Spanish financial institutions for the period January 2003 to February 2013 and characterized dynamic behaviour using three different approaches: quantile regression, MGARCH model and copulas.

We found that the CoVaR measure of systemic risk displayed trends that were consistent for all the institutions throughout the sampling period, taking lower 
values around the periods of the crises, especially around the recent global financial crisis. We also observed that the CoVaR computed using quantile regression was not able to capture the dynamics of systemic risk, as it lacked the flexibility to adapt to crisis periods when volatility was high; consequently it underestimated systemic risk. The MGARCH and copula approaches were more flexible in identifying crisis periods with significant reductions in the $\mathrm{CoVaR}$ value. We also found that the copula approach reflected greater systemic risk than the MGARCH approach; this evidence is consistent with the fact that tail dependence is better captured by a copula than by a parametric multivariate distribution function. The risk contribution of the financial institutions, as evaluated using $\Delta \mathrm{CoVaR}$, amounted to around $65 \%$.

Overall, our measurements of systemic risk allow us to conclude that systemic risk increased during the financial crisis but not with the same intensity for all the studied institutions; it mainly affected larger institutions that assumed increasingly systemic importance and were considered to be too interconnected to fail. These results have implications for regulatory policy aimed at determining optimal capital requirements and cyclical changes in value, mainly for large financial entities. 


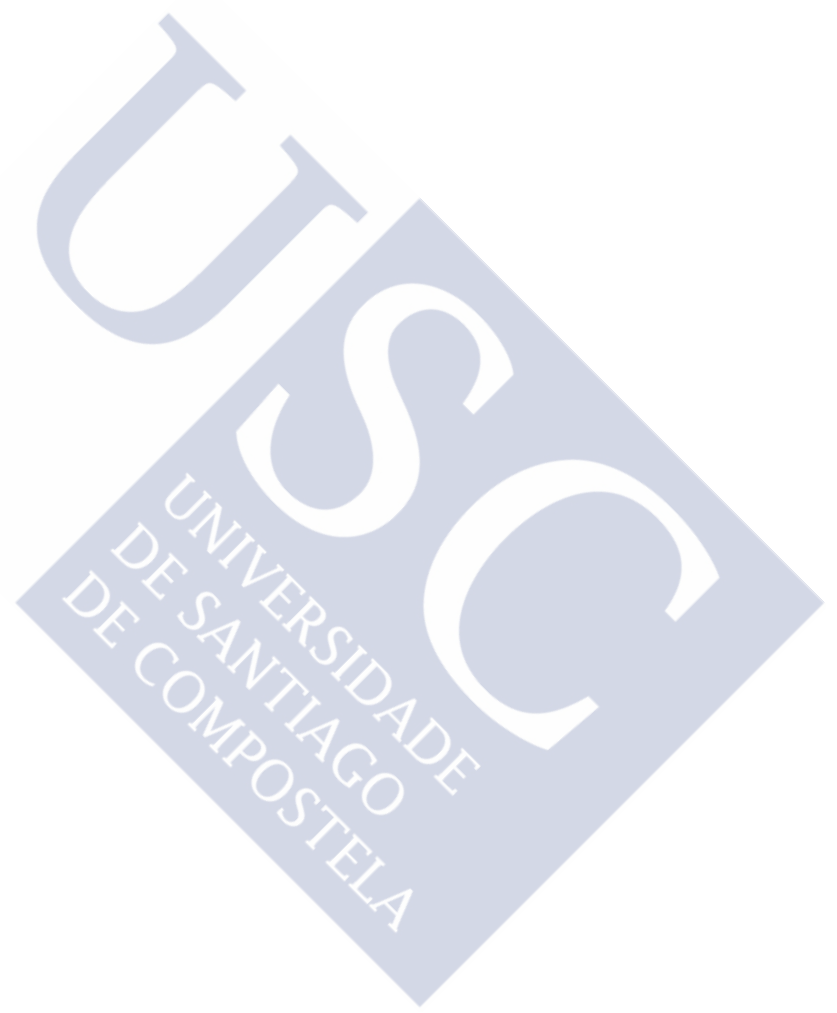




\section{Chapter 3}

\section{Systemic risk in European sovereign debt markets: A CoVaR-copula approach}

\subsection{Introduction}

The recent sovereign debt crisis in Europe has raised concerns about the fragility of the debt markets and the potential systemic risk effects of a sovereign default in the euro area. Measuring systemic risk among debt markets is crucial to an assessment of how the deteriorated financial position of a sovereign market can impair the performance of other sovereign debt markets. In this chapter, we quantify systemic risk in the European sovereign debt markets before and after the onset of the European sovereign debt crisis by computing the conditional value-at-risk (CoVaR) through copulas, providing thus quantitative evidence regarding how systemic risk has changed as a result of the debt crisis.

One strand of the burgeoning literature on the European sovereign debt crisis examines co-movements and drivers of fluctuations in government bond spreads such as credit risk, exchange rate movements, specific news, rating changes or the probability of some countries exiting the euro area (see, e.g., Manganelli and Wolswijk, 2009; Haugh et al., 2009; Schuknecht et al., 2010; Borgy et al., 2011; de Santis, 2013; Beestma et al. 2013; Favero, 2013). Another strand of this literature is concerned with the spillover effects of the European debt crisis on the financial 
sector. De Bruyckere et al. (2013) studied contagion between bank and sovereign default risk in Europe through asset, collateral and rating channels. Bhanot et al. (2014) investigated the impact of changes in Greek sovereign yield spreads on stock returns in the financial sector. Similarly, Mink and De Haan (2013) analysed the impact of highly volatile Greek bonds on European bank stock prices in 2010. Alter and Schuler (2012) examined the relationship between sovereign default risk and domestic banks. In addition, using credit default swaps, other studies examined sovereign risk contagion among eurozone countries (see, e.g., Missio and Watzka, 2011; Arezki et al., 2011; Alter and Beyer, 2012; Caporin et al., 2013). However, even though systemic risk is an important dimension of contagion that enables to quantify the impact of extreme downward movement in one market on other markets, no study has yet examined systemic risk in European sovereign debt markets and how this risk has changed with the onset of the recent European sovereign debt crisis. This chapter attempts to fill this gap, contributing, in particular, to the existing literature in two ways.

First, we characterize the CoVaR systemic risk measure - as proposed by Adrian and Brunnermeier (2011) and generalized by Girardi and Ergün (2013) - in terms of copulas. CoVaR captures possible risk spillovers between markets by providing information on the value-at-risk (VaR) of a market, conditional on the fact that another market is in financial distress. Using copulas, the value of the CoVaR can be obtained in a two-step procedure. Given the cumulative probability of the VaR of the market in financial distress and the confidence level for the CoVaR, we can compute the cumulative probability for the CoVaR from a copula function. We then can invert the marginal distribution function for this cumulative probability in order to obtain the value of the CoVaR. From a computational point of view, this approach is more tractable than other parametric approaches; it is also more flexible, given that copula functions, by providing a measure of both average dependence and upper and lower tail dependence (joint extreme movements), enable the dependence structure of stochastic variables to be fully described. This information is crucial to determining the $\mathrm{VaR}$ of one variable, which is conditional on the fact that another variable takes values below or equal to its own VaR. In fact, (lower) tail dependence of a copula function naturally provides this information, but at the limit.

Second, for a sample of sovereign bond benchmark price indices for France, Germany and the Netherlands, for GIIPS economies (Greece, Ireland, Italy, Portugal and Spain) and for an overall sovereign bond price index for the European Economic 
and Monetary Union (EMU), for the period January 2000 to October 2012, we provide evidence of strong co-movement between European debt markets and the EMU index before the onset of the European debt crisis. All sovereign debt markets shared a similar trend in systemic risk, which was very similar in size across markets. However, with the onset of the European sovereign debt crisis, we find evidence of the decoupling of debt markets in such a way that GIIPS markets negatively correlate, on average, with the EMU index returns, displaying, in general, market independence at the tails. As a result, systemic risk for the GIIPS markets, with the exception of Spain, was reduced, whereas systemic risk for the non-crisis countries experienced a significant upsurge as a result of a high degree of comovement with the EMU index. Finally, we examined the systemic risk impact of the Greek debt market on other European debt markets, finding that before the Greek debt crisis, Greek systemic risk was relatively low and different across countries. However, after crisis onset, Greek systemic risk increased for the countries in crisis, especially for Portugal, where systemic risk tripled overall. For countries not in crisis the systemic impact of the Greek debt crisis was less, given that the debt markets of these countries decoupled from the Greek market.

The remainder of the chapter is laid out as follows: in Section 3.2 we outline the copula approach to the CoVaR, in Section 3.3 we present data and in Section 3.4 we discuss the results. Finally, Section 3.5 concludes the chapter.

\subsection{Methodology}

With the aim of quantifying systemic risk between assets, institutions or markets, different systemic risk measures have been proposed in the literature (see, e.g., Huang et al., 2009; Segoviano and Goodhart, 2009; Acharya et al., 2010; Allen et al., 2010; Zhou, 2010, Adrian and Brunnermeier, 2011; Brownlees and Engle, 2011; Billio et al., 2012; Girardi and Ergün, 2013; Gravelle and Li, 2013). Given that VaR is arguably the most widely employed risk measure by investors, financial institutions and regulators, in this study we evaluate systemic risk between European debt markets using the CoVaR measure proposed by Adrian and Brunnermeier (2011) and generalized by Girardi and Ergün (2013). 


\subsubsection{CoVaR and copulas}

The CoVaR for the European debt market is the VaR for the European debt market as a whole, conditional on the fact that a given debt market is in financial distress. Let $\mathrm{R}_{\mathrm{t}}^{\mathrm{d}}$ be the returns for the debt market as a whole and let $\mathrm{R}_{\mathrm{t}}^{\mathrm{j}}$ be the returns for debt market $j$. The CoVaR, formally defined as the $\beta$-quantile of the conditional distribution of $\mathrm{R}_{\mathrm{t}}^{\mathrm{d}}$, is as follows:

$$
\operatorname{Pr}\left(R_{t}^{\mathrm{d}} \leq \operatorname{CoVaR}_{\beta, \mathrm{t}}^{\mathrm{d} \mid \mathrm{j}} \mid \mathrm{R}_{\mathrm{t}}^{\mathrm{j}} \leq \operatorname{VaR}_{\alpha, \mathrm{t}}^{\mathrm{j}}\right)=\beta
$$

where $\mathrm{VaR}_{\alpha, t}^{\mathrm{j}}$ is the $\mathrm{VaR}$ for debt market $\mathrm{j}$, measuring the maximum loss that debt market $\mathrm{j}$ may experience for a confidence level $1-\alpha$ and a specific time horizon, that is, the $\alpha$-quantile of the return distribution for the debt market $j$ : $\operatorname{Pr}\left(R_{t}^{j} \leq \operatorname{VaR}_{\alpha, t}^{j}\right)=\alpha$. Therefore, computing the CoVaR consists of determining the quantile of a conditional distribution, or, alternatively, of an unconditional bivariate distribution if we express Eq. (3.1) as:

$$
\frac{\operatorname{Pr}\left(R_{t}^{\mathrm{d}} \leq \mathrm{CoVaR}_{\beta, \mathrm{t}}^{\mathrm{d} \mid \mathrm{j}}, \mathrm{R}_{\mathrm{t}}^{\mathrm{j}} \leq \mathrm{VaR}_{\alpha, \mathrm{t}}^{\mathrm{j}}\right)}{\operatorname{Pr}\left(\mathrm{R}_{\mathrm{t}}^{\mathrm{j}} \leq \mathrm{VaR}_{\alpha, \mathrm{t}}^{\mathrm{j}}\right)}=\beta .
$$

Given that $\operatorname{Pr}\left(\mathrm{R}_{\mathrm{t}}^{\mathrm{j}} \leq \mathrm{VaR}_{\alpha, \mathrm{t}}^{\mathrm{j}}\right)=\alpha$, the CoVaR in Eq. (3.2) can be expressed as:

$$
\operatorname{Pr}\left(\mathrm{R}_{\mathrm{t}}^{\mathrm{d}} \leq \mathrm{CoVaR}_{\beta, \mathrm{t}}^{\mathrm{d} \mid \mathrm{j}}, \mathrm{R}_{\mathrm{t}}^{\mathrm{j}} \leq \operatorname{VaR}_{\alpha, \mathrm{t}}^{\mathrm{j}}\right)=\alpha \beta .
$$

Girardi and Ergün (2013) proposed to compute for the CoVaR in Eq. (3.3) by numerically solving a double integral:

$$
\int_{-\infty}^{\operatorname{CoVaR} \mathrm{R}_{\beta, \mathrm{t}}^{\mathrm{d} j}} \int_{-\infty}^{\mathrm{VaR}}{ }_{\alpha, \mathrm{t}}^{\mathrm{j}} f_{\mathrm{t}}\left(\mathrm{R}_{\mathrm{t}}^{\mathrm{d}}, \mathrm{R}_{\mathrm{t}}^{\mathrm{j}}\right) d \mathrm{R}_{\mathrm{t}}^{\mathrm{d}} d \mathrm{R}_{\mathrm{t}}^{\mathrm{j}}=\alpha \beta
$$

for given levels of $\alpha, \beta$ and $\operatorname{VaR}_{\alpha, t}^{\mathrm{j}}$; and where $f_{t}\left(\mathrm{R}_{\mathrm{t}}^{\mathrm{d}}, \mathrm{R}_{\mathrm{t}}^{\mathrm{j}}\right)$ is the bivariate density of $\mathrm{R}_{\mathrm{t}}^{\mathrm{d}}$ and $\mathrm{R}_{\mathrm{t}}^{\mathrm{j}}$.

In this chapter we propose to compute the CoVaR through copulas. ${ }^{5}$ Note that Eq. (3.3) can be expressed in terms of the joint distribution function of $R_{t}^{d}$ and $R_{t}^{j}$, $\mathrm{F}_{\mathrm{R}_{\mathrm{t}}^{\mathrm{d}}, \mathrm{R}_{\mathrm{t}}^{\mathrm{j}}}$, as:

\footnotetext{
${ }^{5}$ For further analysis on copulas, see Joe (1997) and Nelsen (2006). An overview of copula applications to finance can be found in Cherubini et al. (2004). Mainik and Schaanning (2012) provide the first representation of the $\mathrm{CoVaR}$ in terms of copulas.
} 


$$
\mathrm{F}_{\mathrm{R}_{\mathrm{t}}^{\mathrm{d}}, \mathrm{R}_{\mathrm{t}}^{\mathrm{j}}}\left(\mathrm{CoVaR}_{\beta, \mathrm{t}}^{\mathrm{d} \mid \mathrm{j}}, \operatorname{VaR}_{\alpha, \mathrm{t}}^{\mathrm{j}}\right)=\alpha \beta
$$

and that, according to Sklar's (1959) theorem, the joint distribution function of two continuous variables can be expressed in terms of a copula function. Hence, Eq. (3.5) can be written as:

$$
\mathrm{C}(\mathrm{u}, \mathrm{v})=\alpha \beta,
$$

where $\mathrm{C}(\cdot, \cdot)$ is a copula function, $\mathrm{u}=\mathrm{F}_{\mathrm{R}_{\mathrm{t}}^{\mathrm{d}}}\left(\mathrm{CoVaR}_{\beta, \mathrm{t}}^{\mathrm{d} \mid \mathrm{j}}\right)$ and $\mathrm{v}=\mathrm{F}_{\mathrm{R}_{\mathrm{t}}^{\mathrm{j}}}\left(\mathrm{VaR}_{\alpha, \mathrm{t}}^{\mathrm{j}}\right)$ and where $\mathrm{F}_{\mathrm{R}_{\mathrm{t}}^{\mathrm{d}}}$ and $\mathrm{F}_{\mathrm{R}_{\mathrm{t}}^{\mathrm{j}}}$ are the marginal distribution functions of $\mathrm{R}_{\mathrm{t}}^{\mathrm{d}}$ and $\mathrm{R}_{\mathrm{t}}^{\mathrm{j}}$, respectively. Given its copula representation in Eq. (3.6), the CoVaR can be computed from that equation through copulas in a two-step procedure:

1) We obtain the value of $u=F_{R_{t}^{d}}\left(\operatorname{CoVaR}_{\beta, t}^{d \mid j}\right)$. Since $C(u, v)=\alpha \beta$, where $\alpha, \beta$ and $v$ are given (note that $\mathrm{v}=\alpha$ ), from the copula function specification we can solve to determine the value of $\mathrm{u}$.

2) Taking $u$, we can obtain the CoVaR value as the quantile of the distribution of $\mathrm{R}_{\mathrm{t}}^{\mathrm{d}}$, with a cumulative probability equal to $\mathrm{u}$, by inverting the marginal distribution function of $\mathrm{R}_{\mathrm{t}}^{\mathrm{d}}$ : $\operatorname{CoVaR}_{\beta, \mathrm{t}}^{\mathrm{d} \mid \mathrm{j}}=\mathrm{F}_{\mathrm{R}_{\mathrm{d}}^{\mathrm{d}}}^{-1}(\mathrm{u})$.

Computing the CoVaR through copulas has two main advantages. First, since copulas allow separate modelling of the marginals and dependence structures, they offer great flexibility in modelling marginals. This flexibility is crucial for the computation of $\mathrm{VaR}$ and the modelling of dependence structures with different tail dependence characteristics, such as the tail independence and symmetric or asymmetric tail dependence that is especially relevant for the computation of the CoVaR measure. Furthermore, copulas are useful when the joint distribution function is not elliptical, when the traditional dependence measure given by the linear correlation coefficient is insufficient to describe the dependence structure (see Embrechts et al. 2003). This is especially relevant when the bivariate Gaussian or Student-t distributions (both widely used in multivariate generalized autoregressive conditional heteroskedasticity (GARCH) models) do not adequately represent the joint distribution function of the data. Second, computation of the CoVaR using copulas is computationally more tractable than obtaining the value of $\mathrm{CoVaR}$ using Eq. (3.4), as the equation requires numerical resolution of a double integral and VaR computation for the financially distressed market. Note that, using a copula 
characterization of the CoVaR, we only need information on the cumulative probability for the VaR and not the value of the VaR itself.

Adrian and Brunnermeier (2011) and Girardi and Ergün (2013) define the systemic risk contribution of a particular market $\mathrm{j}$ as the delta $\mathrm{CoVaR}(\Delta \mathrm{CoVaR})$, which is the difference between the VaR of the European debt market as a whole conditional on the distressed state of market $\mathrm{j}\left(\mathrm{R}_{\mathrm{t}}^{\mathrm{j}} \leq \mathrm{VaR}_{\alpha, \mathrm{t}}^{\mathrm{j}}\right)$ and the $\mathrm{VaR}$ of the European debt market as a whole conditional on the benchmark state of market $j$, considering it as the median of the return distribution of market $j$, or, alternatively, the $\mathrm{VaR}$ for an $\alpha=0.5$. The systemic risk contribution of market $\mathrm{j}$ is thus defined as:

$$
\Delta \operatorname{CoVaR}_{\mathrm{t}}^{\mathrm{d} \mid \mathrm{j}}=\operatorname{CoVaR}_{\beta, \mathrm{t}}^{\mathrm{d} \mid \mathrm{j}}-\operatorname{CoVaR}_{\beta, \mathrm{t}}^{\mathrm{d} \mid \mathrm{j}, \alpha=0.5} / \operatorname{CoVaR}_{\beta, \mathrm{t}}^{\mathrm{d} \mid \mathrm{j}, \alpha=0.5}
$$

\subsubsection{Marginal distribution and copula models}

The marginal models and copula specifications we used to compute the CoVaR measure for the European sovereign debt markets are described as follows.

For the marginal models, following Bhanot et al. (2014), we consider that the conditional mean of the market returns for a European debt market $\mathrm{j}$ are given as a function of common and specific factors. Common factors are given by interbank interest rate changes, measured by changes in the Euribor rate (E), with a dummy variable (CRISIS) denoting periods before and after the onset of the European sovereign debt crisis as 0 and 1, respectively. Included as specific factors are the stock market index returns $\left(r_{j, t}\right)$ and market volatility $\left(\operatorname{vol}_{j, t}\right)$ for each country and lagged values for the debt market returns. Thus, the marginal model for market $\mathrm{j}$ is specified as:

$$
\mathrm{R}_{\mathrm{t}}^{\mathrm{j}}=\phi_{\mathrm{j}, 0}+\sum_{\mathrm{h}=1}^{\mathrm{p}} \phi_{\mathrm{j}, \mathrm{h}} \mathrm{R}_{\mathrm{t}-\mathrm{h}}^{\mathrm{j}}+\gamma_{\mathrm{j}, \mathrm{t}} \mathrm{r}_{\mathrm{j}}+\varphi_{\mathrm{j}, \mathrm{vol}}+\eta_{\mathrm{j}} \mathrm{E}_{\mathrm{t}}+\psi_{\mathrm{j}} \operatorname{Crisis}_{\mathrm{t}}+\varepsilon_{\mathrm{j}, \mathrm{t}},
$$

with $\varepsilon_{\mathrm{j}, \mathrm{t}}=\sigma_{\mathrm{j}, \mathrm{t}} \mathrm{z}_{\mathrm{j}, \mathrm{t}}$, where $\sigma_{\mathrm{j}, \mathrm{t}}^{2}$ is the conditional variance, given by a threshold generalized autoregressive conditional heteroskedasticity (TGARCH) specification (Zakoian, 1994; Glosten et al., 1993):

$$
\sigma_{\mathrm{j}, \mathrm{t}}^{2}=\omega_{\mathrm{j}}+\sum_{\mathrm{k}=1}^{\mathrm{r}} \mathrm{b}_{\mathrm{j}, \mathrm{k}} \sigma_{\mathrm{j}, \mathrm{t}-\mathrm{k}}^{2}+\sum_{\mathrm{h}=1}^{\mathrm{m}} \mathrm{a}_{\mathrm{j}, \mathrm{h}} \varepsilon_{\mathrm{j}, \mathrm{t}-\mathrm{h}}^{2}+\sum_{\mathrm{h}=1}^{\mathrm{m}} \lambda_{\mathrm{j}, \mathrm{h}} \varepsilon_{\mathrm{j}, \mathrm{t}-\mathrm{h}}+\mathrm{d}_{\mathrm{j}} \operatorname{Crisis}_{\mathrm{t}}
$$


where $\omega_{\mathrm{j}}$ is a constant, $\sigma_{\mathrm{j}, \mathrm{t}-\mathrm{k}}^{2}$ is the GARCH component and $\varepsilon_{\mathrm{j}, \mathrm{t}-\mathrm{h}}$ is the autoregressive conditional heteroskedasticity $(\mathrm{ARCH})$ component and where $\lambda$ captures leverage effects. If $\lambda<0$, then the future conditional variance will proportionally increase more following a negative shock than following a positive shock of the same magnitude. The crisis dummy variable is included in the volatility specification to take into account the potential effect of the sovereign debt crisis on volatility. $\mathrm{z}_{\mathrm{j}, \mathrm{t}}$ is a i.i.d. random variable with zero mean and unit variance that follows a Hansen's (1994) skewed-t density distribution. It is given by:

$$
f\left(z_{j, t} ; v, \eta\right)=\left\{\begin{array}{l}
b c\left(1+\frac{1}{v-2}\left(\frac{b_{j, t}+a}{1-\eta}\right)^{2}\right)^{-(v+1) / 2} z_{j, t}<-a / b \\
b c\left(1+\frac{1}{v-2}\left(\frac{b z_{j, t}+a}{1+\eta}\right)^{2}\right)^{-(v+1) / 2} z_{j, t} \geq-a / b
\end{array},\right.
$$

where $v$ and $\eta$ are the degrees of freedom parameter $(2<v<\infty)$ and the symmetric parameter $(-1<\eta<1)$, respectively. The constants $\mathrm{a}, \mathrm{b}$ and $\mathrm{c}$ are given by $\mathrm{a}=4 \eta \mathrm{c}\left(\frac{\mathrm{v}-2}{\mathrm{v}-1}\right), \quad \mathrm{b}^{2}=1+3 \eta^{2}-\mathrm{a}^{2}, \quad \mathrm{c}=\Gamma\left(\frac{\mathrm{v}+1}{2}\right) / \sqrt{\pi(\mathrm{v}-2)} \Gamma\left(\frac{\mathrm{v}}{2}\right)$. This distribution converges to the standard Gaussian as $\eta=0$ and $v \rightarrow \infty$, and to the symmetric Student- $t$ distribution as $\eta=0$ and $v$ is finite.

We used seven different copula specifications to capture different characteristics of dependence, as follows:

1) The bivariate Gaussian is the most commonly employed distribution in the finance literature. It is defined by $\mathrm{C}_{\mathrm{N}}(\mathrm{u}, \mathrm{v} ; \rho)=\Phi\left(\Phi^{-1}(\mathrm{u}), \Phi^{-1}(\mathrm{v})\right)$, where $\Phi$ is the bivariate standard normal cumulative distribution function with correlation $\rho$ between $\mathrm{X}$ and $\mathrm{Y}$ and where $\Phi^{-1}(\mathrm{u})$ and $\Phi^{-1}(\mathrm{v})$ are standard normal quantile functions. It has no tail dependence.

2) The Student-t is useful for capturing symmetric tail dependence. It is given by $\mathrm{C}_{\mathrm{ST}}(\mathrm{u}, \mathrm{v} ; \rho, \mathrm{v})=\mathrm{T}\left(\mathrm{t}_{\mathrm{v}}^{-1}(\mathrm{u}), \mathrm{t}_{\mathrm{v}}^{-1}(\mathrm{v})\right)$, where $\mathrm{T}$ is the bivariate Student-t cumulative distribution function with degree-of-freedom parameter $v$ and correlation $\rho$ and where $\mathrm{t}_{v}^{-1}(\mathrm{u})$ and $\mathrm{t}_{\mathrm{v}}^{-1}(\mathrm{v})$ are the quantile functions of the univariate Student-t distribution with degree-of-freedom parameter $v$. 
3) The Gumbel copula is asymmetric and displays upper tail dependence and lower tail independence. It is given by $\mathrm{C}_{\mathrm{G}}(\mathrm{u}, \mathrm{v} ; \delta)=\exp \left(-\left((-\log \mathrm{u})^{\delta}+(-\log \mathrm{v})^{\delta}\right)^{1 / \delta}\right)$. Note that the two variables are independent when $\delta=1$.

4) The rotated Gumbel copula displays upper tail independence and lower tail dependence. It is given by $\mathrm{C}_{\mathrm{RG}}(\mathrm{u}, \mathrm{v} ; \delta)=\mathrm{u}+\mathrm{v}-1+\mathrm{C}_{\mathrm{G}}(1-\mathrm{u}, 1-\mathrm{v} ; \delta)$.

5) The BB7 copula allows for different degrees of upper and lower tail dependence. It is defined as:

$$
\mathrm{C}_{\mathrm{BB} 7}(\mathrm{u}, \mathrm{v} ; \delta, \theta)=1-\left(1-\left[\left(1-(1-\mathrm{u})^{\theta}\right)^{-\delta}+\left(1-(1-\mathrm{v})^{\theta}\right)^{-\delta}-1\right]^{-1 / \delta}\right)^{1 / \theta},
$$

with, $\theta \geq 1 \delta>0$.

6) The Plackett copula is a symmetric copula which, like the Gaussian copula, exhibits tail independence, even though the dependence for large joint realizations is less than for the Gaussian copula. It is given by:

$$
\mathrm{C}_{\mathrm{P}}(\mathrm{u}, \mathrm{v} ; \theta)=\frac{1}{2(\theta-1)}(1+(\theta-1)(\mathrm{u}+\mathrm{v}))-\sqrt{(1+(\theta-1)(\mathrm{u}+\mathrm{v}))^{2}-4 \theta(\theta-1) \mathrm{uv}} .
$$

7) The Clayton-Gumbel or BB1 copula allows for asymmetric tail dependence. It is specified as

$$
\mathrm{C}_{\mathrm{CG}}(\mathrm{u}, \mathrm{v} ; \delta, \theta)=\left\{\left[\left(\mathrm{u}^{-\theta}-1\right)^{\delta}+\left(\mathrm{v}^{-\theta}-1\right)^{\delta}\right]^{1 / \delta}+1\right\}^{-1 / \theta},
$$

with $\theta>0, \delta \geq 1$.

In addition, we considered possible time-varying dependence by allowing the parameters of some copulas to vary according to a specific evolution equation. For the Gaussian and the Student-t copulas, we specified the linear dependence parameter $\rho_{\mathrm{t}}$ so that it evolves according to a model with 1 autoregressive term and q moving-average terms, that is, an $\operatorname{ARMA}(1, \mathrm{q})$-type process (Patton, 2006):

$$
\rho_{\mathrm{t}}=\Lambda\left(\psi_{0}+\psi_{1} \rho_{\mathrm{t}-1}+\psi_{2} \frac{1}{\mathrm{q}} \sum_{\mathrm{j}=1}^{\mathrm{q}} \Phi^{-1}\left(\mathrm{u}_{\mathrm{t}-\mathrm{j}}\right) \cdot \Phi^{-1}\left(\mathrm{v}_{\mathrm{t}-\mathrm{j}}\right)\right)
$$

where $\Lambda(\mathrm{x})=\left(1-\mathrm{e}^{-\mathrm{x}}\right)\left(1+\mathrm{e}^{-\mathrm{x}}\right)^{-1}$ is the modified logistic transformation that keeps the value of $\rho_{\mathrm{t}}$ in $(-1,1)$. The dependence parameter is explained by a constant, $\psi_{0}$, by an autoregressive term, $\psi_{1}$, and by the average product over the last q observations of the transformed variables, $\psi_{2}$. For the Student-t copula, $\Phi^{-1}(\mathrm{x})$ is substituted by 
$\mathrm{t}_{v}^{-1}(\mathrm{x})$. Similarly, we consider time-varying dependence for the Gumbel and its rotated version by assuming that their parameters follow the dynamics represented by the following equation:

$$
\delta_{\mathrm{t}}=\bar{\omega}+\bar{\beta} \delta_{\mathrm{t}-1}+\bar{\alpha} \frac{1}{\mathrm{q}} \sum_{\mathrm{j}=1}^{\mathrm{q}}\left|\mathrm{u}_{\mathrm{t}-\mathrm{j}}-\mathrm{v}_{\mathrm{t}-\mathrm{j}}\right| .
$$

Overall, the copula family considered here can be classified as either symmetric copulas with tail dependence (Student-t and time-varying Student-t copulas) or tail independence (Gaussian, time-varying Gaussian and Plackett) or as asymmetric copulas with tail dependence (Gumbel, rotated Gumbel, BB7, BB1, time-varying Gumbel and rotated Gumbel).

The parameters of the marginal and copula models are estimated using a twostep procedure called inference for the margins (Joe and Xu, 1996). The likelihood function is given by:

$$
f_{t}\left(R_{t}^{d}, R_{t}^{j}\right)=c(u, v) f_{R_{t}^{d}}\left(R_{t}^{d}\right) f_{R_{t}^{j}}\left(R_{t}^{j}\right),
$$

where $c(u, v)$ is the copula density and $f_{R_{t}^{d}}\left(R_{t}^{d}\right)$ and $f_{R_{t}^{j}}\left(R_{t}^{j}\right)$ are the marginal densities of $\mathrm{R}_{\mathrm{t}}^{\mathrm{d}}$ and $\mathrm{R}_{\mathrm{t}}^{\mathrm{j}}$, respectively. The $\log$ likelihood function can thus be decomposed as the sum of the log likelihood function of the marginals plus the log likelihood of the copula density. Thus, in a first step, we estimate the parameters of the marginal distributions separately by maximum likelihood and, in a second step, we estimate the parameters of the copula by solving the following problem:

$$
\theta=\arg \max _{\theta} \sum_{\mathrm{t}=1}^{\mathrm{T}} \ln \mathrm{c}\left(\hat{\mathrm{u}}_{\mathrm{t}}, \hat{\mathrm{v}}_{\mathrm{t}} ; \theta\right),
$$

where $\theta$ are the copula parameters and $\hat{u}_{t}=F_{R_{t}^{d}}\left(R_{t}^{d} ; \hat{\alpha}_{d}\right)$ and $\hat{v}_{t}=F_{R_{t}^{j}}\left(R_{t}^{j} ; \hat{\alpha}_{j}\right)$ are pseudo-sample observations for the copula. Under standard regularity conditions, this two-step estimation is consistent and the parameter estimates are asymptotically efficient and normal (see Joe, 1997). The number of lags in the mean and variance equations for each series is selected according to the Akaike information criteria (AIC) and the performance of the different copula models is evaluated using the AIC adjusted for small-sample bias, as in Breymann et al. (2003) and Reboredo (2011; 2013). 


\subsection{Data}

We empirically evaluated systemic risk in European sovereign debt markets by considering weekly data for sovereign bond benchmark price indices for France, Germany, the Netherlands, GIIPS markets and the overall sovereign bond price index for the EMU. Benchmark bond price indices were sourced from Datastream for 10-year maturities covering the period 7 January 2000 to 26 October 2012. Thus, we evaluated the VaR of the European sovereign debt market, represented by the EMU, conditional on the fact that a specific European debt market was in financial distress.

Figure 3.1 displays the benchmark bond price return dynamics (computed on a continuous compounding basis) for all the debt markets considered, and also for the EMU index returns, showing differences in the size and timing of price movements (especially relevant after the onset of the debt crisis at the end of 2009). Price volatility significantly changed for the GIIPS markets with the onset of the debt crisis, whereas volatility dynamics for the non-crisis countries (Germany, France and the Netherlands) remained relatively stable. Table 3.1 reports descriptive statistics for bond price returns. The average returns were similar across different debt markets and the corresponding standard deviations were larger for GIIPS markets than for the non-crisis markets. Also, differences between the maximum and minimum price returns show that price ranges were greater for GIIPS. Negative values for skewness were more pronounced for Greece than for the other debt markets (suggesting a greater probability of large decreases), with Ireland, Italy and Spain showing positive skewness. All return series showed high values for the kurtosis statistic, consistent with fat tails in the returns distributions; in fact, the Jarque-Bera test strongly rejected the normality of the unconditional distribution for all the series. The Ljung-Box statistic suggested the presence of serial correlation only in GIIPS returns. The autoregressive conditional heteroskedasticity-Lagrange multiplier (ARCH-LM) statistic indicated that ARCH effects could be found in all the returns series. Finally, the results of the Dickey and Fuller (1979) and Phillips and Perron (1988) non-stationarity tests and the Kwiatkowski et al. (1992) stationarity test confirm that all debt return series were stationary.

For the explanatory variables, we obtained weekly data on stock market indices for each country from Datastream, and also on the Eurostoxx 50 index for the EMU. Stock market volatility for each index at any time was computed as the standard deviation of the daily returns in a backward window of three months. Data 
for the Euribor at one year were also sourced from Datastream. Figure 3.2 depicts the stock price return dynamics, showing an abrupt change around the onset of the debt crisis that was common to all stock markets. This fact is corroborated by the behaviour of the market volatility data depicted in Figure 3.3. 
Figure 3.1: Time series plot of weekly bond returns for selected countries and the European Economic and Monetary Union (EMU).

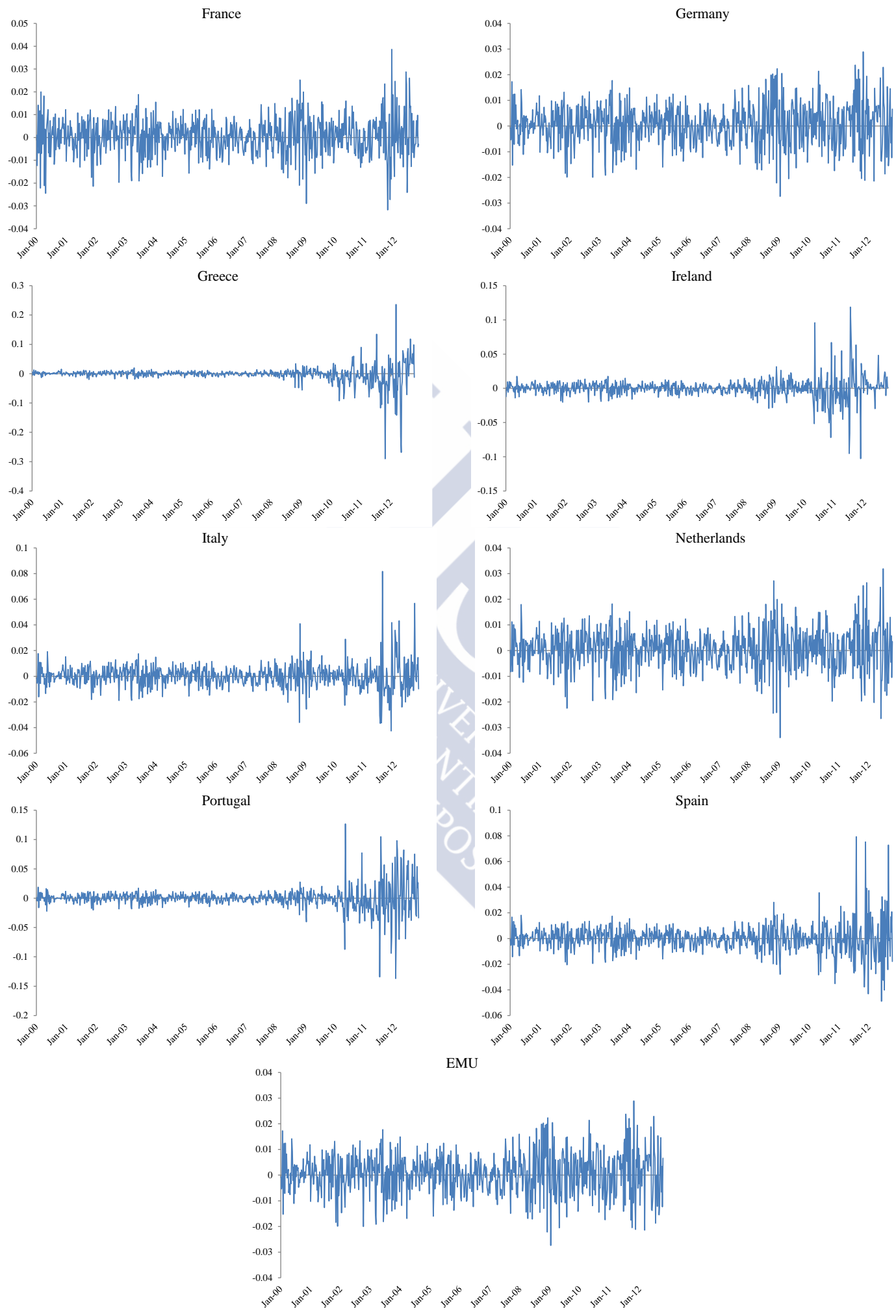


Table 3.1: Descriptive statistics for sovereign bond price returns.

\begin{tabular}{lccccccccc}
\hline & France & Germany & Greece & Ireland & Italy & Netherlands & Portugal & Spain & EMU \\
\hline Mean & 0.000 & 0.001 & -0.002 & 0.000 & 0.000 & 0.001 & 0.000 & 0.000 & 0.001 \\
Std. Dev. & 0.008 & 0.008 & 0.030 & 0.015 & 0.010 & 0.008 & 0.020 & 0.011 & 0.008 \\
Maximum & 0.039 & 0.029 & 0.236 & 0.119 & 0.082 & 0.032 & 0.127 & 0.079 & 0.029 \\
Minimum & -0.032 & -0.027 & -0.290 & -0.103 & -0.043 & -0.034 & -0.137 & -0.049 & -0.027 \\
Skewness & -0.063 & -0.073 & -2.401 & 0.393 & 1.168 & -0.166 & -0.034 & 1.061 & -0.072 \\
Kurtosis & 4.400 & 3.315 & 36.985 & 20.451 & 14.600 & 4.101 & 17.367 & 13.574 & 3.317 \\
J-B & $54.9^{*}$ & $3.3^{*}$ & $32788.7^{*}$ & $8493.9^{*}$ & $3897.4^{*}$ & $36.8^{*}$ & $5744.9^{*}$ & $3237.6^{*}$ & $3.4^{*}$ \\
Q(20) & 21.673 & 23.546 & 113.950 & 35.907 & 46.847 & 24.583 & 101.330 & 42.140 & 23.804 \\
& {$[0.359]$} & {$[0.263]$} & {$[0.000]$} & {$[0.016]$} & {$[0.001]$} & {$[0.218]$} & {$[0.000]$} & {$[0.003]$} & {$[0.251]$} \\
ARCH & 7.288 & 7.106 & 11.084 & 12.955 & 3.901 & 6.176 & 10.025 & 8.404 & 7.086 \\
& {$[0.000]$} & {$[0.000]$} & {$[0.000]$} & {$[0.000]$} & {$[0.000]$} & {$[0.000]$} & {$[0.000]$} & {$[0.000]$} & {$[0.000]$} \\
ADF & $-28.21^{*}$ & $-28.14^{*}$ & $-15.24^{*}$ & $-24.37^{*}$ & $-26.86^{*}$ & $-27.52^{*}$ & $-19.46^{*}$ & $-30.86^{*}$ & $-28.16^{*}$ \\
PP & $-28.19^{*}$ & $-28.13^{*}$ & $-23.32^{*}$ & $-24.34^{*}$ & $-26.84^{*}$ & $-27.52^{*}$ & $-25.86^{*}$ & $-30.68^{*}$ & -28.15 \\
KPSS & 0.06 & 0.10 & 0.50 & 0.08 & 0.05 & 0.08 & 0.16 & 0.17 & 0.10 \\
\hline
\end{tabular}

Notes. Weekly data for the period 14 January 2000 to 26 October 2012. EMU indicates European Economic and Monetary Union debt returns. J-B denotes the Jarque-Bera statistic for normality. Q $(\mathrm{k})$ is the LjungBox statistics for the serial correlation in returns computed with k lags. ARCH denotes Engle's LM test for heteroskedasticity computed using 20 lags. For these tests, p values are reported in square brackets. ADF, PP and KPSS are the empirical statistics of the Augmented Dickey-Fuller (1979) unit root test, the PhillipsPerron (1988) unit root test, and the Kwiatkowski et al. (1992) stationarity test, respectively. An asterisk

(*) indicates rejection of the null hypothesis at $5 \%$. 
Figure 3.2: Time series plot of weekly market index returns for indices for selected countries and the European Economic and Monetary Union (EMU).
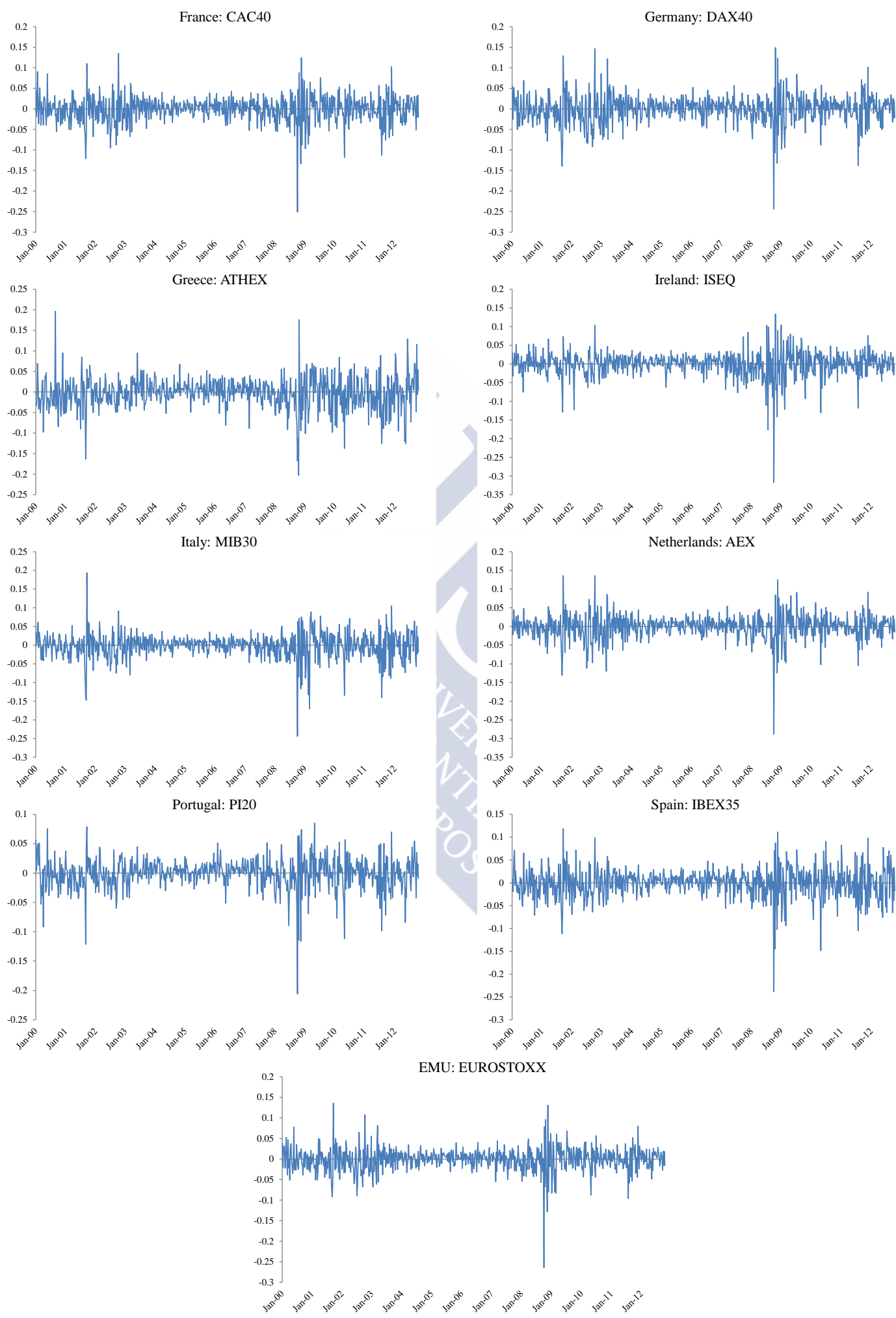
Figure 3.3: Time series plot of weekly market volatility returns for selected countries and the Eurostoxx 50.
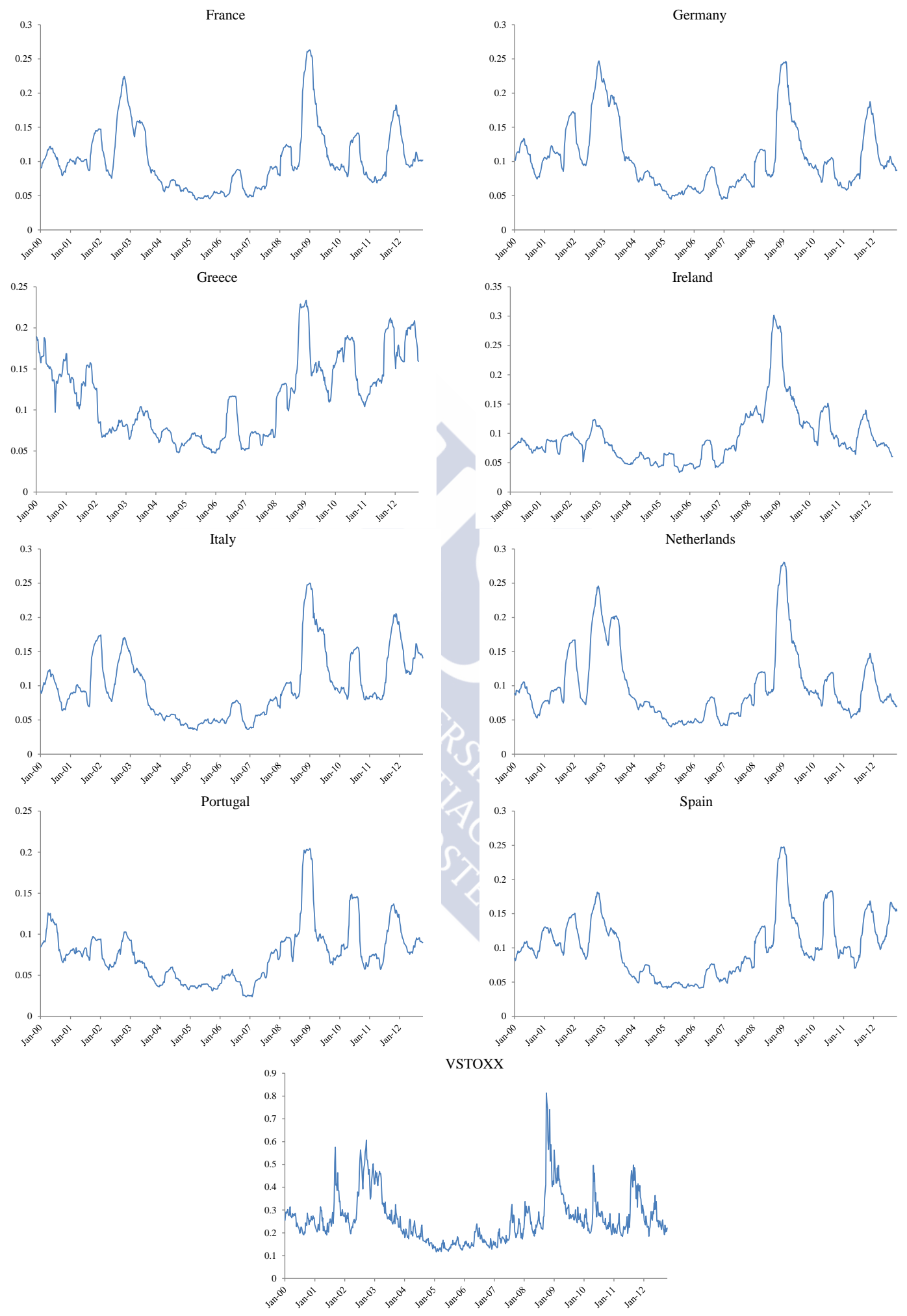
Table 3.3 reports descriptive statistics for stock market return volatility and interest rate changes. Average volatility is similar across stock markets, with all volatility series exhibiting positive skewness and kurtosis, uncorrelated volatility and $\mathrm{ARCH}$ effects. The main features of log changes in the interest rate series are negative skewness, kurtosis and ARCH effects.

Finally, we considered the crisis dummy variable, identifying two sample periods: before and after the onset of the European sovereign debt crisis (hereafter, the pre- and post-onset periods, respectively), assigning the values 0 to the pre-onset period and 1 to the post-onset period. The crucial point here was to determine the date when the European sovereign debt crisis started. Following Bhanot et al. (2014), we took this date to be November 2009. This was when investors became concerned regarding the quality of Greek debt, in response to the Greek government revealing that its debt amounted to $12.7 \%$ of gross domestic product, a figure considerably superior to the previously announced figure of $6.7 \%$. The fact that the impact of the crisis was different across debt markets is likely to affect the dependence relationship between markets. Table 3.4 illustrates how the values of the Pearson correlation coefficient changed with the advent of the crisis. The lower triangular matrix shows that the correlation coefficient was positive and high for all the market pairs; however, the upper triangular matrix shows how correlation coefficients changed dramatically with the onset of the debt crisis: GIIPS negatively correlated with the EMU index, whereas France, Germany and the Netherlands continued to show high dependence on this index. Furthermore, linear dependence of the Greek market with the other markets changed, which decoupled from the noncrisis markets and reduced dependence on the other countries in crisis. 
Table 3.2: Descriptive statistics for stock market returns.

\begin{tabular}{lccccccccc}
\hline & France & Germany & Greece & Ireland & Italy & Netherlands & Portugal & Spain & EMU \\
& CAC40 & DAX40 & ATHEX & ISEQ & MIB30 & AEX & PI20 & IBEX35 & Eurostoxx 50 \\
\hline Mean & -0.001 & 0.000 & -0.003 & -0.001 & -0.001 & -0.001 & -0.001 & -0.001 & -0.001 \\
Std. Dev. & 0.033 & 0.035 & 0.040 & 0.034 & 0.035 & 0.034 & 0.027 & 0.034 & 0.030 \\
Maximum & 0.135 & 0.149 & 0.196 & 0.134 & 0.194 & 0.136 & 0.085 & 0.118 & 0.136 \\
Minimum & -0.251 & -0.243 & -0.203 & -0.317 & -0.244 & -0.288 & -0.206 & -0.238 & -0.264 \\
Skewness & -0.814 & -0.553 & -0.288 & -1.707 & -0.915 & -1.081 & -1.212 & -0.766 & -1.016 \\
Kurtosis & 9.322 & 8.067 & 6.192 & 16.100 & 9.648 & 11.866 & 10.072 & 7.872 & 13.876 \\
J-B & 1186.26 & 748.69 & 292.93 & 5100.83 & 1323.28 & 2317.97 & 1555.68 & 726.07 & 3407.52 \\
Q(20) & 45.444 & 34.658 & 30.672 & 45.025 & 39.934 & 36.632 & 30.402 & 37.335 & 64.862 \\
& {$[0.001]$} & {$[0.022]$} & {$[0.060]$} & {$[0.001]$} & {$[0.005]$} & {$[0.013]$} & {$[0.064]$} & {$[0.011]$} & {$[0.000]$} \\
ARCH & 3.550 & 5.868 & 4.848 & 5.541 & 5.462 & 2.616 & 4.563 & 4.344 & 3.718 \\
& {$[0.000]$} & {$[0.000]$} & {$[0.000]$} & {$[0.000]$} & {$[0.000]$} & {$[0.000]$} & {$[0.000]$} & {$[0.000]$} & {$[0.000]$}
\end{tabular}

Notes. See Table 3.1 notes. 
Table 3.3: Descriptive statistics for market volatility and Euribor at 1 year.

\begin{tabular}{lcccccccccc}
\hline & France & Germany & Greece & Ireland & Italy & Netherlands & Portugal & Spain & EMU & Euribor 1y \\
\hline Mean & 0.103 & 0.106 & 0.116 & 0.094 & 0.100 & 0.100 & 0.076 & 0.102 & 0.261 & 0.000 \\
Std. Dev. & 0.045 & 0.048 & 0.048 & 0.047 & 0.048 & 0.052 & 0.035 & 0.044 & 0.106 & 0.001 \\
Maximum & 0.263 & 0.247 & 0.234 & 0.302 & 0.250 & 0.281 & 0.205 & 0.248 & 0.813 & 0.003 \\
Minimum & 0.044 & 0.045 & 0.047 & 0.034 & 0.035 & 0.040 & 0.024 & 0.041 & 0.116 & -0.003 \\
Skewness & 1.280 & 1.178 & 0.426 & 2.066 & 0.915 & 1.497 & 1.272 & 0.855 & 1.540 & -0.468 \\
Kurtosis & 4.712 & 3.740 & 2.130 & 8.599 & 3.374 & 4.855 & 5.467 & 3.790 & 6.238 & 6.835 \\
J-B & $264.06^{*}$ & $169.69^{*}$ & $41.27^{*}$ & $1347.99^{*}$ & $97.15^{*}$ & $345.17^{*}$ & $349.50^{*}$ & $98.70^{*}$ & $556.05^{*}$ & $433.73^{*}$ \\
Q(20) & 7600.1 & 8054.8 & 8983.6 & 9428.9 & 8098.5 & 7964.8 & 7634.3 & 7518.3 & 5613.7 & 442.53 \\
& {$[0.00]$} & {$[0.00]$} & {$[0.00]$} & {$[0.00]$} & {$[0.00]$} & {$[0.00]$} & {$[0.00]$} & {$[0.00]$} & {$[0.00]$} & {$[0.00]$} \\
ARCH & 2382.7 & 1974.5 & 1047.3 & 3307.9 & 1831.2 & 3532.8 & 2140.5 & 2212.1 & 69.9 & 9.6 \\
& {$[0.00]$} & {$[0.00]$} & {$[0.00]$} & {$[0.00]$} & {$[0.00]$} & {$[0.00]$} & {$[0.00]$} & {$[0.00]$} & {$[0.00]$} & {$[0.00]$} \\
\hline
\end{tabular}

Notes. See Table 3.1 notes. 
Table 3.4: Pearson correlation coefficients.

\begin{tabular}{lccccccccc}
\hline & France & Germany & Greece & Ireland & Italy & Netherlands & Portugal & Spain & EMU \\
\hline France & & 0.69 & -0.09 & 0.09 & 0.22 & 0.80 & -0.02 & 0.23 & 0.69 \\
Germany & 0.95 & & -0.36 & -0.10 & -0.19 & 0.93 & -0.16 & -0.09 & 1.00 \\
Greece & 0.81 & 0.78 & & 0.27 & 0.26 & -0.28 & 0.29 & 0.22 & -0.36 \\
Ireland & 0.82 & 0.80 & 0.85 & & 0.40 & 0.00 & 0.51 & 0.35 & -0.10 \\
Italy & 0.89 & 0.87 & 0.91 & 0.86 & & -0.08 & 0.29 & 0.75 & -0.19 \\
Netherlands & 0.95 & 0.96 & 0.86 & 0.86 & 0.92 & & -0.12 & 0.00 & 0.93 \\
Portugal & 0.90 & 0.89 & 0.91 & 0.88 & 0.94 & 0.94 & & 0.25 & -0.16 \\
Spain & 0.94 & 0.94 & 0.86 & 0.87 & 0.93 & 0.97 & 0.95 & & -0.09 \\
EMU & 0.95 & 1.00 & 0.78 & 0.80 & 0.87 & 0.96 & 0.89 & 0.94 & \\
\hline
\end{tabular}

Notes. EMU indicates European Economic and Monetary Union. The lower triangular matrix reports the Pearson's correlation coefficient in the pre-onset period and the upper triangular matrix shows the Pearson's correlation coefficient in the post-onset period.

\subsection{Empirical results}

\subsubsection{Marginal model results}

Table 3.5 displays estimation results for the marginal models specified in Eqs. (3.8)(3.10) for sovereign debt returns. Marginal models were estimated by considering different combinations of the parameters $\mathrm{p}, \mathrm{r}$ and $\mathrm{m}$ for values ranging from zero to a maximum lag of two; the most suitable model was selected according to AIC values. The evidence reported in Table 3.5 indicates that stock market returns had a negative impact on sovereign debt returns for all debt markets except Greece, Ireland, Italy and Portugal; stock market volatility, meanwhile, had no significant impact on average returns except for French debt returns and for the EMU index. Interest rate dynamics had a significant negative impact on debt returns in all series. The results for the crisis dummy variable indicate that the sovereign debt crisis negatively impacted average returns only in Greece and Portugal. Regarding debt return volatility, the empirical results confirm that volatility was persistent across different debt markets and leverage effects were hardly observed (Ireland, Italy and Spain were the exceptions). Consistent with the descriptive evidence on nonnormality and fat tails reported in Table 3.1, the estimated values for the degrees of freedom and the symmetry parameter of the skewed Student-t distribution confirm that the error terms are not normal and in some cases are asymmetric.

We also checked the goodness-of-fit of our marginal models. The last rows of Table 3.5 indicate that neither autocorrelation nor $\mathrm{ARCH}$ effects remain in the residuals of the marginal models. Furthermore, we tested for the adequacy of the 
skewed-t distribution model, testing the null hypothesis that the standardized model residuals were uniform $(0,1)$ by comparing the empirical distribution and the theoretical distribution function using the well-known Kolmogorov-Smirnov, Cramér-von Mises and Anderson-Darling tests. The p-values for these tests, reported in last three rows of Table 3.5, indicate that, for either of the marginal models, the null of the correct specification of the distribution function could not be rejected at the $5 \%$ significance level. Overall, our goodness-of-fit tests indicate that the marginal distribution models were not mis-specified, so the copula model could correctly capture dependence between debt markets.

\subsubsection{Copula model results}

We considered the potential effects of the European sovereign debt crisis on dependence by estimating copula models for the pre- and post-onset periods, delimitating both periods according to the information reported by the crisis dummy variable. We also considered the systemic risk of each country for the European debt market as a whole and the systemic risk of Greece - the main country affected by the debt crisis - for other European debt markets. For the pre-onset and post-onset periods we estimated eight copula pairs for the EMU index returns and each country's debt index returns; we also estimated seven copula pairs for the Greek debt index returns and the debt market returns for each of the other countries.

Tables 3.6 and 3.7 report the copula model results for the EMU paired with each European country in the pre- and post-onset periods, respectively. The evidence from both static and time-varying copulas indicate that all the debt markets strongly co-moved with the EMU index, providing consistent evidence of tail dependence and time-varying (TVP) dependence. In fact, according to the AIC values, TVP-rotated Gumbel copula and the Student-t offered the best fit for all markets, meaning that there was lower tail dependence and, in one case, symmetric tail dependence. Table 3.7 reports the results for the post-onset period, showing how the shape of dependence changed completely. With the onset of the crisis, European debt markets decoupled: GIIPS debt markets moved in the opposite direction to the general debt index, whereas the non-crisis countries (France, Germany and the Netherlands) continued to strongly co-move (mainly Germany) with the EMU index as in the pre-onset period. Tail dependence results also indicated that Greece and Portugal decoupled from the EMU index under extreme market movements, since, according to the AIC, the best copula fit displayed tail independence (the Gaussian copula for Greece and the Plackett copula for Portugal). The other countries in 
crisis displayed lower symmetric tail dependence - as represented by the TVPStudent-t copula - in the post-onset period compared to the pre-onset period. For the non-crisis countries, tail dependence also changed, with France and the Netherlands showing lower tail independence and with Germany showing asymmetric tail dependence. Obviously, such changes in the dependence structure, and in particular in tail dependence, have implications for systemic risk that will be considered below.

Tables 3.8 and 3.9 report results for the copula models for Greece paired with each European country in the pre- and post-onset periods. The evidence provided by the static and TVP copulas shows that the Greek debt market strongly co-moved with all the European debt markets, indicating lower tail dependence and upper tail independence. According to the AIC, the TVP-rotated Gumbel copula was the model that best fit all countries, with the exception of Italy, where the TVP-Gumbel copula offered the best fit, displaying upper tail dependence and lower tail independence with Greece. Table 3.9 reports dependence results for the post-onset period, showing that Greek debt market dependence on other European markets completely changed with the onset of the debt crisis. The Greek market decoupled from the non-crisis countries (France, Germany and the Netherlands), moving in the opposite direction on average and showing independence at the tails. The Greek debt market continued to co-move with the other countries in crisis in the post-onset period, although the intensity of co-movement decreased considerably. According to the AIC, the best copula fit revealed that, in times of extreme downturns, the Greek debt market did not co-move with the Irish, Italian, Portuguese or Spanish debt markets. There was also upper tail independence with the Greek market for all these markets except Portugal. It is important to point out that, according to our copula results, the Greek crisis at its depth did not have any spillover effect on the general EMU market index, given that this index is mainly based on non-crisis countries. This evidence is consistent with the results reported in Bhanot et al. (2014) for a multivariate GARCH model. 
Table 3.5: Parameter estimates for the marginal distribution models.

\begin{tabular}{|c|c|c|c|c|c|c|c|c|c|}
\hline & France & Germany & Greece & Ireland & Italy & Netherlands & Portugal & Spain & EMU \\
\hline \multicolumn{10}{|l|}{ Mean } \\
\hline$\phi_{0}$ & $\begin{array}{c}0.001^{*} \\
(2.18)\end{array}$ & $\begin{array}{c}0.001^{*} \\
(1.99)\end{array}$ & $\begin{array}{l}0.001 \\
(0.85)\end{array}$ & $\begin{array}{l}0.000 \\
(0.37)\end{array}$ & $\begin{array}{l}0.001 \\
(1.77)\end{array}$ & $\begin{array}{l}0.001 \\
(1.58)\end{array}$ & $\begin{array}{c}0.001^{*} \\
(2.10)\end{array}$ & $\begin{array}{c}0.001^{*} \\
(1.96)\end{array}$ & $\begin{array}{c}0.001^{*} \\
(2.04)\end{array}$ \\
\hline $\operatorname{AR}(1)$ & $\begin{array}{c}-0.112^{*} \\
(-2.64)\end{array}$ & $\begin{array}{c}-0.091^{*} \\
(-2.18)\end{array}$ & & $\begin{array}{l}-0.042 \\
(-1.00)\end{array}$ & & & $\begin{array}{r}-0.125^{*} \\
(-3.07)\end{array}$ & $\begin{array}{c}-0.121^{*} \\
(-2.99)\end{array}$ & $\begin{array}{c}-0.094^{*} \\
(-2.27)\end{array}$ \\
\hline Euribor & $\begin{array}{c}-4.726^{*} \\
(-8.97)\end{array}$ & $\begin{array}{l}-4.388^{*} \\
(-9.03)\end{array}$ & $\begin{array}{l}-4.754^{*} \\
(-11.24)\end{array}$ & $\begin{array}{c}-4.957^{*} \\
(-9.78)\end{array}$ & $\begin{array}{l}-4.673^{*} \\
(-11.60)\end{array}$ & $\begin{array}{c}-4.429 * \\
(-9.70)\end{array}$ & $\begin{array}{l}-5.017^{*} \\
(-10.47)\end{array}$ & $\begin{array}{l}-4.824^{*} \\
(-11.28)\end{array}$ & $\begin{array}{r}-4.574^{*} \\
(-9.07)\end{array}$ \\
\hline EMU Index & $\begin{array}{c}-0.068^{*} \\
(-5.74)\end{array}$ & $\begin{array}{c}-0.069^{*} \\
(-6.30)\end{array}$ & $\begin{array}{l}-0.003 \\
(-0.39)\end{array}$ & $\begin{array}{l}-0.013 \\
(-1.06)\end{array}$ & $\begin{array}{l}-0.012 \\
(-0.91)\end{array}$ & $\begin{array}{c}-0.068^{*} \\
(-5.91)\end{array}$ & $\begin{array}{l}-0.014 \\
(-1.05)\end{array}$ & $\begin{array}{c}-0.026^{*} \\
(-2.33)\end{array}$ & $\begin{array}{c}-0.091^{*} \\
(-6.21)\end{array}$ \\
\hline Market Vol. & $\begin{array}{c}-0.011^{*} \\
(-1.92)\end{array}$ & $\begin{array}{l}-0.009 \\
(-1.93)\end{array}$ & $\begin{array}{l}-0.003 \\
(-0.45)\end{array}$ & $\begin{array}{l}0.000 \\
(0.05)\end{array}$ & $\begin{array}{l}-0.008 \\
(-1.68)\end{array}$ & $\begin{array}{l}-0.007 \\
(-1.29)\end{array}$ & $\begin{array}{l}-0.015 \\
(-1.82)\end{array}$ & $\begin{array}{l}-0.008 \\
(-1.49)\end{array}$ & $\begin{array}{c}-0.005^{*} \\
(-2.03)\end{array}$ \\
\hline Dummy & $\begin{array}{c}0.001 \\
(1.197)\end{array}$ & $\begin{array}{l}0.001 \\
(1.50)\end{array}$ & $\begin{array}{c}-0.012^{*} \\
(-4.32)\end{array}$ & $\begin{array}{l}-0.001 \\
(-0.90)\end{array}$ & $\begin{array}{c}0.000 \\
(-0.23)\end{array}$ & $\begin{array}{l}0.001 \\
(1.49)\end{array}$ & $\begin{array}{c}-0.003^{*} \\
(-2.16)\end{array}$ & $\begin{array}{l}-0.001 \\
(-1.33)\end{array}$ & $\begin{array}{l}0.001 \\
(1.72)\end{array}$ \\
\hline \multicolumn{10}{|l|}{ Variance } \\
\hline$\omega$ & $\begin{array}{l}1.968^{*} \\
(2.35)\end{array}$ & $\begin{array}{l}1.531^{*} \\
(2.35)\end{array}$ & $\begin{array}{c}0.035^{*} \\
(3.74)\end{array}$ & $\begin{array}{l}0.019 * \\
(2.16)\end{array}$ & $\begin{array}{l}1.291^{*} \\
(2.54)\end{array}$ & $\begin{array}{l}1.870 * \\
(2.073)\end{array}$ & $\begin{array}{c}0.014^{*} \\
(3.05)\end{array}$ & $\begin{array}{c}0.015^{*} \\
(2.64)\end{array}$ & $\begin{array}{l}1.567^{*} \\
(2.27)\end{array}$ \\
\hline$\alpha_{1}$ & $\begin{array}{c}0.087^{*} \\
(3.04)\end{array}$ & $\begin{array}{c}0.085^{*} \\
(2.68)\end{array}$ & $\begin{array}{l}0.034 \\
(0.50)\end{array}$ & $\begin{array}{l}-0.003 \\
(-0.08)\end{array}$ & $\begin{array}{l}0.000 \\
(0.01)\end{array}$ & $\begin{array}{l}0.087^{*} \\
(2.543)\end{array}$ & $\begin{array}{l}0.038 \\
(1.47)\end{array}$ & $\begin{array}{l}0.010 \\
(0.38)\end{array}$ & $\begin{array}{c}0.094^{*} \\
(2.72)\end{array}$ \\
\hline$\beta_{1}$ & $\begin{array}{l}0.867^{*} \\
(29.61)\end{array}$ & $\begin{array}{l}0.885^{*} \\
(37.38)\end{array}$ & $\begin{array}{l}0.851^{*} \\
(21.51)\end{array}$ & $\begin{array}{l}0.884^{*} \\
(20.26)\end{array}$ & $\begin{array}{l}0.890^{*} \\
(40.50)\end{array}$ & $\begin{array}{l}0.863^{*} \\
(24.11)\end{array}$ & $\begin{array}{l}0.886^{*} \\
(48.30)\end{array}$ & $\begin{array}{l}0.882^{*} \\
(33.18)\end{array}$ & $\begin{array}{l}0.879^{*} \\
(34.66)\end{array}$ \\
\hline$\lambda$ & $\begin{array}{l}0.002 \\
(0.04)\end{array}$ & $\begin{array}{l}-0.020 \\
(-0.49)\end{array}$ & $\begin{array}{l}0.158 \\
(1.29)\end{array}$ & $\begin{array}{c}0.162^{*} \\
(3.45)\end{array}$ & $\begin{array}{c}0.166^{*} \\
(3.37)\end{array}$ & $\begin{array}{c}0.005 \\
(0.094)\end{array}$ & $\begin{array}{c}0.104 \\
(1.840)\end{array}$ & $\begin{array}{l}0.147^{*} \\
(2.226)\end{array}$ & $\begin{array}{l}-0.028 \\
(-0.65)\end{array}$ \\
\hline Dummy & $\begin{array}{l}0.000^{*} \\
(32.81)\end{array}$ & $\begin{array}{l}0.000^{*} \\
(99.43)\end{array}$ & $\begin{array}{l}0.000^{*} \\
(87.63)\end{array}$ & $\begin{array}{l}0.000^{*} \\
(151.6)\end{array}$ & $\begin{array}{l}0.000^{*} \\
(42.44)\end{array}$ & $\begin{array}{l}0.000^{*} \\
(75.75)\end{array}$ & $\begin{array}{c}0.000^{*} \\
(227.2)\end{array}$ & $\begin{array}{l}0.000^{*} \\
(186.3)\end{array}$ & $\begin{array}{l}0.000^{*} \\
(93.75)\end{array}$ \\
\hline Asymmetry & $\begin{array}{l}-0.034 \\
(-0.61)\end{array}$ & $\begin{array}{c}-0.116^{*} \\
(-2.31)\end{array}$ & $\begin{array}{c}-0.165^{*} \\
(-2.68)\end{array}$ & $\begin{array}{c}-0.115^{*} \\
(-2.12)\end{array}$ & $\begin{array}{l}-0.117 \\
(-1.85)\end{array}$ & $\begin{array}{l}-0.073 \\
(-1.26)\end{array}$ & $\begin{array}{c}-0.127^{*} \\
(-1.93)\end{array}$ & $\begin{array}{c}-0.144^{*} \\
(-2.48)\end{array}$ & $\begin{array}{l}-0.091 \\
(-1.69)\end{array}$ \\
\hline Tail & $\begin{array}{l}6.827^{*} \\
(72.08)\end{array}$ & $\begin{array}{l}8.538^{*} \\
(39.20)\end{array}$ & $\begin{array}{c}3.348^{*} \\
(4.02)\end{array}$ & $\begin{array}{l}5.257^{*} \\
(14.36)\end{array}$ & $\begin{array}{l}6.424^{*} \\
(105.6)\end{array}$ & $\begin{array}{c}8.390^{*} \\
(117.2)\end{array}$ & $\begin{array}{l}5.614^{*} \\
(39.93)\end{array}$ & $\begin{array}{l}7.252^{*} \\
(83.69)\end{array}$ & $\begin{array}{l}8.489^{*} \\
(128.8)\end{array}$ \\
\hline LogLik & 2404.47 & 2412.65 & 2120.94 & 2259.65 & 2390.82 & 2428.22 & 2203.12 & 2332.55 & 2414.79 \\
\hline$L J$ & $\begin{array}{l}18.129 \\
{[0.514]}\end{array}$ & $\begin{array}{l}16.061 \\
{[0.653]}\end{array}$ & $\begin{array}{l}24.227 \\
{[0.233]}\end{array}$ & $\begin{array}{l}16.418 \\
{[0.629]}\end{array}$ & $\begin{array}{l}29.691 \\
{[0.075]}\end{array}$ & $\begin{array}{l}19.782 \\
{[0.472]}\end{array}$ & $\begin{array}{l}18.374 \\
{[0.498]}\end{array}$ & $\begin{array}{l}19.710 \\
{[0.412]}\end{array}$ & $\begin{array}{l}17.921 \\
{[0.528]}\end{array}$ \\
\hline$L J^{2}$ & $\begin{array}{l}22.954 \\
{[0.192]}\end{array}$ & $\begin{array}{l}23.810 \\
{[0.161]}\end{array}$ & $\begin{array}{l}27.523 \\
{[0.051]}\end{array}$ & $\begin{array}{l}27.278 \\
{[0.074]}\end{array}$ & $\begin{array}{l}15.443 \\
{[0.631]}\end{array}$ & $\begin{array}{l}19.416 \\
{[0.367]}\end{array}$ & $\begin{array}{l}21.691 \\
{[0.246]}\end{array}$ & $\begin{array}{l}12.057 \\
{[0.844]}\end{array}$ & $\begin{array}{l}26.933 \\
{[0.080]}\end{array}$ \\
\hline $\mathrm{ARCH}$ & $\begin{array}{c}1.065 \\
{[0.383]}\end{array}$ & $\begin{array}{c}1.403 \\
{[0.113]}\end{array}$ & $\begin{array}{c}1.456 \\
{[0.090]}\end{array}$ & $\begin{array}{c}1.365 \\
{[0.133]}\end{array}$ & $\begin{array}{c}0.826 \\
{[0.682]}\end{array}$ & $\begin{array}{c}0.969 \\
{[0.498]}\end{array}$ & $\begin{array}{c}0.939 \\
{[0.537]}\end{array}$ & $\begin{array}{c}0.603 \\
{[0.912]}\end{array}$ & $\begin{array}{c}1.549 \\
{[0.060]}\end{array}$ \\
\hline K-S & {$[0.986]$} & {$[0.946]$} & {$[0.967]$} & {$[0.892]$} & {$[0.882]$} & {$[0.903]$} & {$[0.940]$} & {$[0.948]$} & {$[0.531]$} \\
\hline C-vM & {$[0.990]$} & {$[0.864]$} & {$[0.938]$} & {$[0.812]$} & [0.989] & {$[0.926]$} & {$[0.989]$} & {$[0.996]$} & {$[0.740]$} \\
\hline A-D & {$[0.992]$} & {$[0.911]$} & {$[0.963]$} & {$[0.896]$} & {$[0.998]$} & {$[0.907]$} & {$[0.992]$} & {$[0.999]$} & {$[0.806]$} \\
\hline
\end{tabular}

Note. The table presents the maximum likelihood estimates and the $\mathrm{z}$ statistics (in parentheses) for the parameters of the marginal distribution models given by Eqs. (8)-(10). LogLik is the log-likelihood value. LJ denotes the Ljung-Box statistic for serial correlation in the residual model calculated with 20 lags. LJ ${ }^{2}$ denotes the Ljung-Box statistic for serial correlation in the squared residual model calculated with 20 lags. ARCH is Engle's LM test for the ARCH effect in the residuals up to 20th order. K-S, C-vM and A-D denote the Kolmogorov-Smirnov, Cramér-von Mises and Anderson-Darling tests for adequacy of the skewed-t distribution model. $\mathrm{P}$ values (in square brackets) below 0.05 indicate rejection of the null hypothesis. An asterisk $(*)$ indicates significance at 5\%. EMU indicates European Economic and Monetary Union. 
Table 3.6: Estimates for copula models in the period before crisis onset. European Economic and Monetary Union (EMU) vs. selected countries.

Panel A: Parameter estimates for time-invariant copulas.

\begin{tabular}{|c|c|c|c|c|c|c|c|c|}
\hline Copula & France & Germany & Greece & Ireland & Italy & Netherlands & Portugal & Spain \\
\hline \multicolumn{9}{|c|}{ Gaussian } \\
\hline$\rho$ & $\begin{array}{r}0.936^{*} \\
(0.00)\end{array}$ & $\begin{array}{r}0.981^{*} \\
(0.00)\end{array}$ & $\begin{array}{r}0.834^{*} \\
(0.01)\end{array}$ & $\begin{array}{r}0.835^{*} \\
(0.10)\end{array}$ & $\begin{array}{r}0.868^{*} \\
(0.01)\end{array}$ & $\begin{array}{r}0.943^{*} \\
(0.00)\end{array}$ & $\begin{array}{r}0.880^{*} \\
(0.01)\end{array}$ & $\begin{array}{r}0.909^{*} \\
(0.01)\end{array}$ \\
\hline AIC & -1067.493 & -1680.860 & -603.613 & -608.745 & -713.692 & -1123.293 & -756.375 & -890.382 \\
\hline \multicolumn{9}{|c|}{ Student-t } \\
\hline$\rho$ & $\begin{array}{r}0.952^{*} \\
(0.01)\end{array}$ & $\begin{array}{r}0.983^{*} \\
(0.00)\end{array}$ & $\begin{array}{r}0.858^{*} \\
(0.01)\end{array}$ & $\begin{array}{r}0.868^{*} \\
(0.01)\end{array}$ & $\begin{array}{r}0.881^{*} \\
(0.01)\end{array}$ & $\begin{array}{r}0.948^{*} \\
(0.00)\end{array}$ & $\begin{array}{r}0.897^{*} \\
(0.01)\end{array}$ & $\begin{array}{r}0.916^{*} \\
(0.01)\end{array}$ \\
\hline$v$ & $\begin{array}{r}1.828^{*} \\
(0.26)\end{array}$ & $\begin{array}{r}3.510^{*} \\
(0.80)\end{array}$ & $\begin{array}{r}2.841^{*} \\
(0.53)\end{array}$ & $\begin{array}{r}2.322^{*} \\
(0.36)\end{array}$ & $\begin{array}{r}4.214^{*} \\
(0.99)\end{array}$ & $\begin{array}{r}2.495^{*} \\
(0.36)\end{array}$ & $\begin{array}{r}3.247^{*} \\
(0.59)\end{array}$ & $\begin{array}{r}3.646^{*} \\
(0.82)\end{array}$ \\
\hline AIC & -1266.306 & -1760.424 & -688.291 & -724.814 & -759.001 & -1214.324 & -824.799 & -936.630 \\
\hline \multicolumn{9}{|l|}{ Gumbel } \\
\hline$\delta$ & $\begin{array}{r}4.674^{*} \\
(0.18)\end{array}$ & $\begin{array}{r}7.955^{*} \\
(0.30)\end{array}$ & $\begin{array}{c}2.764^{*} \\
(0.10)\end{array}$ & $\begin{array}{r}2.840^{*} \\
(0.11)\end{array}$ & $\begin{array}{r}3.001^{*} \\
(0.11)\end{array}$ & $\begin{array}{c}4.593^{*} \\
(0.17)\end{array}$ & $\begin{array}{r}3.174^{*} \\
(0.12)\end{array}$ & $\begin{array}{r}3.528^{*} \\
(0.13)\end{array}$ \\
\hline $\mathrm{AIC}$ & -1117.217 & -1687.119 & -619.432 & -643.766 & -715.031 & -1115.740 & -748.857 & -865.385 \\
\hline \multicolumn{9}{|c|}{ Rotated Gumbel } \\
\hline$\delta$ & $\begin{array}{l}4.971^{*} \\
(0.19)\end{array}$ & $\begin{array}{c}7.969^{*} \\
(0.30)\end{array}$ & $\begin{array}{r}2.809^{*} \\
(0.10)\end{array}$ & $\begin{array}{c}2.862^{*} \\
(0.11)\end{array}$ & $\begin{array}{r}2.981^{*} \\
(0.11)\end{array}$ & $\begin{array}{l}4.759^{*} \\
(0.18)\end{array}$ & $\begin{array}{r}3.270^{*} \\
(0.12)\end{array}$ & $\begin{array}{r}3.662^{*} \\
(0.14)\end{array}$ \\
\hline AIC & -1186.789 & -1688.961 & -644.116 & -657.189 & -704.506 & -1166.714 & -784.269 & -901.423 \\
\hline \multicolumn{9}{|l|}{ BB7 } \\
\hline$\theta$ & $\begin{array}{r}4.162^{*} \\
(0.15)\end{array}$ & $\begin{array}{c}4.929^{*} \\
(0.00)\end{array}$ & $\begin{array}{c}2.421^{*} \\
(0.25)\end{array}$ & $\begin{array}{r}2.563^{*} \\
(0.18)\end{array}$ & $\begin{array}{r}2.801^{*} \\
(0.29)\end{array}$ & $\begin{array}{c}4.116^{*} \\
(0.22)\end{array}$ & $\begin{array}{l}2.711 \\
(2.14)\end{array}$ & $\begin{array}{r}3.073^{*} \\
(0.49)\end{array}$ \\
\hline$\delta$ & $\begin{array}{r}4.770^{*} \\
(0.27)\end{array}$ & $\begin{array}{c}8.747^{*} \\
(0.03)\end{array}$ & $\begin{array}{c}2.051^{*} \\
(0.39)\end{array}$ & $\begin{array}{r}1.930^{*} \\
(0.18)\end{array}$ & $\begin{array}{r}1.877^{*} \\
(0.22)\end{array}$ & $\begin{array}{r}4.461^{*} \\
(0.31)\end{array}$ & $\begin{array}{r}2.469^{*} \\
(1.22)\end{array}$ & $\begin{array}{r}2.937^{*} \\
(0.85)\end{array}$ \\
\hline AIC & -1136.301 & -1604.134 & -633.823 & -645.491 & -696.002 & -1131.798 & -748.137 & -870.589 \\
\hline \multicolumn{9}{|c|}{ Plackett } \\
\hline$\theta$ & $\begin{array}{r}134.543^{*} \\
(13.34)\end{array}$ & $\begin{array}{r}294.951^{*} \\
(32.99)\end{array}$ & $\begin{array}{r}31.904^{*} \\
(3.49)\end{array}$ & $\begin{array}{r}40.619^{*} \\
(4.60)\end{array}$ & $\begin{array}{r}37.994^{*} \\
(3.25)\end{array}$ & $\begin{array}{r}110.522^{*} \\
(15.88)\end{array}$ & $\begin{array}{r}46.854^{*} \\
(4.46)\end{array}$ & $\begin{array}{r}56.622^{*} \\
(5.43)\end{array}$ \\
\hline AIC & -1256.681 & -1691.174 & -672.451 & -733.420 & -755.312 & -1189.036 & -827.383 & -921.424 \\
\hline \multicolumn{9}{|l|}{ BB1 } \\
\hline$\theta$ & $\begin{array}{r}1.051^{*} \\
(0.15)\end{array}$ & $\begin{array}{r}0.827^{*} \\
(0.13)\end{array}$ & $\begin{array}{r}0.679^{*} \\
(0.12)\end{array}$ & $\begin{array}{r}0.599^{*} \\
(0.12)\end{array}$ & $\begin{array}{r}0.479^{*} \\
(0.11)\end{array}$ & $\begin{array}{r}0.947^{*} \\
(0.14)\end{array}$ & $\begin{array}{r}0.730^{*} \\
(0.12)\end{array}$ & $\begin{array}{r}0.783^{*} \\
(0.13)\end{array}$ \\
\hline$\delta$ & $\begin{array}{r}3.264^{*} \\
(0.21)\end{array}$ & $\begin{array}{r}5.876^{*} \\
(0.34)\end{array}$ & $\begin{array}{r}2.150^{*} \\
(0.12)\end{array}$ & $\begin{array}{r}2.268^{*} \\
(0.13)\end{array}$ & $\begin{array}{r}2.496^{*} \\
(0.14)\end{array}$ & $\begin{array}{r}3.282^{*} \\
(0.21)\end{array}$ & $\begin{array}{r}2.433^{*} \\
(0.14)\end{array}$ & $\begin{array}{r}2.668^{*} \\
(0.16)\end{array}$ \\
\hline AIC & -1198.671 & -1746.093 & -660.372 & -677.892 & -739.670 & -1187.867 & -796.358 & -918.379 \\
\hline
\end{tabular}


Panel B: Parameter estimates for time-varying copulas.

\begin{tabular}{|c|c|c|c|c|c|c|c|c|}
\hline Copula & France & Germany & Greece & Ireland & Italy & Netherlands & Portugal & Spain \\
\hline \multicolumn{9}{|c|}{ TVP-Gaussian } \\
\hline$\psi_{0}$ & $\begin{array}{r}0.697 \\
(4.02)\end{array}$ & $\begin{array}{r}-0.611 \\
(17.72)\end{array}$ & $\begin{array}{r}-1.198^{*} \\
(0.03)\end{array}$ & $\begin{array}{r}-0.870 \\
(70.06)\end{array}$ & $\begin{array}{c}-1.749 \\
(66.30)\end{array}$ & $\begin{array}{r}-1.050 \\
(22.01)\end{array}$ & $\begin{array}{r}-1.836 \\
(10.09)\end{array}$ & $\begin{array}{r}-1.484 \\
(452.12)\end{array}$ \\
\hline$\psi_{1}$ & $\begin{array}{r}0.111 \\
(0.06)\end{array}$ & $\begin{array}{r}0.422 \\
(1.64)\end{array}$ & $\begin{array}{r}0.309^{*} \\
(0.02)\end{array}$ & $\begin{array}{r}0.443 \\
(5.03)\end{array}$ & $\begin{array}{r}0.078 \\
(2.76)\end{array}$ & $\begin{array}{r}-0.132 \\
(0.33)\end{array}$ & $\begin{array}{c}0.249 \\
(0.27)\end{array}$ & $\begin{array}{l}-0.007 \\
(2.44)\end{array}$ \\
\hline$\psi_{2}$ & $\begin{array}{r}2.796 \\
(4.29)\end{array}$ & $\begin{array}{r}5.000 \\
(16.58)\end{array}$ & $\begin{array}{r}4.087^{*} \\
(0.05)\end{array}$ & $\begin{array}{r}3.658 \\
(106.19)\end{array}$ & $\begin{array}{r}5.000 \\
(73.45)\end{array}$ & $\begin{array}{r}5.000 \\
(23.11)\end{array}$ & $\begin{array}{r}5.000 \\
(11.74)\end{array}$ & $\begin{array}{r}5.000 \\
(501.79)\end{array}$ \\
\hline $\mathrm{AIC}$ & -1064.763 & -1691.338 & -628.580 & -656.319 & -710.828 & -1121.542 & -762.466 & -886.352 \\
\hline \multicolumn{9}{|c|}{ TVP-Student } \\
\hline$\psi_{0}$ & $\begin{array}{c}7.669^{*} \\
(0.93)\end{array}$ & $\begin{array}{r}44.715 \\
(115.39)\end{array}$ & $\begin{array}{r}5.629^{*} \\
(1.03)\end{array}$ & $\begin{array}{c}7.100^{*} \\
(1.09)\end{array}$ & $\begin{array}{r}9.028^{*} \\
(1.94)\end{array}$ & $\begin{array}{r}-0.050 \\
(0.11)\end{array}$ & $\begin{array}{r}6.440 \\
(6.81)\end{array}$ & $\begin{array}{r}7.761 \\
(20.72)\end{array}$ \\
\hline$\psi_{1}$ & $\begin{array}{r}-0.017 \\
(0.02)\end{array}$ & $\begin{array}{r}0.001 \\
(0.00)\end{array}$ & $\begin{array}{l}-0.082 \\
(0.04)\end{array}$ & $\begin{array}{r}-0.051 \\
(0.03)\end{array}$ & $\begin{array}{r}-0.091 \\
(0.09)\end{array}$ & $\begin{array}{l}0.337 \\
(0.28)\end{array}$ & $\begin{array}{r}-0.289 \\
(0.29)\end{array}$ & $\begin{array}{r}-0.031 \\
(0.11)\end{array}$ \\
\hline$\psi_{2}$ & $\begin{array}{r}-3.975^{*} \\
(1.07)\end{array}$ & $\begin{array}{c}-40.634 \\
(117.53)\end{array}$ & $\begin{array}{r}-3.406^{*} \\
(1.20)\end{array}$ & $\begin{array}{r}-5.000^{*} \\
(1.21)\end{array}$ & $\begin{array}{r}-6.974^{*} \\
(2.22)\end{array}$ & $\begin{array}{r}0.934 \\
(1.16)\end{array}$ & $\begin{array}{r}-3.260 \\
(7.34)\end{array}$ & $\begin{array}{r}-5.000 \\
(22.96)\end{array}$ \\
\hline U & $\begin{array}{r}1.951^{*} \\
(0.29)\end{array}$ & $\begin{array}{c}3.508^{*} \\
(1.18)\end{array}$ & $\begin{array}{r}2.344^{*} \\
(0.37)\end{array}$ & $\begin{array}{c}2.084^{*} \\
(0.29)\end{array}$ & $\begin{array}{c}3.822^{*} \\
(0.84)\end{array}$ & $\begin{array}{l}5.032^{*} \\
(2.14)\end{array}$ & $\begin{array}{r}11.020^{*} \\
(5.62)\end{array}$ & $\begin{array}{c}3.518 \\
(2.04)\end{array}$ \\
\hline $\mathrm{AIC}$ & -1269.201 & -1756.383 & -688.087 & -723.210 & -755.618 & -1214.939 & -275.838 & -932.676 \\
\hline $\begin{array}{l}\text { TVP-G } \\
\bar{\omega}\end{array}$ & $\begin{array}{r}1.412^{*} \\
(0.11)\end{array}$ & $\begin{array}{r}1.744^{*} \\
(0.24)\end{array}$ & $\begin{array}{c}1.661^{*} \\
(0.41)\end{array}$ & $\begin{array}{r}1.449^{*} \\
(0.29)\end{array}$ & $\begin{array}{r}1.238^{*} \\
(0.61)\end{array}$ & $\begin{array}{c}1.353^{*} \\
(0.10)\end{array}$ & $\begin{array}{c}1.603^{*} \\
(0.40)\end{array}$ & $\begin{array}{r}1.892 \\
(1.13)\end{array}$ \\
\hline $\bar{\beta}$ & $\begin{array}{r}0.172^{*} \\
(0.01)\end{array}$ & $\begin{array}{c}0.138^{*} \\
(0.02)\end{array}$ & $\begin{array}{l}0.095 \\
(0.09)\end{array}$ & $\begin{array}{r}0.148^{*} \\
(0.05)\end{array}$ & $\begin{array}{l}0.176 \\
(0.12)\end{array}$ & $\begin{array}{r}0.179^{*} \\
(0.01)\end{array}$ & $\begin{array}{r}0.112 \\
(0.08)\end{array}$ & $\begin{array}{r}0.049 \\
(0.21)\end{array}$ \\
\hline $\bar{\alpha}$ & $\begin{array}{r}-4.429^{*} \\
(0.88)\end{array}$ & $\begin{array}{r}-5.000^{*} \\
(1.82)\end{array}$ & $\begin{array}{r}-4.880^{*} \\
(1.48)\end{array}$ & $\begin{array}{r}-4.422^{*} \\
(1.17)\end{array}$ & $\begin{array}{r}-3.295 \\
(2.08)\end{array}$ & $\begin{array}{r}-4.174^{*} \\
(0.84)\end{array}$ & $\begin{array}{r}-4.757^{*} \\
(1.56)\end{array}$ & $\begin{array}{r}-5.000 \\
(4.11)\end{array}$ \\
\hline $\mathrm{AIC}$ & -1275.875 & -1730.546 & -698.930 & -768.086 & -766.341 & -1198.528 & -799.470 & -903.540 \\
\hline \multicolumn{9}{|c|}{ TVP-Rotated Gumbel } \\
\hline $\bar{\omega}$ & $\begin{array}{r}1.320^{*} \\
(0.06)\end{array}$ & $\begin{array}{c}1.702^{*} \\
(0.23)\end{array}$ & $\begin{array}{r}1.316^{*} \\
(0.25)\end{array}$ & $\begin{array}{c}1.457^{*} \\
(0.21)\end{array}$ & $\begin{array}{r}0.773^{*} \\
(0.05)\end{array}$ & $\begin{array}{r}1.264^{*} \\
(0.09)\end{array}$ & $\begin{array}{r}1.461^{*} \\
(0.21)\end{array}$ & $\begin{array}{r}1.696^{*} \\
(0.36)\end{array}$ \\
\hline $\bar{\beta}$ & $\begin{array}{r}0.180^{*} \\
(0.01)\end{array}$ & $\begin{array}{r}0.144^{*} \\
(0.01)\end{array}$ & $\begin{array}{r}0.167^{*} \\
(0.05)\end{array}$ & $\begin{array}{r}0.149^{*} \\
(0.03)\end{array}$ & $\begin{array}{r}0.266^{*} \\
(0.01)\end{array}$ & $\begin{array}{r}0.186^{*} \\
(0.01)\end{array}$ & $\begin{array}{r}0.147^{*} \\
(0.04)\end{array}$ & $\begin{array}{l}0.111 \\
(0.06)\end{array}$ \\
\hline $\bar{\alpha}$ & $\begin{array}{r}-3.451^{*} \\
(0.53)\end{array}$ & $\begin{array}{r}-5.000^{*} \\
(2.52)\end{array}$ & $\begin{array}{r}-3.716^{*} \\
(0.99)\end{array}$ & $\begin{array}{r}-4.494^{*} \\
(0.91)\end{array}$ & $\begin{array}{r}-1.664^{*} \\
(0.32)\end{array}$ & $\begin{array}{r}-3.172^{*} \\
(0.67)\end{array}$ & $\begin{array}{r}-4.309^{*} \\
(0.91)\end{array}$ & $\begin{array}{r}-5.000^{*} \\
(1.47)\end{array}$ \\
\hline $\mathrm{AIC}$ & -1335.555 & -1757.076 & -725.442 & -782.999 & -766.521 & -1237.901 & -842.849 & -963.021 \\
\hline
\end{tabular}

Notes. The table reports the ML estimates for the different copula models for the EMU index returns and debt index returns for the European countries indicated. Standard error values (in brackets) and Akaike information criterion (AIC) values adjusted for small-sample bias are provided for the different copula models. The minimum AIC value (in bold) indicates the best copula fit. For the TVP-Gaussian and TVP-Student- $t$ copulas, q in Eq. (3.13) was set to 10 . An asterisk $\left(^{*}\right)$ indicates significance at the $5 \%$ level. 
Table 3.7: Estimates for copula models in the period after crisis onset. European Economic and Monetary Union (EMU) vs. selected countries.

Panel A: Parameter estimates for time-invariant copulas.

\begin{tabular}{|c|c|c|c|c|c|c|c|c|}
\hline Copula & France & Germany & Greece & Ireland & Italy & Netherlands & Portugal & Spain \\
\hline \multicolumn{9}{|c|}{ Gaussian } \\
\hline$\rho$ & $\begin{array}{r}0.679^{*} \\
(0.03)\end{array}$ & $\begin{array}{r}0.994^{*} \\
(0.00)\end{array}$ & $\begin{array}{r}-0.370^{*} \\
(0.06)\end{array}$ & $\begin{array}{r}-0.156^{*} \\
(0.07)\end{array}$ & $\begin{array}{r}-0.182^{*} \\
(0.07)\end{array}$ & $\begin{array}{r}0.914^{*} \\
(0.01)\end{array}$ & $\begin{array}{r}-0.194^{*} \\
(0.07)\end{array}$ & $\begin{array}{r}-0.103 \\
(0.07)\end{array}$ \\
\hline AIC & -94.074 & -686.627 & -20.824 & -1.812 & -3.188 & -279.631 & -3.928 & 0.364 \\
\hline \multicolumn{9}{|c|}{ Student-t } \\
\hline$\rho$ & $\begin{array}{r}0.684^{*} \\
(0.04)\end{array}$ & $\begin{array}{r}0.994^{*} \\
(0.00)\end{array}$ & $\begin{array}{r}-0.362^{*} \\
(0.06)\end{array}$ & $\begin{array}{r}-0.175^{*} \\
(0.09)\end{array}$ & $\begin{array}{r}-0.154 \\
(0.09)\end{array}$ & $\begin{array}{r}0.916^{*} \\
(0.01)\end{array}$ & $\begin{array}{r}-0.192^{*} \\
(0.08)\end{array}$ & $\begin{array}{r}-0.108 \\
(0.08)\end{array}$ \\
\hline$v$ & $\begin{array}{r}10.311 \\
(7.53)\end{array}$ & $\begin{array}{r}17.672 \\
(13.18)\end{array}$ & $\begin{array}{r}59.756 \\
(643.5 \\
2)\end{array}$ & $\begin{array}{c}5.179^{*} \\
(2.43)\end{array}$ & $\begin{array}{r}5.393 \\
(2.86)\end{array}$ & $\begin{array}{r}11.644 \\
(20.36)\end{array}$ & $\begin{array}{r}7.905 \\
(4.69)\end{array}$ & $\begin{array}{r}3.810^{*} \\
(1.57)\end{array}$ \\
\hline AIC & -93.371 & -685.348 & -18.805 & -5.746 & -5.473 & -278.957 & -4.300 & -4.192 \\
\hline \multicolumn{9}{|l|}{ Gumbel } \\
\hline$\delta$ & $\begin{array}{r}1.821^{*} \\
(0.12)\end{array}$ & $\begin{array}{r}13.132^{*} \\
(0.88)\end{array}$ & $\begin{array}{r}1.000^{*} \\
(0.10)\end{array}$ & $\begin{array}{r}1.000^{*} \\
(0.08)\end{array}$ & $\begin{array}{r}1.000^{*} \\
(0.08)\end{array}$ & $\begin{array}{r}3.475^{*} \\
(0.23)\end{array}$ & $\begin{array}{r}1.000^{*} \\
(0.08)\end{array}$ & $\begin{array}{r}1.000 * \\
(0.08)\end{array}$ \\
\hline AIC & -89.144 & -666.026 & 2.026 & 2.026 & 2.026 & -263.297 & 2.026 & 2.026 \\
\hline \multicolumn{9}{|c|}{ Rotated Gumbel } \\
\hline$\delta$ & $\begin{array}{r}1.838^{*} \\
(0.12)\end{array}$ & $\begin{array}{r}13.029^{*} \\
(0.87)\end{array}$ & $\begin{array}{r}1.000^{*} \\
(0.11)\end{array}$ & $\begin{array}{c}1.005^{*} \\
(0.02)\end{array}$ & $\begin{array}{r}1.000^{*} \\
(0.09)\end{array}$ & $\begin{array}{r}3.558^{*} \\
(0.24)\end{array}$ & $\begin{array}{r}1.000^{*} \\
(0.09)\end{array}$ & $\begin{array}{r}1.000^{*} \\
(0.09)\end{array}$ \\
\hline AIC & -88.574 & -666.476 & 2.026 & 1.973 & 2.026 & -267.907 & 2.026 & 2.026 \\
\hline \multicolumn{9}{|l|}{ BB7 } \\
\hline$\theta$ & $\begin{array}{c}1.666^{*} \\
(0.19)\end{array}$ & $\begin{array}{l}5.733^{*} \\
(0.30)\end{array}$ & $\begin{array}{l}1.001^{*} \\
(0.21)\end{array}$ & $\begin{array}{r}1.001^{*} \\
(0.38)\end{array}$ & $\begin{array}{r}1.001^{*} \\
(0.35)\end{array}$ & $\begin{array}{r}3.113^{*} \\
(0.17)\end{array}$ & $\begin{array}{r}1.001^{*} \\
(0.38)\end{array}$ & $\begin{array}{r}1.001^{*} \\
(0.43)\end{array}$ \\
\hline$\delta$ & $\begin{array}{r}0.913^{*} \\
(0.21)\end{array}$ & $\begin{array}{r}10.474^{*} \\
(0.95)\end{array}$ & $\begin{array}{r}0.001 \\
(0.99)\end{array}$ & $\begin{array}{l}0.001 \\
(0.95)\end{array}$ & $\begin{array}{r}0.001 \\
(0.95)\end{array}$ & $\begin{array}{r}2.635^{*} \\
(0.17)\end{array}$ & $\begin{array}{r}0.001 \\
(0.95)\end{array}$ & $\begin{array}{r}0.001 \\
(0.94)\end{array}$ \\
\hline AIC & -88.226 & -588.400 & 4.261 & 4.109 & 4.159 & -258.116 & 4.178 & 4.119 \\
\hline \multicolumn{9}{|c|}{ Plackett } \\
\hline$\theta$ & $\begin{array}{r}9.939^{*} \\
(2.00)\end{array}$ & $\begin{array}{r}665.125^{*} \\
(130.30)\end{array}$ & $\begin{array}{r}0.366^{*} \\
(0.08)\end{array}$ & $\begin{array}{r}0.507^{*} \\
(0.13)\end{array}$ & $\begin{array}{r}0.606^{*} \\
(0.15)\end{array}$ & $\begin{array}{r}48.488^{*} \\
(10.71)\end{array}$ & $\begin{array}{r}0.540^{*} \\
(0.13)\end{array}$ & $\begin{array}{r}0.700^{*} \\
(0.18)\end{array}$ \\
\hline AIC & -94.752 & -648.064 & -15.609 & -4.192 & -2.079 & -270.526 & -4.663 & 0.164 \\
\hline \multicolumn{9}{|l|}{ BB1 } \\
\hline$\theta$ & $\begin{array}{r}0.403^{*} \\
(0.18)\end{array}$ & $\begin{array}{c}0.924^{*} \\
(0.25)\end{array}$ & $\begin{array}{r}0.001 \\
(0.84)\end{array}$ & $\begin{array}{r}0.001 \\
(0.87)\end{array}$ & $\begin{array}{r}0.001 \\
(0.87)\end{array}$ & $\begin{array}{r}0.675^{*} \\
(0.22)\end{array}$ & $\begin{array}{r}0.001 \\
(0.85)\end{array}$ & $\begin{array}{r}0.001 \\
(0.86)\end{array}$ \\
\hline$\delta$ & $\begin{array}{r}1.568^{*} \\
(0.15)\end{array}$ & $\begin{array}{r}9.389^{*} \\
(1.04)\end{array}$ & $\begin{array}{l}1.001 \\
(0.66)\end{array}$ & $\begin{array}{l}1.001 \\
(0.57)\end{array}$ & $\begin{array}{l}1.001 \\
(0.58)\end{array}$ & $\begin{array}{r}2.720^{*} \\
(0.28)\end{array}$ & $\begin{array}{l}1.001 \\
(0.64)\end{array}$ & $\begin{array}{r}1.001 \\
(0.59)\end{array}$ \\
\hline $\mathrm{AIC}$ & -92.345 & -686.060 & 4.327 & 4.135 & 4.188 & -274.670 & 4.215 & 4.132 \\
\hline
\end{tabular}


Panel B: Parameter estimates for time-varying copulas.

\begin{tabular}{|c|c|c|c|c|c|c|c|c|}
\hline Copula & France & Germany & Greece & Ireland & Italy & Netherlands & Portugal & Spain \\
\hline \multicolumn{9}{|c|}{ TVP-Gaussian } \\
\hline$\psi_{0}$ & $\begin{array}{r}2.916 \\
(41.03)\end{array}$ & $\begin{array}{r}5.000 \\
(83.18)\end{array}$ & $\begin{array}{r}-0.845 \\
(0.49)\end{array}$ & $\begin{array}{c}-0.185 \\
(0.18)\end{array}$ & $\begin{array}{l}-0.290 \\
(2.50)\end{array}$ & $\begin{array}{r}5.000 \\
(8.76)\end{array}$ & $\begin{array}{r}-0.388 \\
(1.36)\end{array}$ & $\begin{array}{r}-0.003 \\
(0.27)\end{array}$ \\
\hline$\psi_{1}$ & $\begin{array}{r}0.116 \\
(5.85)\end{array}$ & $\begin{array}{r}0.015 \\
(25.41)\end{array}$ & $\begin{array}{r}-0.372 \\
(0.48)\end{array}$ & $\begin{array}{c}-0.248 \\
(0.22)\end{array}$ & $\begin{array}{r}1.130 \\
(0.86)\end{array}$ & $\begin{array}{c}-0.284 \\
(0.38)\end{array}$ & $\begin{array}{r}-0.148 \\
(0.47)\end{array}$ & $\begin{array}{r}1.201^{*} \\
(0.33)\end{array}$ \\
\hline$\psi_{2}$ & $\begin{array}{r}-2.000 \\
(63.09)\end{array}$ & $\begin{array}{r}1.000 \\
(56.93)\end{array}$ & $\begin{array}{r}0.126 \\
(1.02)\end{array}$ & $\begin{array}{l}1.160 \\
(0.77)\end{array}$ & $\begin{array}{r}-2.000 \\
(5.33)\end{array}$ & $\begin{array}{r}-1.761 \\
(9.33)\end{array}$ & $\begin{array}{r}0.116 \\
(6.93)\end{array}$ & $\begin{array}{r}-2.000 * \\
(0.05)\end{array}$ \\
\hline $\mathrm{AIC}$ & -90.021 & -682.559 & -17.566 & 0.373 & -9.741 & -276.408 & -0.009 & -4.988 \\
\hline \multicolumn{9}{|c|}{ TVP-Student } \\
\hline$\psi_{0}$ & $\begin{array}{l}1.595 \\
(2.07)\end{array}$ & $\begin{array}{c}106.808 \\
(161.24)\end{array}$ & $\begin{array}{r}-0.838^{*} \\
(0.26)\end{array}$ & $\begin{array}{l}-0.021 \\
(0.03)\end{array}$ & $\begin{array}{r}-0.044 \\
(0.04)\end{array}$ & $\begin{array}{r}6.440 \\
(6.81)\end{array}$ & $\begin{array}{r}-0.583 \\
(1.24)\end{array}$ & $\begin{array}{r}-0.070 \\
(0.12)\end{array}$ \\
\hline$\psi_{1}$ & $\begin{array}{r}-0.195 \\
(0.27)\end{array}$ & $\begin{array}{r}0.155 \\
(0.32)\end{array}$ & $\begin{array}{r}-0.362 \\
(0.29)\end{array}$ & $\begin{array}{r}-0.110^{*} \\
(0.06)\end{array}$ & $\begin{array}{r}0.181 \\
(0.14)\end{array}$ & $\begin{array}{c}-0.289 \\
(0.29)\end{array}$ & $\begin{array}{r}-0.099 \\
(0.37)\end{array}$ & $\begin{array}{r}0.299 \\
(0.23)\end{array}$ \\
\hline$\psi_{2}$ & $\begin{array}{r}0.363 \\
(2.71)\end{array}$ & $\begin{array}{c}-101.562 \\
(161.13)\end{array}$ & $\begin{array}{l}0.132 \\
(0.66)\end{array}$ & $\begin{array}{r}2.159^{*} \\
(0.10)\end{array}$ & $\begin{array}{l}1.600^{*} \\
(0.48)\end{array}$ & $\begin{array}{r}-3.260 \\
(7.34)\end{array}$ & $\begin{array}{r}-0.817 \\
(6.21)\end{array}$ & $\begin{array}{l}0.807 \\
(0.93)\end{array}$ \\
\hline U & $\begin{array}{r}6.862 \\
(5.16)\end{array}$ & $\begin{array}{r}17.439 \\
(19.73)\end{array}$ & $\begin{array}{r}100.000^{*} \\
(0.00)\end{array}$ & $\begin{array}{r}5.309^{*} \\
(1.48)\end{array}$ & $\begin{array}{c}4.833^{*} \\
(1.93)\end{array}$ & $\begin{array}{r}11.020^{*} \\
(5.62)\end{array}$ & $\begin{array}{r}7.982 \\
(5.82)\end{array}$ & $\begin{array}{r}4.420^{*} \\
(1.88)\end{array}$ \\
\hline $\mathrm{AIC}$ & -89.577 & -681.335 & -15.464 & -8.163 & -15.034 & -275.838 & -0.194 & -6.759 \\
\hline \multicolumn{9}{|c|}{ TVP-Gumbel } \\
\hline $\bar{\omega}$ & $\begin{array}{r}2.167^{*} \\
(0.62)\end{array}$ & $\begin{array}{l}1.984^{*} \\
(0.23)\end{array}$ & $\begin{array}{r}0.000 \\
(1.00)\end{array}$ & $\begin{array}{r}0.000 \\
(1.00)\end{array}$ & $\begin{array}{r}0.000 \\
(1.00)\end{array}$ & $\begin{array}{c}0.771^{*} \\
(0.21)\end{array}$ & $\begin{array}{r}0.000 \\
(1.00)\end{array}$ & $\begin{array}{r}0.000 \\
(1.00)\end{array}$ \\
\hline $\bar{\beta}$ & $\begin{array}{c}-0.154 \\
(0.21)\end{array}$ & $\begin{array}{r}0.123^{*} \\
(0.02)\end{array}$ & $\begin{array}{r}0.000 \\
(1.00)\end{array}$ & $\begin{array}{r}0.000 \\
(1.00)\end{array}$ & $\begin{array}{r}0.000 \\
(1.00)\end{array}$ & $\begin{array}{r}0.258^{*} \\
(0.04)\end{array}$ & $\begin{array}{r}0.000 \\
(1.00)\end{array}$ & $\begin{array}{r}0.000 \\
(1.00)\end{array}$ \\
\hline $\bar{\alpha}$ & $\begin{array}{r}-5.000^{*} \\
(1.25)\end{array}$ & $\begin{array}{r}-4.619 \\
(2.46)\end{array}$ & $\begin{array}{r}0.000 \\
(1.00)\end{array}$ & $\begin{array}{r}0.000 \\
(1.00)\end{array}$ & $\begin{array}{r}0.000 \\
(1.00)\end{array}$ & $\begin{array}{r}-1.107 \\
(0.76)\end{array}$ & $\begin{array}{r}0.000 \\
(1.00)\end{array}$ & $\begin{array}{r}0.000 \\
(1.00)\end{array}$ \\
\hline $\mathrm{AIC}$ & -112.068 & -663.314 & 6.175 & 6.163 & 6.167 & -263.027 & 6.169 & 6.163 \\
\hline \multicolumn{9}{|c|}{ TVP-Rotated Gumbel } \\
\hline $\bar{\omega}$ & $\begin{array}{l}0.847 \\
(0.48)\end{array}$ & $\begin{array}{r}2.920 \\
(1.82)\end{array}$ & $\begin{array}{r}0.000 \\
(1.00)\end{array}$ & $\begin{array}{r}-1.399^{*} \\
(0.53)\end{array}$ & $\begin{array}{r}0.000 \\
(1.00)\end{array}$ & $\begin{array}{c}0.633^{*} \\
(0.10)\end{array}$ & $\begin{array}{r}0.000 \\
(1.00)\end{array}$ & $\begin{array}{r}0.000 \\
(1.00)\end{array}$ \\
\hline $\bar{\beta}$ & $\begin{array}{r}0.252 \\
(0.13)\end{array}$ & $\begin{array}{r}0.052 \\
(0.13)\end{array}$ & $\begin{array}{r}0.000 \\
(1.00)\end{array}$ & $\begin{array}{l}1.001 \\
(0.62)\end{array}$ & $\begin{array}{r}0.000 \\
(1.00)\end{array}$ & $\begin{array}{r}0.286^{*} \\
(0.02)\end{array}$ & $\begin{array}{r}0.000 \\
(1.00)\end{array}$ & $\begin{array}{r}0.000 \\
(1.00)\end{array}$ \\
\hline $\bar{\alpha}$ & $\begin{array}{r}-2.317 \\
(1.24)\end{array}$ & $\begin{array}{r}-5.000 \\
(12.52)\end{array}$ & $\begin{array}{r}0.000 \\
(1.00)\end{array}$ & $\begin{array}{l}1.248 \\
(0.95)\end{array}$ & $\begin{array}{r}0.000 \\
(1.00)\end{array}$ & $\begin{array}{r}-0.655 \\
(0.39)\end{array}$ & $\begin{array}{r}0.000 \\
(1.00)\end{array}$ & $\begin{array}{r}0.000 \\
(1.00)\end{array}$ \\
\hline $\mathrm{AIC}$ & 1.000 & -662.873 & 6.173 & 4.807 & 6.161 & -267.848 & 6.161 & 6.160 \\
\hline
\end{tabular}

Notes. See Table 3.6 notes. 
Table 3.8: Estimates for the copula models in the period before crisis onset. Greece vs. country.

Panel A: Parameter estimates for time-invariant copulas.

\begin{tabular}{|c|c|c|c|c|c|c|c|}
\hline Copula & France & Germany & Ireland & Italy & Netherlands & Portugal & Spain \\
\hline \multicolumn{8}{|c|}{ Gaussian } \\
\hline$\rho$ & $\begin{array}{r}0.856^{*} \\
(0.01)\end{array}$ & $\begin{array}{r}0.834^{*} \\
(0.01)\end{array}$ & $\begin{array}{r}0.916^{*} \\
(0.01)\end{array}$ & $\begin{array}{c}0.932^{*} \\
(0.00)\end{array}$ & $\begin{array}{r}0.867^{*} \\
(0.01)\end{array}$ & $\begin{array}{c}0.944^{*} \\
(0.00)\end{array}$ & $\begin{array}{r}0.915^{*} \\
(0.01)\end{array}$ \\
\hline AIC & -671.901 & -602.133 & -932.624 & -1035.026 & -707.130 & -1128.174 & -925.587 \\
\hline \multicolumn{8}{|c|}{ Student-t } \\
\hline$\rho$ & $\begin{array}{r}0.878^{*} \\
(0.01)\end{array}$ & $\begin{array}{r}0.857^{*} \\
(0.01)\end{array}$ & $\begin{array}{r}0.940^{*} \\
(0.01)\end{array}$ & $\begin{array}{r}0.949^{*} \\
(0.01)\end{array}$ & $\begin{array}{r}0.880^{*} \\
(0.01)\end{array}$ & $\begin{array}{r}0.952^{*} \\
(0.01)\end{array}$ & $\begin{array}{r}0.931^{*} \\
(0.01)\end{array}$ \\
\hline$v$ & $\begin{array}{r}2.318^{*} \\
(0.43)\end{array}$ & $\begin{array}{r}3.061^{*} \\
(0.57)\end{array}$ & $\begin{array}{c}1.343^{*} \\
(0.16)\end{array}$ & $\begin{array}{r}1.521^{*} \\
(0.19)\end{array}$ & $\begin{array}{r}2.287^{*} \\
(0.34)\end{array}$ & $\begin{array}{r}2.489^{*} \\
(1.01)\end{array}$ & $\begin{array}{r}2.094^{*} \\
(0.29)\end{array}$ \\
\hline $\mathrm{AIC}$ & -772.265 & -682.688 & -1147.700 & -1200.938 & -784.636 & -1213.367 & -1044.899 \\
\hline \multicolumn{8}{|l|}{ Gumbel } \\
\hline$\delta$ & $\begin{array}{r}3.060^{*} \\
(0.12)\end{array}$ & $\begin{array}{r}2.743^{*} \\
(0.10)\end{array}$ & $\begin{array}{l}4.266^{*} \\
(0.17)\end{array}$ & $\begin{array}{r}4.647^{*} \\
(0.18)\end{array}$ & $\begin{array}{r}3.065^{*} \\
(0.12)\end{array}$ & $\begin{array}{c}4.812^{*} \\
(0.18)\end{array}$ & $\begin{array}{r}3.960^{*} \\
(0.15)\end{array}$ \\
\hline $\mathrm{AIC}$ & -704.236 & -616.276 & -982.597 & -1073.074 & -710.077 & -1125.291 & -932.318 \\
\hline \multicolumn{8}{|c|}{ Rotated Gumbel } \\
\hline$\delta$ & $\begin{array}{r}3.035^{*} \\
(0.11)\end{array}$ & $\begin{array}{r}2.798^{*} \\
(0.10)\end{array}$ & $\begin{array}{l}4.496^{*} \\
(0.17)\end{array}$ & $\begin{array}{r}4.766^{*} \\
(0.18)\end{array}$ & $\begin{array}{r}3.148^{*} \\
(0.12)\end{array}$ & $\begin{array}{c}4.971^{*} \\
(0.18)\end{array}$ & $\begin{array}{r}4.200^{*} \\
(0.16)\end{array}$ \\
\hline $\mathrm{AIC}$ & -710.350 & -640.877 & -1049.016 & -1108.588 & -745.980 & -1165.662 & -1002.412 \\
\hline \multicolumn{8}{|l|}{ BB7 } \\
\hline$\theta$ & $\begin{array}{r}2.836 \\
(3.81)\end{array}$ & $\begin{array}{r}2.399^{*} \\
(0.23)\end{array}$ & $\begin{array}{l}3.639^{*} \\
(0.25)\end{array}$ & $\begin{array}{c}4.126^{*} \\
(0.01)\end{array}$ & $\begin{array}{r}2.692^{*} \\
(0.22)\end{array}$ & $\begin{array}{r}5.275^{*} \\
(0.04)\end{array}$ & $\begin{array}{r}3.353^{*} \\
(0.17)\end{array}$ \\
\hline$\delta$ & $\begin{array}{r}2.143^{*} \\
(0.29)\end{array}$ & $\begin{array}{c}2.029^{*} \\
(0.14)\end{array}$ & $\begin{array}{c}4.483^{*} \\
(0.41)\end{array}$ & $\begin{array}{r}4.269 * \\
(0.03)\end{array}$ & $\begin{array}{r}2.553^{*} \\
(0.17)\end{array}$ & $\begin{array}{c}5.074^{*} \\
(0.00)\end{array}$ & $\begin{array}{r}4.036^{*} \\
(0.23)\end{array}$ \\
\hline AIC & -712.089 & -630.231 & -1022.834 & -1068.123 & -740.602 & -1126.389 & -969.510 \\
\hline \multicolumn{8}{|c|}{ Plackett } \\
\hline$\theta$ & $\begin{array}{r}43.107^{*} \\
(6.44)\end{array}$ & $\begin{array}{r}31.535^{*} \\
(3.10)\end{array}$ & $\begin{array}{r}125.294^{*} \\
(6.78)\end{array}$ & $\begin{array}{r}143.040^{*} \\
(14.70)\end{array}$ & $\begin{array}{r}44.410^{*} \\
(3.60)\end{array}$ & $\begin{array}{r}117.270^{*} \\
(11.82)\end{array}$ & $\begin{array}{r}82.852^{*} \\
(5.60)\end{array}$ \\
\hline AIC & -763.799 & -666.266 & -1137.193 & -1209.029 & -768.939 & -1190.713 & -1030.318 \\
\hline \multicolumn{8}{|l|}{ BB1 } \\
\hline$\theta$ & $\begin{array}{r}0.609^{*} \\
(0.12)\end{array}$ & $\begin{array}{c}0.674^{*} \\
(0.13)\end{array}$ & $\begin{array}{c}1.093^{*} \\
(0.16)\end{array}$ & $\begin{array}{c}0.861^{*} \\
(0.12)\end{array}$ & $\begin{array}{r}0.806^{*} \\
(0.14)\end{array}$ & $\begin{array}{r}0.925^{*} \\
(0.15)\end{array}$ & $\begin{array}{r}1.068^{*} \\
(0.23)\end{array}$ \\
\hline$\delta$ & $\begin{array}{r}2.425^{*} \\
(0.14)\end{array}$ & $\begin{array}{r}2.140^{*} \\
(0.13)\end{array}$ & $\begin{array}{r}2.942^{*} \\
(0.19)\end{array}$ & $\begin{array}{c}3.409^{*} \\
(0.20)\end{array}$ & $\begin{array}{r}2.290^{*} \\
(0.14)\end{array}$ & $\begin{array}{r}3.474^{*} \\
(0.22)\end{array}$ & $\begin{array}{r}2.750^{*} \\
(0.22)\end{array}$ \\
\hline $\mathrm{AIC}$ & -740.509 & -656.994 & -1061.755 & -1130.593 & -763.160 & -1190.198 & -1011.073 \\
\hline
\end{tabular}


Panel B: Parameter estimates for time-varying copulas.

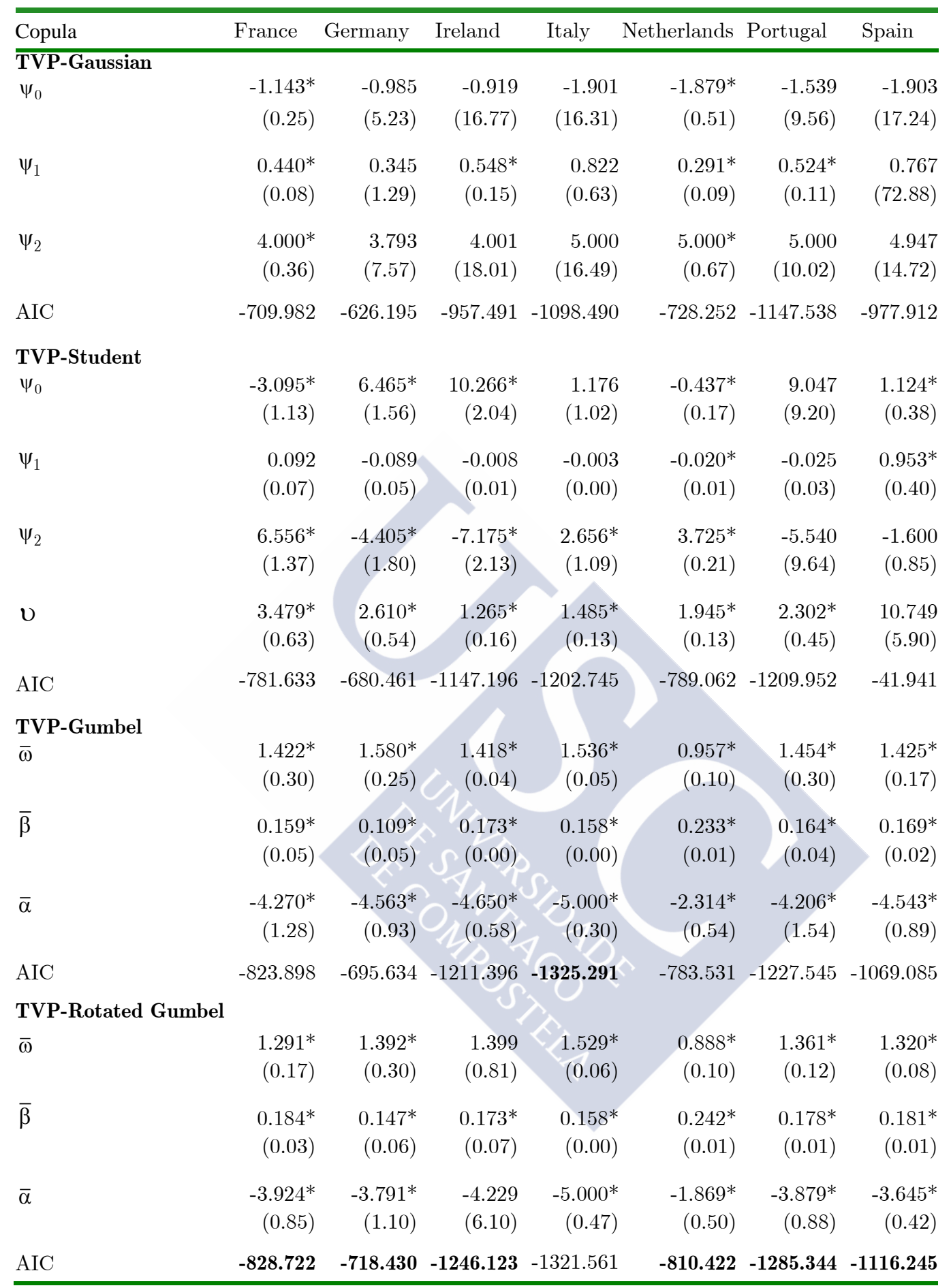

Notes. See Table 3.6 notes. 
Table 3.9: Estimates for the copula models in the period after crisis onset. Greece vs. country.

Panel A: Parameter estimates for time-invariant copulas.

\begin{tabular}{|c|c|c|c|c|c|c|c|}
\hline Copula & France & Germany & Ireland & Italy & Netherlands & Portugal & Spain \\
\hline \multicolumn{8}{|c|}{ Gaussian } \\
\hline$\rho$ & $\begin{array}{r}-0.061 \\
(0.08)\end{array}$ & $\begin{array}{r}-0.377^{*} \\
(0.06)\end{array}$ & $\begin{array}{r}0.473^{*} \\
(0.06)\end{array}$ & $\begin{array}{r}0.417^{*} \\
(0.06)\end{array}$ & $\begin{array}{r}-0.264^{*} \\
(0.07)\end{array}$ & $\begin{array}{r}0.470^{*} \\
(0.06)\end{array}$ & $\begin{array}{r}0.400^{*} \\
(0.06)\end{array}$ \\
\hline AIC & 1.432 & -21.754 & -37.308 & -27.601 & -9.276 & -36.665 & -24.910 \\
\hline \multicolumn{8}{|c|}{ Student-t } \\
\hline$\rho$ & $\begin{array}{r}-0.059 \\
(0.20)\end{array}$ & $\begin{array}{r}-0.368^{*} \\
(0.08)\end{array}$ & $\begin{array}{r}0.449^{*} \\
(0.04)\end{array}$ & $\begin{array}{c}0.384^{*} \\
(0.05)\end{array}$ & $\begin{array}{r}-0.258^{*} \\
(0.07)\end{array}$ & $\begin{array}{r}0.440^{*} \\
(0.06)\end{array}$ & $\begin{array}{r}0.377^{*} \\
(0.06)\end{array}$ \\
\hline$v$ & $\begin{array}{r}500.000 \\
(1377.3 \\
5)\end{array}$ & $\begin{array}{c}37.902 \\
(36.14)\end{array}$ & $\begin{array}{r}100.000 \\
(81.42)\end{array}$ & $\begin{array}{r}32.619 \\
(89.61)\end{array}$ & $\begin{array}{r}500.000 \\
(1758.78)\end{array}$ & $\begin{array}{l}8.075 \\
(6.33)\end{array}$ & $\begin{array}{r}22.329 \\
(38.08)\end{array}$ \\
\hline $\mathrm{AIC}$ & 3.629 & -19.772 & -35.395 & -25.960 & -7.196 & -36.803 & -23.631 \\
\hline \multicolumn{8}{|c|}{ Gumbel } \\
\hline$\delta$ & $\begin{array}{r}1.000^{*} \\
(0.08)\end{array}$ & $\begin{array}{c}1.000^{*} \\
(0.10)\end{array}$ & $\begin{array}{c}1.344^{*} \\
(0.08)\end{array}$ & $\begin{array}{c}1.269^{*} \\
(0.07)\end{array}$ & $\begin{array}{l}1.000^{*} \\
(0.09)\end{array}$ & $\begin{array}{r}1.372^{*} \\
(0.08)\end{array}$ & $\begin{array}{r}1.270^{*} \\
(0.07)\end{array}$ \\
\hline $\mathrm{AIC}$ & 2.026 & 2.026 & -34.138 & -25.111 & 2.026 & -40.190 & -23.783 \\
\hline \multicolumn{8}{|c|}{ Rotated Gumbel } \\
\hline$\delta$ & $\begin{array}{r}1.000^{*} \\
(0.10)\end{array}$ & $\begin{array}{r}1.000^{*} \\
(0.11)\end{array}$ & $\begin{array}{c}1.350^{*} \\
(0.08)\end{array}$ & $\begin{array}{l}1.280^{*} \\
(0.07)\end{array}$ & $\begin{array}{l}1.000^{*} \\
(0.10)\end{array}$ & $\begin{array}{r}1.367^{*} \\
(0.08)\end{array}$ & $\begin{array}{r}1.269^{*} \\
(0.07)\end{array}$ \\
\hline $\mathrm{AIC}$ & 2.026 & 2.026 & -28.568 & -22.014 & 2.026 & -30.844 & -18.434 \\
\hline \multicolumn{8}{|l|}{ BB7 } \\
\hline$\theta$ & $\begin{array}{r}1.001^{*} \\
(0.46)\end{array}$ & $\begin{array}{c}1.001^{*} \\
(0.20)\end{array}$ & $\begin{array}{c}1.294^{*} \\
(0.13)\end{array}$ & $\begin{array}{r}1.229^{*} \\
(0.09)\end{array}$ & $\begin{array}{c}1.001^{*} \\
(0.29)\end{array}$ & $\begin{array}{r}1.402^{*} \\
(0.12)\end{array}$ & $\begin{array}{r}1.235^{*} \\
(0.11)\end{array}$ \\
\hline$\delta$ & $\begin{array}{l}0.001 \\
(0.93)\end{array}$ & $\begin{array}{l}0.001 \\
(0.99)\end{array}$ & $\begin{array}{c}0.307^{*} \\
(0.11)\end{array}$ & $\begin{array}{c}0.299^{*} \\
(0.11)\end{array}$ & $\begin{array}{r}0.001 \\
(0.97)\end{array}$ & $\begin{array}{r}0.252^{*} \\
(0.12)\end{array}$ & $\begin{array}{r}0.261^{*} \\
(0.12)\end{array}$ \\
\hline $\mathrm{AIC}$ & 4.172 & 4.261 & -31.717 & -25.525 & 4.235 & -38.020 & -21.565 \\
\hline \multicolumn{8}{|c|}{ Plackett } \\
\hline$\theta$ & $\begin{array}{r}0.925^{*} \\
(0.19)\end{array}$ & $\begin{array}{r}0.353^{*} \\
(0.08)\end{array}$ & $\begin{array}{c}4.171^{*} \\
(0.90)\end{array}$ & $\begin{array}{c}3.110^{*} \\
(0.69)\end{array}$ & $\begin{array}{r}0.523^{*} \\
(0.12)\end{array}$ & $\begin{array}{r}4.276^{*} \\
(0.94)\end{array}$ & $\begin{array}{r}3.170^{*} \\
(0.68)\end{array}$ \\
\hline $\mathrm{AIC}$ & 1.887 & -16.633 & -33.880 & -20.940 & -5.663 & -34.341 & -23.500 \\
\hline \multicolumn{8}{|l|}{ BB1 } \\
\hline$\theta$ & $\begin{array}{r}0.001 \\
(0.85)\end{array}$ & $\begin{array}{l}0.001 \\
(0.84)\end{array}$ & $\begin{array}{r}0.149 \\
(0.12)\end{array}$ & $\begin{array}{r}0.187 \\
(0.13)\end{array}$ & $\begin{array}{l}0.001 \\
(0.84)\end{array}$ & $\begin{array}{l}0.092 \\
(0.12)\end{array}$ & $\begin{array}{r}0.131 \\
(0.12)\end{array}$ \\
\hline$\delta$ & $\begin{array}{l}1.001 \\
(0.62)\end{array}$ & $\begin{array}{l}1.001 \\
(0.66)\end{array}$ & $\begin{array}{r}1.267^{*} \\
(0.09)\end{array}$ & $\begin{array}{r}1.188^{*} \\
(0.08)\end{array}$ & $\begin{array}{l}1.001 \\
(0.64)\end{array}$ & $\begin{array}{r}1.325^{*} \\
(0.10)\end{array}$ & $\begin{array}{r}1.211^{*} \\
(0.08)\end{array}$ \\
\hline $\mathrm{AIC}$ & 4.188 & 4.327 & -33.753 & -25.888 & 4.284 & -38.826 & -22.940 \\
\hline
\end{tabular}


Panel B: Parameter estimates for time-varying copulas.

\begin{tabular}{|c|c|c|c|c|c|c|c|}
\hline Copula & France & Germany & Ireland & Italy & Netherlands & Portugal & Spain \\
\hline \multicolumn{8}{|c|}{ TVP-Gaussian } \\
\hline$\psi_{0}$ & $\begin{array}{r}-0.081 \\
(0.13)\end{array}$ & $\begin{array}{r}-0.805 \\
(0.88)\end{array}$ & $\begin{array}{r}1.725^{*} \\
(0.81)\end{array}$ & $\begin{array}{r}0.336 \\
(0.71)\end{array}$ & $\begin{array}{r}-0.891 \\
(0.54)\end{array}$ & $\begin{array}{r}1.077 \\
(0.81)\end{array}$ & $\begin{array}{r}0.175 \\
(0.30)\end{array}$ \\
\hline$\psi_{1}$ & $\begin{array}{r}-0.367 \\
(0.56)\end{array}$ & $\begin{array}{r}-0.335 \\
(0.47)\end{array}$ & $\begin{array}{r}0.244 \\
(0.29)\end{array}$ & $\begin{array}{r}-0.062 \\
(0.17)\end{array}$ & $\begin{array}{l}-0.751 \\
(0.67)\end{array}$ & $\begin{array}{r}1.530 \\
(1.25)\end{array}$ & $\begin{array}{r}-0.144 \\
(0.19)\end{array}$ \\
\hline$\psi_{2}$ & $\begin{array}{r}0.719 \\
(1.62)\end{array}$ & $\begin{array}{r}0.246 \\
(2.06)\end{array}$ & $\begin{array}{r}-1.946 \\
(1.56)\end{array}$ & $\begin{array}{r}1.314 \\
(1.72)\end{array}$ & $\begin{array}{r}-0.604 \\
(1.55)\end{array}$ & $\begin{array}{l}-2.000 \\
(1.34)\end{array}$ & $\begin{array}{r}1.792^{*} \\
(0.63)\end{array}$ \\
\hline $\mathrm{AIC}$ & 4.684 & -18.442 & -33.568 & -23.856 & -6.916 & -44.739 & -22.290 \\
\hline \multicolumn{8}{|c|}{ TVP-Student } \\
\hline$\psi_{0}$ & $\begin{array}{r}-0.110 \\
(0.34)\end{array}$ & $\begin{array}{r}-0.807 \\
(0.85)\end{array}$ & $\begin{array}{r}1.836^{*} \\
(0.58)\end{array}$ & $\begin{array}{r}0.321 \\
(0.65)\end{array}$ & $\begin{array}{r}-0.891 \\
(1.24)\end{array}$ & $\begin{array}{r}1.124^{*} \\
(0.38)\end{array}$ & $\begin{array}{r}1.723^{*} \\
(0.47)\end{array}$ \\
\hline$\psi_{1}$ & $\begin{array}{r}0.150 \\
(0.35)\end{array}$ & $\begin{array}{r}-0.325 \\
(0.41)\end{array}$ & $\begin{array}{r}0.595^{*} \\
(0.15)\end{array}$ & $\begin{array}{r}-0.053 \\
(0.15)\end{array}$ & $\begin{array}{r}-0.753 \\
(0.59)\end{array}$ & $\begin{array}{r}0.953^{*} \\
(0.40)\end{array}$ & $\begin{array}{r}-0.322 \\
(0.49)\end{array}$ \\
\hline$\psi_{2}$ & $\begin{array}{r}-2.153^{*} \\
(0.05)\end{array}$ & $\begin{array}{l}0.225 \\
(2.10)\end{array}$ & $\begin{array}{r}-2.583^{*} \\
(0.50)\end{array}$ & $\begin{array}{r}1.338 \\
(1.64)\end{array}$ & $\begin{array}{r}-0.601 \\
(5.29)\end{array}$ & $\begin{array}{r}-1.600 \\
(0.85)\end{array}$ & $\begin{array}{r}-2.000^{*} \\
(0.65)\end{array}$ \\
\hline$v$ & $\begin{array}{r}499.046 \\
(826.55)\end{array}$ & $\begin{array}{r}53.091 \\
(307.10)\end{array}$ & $\begin{array}{r}51.820 \\
(182.00)\end{array}$ & $\begin{array}{r}35.602 \\
(101.05)\end{array}$ & $\begin{array}{r}500.000 \\
(1772.87)\end{array}$ & $\begin{array}{r}10.749 \\
(5.90)\end{array}$ & $\begin{array}{r}25.178 \\
(39.80)\end{array}$ \\
\hline AIC & 7.628 & -16.369 & -32.884 & -21.919 & -4.767 & -41.941 & -19.824 \\
\hline $\begin{array}{l}\text { TVP-G } \\
\bar{\omega}\end{array}$ & $\begin{array}{r}0.000 \\
(1.00)\end{array}$ & $\begin{array}{r}0.000 \\
(1.00)\end{array}$ & $\begin{array}{l}2.251^{*} \\
(0.32)\end{array}$ & $\begin{array}{r}1.283 \\
(0.79)\end{array}$ & $\begin{array}{r}0.000 \\
(1.00)\end{array}$ & $\begin{array}{r}0.757 \\
(0.99)\end{array}$ & $\begin{array}{r}1.079 \\
(0.64)\end{array}$ \\
\hline $\bar{\beta}$ & $\begin{array}{r}0.000 \\
(1.00)\end{array}$ & $\begin{array}{r}0.000 \\
(1.00)\end{array}$ & $\begin{array}{r}-0.750^{*} \\
(0.10)\end{array}$ & $\begin{array}{r}-0.182 \\
(0.45)\end{array}$ & $\begin{array}{r}0.000 \\
(1.00)\end{array}$ & $\begin{array}{r}0.241 \\
(0.40)\end{array}$ & $\begin{array}{r}-0.169 \\
(0.43)\end{array}$ \\
\hline $\bar{\alpha}$ & $\begin{array}{r}0.000 \\
(1.00)\end{array}$ & $\begin{array}{r}0.000 \\
(1.00)\end{array}$ & $\begin{array}{l}-2.665 \\
(1.64)\end{array}$ & $\begin{array}{r}-1.947 \\
(1.15)\end{array}$ & $\begin{array}{r}0.000 \\
(1.00)\end{array}$ & $\begin{array}{l}-1.904 \\
(1.75)\end{array}$ & $\begin{array}{r}-1.301 \\
(0.92)\end{array}$ \\
\hline $\mathrm{AIC}$ & 6.167 & 6.174 & -32.556 & -24.977 & 6.172 & -47.444 & -21.348 \\
\hline \multicolumn{8}{|c|}{ TVP-Rotated Gumbel } \\
\hline $\bar{\omega}$ & $\begin{array}{r}0.000 \\
(1.00)\end{array}$ & $\begin{array}{r}0.000 \\
(1.00)\end{array}$ & $\begin{array}{r}0.515 \\
(1.04)\end{array}$ & $\begin{array}{r}1.018 \\
(1.27)\end{array}$ & $\begin{array}{r}0.000 \\
(1.00)\end{array}$ & $\begin{array}{r}0.622^{*} \\
(0.24)\end{array}$ & $\begin{array}{r}0.669 \\
(1.67)\end{array}$ \\
\hline $\bar{\beta}$ & $\begin{array}{r}0.000 \\
(1.00)\end{array}$ & $\begin{array}{r}0.000 \\
(1.00)\end{array}$ & $\begin{array}{r}0.300 \\
(0.49)\end{array}$ & $\begin{array}{r}-0.290 \\
(0.94)\end{array}$ & $\begin{array}{r}0.000 \\
(1.00)\end{array}$ & $\begin{array}{r}0.316^{*} \\
(0.08)\end{array}$ & $\begin{array}{r}0.012 \\
(1.19)\end{array}$ \\
\hline $\bar{\alpha}$ & $\begin{array}{r}0.000 \\
(1.00)\end{array}$ & $\begin{array}{r}0.000 \\
(1.00)\end{array}$ & $\begin{array}{r}-1.396 \\
(1.70)\end{array}$ & $\begin{array}{r}-0.430 \\
(1.11)\end{array}$ & $\begin{array}{r}0.000 \\
(1.00)\end{array}$ & $\begin{array}{r}-1.871^{*} \\
(0.66)\end{array}$ & $\begin{array}{r}-0.647 \\
(1.13)\end{array}$ \\
\hline $\mathrm{AIC}$ & 6.164 & 6.173 & -28.235 & -18.030 & 6.170 & -38.570 & -14.679 \\
\hline
\end{tabular}

Notes. See Table 3.6 notes. 


\subsubsection{CoVaR results}

Using the best copula fit for the pre- and post-onset periods, and following the twostep procedure described above, we computed the CoVaR at the $99 \%$ confidence level $(\beta=0.01)$ for the European sovereign debt market, conditional on the VaR for the sovereign debt returns of different countries at the $99 \%$ confidence level $(\alpha=0.01){ }^{6}$

Figure 3.4 depicts the results for the $\mathrm{CoVaR}$ and ${ }_{\Delta} \mathrm{CoVaR}$ (in percentage) dynamics in the pre- and post-onset periods (delimited by a vertical line). For each figure that represents the systemic risk of each country, we also included information on the CoVaR, as computed using the methodology proposed by Girardi and Ergün (2013). Thus, taking a bivariate dynamic conditional correlation GARCH model with a bivariate Student-t density for the standardized residuals of the marginal models in Eqs. (3.8)-(3.10), and taking the value of $\mathrm{VaR}^{7}$ for the market under distress, we numerically solved Eq. (3.4). We could thus visualize the differences between the copula approach and the approach used by Girardi and Ergün (2013) to compute the CoVaR and so assess the impact of copula modelling on the CoVaR values.

Figure 3.4 indicates that, in the pre-onset period, all European debt markets experienced a similar trend in terms of systemic risk, with the size of this risk very similar across debt markets. Furthermore, the CoVaR measure clearly captured the impact of the dot-com bubble and the subprime crisis on systemic risk with significant reductions in the CoVaR values. The $\triangle \mathrm{CoVaR}$ dynamics were similar, displaying significant increases around the time of the dot-com bubble and recent global financial crises. Table 3.10 provides a descriptive analysis for the $\mathrm{CoVaR}$ and $\Delta$ CoVaR measures (in percentages) across debt markets. The results for the preonset period corroborate the graphical evidence: across debt markets, average $\mathrm{CoVaR}$ and ${ }_{\Delta} \mathrm{CoVaR}$ values were similar and standard deviations were of a similar magnitude. Descriptive statistics for the $\mathrm{CoVaR}$ values also indicate very similar results, irrespective of whether the CoVaR was computed through a bivariate

\footnotetext{
${ }^{6}$ Results at the $95 \%$ confidence level are available on request.

${ }^{7}$ The VaR values for any European debt market $\mathrm{j}$ at any time $\mathrm{t}$ is computed from the marginal models as $\operatorname{VaR}_{\alpha, \mathrm{t}}^{\mathrm{j}}=\mu_{\mathrm{t}}-\mathrm{t}_{\mathrm{v}, \eta}^{-1}(\alpha) \sqrt{\sigma_{\mathrm{j}, \mathrm{t}}^{2}}$, where $\mu_{\mathrm{t}}$ and $\sqrt{\sigma_{\mathrm{j}, \mathrm{t}}^{2}}$ are the conditional mean and standard deviation of the debt returns computed according to Eqs. (3.8)-(3.9) and where $t_{v, \eta}^{-1}(\alpha)$ denotes the $\alpha$-quantile of the skewed Student-t distribution in Eq. (3.10).
} 
Figure 3.4: $\mathrm{CoVaR}$ (left axis) and $\Delta \mathrm{CoVaR}$ (right axis) estimates for the European Economic and Monetary Union (EMU) with respect to selected countries.
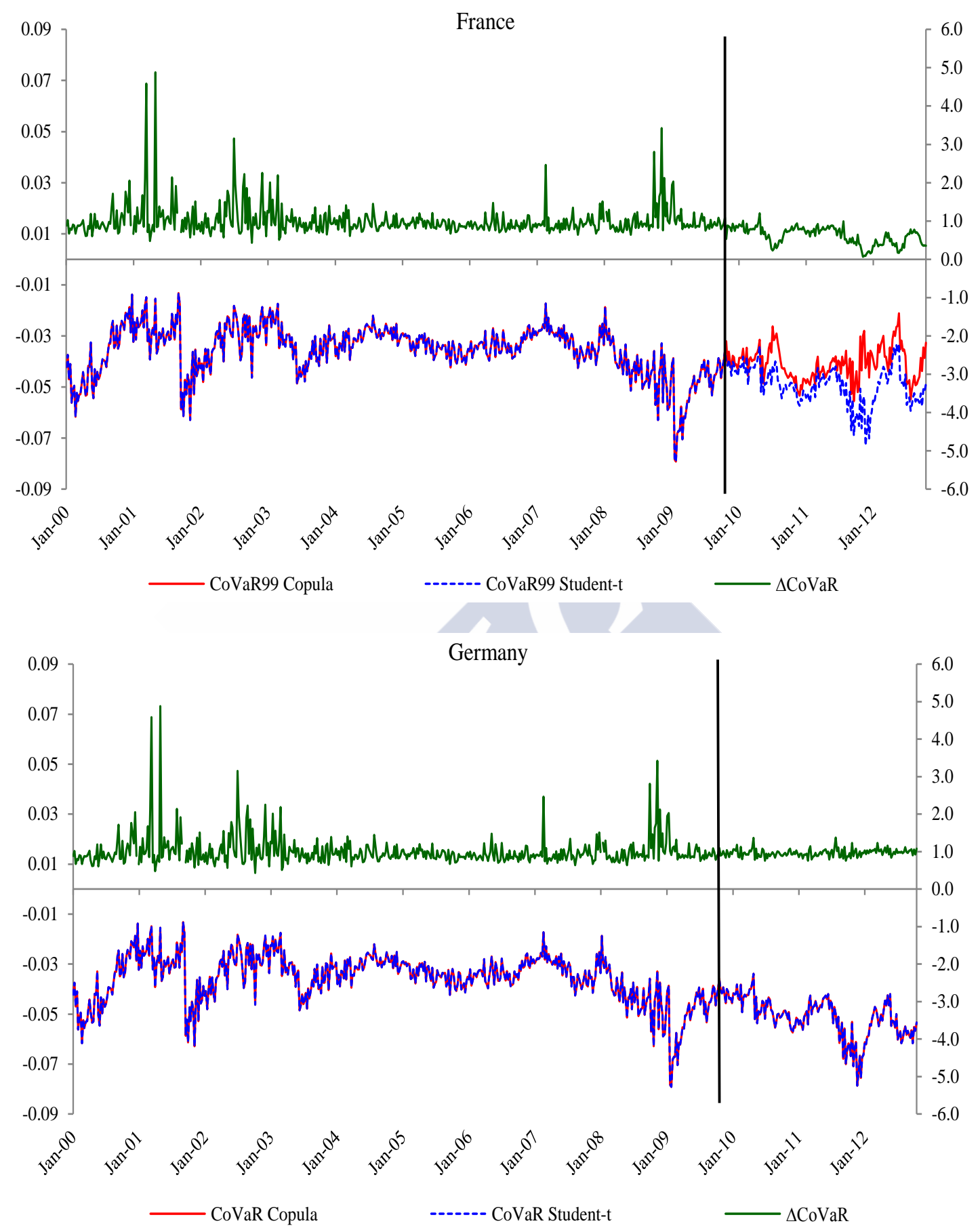
Figure 3.4: (Continued)
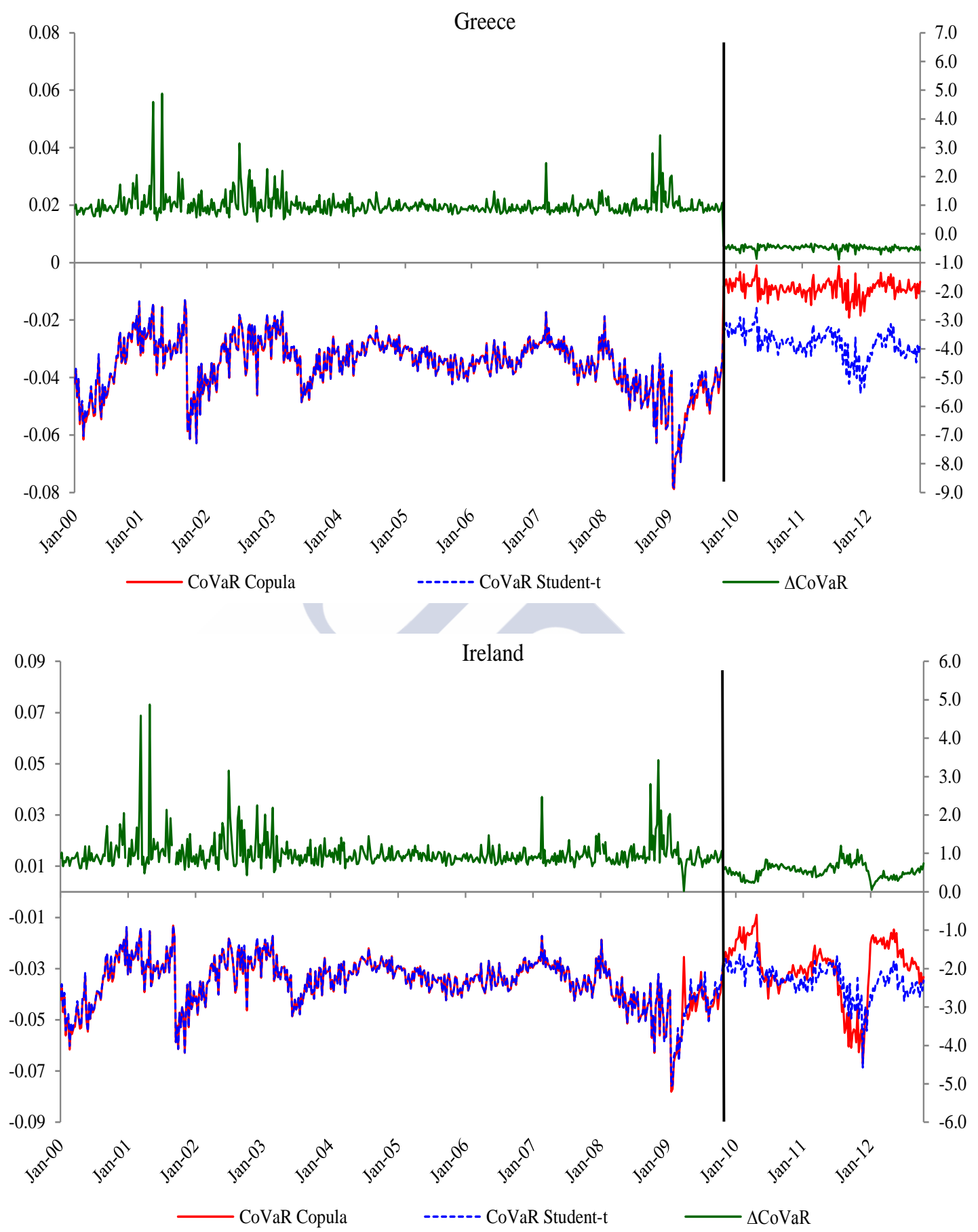
Figure 3.4: (Continued)
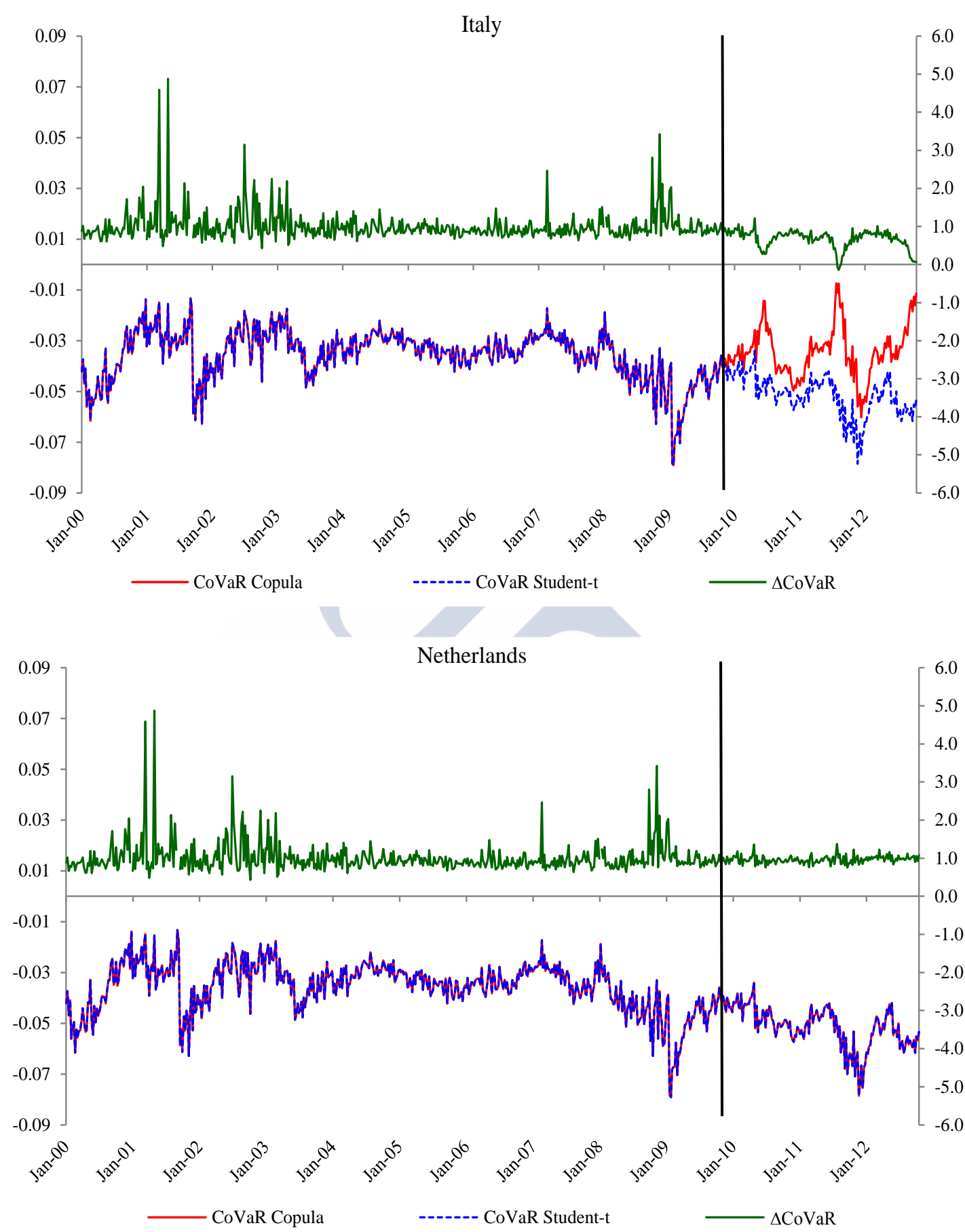
Figure 3.4: (Continued)
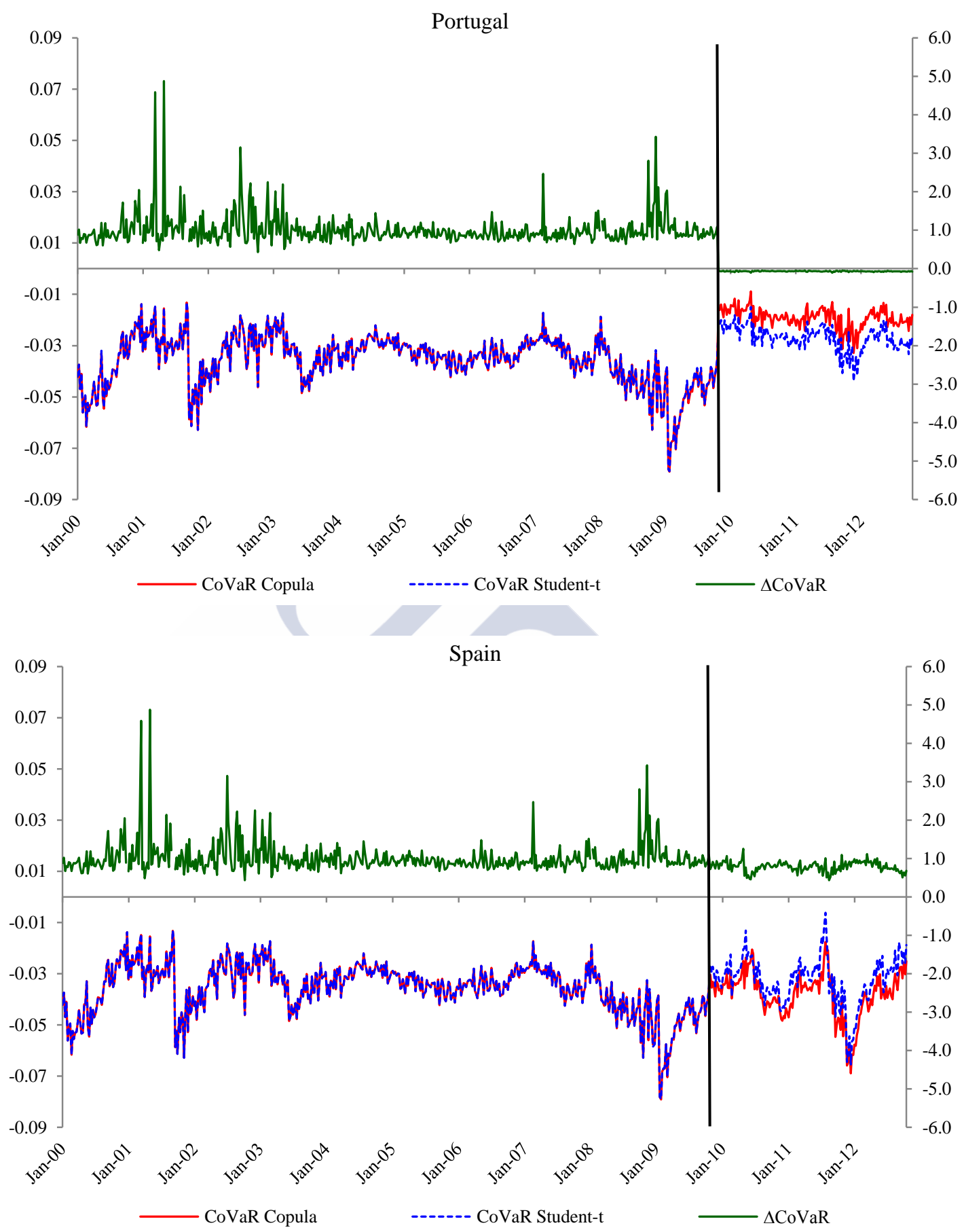
Student-t distribution or using a copula approach. Thus, regarding the pre-onset period, the copula approach to the CoVaR only had computational, not modelling, advantages, given that co-movement between debt markets and the EMU index was very high, with tail dependence also high for all copula specifications. In fact, there is no significant difference between the tail dependence arising from the TVProtated Gumbel copula (the best copula fit for almost all the markets) and the lower tail dependence arising from the Student-t copula. As a result, the CoVaR values for these two copula specifications did not differ too much, as Table 3.10 reports.

Regarding the post-onset period, the dynamics of the estimated CoVaR - as displayed in Figure 3.4 to the right of the vertical line and according to the descriptive statistics in Table 3.10-indicate that values increased for the GIIPS, with the exception of Spain. This is consistent with the fact that the debt markets of countries in crisis decoupled from other debt markets after the onset of the European sovereign debt market crisis. In fact, the dependence structure drastically changed in that the tail dependence of the pre-onset period dissipated after the onset of the debt crisis. The rise in the CoVaR values indicate that the systemic risk of the crisis countries was reduced, a finding corroborated by the behaviour of the $\triangle \mathrm{CoVaR}$ values, which, in some cases, dropped to negative values as a result of opposite movement between the country in question and the EMU index returns. In the case of Spain, lower tail dependence with the EMU index persisted, so CoVaR values dropped, thus furnishing evidence of an increase in systemic risk in the Spanish sovereign debt market. This result is consistent with the concerns of government authorities and financial media; it is also consistent with the fact that the Spanish debt market could be the drive-belt for the debt crisis in peripheral countries, bringing its repercussions to the hard core of Europe and generalizing the crisis to all European debt markets. In contrast, for the non-crisis countries, the CoVaR values dropped significantly, indicating that French, German and Dutch debt market risk became more systemic than in the pre-onset period. This result was a consequence of their decoupling from debt markets after the onset of the debt crisis; it was also due to the fact that the markets continued to strongly co-move with the EMU index. 
Table 3.10: Summary CoVaR and $\Delta \mathrm{CoVaR}$ statistics for the European Economic and Monetary Union (EMU) and selected countries.

\begin{tabular}{|c|c|c|c|c|c|c|c|c|c|}
\hline \multirow[b]{2}{*}{ Country } & \multirow[b]{2}{*}{ Method } & \multicolumn{4}{|c|}{ Before crisis onset } & \multicolumn{4}{|c|}{ After crisis onset } \\
\hline & & Mean & Std. & Max & Min & Mean & Std. & Max & Min \\
\hline \multirow{3}{*}{ France } & Student-t & -3.566 & 0.979 & -1.325 & -7.914 & -4.914 & 0.748 & -3.314 & -7.290 \\
\hline & Copula & -3.568 & 0.979 & -1.325 & -7.915 & -4.086 & 0.650 & -2.112 & -5.560 \\
\hline & $\Delta \mathrm{CoVaR}$ & 100.70 & 40.42 & 487.64 & 43.26 & 61.32 & 23.03 & 119.87 & 6.90 \\
\hline \multirow{3}{*}{ Germany } & Student-t & -3.569 & 0.979 & -1.325 & -7.915 & -5.131 & 0.806 & -3.396 & -7.856 \\
\hline & Copula & -3.569 & 0.979 & -1.325 & -7.914 & -5.131 & 0.806 & -3.396 & -7.856 \\
\hline & $\Delta \mathrm{CoVaR}$ & 100.71 & 40.42 & 487.68 & 43.26 & 96.77 & 10.39 & 137.42 & 75.64 \\
\hline \multirow{3}{*}{ Greece } & Student-t & -3.536 & 0.958 & -1.313 & -7.825 & -2.810 & 0.495 & -1.585 & -4.556 \\
\hline & Copula & -3.560 & 0.966 & -1.325 & -7.877 & -0.897 & 0.302 & -0.092 & -1.916 \\
\hline & $\Delta \mathrm{CoVaR}$ & 100.56 & 40.46 & 487.67 & 43.25 & -48.43 & 8.56 & -33.43 & -88.59 \\
\hline \multirow{3}{*}{ Ireland } & Student-t & -3.511 & 0.924 & -1.313 & -7.576 & -2.154 & 1.713 & 0.000 & -6.862 \\
\hline & Copula & -3.534 & 0.936 & -1.325 & -7.803 & -3.001 & 1.149 & -0.896 & -6.704 \\
\hline & $\Delta \mathrm{CoVaR}$ & 99.98 & 40.86 & 487.72 & 2.56 & 54.28 & 18.96 & 120.16 & 5.07 \\
\hline \multirow{3}{*}{ Italy } & Student-t & -3.566 & 0.979 & -1.324 & -7.913 & -5.129 & 0.806 & -3.394 & -7.853 \\
\hline & Copula & -3.568 & 0.978 & -1.325 & -7.904 & -3.490 & 1.063 & -0.231 & -6.337 \\
\hline & $\Delta \mathrm{CoVaR}$ & 100.69 & 40.42 & 487.66 & 43.25 & 66.13 & 26.43 & 118.17 & -47.64 \\
\hline \multirow{3}{*}{$\begin{array}{l}\text { Netherlan } \\
\text { ds }\end{array}$} & Student-t & -3.566 & 0.979 & -1.324 & -7.913 & -5.129 & 0.806 & -3.394 & -7.853 \\
\hline & Copula & -3.569 & 0.979 & -1.325 & -7.915 & -5.130 & 0.806 & -3.395 & -7.855 \\
\hline & $\Delta \mathrm{CoVaR}$ & 100.71 & 40.42 & 487.64 & 43.26 & 96.73 & 10.39 & 137.36 & 75.60 \\
\hline \multirow{3}{*}{ Portugal } & Student-t & -3.556 & 0.973 & -1.313 & -7.893 & -2.691 & 0.480 & -1.491 & -4.386 \\
\hline & Copula & -3.568 & 0.978 & -1.325 & -7.912 & -1.916 & 0.391 & -0.887 & -3.285 \\
\hline & $\Delta \mathrm{CoVaR}$ & 100.69 & 40.42 & 487.65 & 43.25 & -6.75 & 0.95 & -4.95 & -10.82 \\
\hline \multirow{3}{*}{ Spain } & Student-t & -3.562 & 0.977 & -1.320 & -7.893 & -3.253 & 0.942 & -0.622 & -6.540 \\
\hline & Copula & -3.568 & 0.977 & -1.325 & -7.911 & -3.793 & 0.896 & -1.760 & -6.890 \\
\hline & $\Delta \mathrm{CoVaR}$ & 100.70 & 40.42 & 487.65 & 43.26 & 78.65 & 13.56 & 125.20 & 43.60 \\
\hline
\end{tabular}

Notes. The table reports descriptive CoVaR and $\triangle \mathrm{CoVaR}$ statistics (percentages) at the $99 \%$ confidence level for the EMU and the VaR at the $99 \%$ level for selected countries in the pre- and postonset periods using the best copula fit and the multivariate generalized autoregressive conditional heteroskedasticity (MGARCH) model with bivariate Gaussian and Student-t distributions. Std., Max and Min denote standard deviation, maximum and minimum, respectively. 
Empirical evidence for the post-onset period also revealed differences for CoVaR measures computed using different methods. In general, for the non-crisis countries, dependence continued to be strong after the onset of the debt crisis, with lower tail dependence changing in some cases. Tail dependence for France was lower, explaining the differences between the copula approach and the bivariate Student-t approach that can be observed in Figure 3.4 and in Table 3.10. However, tail dependence for Germany remained high through different copula specifications, so there was no almost difference between the CoVaR values obtained through the BB1 copula or the Student-t approach. This evidence was similar for the Netherlands, with no significant differences.

Results for the countries in crisis in the post-onset period (Figure 3.4) provide striking evidence of differences in CoVaR values as computed using different approaches. These differences can be explained in terms of (lower) tail dependence: for the Greek and Portuguese markets there was tail independence, so the CoVaR values were reduced with respect to the values obtained using a bivariate Student-t distribution. Also, when there was (lower) tail dependence, as for the Italian, Portuguese and Spanish debt markets, CoVaR values computed using the copula approach differed with respect to the values for the bivariate Student-t distribution. Hence, one of the advantages of using copulas to compute the CoVaR lies in the fact that copulas offers more flexibility in terms of fitting dependence (and particularly tail dependence) than parametric bivariate distributions; consequently, they yield a more accurate $\mathrm{CoVaR}$ measure.

Overall, our results can be summarized as follows. Before the onset of the debt crisis, the dynamics and extent of systemic risk in European sovereign debt markets were similar, evidence consistent with high co-movement or coupling between debt markets. However, debt markets decoupled with the onset of the debt crisis, with crisis countries even displaying negative dependence. As a result, systemic risk decreased for the GIIPS debt markets, although not for Spain, and systemic risk increased for the non-crisis countries.

Our results have implications for investors in debt markets. First, evidence regarding decoupling after the onset of the crisis indicates that investors could find hedging opportunities using sovereign debt instruments; this would not have been possible before the crisis given the coupling between debt markets. Furthermore, our results regarding tail independence and reductions in systemic risk indicate that, after the onset of the debt crisis, investors could achieve downside risk reductions with a portfolio that included sovereign debt from different countries, mainly for 
sovereign debt markets that moved independently or in the opposite direction to the EMU index.

\subsubsection{Systemic risk in the Greek debt market}

Given that Greece was the leading protagonist of the European sovereign debt crisis, we estimated the $\mathrm{CoVaR}$ and $\triangle \mathrm{CoVaR}$ values for each European debt market conditional on the VaR of the Greek debt market in order to evaluate the systemic impact of the Greek crisis and its differential effects across debt markets before and after the onset of the crisis.

The dynamics of the CoVaR and ${ }_{\Delta} \mathrm{CoVaR}$ for all debt markets in the pre- and post-onset periods (delimited by a vertical line) are depicted in Figure 3.5. As with Figure 3.4, for each country we included information on the CoVaR computed using the methodology proposed by Girardi and Ergün (2013). The graphical evidence indicates that Greek systemic risk was low and relatively stable in the pre-onset period. The impact of the global financial crisis is reflected in an abrupt fall in the $\mathrm{CoVaR}$ value. Summary statistics for the $\mathrm{CoVaR}$ and $\Delta \mathrm{CoVaR}$ (in percentages) for the pre-onset period, as reported in Table 11, confirm that the systemic risk of the Greek debt market for other European debt markets was, on average, lower for the non-crisis countries than for the crisis countries.

However, Greek systemic risk drastically changed from the onset of the Greek debt crisis, with the $\mathrm{CoVaR}$ value associated with the crisis countries experiencing a huge reduction. In contrast, non-crisis countries experienced an increase in the $\mathrm{CoVaR}$ value given that they decoupled from the Greek market. Moreover, CoVaR volatility increased substantially for the countries in crisis as a result of the uncertainty of the debt markets and the implementation of stabilization policies as formulated by the European Central Bank and the International Monetary Fund, provoking sudden changes in investor expectations. This evidence on systemic risk dynamics is consistent with the idea that the crisis had spillover effects on countries with weak economic fundamentals, and also had contagion effects, given that countries like France, Germany and the Netherlands-with no great economic difficulties - reduced their conditional VaR. As for the crisis countries, Portugal experienced the greatest impact, with a fall in its CoVaR values of up to $-15 \%$ on average, followed by Ireland $(-13 \%)$, Spain $(-7.6 \%)$ and Italy $(-5.9 \%)$. This evidence suggests that the Greek debt crisis particularly affected Portugal. This is consistent with the concerns of the financial media regarding fears that the Greek crisis could rapidly extend to Portugal. 
Table 3.11: Summary CoVaR statistics for selected European debt markets and Greece.

\begin{tabular}{|c|c|c|c|c|c|c|c|c|c|}
\hline \multirow[b]{2}{*}{ Country } & \multirow[b]{2}{*}{ Method } & \multicolumn{4}{|c|}{ Before crisis onset } & \multicolumn{4}{|c|}{ After crisis onset } \\
\hline & & Mean & Std. & $\operatorname{Max}$ & Min & Mean & Std. & Max & Min \\
\hline \multirow{3}{*}{ France } & Student- $t$ & -3.980 & 1.080 & -1.680 & -10.249 & -3.658 & 1.312 & -1.573 & -8.207 \\
\hline & Copula & -4.023 & 1.119 & -1.688 & -10.274 & -1.876 & 0.716 & -0.244 & -4.275 \\
\hline & $\Delta \mathrm{CoVaR}$ & 114.24 & 43.10 & 550.10 & 4.81 & -7.96 & 2.38 & -5.54 & -32.22 \\
\hline \multirow{3}{*}{ Germany } & Student-t & -3.621 & 0.945 & -1.271 & -8.041 & -2.776 & 0.451 & -1.699 & -4.437 \\
\hline & Copula & -3.643 & 0.950 & -1.284 & -8.031 & -0.871 & 0.289 & 0.060 & -1.784 \\
\hline & $\Delta \mathrm{CoVaR}$ & 101.31 & 43.90 & 597.29 & 44.24 & -49.84 & 9.18 & -33.79 & -106.74 \\
\hline \multirow{3}{*}{ Ireland } & Student-t & -5.560 & 2.175 & -2.777 & -15.875 & -9.927 & 8.789 & 0.000 & -36.085 \\
\hline & Copula & -5.581 & 2.188 & -2.830 & -15.958 & -13.335 & 5.624 & -4.798 & -29.465 \\
\hline & $\Delta \mathrm{CoVaR}$ & 146.57 & 43.82 & 702.18 & 89.91 & 79.62 & 1.86 & 84.25 & 72.99 \\
\hline \multirow{3}{*}{ Italy } & Student-t & -4.402 & 1.786 & -1.641 & -14.113 & -7.399 & 3.086 & -3.624 & -15.844 \\
\hline & Copula & -4.252 & 1.724 & -0.563 & -13.585 & -5.962 & 2.502 & -3.027 & -12.845 \\
\hline & $\Delta \mathrm{CoVaR}$ & 111.88 & 28.45 & 321.86 & 22.50 & 59.53 & 2.30 & 66.84 & 47.97 \\
\hline \multirow{3}{*}{ Netherlands } & Student-t & -3.520 & 1.052 & -1.282 & -10.208 & -3.083 & 0.754 & -1.689 & -5.313 \\
\hline & Copula & -3.547 & 1.051 & -1.341 & -10.226 & -1.157 & 0.357 & -0.048 & -2.382 \\
\hline & $\Delta \mathrm{CoVaR}$ & 100.28 & 39.38 & 513.80 & 44.45 & -33.56 & 6.99 & -22.27 & -91.58 \\
\hline \multirow{3}{*}{ Portugal } & Student- $t$ & -5.330 & 2.321 & -2.399 & -18.431 & -23.683 & 8.277 & -6.366 & -42.892 \\
\hline & Copula & -5.335 & 2.324 & -2.405 & -18.432 & -15.167 & 4.916 & -3.932 & -28.369 \\
\hline & $\Delta \mathrm{CoVaR}$ & 135.06 & 27.30 & 355.42 & 82.11 & 44.68 & 21.55 & 95.68 & 1.14 \\
\hline \multirow{3}{*}{ Spain } & Student-t & -4.203 & 1.625 & -1.692 & -14.474 & -9.511 & 2.915 & -4.271 & -16.290 \\
\hline & Copula & -4.214 & 1.630 & -1.696 & -14.484 & -7.612 & 2.402 & -3.424 & -13.192 \\
\hline & $\Delta \mathrm{CoVaR}$ & 111.00 & 32.44 & 457.41 & 62.43 & 52.23 & 3.07 & 61.98 & 45.01 \\
\hline
\end{tabular}

Notes. The table reports descriptive CoVaR and $\triangle$ CoVaR statistics (percentages) at the $99 \%$ confidence level for the EMU and the VaR at the $99 \%$ level for Greece in the pre- and post-onset periods using the best copula fit and the multivariate generalized autoregressive conditional heteroskedasticity (MGARCH) model with bivariate Gaussian and Student-t distributions. Std., Max and Min denote standard deviation, maximum and minimum, respectively. 
Figure 3.5: CoVaR (left axis) and $\Delta \mathrm{CoVaR}$ (right axis) estimates for Greece with respect to selected countries.
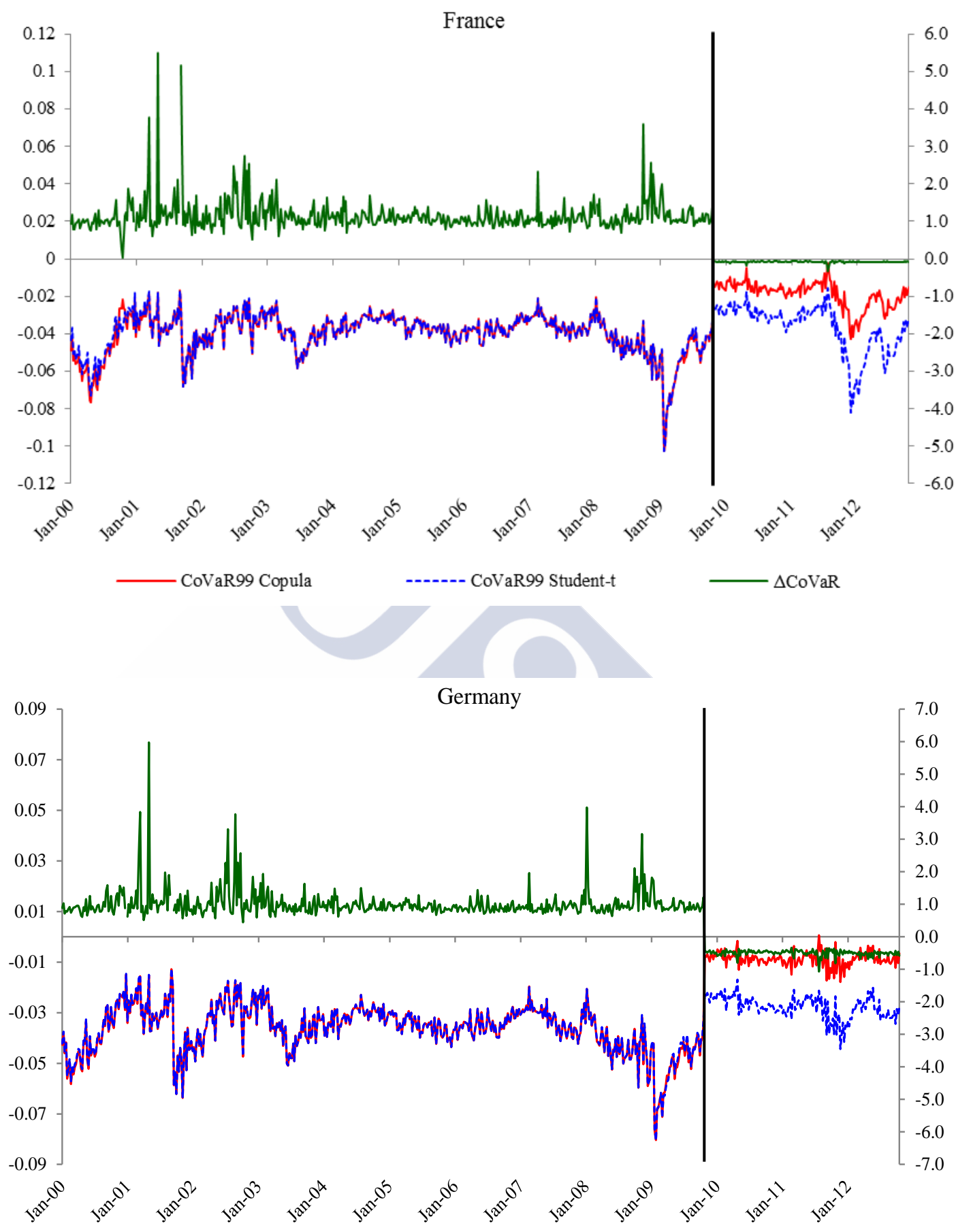
Figure: 3.5: (Continued)
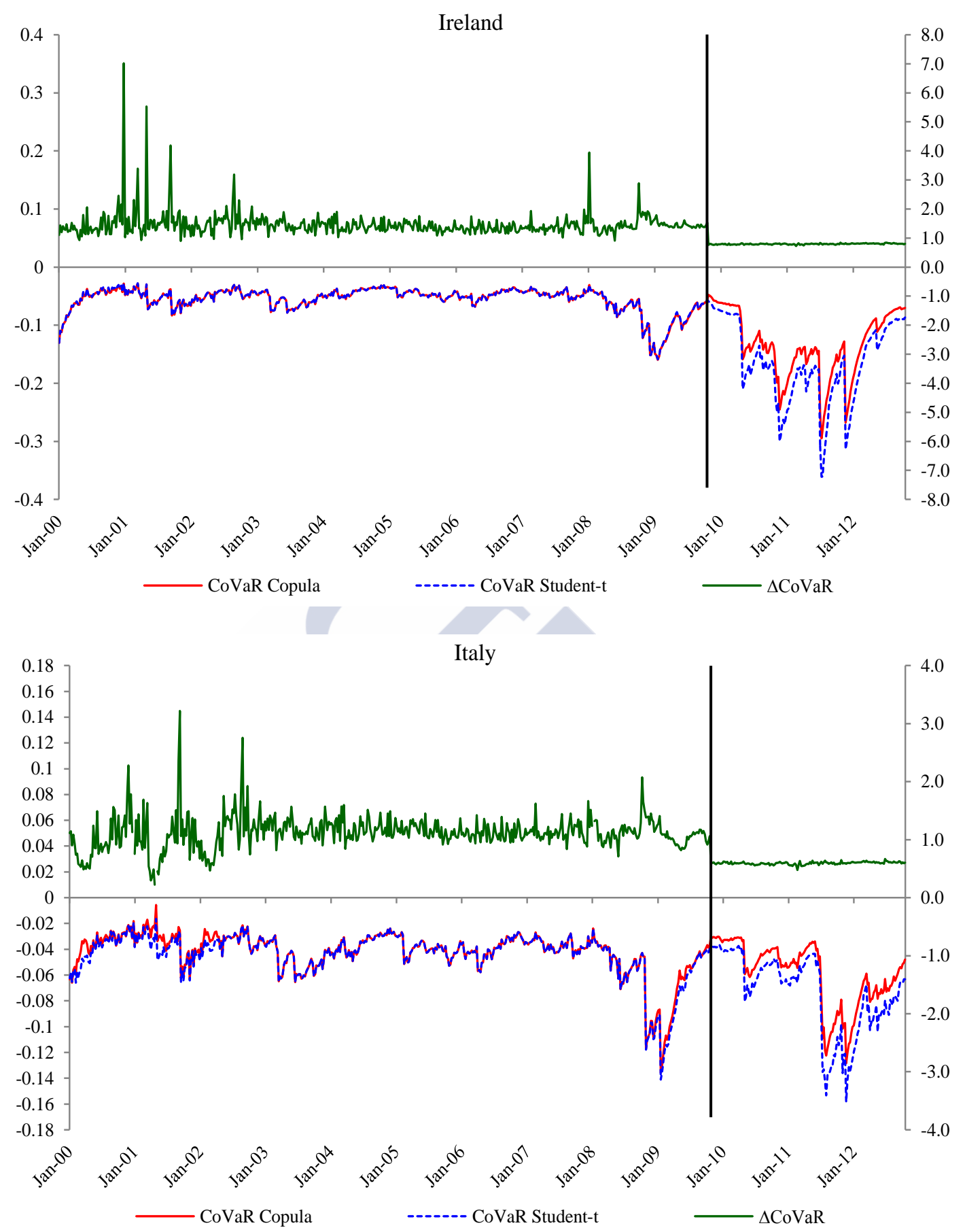
Figure: 3.5: (Continued)

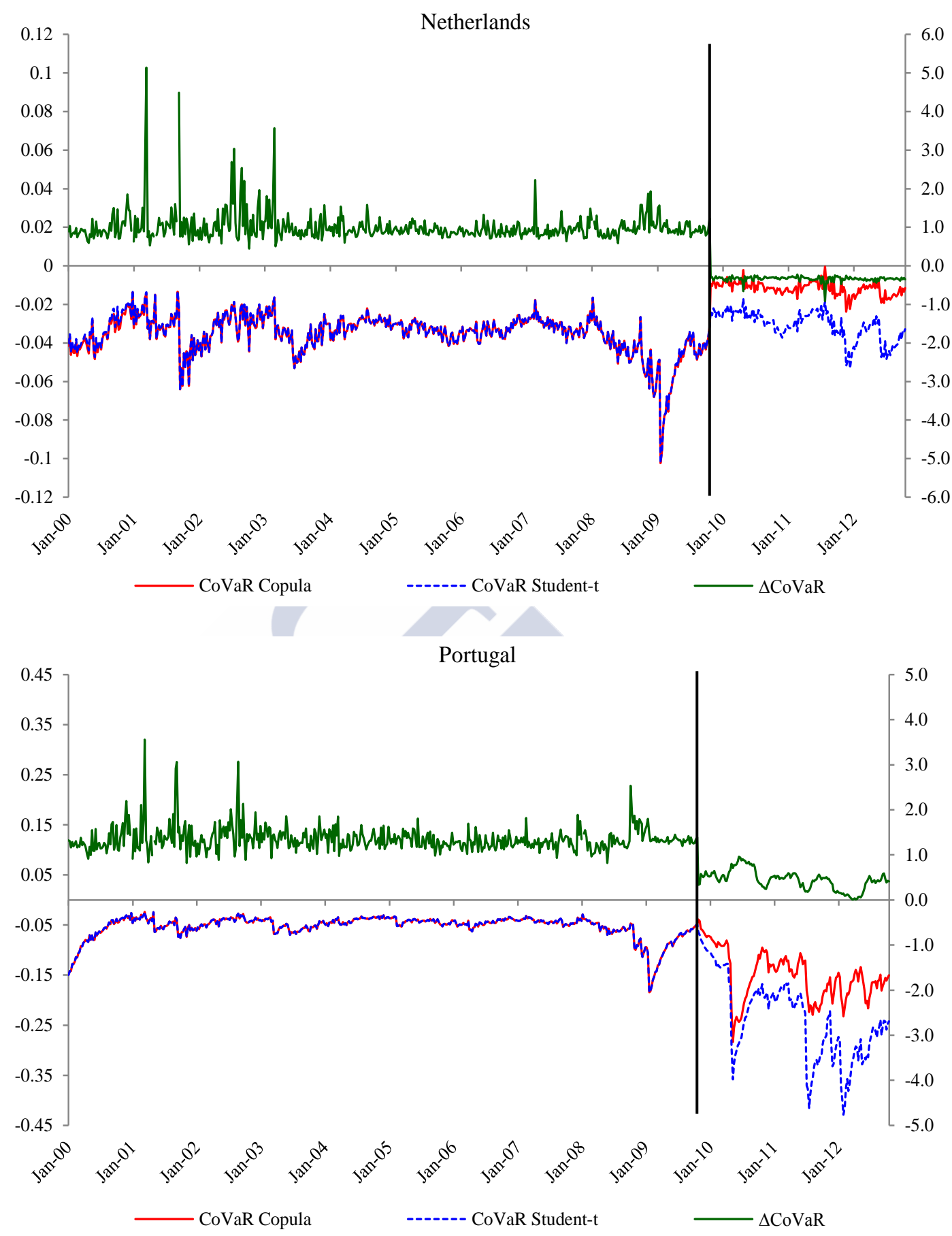


Figure: 3.5: (Continued)

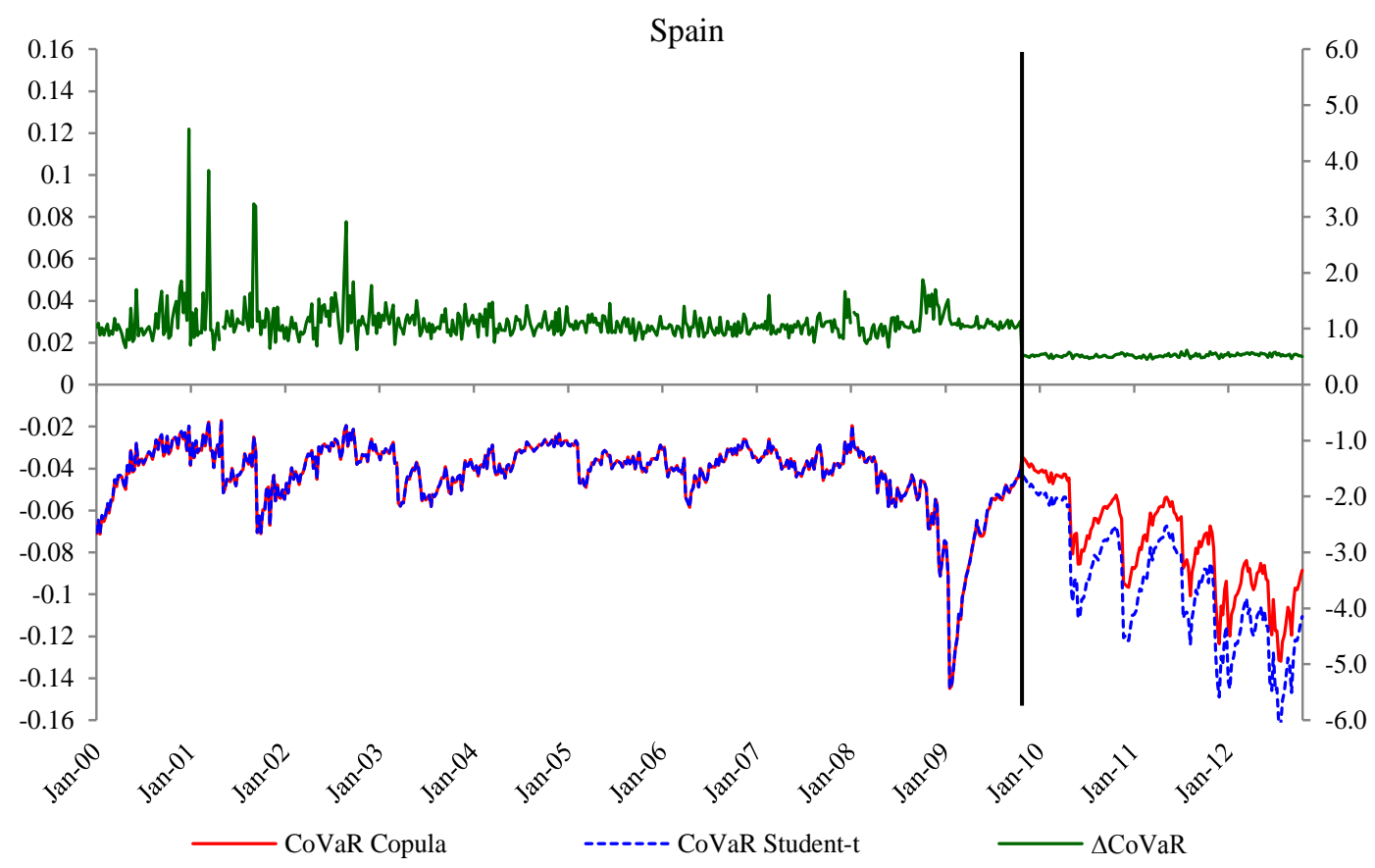

\subsection{Conclusions}

The recent European sovereign debt market crisis has raised investor and regulator concerns regarding the transmission of risk and contagion effects of a potential sovereign default in the euro area across debt markets. We measured systemic risk for European debt markets using the CoVaR measure, as proposed by Adrian and Brunnermeier (2011) and generalized by Girardi and Ergün (2013). The CoVaR is the $\mathrm{VaR}$ of a market conditional on the financial distress of another market, where financial distress is measured as the return of the distressed market taking values less or equal to its VaR. We computed the CoVaR using copulas given that the (lower) tail dependence of a copula function itself provides reliable information on the CoVaR. Copulas provide a flexible modelling dependence framework, since they provide information on both average and tail dependence; this information is crucial to determining the $\mathrm{CoVaR}$ value. Computing the $\mathrm{CoVaR}$ through copulas, which is not computationally cumbersome, involves a two-step procedure. First, from the copula function, the cumulative probability for the CoVaR is computed using information on the cumulative probability of the $\mathrm{VaR}$ of the market in financial distress and the confidence level for the CoVaR. Next, the marginal distribution function for this cumulative probability of the CoVaR is inverted in order to obtain the value of the CoVaR. Using a sample of sovereign bond benchmark price indices for France, Germany, the Netherlands and the GIIPS economies and an overall sovereign bond price index for the EMU for the period January 2000 to October 
2012, we calculated the systemic risk of the debt markets for each country and the systemic risk of the Greek debt market for other European debt markets for the periods before and after the onset of the European sovereign debt crisis. Our results indicate that European debt markets strongly co-moved in the period before the onset of the debt crisis and that systemic risk trends were similar across markets. However, after the onset of the crisis, European debt markets decoupled and GIIPS markets correlated negatively with the EMU index and displayed lower tail dependence. As a result, systemic risk changed dramatically and the value of the CoVaR increased. In contrast, for non-risk countries co-movement did not substantially change, even though systemic risk increased. We also analysed the systemic risk impact of Greek debt, finding that the risk for other European debt markets was low and stable before crisis onset. However, thereafter the systemic risk of Greek debt increased, mainly with regard to the other countries in crisis and with a particularly negative effect on the Portuguese market. The systemic risk of Greek debt for the non-crisis countries was reduced as a result of decoupling between the Greek debt market and the debt markets of the non-crisis countries. 


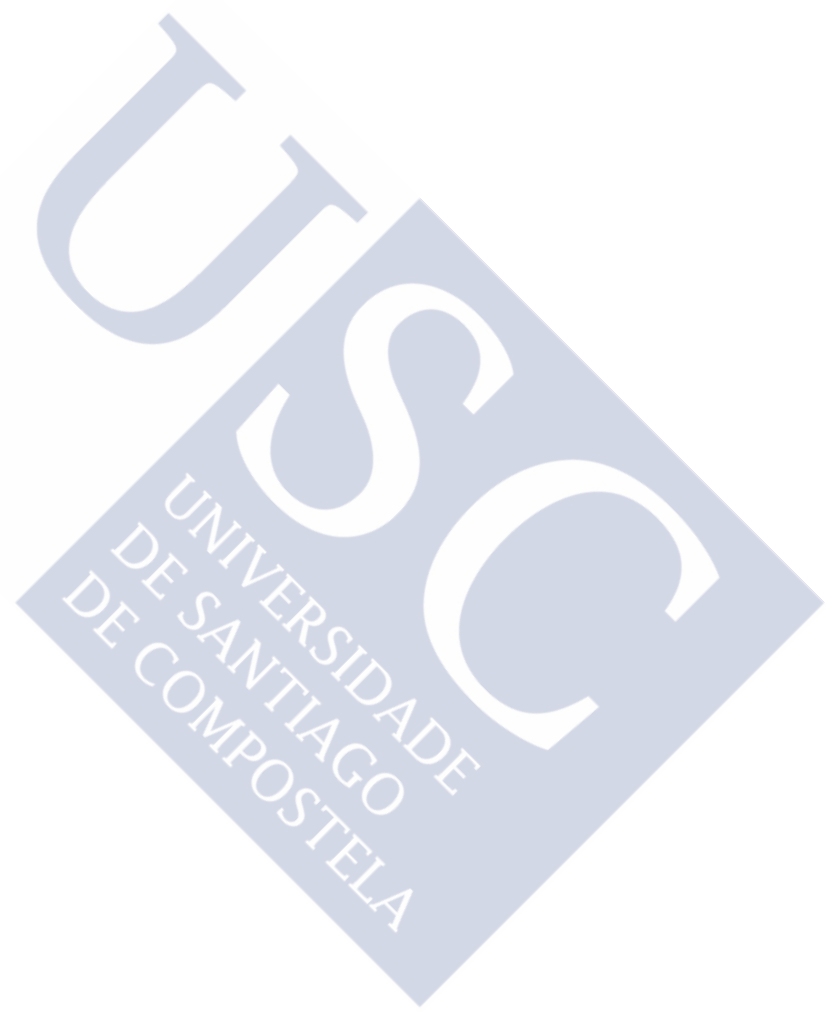




\section{Chapter 4}

\section{A Vine-copula CoVaR approach to systemic sovereign debt risk for the financial sector}

\subsection{Introduction}

How would a sovereign default impact on a country's financial system? Does sovereign default in one country impact on the financial systems of other countries? In view of the recent global financial and European debt crises, the answers to such questions have acquired great importance for investors, regulators and researchers.

We measured the systemic effect of domestic sovereign debt distress on domestic financial systems in European countries in the aftermath of recent financial and debt crises. We also measured the systemic effect of potential Greek debt distress on the financial systems of other European countries for the same period. Recent research has shown that systemic risk in financial markets increases considerably in times of crises and has adverse effects that extend to the real economy (e.g. Reinhart and Rogoff, 2009a). The links between financial crises and sovereign debt distress have been specifically documented by Reinhart and Rogoff (2009b, 2010) in relation to sovereign distress spreading to financial systems when banks hold substantial government debt in their portfolios (as happened in Italy, 
Portugal and, to a lesser extent, Spain).$^{8}$ Furthermore, sovereign debt portfolios of banks in European countries have been demonstrated to show a growing 'home bias', thereby increasing domestic sovereign holdings with sovereign solvency risk (Battistini, et al., 2014) while reinforcing the 'diabolic loop' between sovereign distress and bank solvency (Brunnermeier et al., 2011). Therefore, accurately quantifying how a potential sovereign default in the eurozone could impair the performance of a financial system has practical interest for both investors and regulators.

Over the last few years, researchers have developed an increasing number of systemic risk measures to account for the impact of failure of a financial institution on an entire financial system and vice versa. To capture possible risk spillovers between markets, Adrian and Brunnermeier (2011) proposed using conditional value-at-risk ( $\mathrm{CoVaR})$, a measure that provides information on the value-at-risk (VaR) of a market conditional on the fact that another market is in financial distress. This measure was later generalized by Girardi and Ergün (2013) by considering the VaR of a market conditional on the fact that another market's returns take values less than or equal to its VaR. Other authors have proposed alternative risk measures based on marginal expected shortfall (Acharya et al., 2010), extreme value theory (De Jonghe, 2010; Zhou, 2010), principal component analysis (Billio et al., 2012; Kritzman et al., 2011), default probabilities (Lehar, 2005; Huang et al., 2009; Segoviano and Goodhart, 2009; Huang et al., 2012; Gray and Jobst, 2010; Jin and Nadal De Simone, 2014), distance to default (Saldías, 2013) and network analysis (see, e.g., Halaj and Kok Sorensen, 2013; Allen et al., 2010; Tarashev et al., 2010). ${ }^{9}$

We chose CoVaR because VaR is arguably the risk measure most widely used by investors, financial institutions and regulators. We characterized CoVaR systemic risk in terms of copulas (Mainik and Schaanning, 2012). Firstly, to account for the effects of domestic sovereign debt risk on the domestic financial system, we employed bivariate copulas so that CoVaR could be obtained in a two-step procedure. Thus, for the confidence level for $\mathrm{CoVaR}$ and the cumulative probability of the $\mathrm{VaR}$ for the domestic sovereign debt market, we could compute CoVaR cumulative probability from a copula function. We then inverted the marginal

\footnotetext{
${ }^{8}$ Other channels of transmission of sovereign risk to financial institutions such as collateral, rating or guarantee channels were also identified by BIS (2011).

${ }^{9}$ Bisias et al. (2012) offer a comprehensive review on systemic risk measurement.
} 
distribution function for this cumulative probability in order to obtain CoVaR. Secondly, to account for the systemic impact of a potential Greek sovereign debt default on the financial system of other European countries, taking into account the link between domestic financial and sovereign debt markets we extended the copula $\mathrm{CoVaR}$ representation to a multivariate setting by considering the vine-copula approach, which allows systemic risk to be assessed using a hierarchical dependence construction given by a set of bivariate copulas. In this way, we could take into account dependence between the Greek sovereign market and the sovereign and financial systems of other countries; hence, the impact of sovereign default - in the domestic or Greek or both markets - on the financial system could be obtained in a three-step procedure: (1) for the given cumulative VaR probability and for the CoVaR confidence level, we computed the cumulative probability for the VaR referring to the domestic sovereign debt market from a copula function; (2) using the information obtained, we computed the cumulative CoVaR probability from a copula function; and finally, (3) we inverted the marginal distribution function for this cumulative probability in order to obtain CoVaR.

We measured the impact of sovereign debt default on the financial systems for both eurozone core countries (Austria, Belgium, Finland, France, Germany and the Netherlands) and peripheral countries (Italy, Greece, Portugal and Spain) for the period 2000 to 2012. Our results point to substantial differences in the systemic impact of sovereign debt on financial systems in the periods before and after crisis onset. Thus, before the outbreak of either the financial or debt crises (but mainly before the debt crisis), we observed that sovereign debt had a positive systemic risk impact in the sense that the financial system VaR increased; this increase can be explained in terms of the diversification effect of sovereign debt on bank portfolios. The Greek sovereign debt played a diversification role across financial systems in the entire eurozone excluding Portugal. However, with the onset of the European debt crisis domestic sovereign debt was observed to have a negative systemic impact on domestic financial systems. Not surprisingly, this effect was common to all the peripheral countries. As for the core countries, sovereign debt played a diversification role similar to that observed prior to the outbreak of the debt crisis. This differentiated sovereign debt impact can be explained in terms of the decoupling of debt markets across the eurozone; thus, negative effects on peripheral sovereign debt markets were not transmitted to core country sovereign debt markets. Furthermore, in examining the systemic impact of Greek sovereign debt distress on the financial systems of other countries, we found that although all 
European financial systems were affected, they were affected to different degrees. For core countries Greek sovereign debt continued to play a diversification role (even though the intensity was less and more varied); whereas for peripheral countries like Belgium and the Netherlands, Greek sovereign systemic effects exacerbated the risk associated with their financial systems.

This study adds to the burgeoning literature on the European sovereign debt crisis regarding links between sovereign debt markets and domestic and general financial sectors. De Bruyckere et al. (2013), for instance, examined contagion between bank and sovereign default risk in Europe through asset, collateral and rating channels. Bhanot et al. (2014) investigated how stock returns in the financial sector in crisis and non-crisis European countries were affected by the yield spreads for Greek sovereign debt. Mink and De Haan (2013) studied the impact of highly volatile Greek bonds on European bank stock prices in 2010. Alter and Schuler (2012) examined the relationship between sovereign default risk and domestic banking risk. For credit default swaps, other studies examined sovereign risk contagion among eurozone countries (e.g., Missio and Watzka, 2011; Arezki et al., 2011; Alter and Beyer, 2012; Caporin et al., 2013) and the effects of sovereign debt default risk on the financial stability of the eurozone (Radev, 2012). However, underrepresented in this literature is examination of the systemic impact of domestic sovereign distress on domestic financial systems or the impact of Greek sovereign debt distress on the financial systems of other countries. This chapter fills this gap by attempting to quantify $\mathrm{CoVaR}$ for financial and sovereign debt crises using procedures based on copulas and vine copulas.

The remainder of the chapter is laid out as follows: in Section 4.2 we outline the copula and vine-copula approaches to CoVaR, in Section 4.3 we present our data and in Section 4.4 we discuss the results. Finally, Section 4.5 concludes the chapter.

\subsection{Methodology}

We quantified the systemic impact of the sovereign debt market on the financial system using the CoVaR measure as introduced by Adrian and Brunnermeier (2011) and generalized by Girardi and Ergün (2013). CoVaR for the financial system of a country is $\mathrm{VaR}$ for the financial system conditional on the fact that the sovereign debt market is in financial distress. Let $\mathrm{x}_{\mathrm{t}}^{\mathrm{f}}$ be the returns for the financial system at time $t$ and let $x_{t}^{d}$ be the returns for the sovereign debt market at time t. Hence, 
CoVaR for a confidence level of $(1-\beta)$ can be formally characterized as the $\beta$ quantile of the conditional distribution of $x_{t}^{f}$ as follows:

$$
\operatorname{Pr}\left(\mathrm{x}_{\mathrm{t}}^{\mathrm{f}} \leq \operatorname{CoVaR}_{\beta, \mathrm{t}}^{\mathrm{f} \mid \mathrm{d}} \mid \mathrm{x}_{\mathrm{t}}^{\mathrm{d}} \leq \operatorname{VaR}_{\alpha, \mathrm{t}}^{\mathrm{d}}\right)=\beta,
$$

where $\operatorname{VaR}_{\alpha, t}^{\mathrm{d}}$ denotes the $\mathrm{VaR}$ of the debt market that measures the maximum loss that may be experienced by the sovereign debt market for a confidence level $1-\alpha$ at time t. Formally, it is the $\alpha$-quantile of the return distribution for the debt market: $\operatorname{Pr}\left(\mathrm{x}_{\mathrm{t}}^{\mathrm{d}} \leq \operatorname{VaR}_{\alpha, \mathrm{t}}^{\mathrm{d}}\right)=\alpha$.

We can compute CoVaR by determining the quantile of a conditional distribution or by using the quantile of an unconditional bivariate distribution, given that Eq. (4.1) can be written as:

$$
\frac{\operatorname{Pr}\left(\mathrm{x}_{\mathrm{t}}^{\mathrm{f}} \leq \operatorname{CoVaR}_{\beta, \mathrm{t}}^{\mathrm{f} \mid \mathrm{d}}, \mathrm{x}_{\mathrm{t}}^{\mathrm{d}} \leq \operatorname{VaR}_{\alpha, \mathrm{t}}^{\mathrm{d}}\right)}{\operatorname{Pr}\left(\mathrm{x}_{\mathrm{t}}^{\mathrm{d}} \leq \operatorname{VaR}_{\alpha, \mathrm{t}}^{\mathrm{d}}\right)}=\beta,
$$

or alternatively as:

$$
\operatorname{Pr}\left(\mathrm{x}_{\mathrm{t}}^{\mathrm{f}} \leq \operatorname{CoVaR}_{\beta, \mathrm{t}}^{\mathrm{f} \mid \mathrm{d}}, \mathrm{x}_{\mathrm{t}}^{\mathrm{d}} \leq \operatorname{VaR}_{\alpha, \mathrm{t}}^{\mathrm{d}}\right)=\alpha \beta
$$

\subsubsection{CoVaR with copulas}

To obtain CoVaR from Eq. (4.3), we used copulas to characterize the joint distribution function. ${ }^{10}$ Eq. (4.3) can be expressed in terms of the joint distribution function of $x_{t}^{f}$ and $x_{t}^{d}, F_{f, d}$, as $F_{f, d}\left(\operatorname{CoVaR}_{\beta, t}^{f \mid d}, \operatorname{VaR}_{\alpha, t}^{d}\right)=\alpha \beta$; furthermore, Sklar's (1959) theorem relates the joint distribution function and the copula as follows:

$$
F_{f, d}\left(x_{t}^{f}, x_{t}^{d}\right)=C\left(u_{f}, u_{d}\right)
$$

where $\mathrm{C}(\cdot, \cdot)$ is a copula function, $\mathrm{u}_{\mathrm{f}}=\mathrm{F}_{\mathrm{f}}\left(\mathrm{x}_{\mathrm{t}}^{\mathrm{f}}\right)$ and $\mathrm{u}_{\mathrm{d}}=\mathrm{F}_{\mathrm{d}}\left(\mathrm{x}_{\mathrm{t}}^{\mathrm{d}}\right)$ and where $\mathrm{F}_{\mathrm{f}}$ and $\mathrm{F}_{\mathrm{d}}$ are the marginal distribution functions of $x_{t}^{f}$ and $x_{t}^{d}$, respectively. Consequently, we can express Eq. (4.3) in terms of copulas as:

$$
\mathrm{C}\left(\mathrm{F}_{\mathrm{f}}\left(\operatorname{CoVaR}_{\beta, \mathrm{t}}^{\mathrm{f} \mid \mathrm{d}}\right), \mathrm{F}_{\mathrm{d}}\left(\operatorname{VaR}_{\alpha, \mathrm{t}}^{\mathrm{d}}\right)\right)=\alpha \beta .
$$

Hence, CoVaR can be computed from Eq. (5) using a simple two-step procedure:

\footnotetext{
${ }^{10}$ For further analysis on copulas, see Joe (1997) and Nelsen (2006). An overview of copula applications to finance can be found in Cherubini et al. (2004). Mainik and Schaanning (2012) provide the first representation of $\mathrm{CoVaR}$ in terms of bivariate copulas.
} 
(1) We obtain the value of $F_{f}\left(\operatorname{CoVaR}_{\beta, t}^{\mathrm{f} / \mathrm{d}}\right)$ from Eq. (4.5). Given that $C\left(\mathrm{u}_{\mathrm{f}}, \mathrm{u}_{\mathrm{d}}\right)=\alpha \beta$, where $\alpha, \beta$ and $u_{d}$ are given (note that $u_{d}=\alpha$ ), from the copula function specification we can solve to determine the value of $u_{f}=F_{f}\left(\operatorname{CoVaR}_{\beta, t}^{\mathrm{f}}\right)$.

(2) From $u$ we can obtain CoVaR as the quantile of the distribution of $x_{t}^{f}$, with a cumulative probability equal to $\mathrm{u}$, by inverting the marginal distribution function of $x_{t}^{f}: \operatorname{CoVaR}_{\beta, t}^{\mathrm{f} \mid \mathrm{d}}=\mathrm{F}_{\mathrm{f}}^{-1}\left(\mathrm{u}_{\mathrm{f}}\right)$.

The use of copulas to obtain $\mathrm{CoVaR}$ is appealing because of their flexibility, compared to parametric bivariate functions, in allowing separate modelling of the marginals and the dependence structure. This is crucial because marginals and dependence functions may have different tail dependence characteristics that may affect CoVaR. Furthermore, computing CoVaR using the above two-step procedure is simple and only requires information on the confidence levels. In fact, tail dependence information from copulas naturally provides a measure of CoVaR, even though it does so at the limits.

\subsubsection{CoVaR with vine copulas}

Copula CoVaR provides useful information in a bivariate setup. However, since we wanted to consider systemic risk affecting several markets-i.e., the impact of Greek debt distress on the financial system and on other debt markets - we needed to consider dependence in more than two dimensions. We thus considered vine copulas, ${ }^{11}$ since these account for a multivariate distribution that combines three or more marginal distributions in a joint distribution. Vine copulas are multivariate copulas that are generated through a hierarchical construction that is decomposed into a cascade of bivariate copulas called pair-copulas, where each bivariate paircopula captures conditional dependence between two variables. Thus, a vine construction requires pairs of original variables and pairs of conditional distributions of recomputed variables to be modelled.

Since we wished to analyse the systemic impact of a distressed sovereign debt market (Greece) and foreign debt markets on national financial systems, we

\footnotetext{
${ }^{11}$ In the statistical literature vine copulas were introduced by Joe (1997) and were extended by Aas et al. (2009) for risk management purposes. Some applications of vine copulas in finance include, among others, Chollete et al. (2009), Aas and Beng (2009), Low et al. (2013) and Weiß and Supper (2013).
} 
considered a vine copula with three variables. Let $\mathrm{x}_{\mathrm{t}}^{\mathrm{d}^{*}}$ be the returns for the foreign debt market at time $t$ with distribution function $\mathrm{F}_{\mathrm{d}^{*}}\left(\mathrm{x}_{\mathrm{t}}^{\mathrm{d}^{*}}\right)=\mathrm{u}_{\mathrm{d}^{*}}$. According to Bedford and Cooke (2001), the joint density of financial, national and foreign debt returns can be expressed as the product of the marginal densities and a set of conditional bivariate copulas as:

$$
\begin{aligned}
f\left(x^{f}, x^{d}, x^{d^{*}}\right)= & c_{f, d \mid d^{*}}\left(F_{f \mid d^{*}}\left(x^{f} \mid x^{d^{*}}\right), F_{d \mid d^{*}}\left(x^{d} \mid x^{d^{*}}\right)\right) c_{f, d^{*}}\left(F_{f}\left(x^{f}\right), F_{d^{*}}\left(x^{d^{*}}\right)\right) \\
& c_{d, d^{*}}\left(F_{d}\left(x^{d}\right), F_{d^{*}}\left(x^{d^{*}}\right)\right) f_{f}\left(x^{f}\right) f_{d}\left(x^{d}\right) f_{d^{*}}\left(x^{d^{*}}\right)
\end{aligned}
$$

where $c(\cdot, \cdot)$ denotes the copula density and $f(\cdot)$ the marginal densities and where $\mathrm{c}_{\mathrm{f}, \mathrm{d} \mid \mathbf{d}^{*}}$ is referred to as the pair-copula. The conditional distribution functions in Eq. (4.6) for any two random variables $\mathrm{x}$ and $\mathrm{y}$ can be obtained as (Joe, 1997):

$$
\mathrm{F}(\mathrm{x} \mid \mathrm{y})=\frac{\partial \mathrm{C}_{\mathrm{x}, \mathrm{y}}(\mathrm{F}(\mathrm{x}), \mathrm{F}(\mathrm{y}))}{\partial \mathrm{F}(\mathrm{y})} .
$$

The decomposition in Eq. (4.6) is a canonical or C-vine copula model where the initial node of the vine copula hierarchical structure is given by the returns of the foreign debt market; alternatively, the decomposition in Eq. (4.6) is given by a Dvine copula model like that represented in Figure 4.1, where each edge corresponds to a bivariate copula density and the first three $T_{1}$ (upper) nodes correspond to the marginals. We adopted this hierarchical structure as we were interested in the systemic impact of the Greek national debt market on the financial system, given specific foreign debt market circumstances, or the systemic effect of sovereign Greek debt distress on the financial systems of other countries.

Figure 4.1: A D-Vine copula hierarchical structure

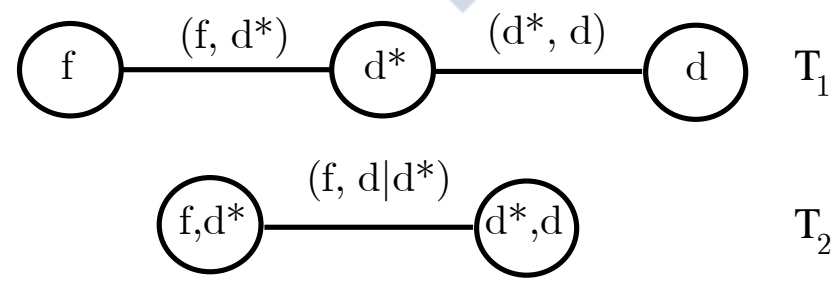

Now, in this multivariate conditional setup, CoVaR is given by:

$$
\operatorname{Pr}\left(\mathrm{x}_{\mathrm{t}}^{\mathrm{f}} \leq \operatorname{CoVaR}_{\beta, \mathrm{t}}^{\mathrm{f} \mid \mathrm{d} \mathrm{d}^{*}} \mid \mathrm{x}_{\mathrm{t}}^{\mathrm{d}} \leq \operatorname{VaR}_{\alpha, \mathrm{t}}^{\mathrm{d} \mid \mathrm{d}^{*}}, \mathrm{x}_{\mathrm{t}}^{\mathrm{d}^{*}}\right)=\beta
$$

which can be expressed in terms of the conditional joint distribution function as $\mathrm{F}_{\mathrm{f}, \mathrm{d} \mid \mathrm{d}^{*}}\left(\operatorname{CoVaR}_{\beta, \mathrm{t}}^{\mathrm{f} \mid \mathrm{d} \mathrm{d}^{*}}, \operatorname{VaR}_{\alpha, \mathrm{t}}^{\mathrm{d} \mid \mathrm{d}^{*}}\right)=\alpha \beta$. Hence, to obtain CoVaR from the vine-copula 
specification, we have to take into account information provided by the conditional joint distribution function of $x_{t}^{f}$ and $x_{t}^{d}$, given, in terms of the copula, as:

$$
F_{f, d \mid d^{*}}\left(\left(x^{f} \mid x^{d^{*}}\right),\left(x^{d} \mid x^{d^{*}}\right)\right)=C_{f, d \mid d^{*}}\left(\left(u_{f} \mid u_{d^{*}}\right),\left(u_{d} \mid u_{d^{*}}\right)\right) .
$$

Thus, CoVaR can be obtained from the vine copula using a three-steep procedure:

(1) For given values of $\beta, u_{d}=\alpha$ and $u_{d^{*}}$ and for the copula specification in Eq. (4.9) we can solve to determine the value of $\left(\mathrm{u}_{\mathrm{f}} \mid \mathrm{u}_{\mathrm{d}^{*}}\right)$.

(2) From the value of $\left(u_{f} \mid u_{d^{*}}\right)$ we obtain the value of $u_{f}$ by solving from the conditional distribution of $\mathrm{x}_{\mathrm{t}}^{\mathrm{f}}$ given by Eq. (4.7).

(3) From $u_{f}$ we obtain CoVaR as the quantile of the distribution of $x_{t}^{f}$, with a cumulative probability equal to $\mathrm{u}$, by inverting the marginal distribution function of $x_{t}^{f}: \operatorname{CoVaR}_{\beta, t}^{f \mid d, d^{*}}=F_{f}^{-1}\left(u_{f}\right)$.

Following this three-step procedure we obtain information on CoVaR of the financial system in a given country in a situation of debt market distress, taking into account the foreign debt market situation. Furthermore, we can also consider the CoVaR of the financial system under two other market scenarios: (1) both national and foreign debt markets are distressed $\left(\mathrm{x}_{\mathrm{t}}^{\mathrm{d}} \leq \operatorname{VaR}_{\alpha, \mathrm{t}}^{\mathrm{d}}\right.$ and $\left.\mathrm{x}_{\mathrm{t}}^{\mathrm{d}^{*}} \leq \operatorname{VaR}_{\gamma, \mathrm{t}}^{\mathrm{d}^{*}}\right) ;(2)$ only

the foreign debt market is distressed $\left(\mathrm{x}_{\mathrm{t}}^{\mathrm{d}^{*}} \leq \mathrm{VaR}_{\gamma, \mathrm{t}}^{\mathrm{d}^{*}}\right)$. In the first case, the returns in both debt markets are below or equal to their VaR figures, so $u_{d}=\alpha$ and $u_{d^{*}}=\gamma$, where $1-\gamma$ is the confidence level for the $\operatorname{VaR}$ of the foreign debt market. The estimation procedure is identical to the three-steep procedure described above except regarding the value of $u_{d^{*}}$. In the second case, we only have information on $u_{d^{*}}=\gamma$ and for that information we have to obtain the value of $u_{d}$ from the bivariate copula for both debt markets. Once we have this information we follow the threestep procedure described above.

\subsubsection{Marginal distribution and copula models}

The marginal models and copula specifications used to compute the CoVaR measures are described as follows.

To account for the usual characteristics of financial return distributions, such as leverage, fat tails and asymmetries, we considered that the conditional mean and 
variance of returns $\left(r_{t}\right)$ are given by an autoregressive moving average (ARMA) model with $\mathrm{p}$ and $\mathrm{q}$ lags specified as:

$$
\mathrm{r}_{\mathrm{t}}=\phi_{0}+\sum_{\mathrm{j}=1}^{\mathrm{p}} \phi_{\mathrm{j}} \mathrm{r}_{\mathrm{t}-\mathrm{j}}+\sum_{\mathrm{h}=1}^{\mathrm{q}} \varphi_{\mathrm{j}} \varepsilon_{\mathrm{t}-\mathrm{h}}+\varepsilon_{\mathrm{t}}
$$

where $\varepsilon_{\mathrm{t}}=\sigma_{\mathrm{t}} \mathrm{z}_{\mathrm{t}}$ and where $\sigma_{\mathrm{t}}^{2}$ is the conditional variance, given by a threshold generalized autoregressive conditional heteroskedasticity (TGARCH) specification (Zakoian, 1994; Glosten et al., 1993):

$$
\sigma_{\mathrm{t}}^{2}=\omega+\sum_{\mathrm{k}=1}^{\mathrm{r}} \mathrm{b}_{\mathrm{k}} \sigma_{\mathrm{t}-\mathrm{k}}^{2}+\sum_{\mathrm{h}=1}^{\mathrm{m}} \mathrm{a}_{\mathrm{h}} \varepsilon_{\mathrm{t}-\mathrm{h}}^{2}+\sum_{\mathrm{h}=1}^{\mathrm{m}} \lambda_{\mathrm{h}} \varepsilon_{\mathrm{t}-\mathrm{h}}
$$

where $\omega$ is a constant, $\sigma_{\mathrm{t}-\mathrm{k}}^{2}$ is the GARCH component and $\varepsilon_{\mathrm{t}-\mathrm{h}}$ is the ARCH component. The parameter $\lambda$ captures asymmetric effects in such a way that when $\lambda<0$, the future conditional variance will proportionally increase more following a negative shock than following a positive shock of the same magnitude. $z_{t}$ is a zero mean and unit variance i.i.d. random variable that follows a Hansen's (1994) skewed-t density distribution given by:

$$
f\left(z_{t} ; v, \eta\right)=\left\{\begin{array}{l}
b c\left(1+\frac{1}{v-2}\left(\frac{b z_{t}+a}{1-\eta}\right)^{2}\right)^{-(v+1) / 2} \quad z_{t}<-a / b \\
b c\left(1+\frac{1}{v-2}\left(\frac{b z_{t}+a}{1+\eta}\right)^{2}\right)^{-(v+1) / 2} \\
z_{t} \geq-a / b
\end{array},\right.
$$

where $v$ and $\eta$ are the degrees of freedom parameter $(2<v<\infty)$ and the symmetric parameter $(-1<\eta<1)$, respectively. The constants $\mathrm{a}, \mathrm{b}$ and $\mathrm{c}$ are given by $\mathrm{a}=4 \eta \mathrm{c}\left(\frac{\mathrm{v}-2}{\mathrm{v}-1}\right), \mathrm{b}^{2}=1+3 \eta^{2}-\mathrm{a}^{2}$ and $\mathrm{c}=\Gamma\left(\frac{\mathrm{v}+1}{2}\right) / \sqrt{\pi(\mathrm{v}-2)} \Gamma\left(\frac{\mathrm{v}}{2}\right)$. This distribution converges to the standard Gaussian as $\eta=0$ and as $v \rightarrow \infty$, and to the symmetric Student-t distribution as $\eta=0$ and $v$ is finite.

We used seven different copula specifications to capture different characteristics of dependence: tail independence (Gaussian and Plackett), symmetric tail dependence (Student-t) and asymmetric tail dependence (Gumbel, Rotated Gumbel, BB7 and Symmetric Joe-Clayton (SJC)). Table 4.1 summarizes the main features of all the static and dynamic copula functions that were employed in the empirical analysis.

We estimated the parameters of the marginal and bivariate copula models following the inference function for margins procedure (Joe and $\mathrm{Xu}, 1996$ ), which consists of first estimating the parameters of the marginal distributions separately 
using maximum likelihood and then estimating the parameters of the copula using the pseudo-sample observations for the copula given by the probability integral transformation of the standardized residuals for the marginals. For the vine copula (second tree of Figure 4.1), we recomputed the pseudo-sample observations through the copulas estimated for the first tree. This sequential estimation procedure was introduced by Aas et al. (2009) and later examined in Hobæk Haff (2013). The number of lags in the mean and variance equations for each series was selected according to the Akaike information criteria (AIC) and the different copula models were evaluated using the AIC adjusted for small-sample bias, as in Breymann et al. (2003) and Reboredo (2011; 2013).

\subsection{Data}

We empirically examined the systemic risk effect of sovereign debt distress on the financial sector by considering six eurozone core countries (Austria (AT), Belgium (BE), Finland (FI), France (FR), Germany (DE) and Netherlands (NL)) and four peripheral countries (Italy (IT), Greece (GR), Portugal (PT) and Spain (ES)). For each country we considered weekly data for benchmark bond price indices for 10year maturities and for the MSCI financial price index. Data were sourced from Datastream and Bloomberg and cover the period 23 December 1999 to 25 May 2012. With this data we evaluated the following: (1) the impact of a distress event in one country's debt market on its financial sector as represented by the MSCI financial index; (2) the impact of a distress event in the Greek debt market on the banking sector of other European countries; and (3) the impact of a simultaneous distress event in the Greek and domestic debt markets on the domestic financial system. 
A Vine-copula CoVaR approach to systemic sovereign debt risk for the financial...

Table 4.1: Copula models.

\begin{tabular}{|c|c|c|c|}
\hline Name & Copula & Parameter & Structure dependence \\
\hline Gaussian & $\mathrm{C}_{\mathrm{N}}(\mathrm{u}, \mathrm{v} ; \rho)=\Phi\left(\Phi^{-1}(\mathrm{u}), \Phi^{-1}(\mathrm{v})\right)$ & $\rho$ & No tail dependence. $\tau^{\mathrm{U}}=\tau^{\mathrm{L}}=0$ \\
\hline Student-t & $\mathrm{C}_{\mathrm{ST}}(\mathrm{u}, \mathrm{v} ; \rho, \mathrm{v})=\mathrm{T}\left(\mathrm{t}_{\mathrm{v}}^{-1}(\mathrm{u}), \mathrm{t}_{\mathrm{v}}^{-1}(\mathrm{v})\right)$ & $\rho, v$ & $\begin{array}{l}\text { Symmetric tail dependence: } \\
\lambda_{\mathrm{U}}=\lambda_{\mathrm{L}}=2 \mathrm{t}_{\mathrm{v}+1}(-\sqrt{\mathrm{v}+1} \sqrt{1-\rho} / \sqrt{1+\rho})\end{array}$ \\
\hline Gumbel & $\mathrm{C}_{\mathrm{G}}(\mathrm{u}, \mathrm{v} ; \delta)=\exp \left(-\left((-\log \mathrm{u})^{\delta}+(-\log \mathrm{v})^{\delta}\right)^{1 / \delta}\right)$ & $\delta \geq 1$ & $\begin{array}{l}\text { Asymmetric tail dependence: } \\
\lambda_{\mathrm{L}}=0, \lambda_{\mathrm{U}}=2-21 / \theta\end{array}$ \\
\hline Rotated Gumbel & $\mathrm{C}_{\mathrm{RG}}(\mathrm{u}, \mathrm{v} ; \delta)=\mathrm{u}+\mathrm{v}-1+\mathrm{C}_{\mathrm{G}}(1-\mathrm{u}, 1-\mathrm{v} ; \delta)$ & $\delta \geq 1$ & $\begin{array}{l}\text { Asymmetric tail dependence: } \\
\lambda_{\mathrm{L}}=0, \lambda_{\mathrm{U}}=2-2 \frac{1 / \theta}{}\end{array}$ \\
\hline BB7 & $\mathrm{C}_{\mathrm{BB} 7}(\mathrm{u}, \mathrm{v} ; \delta, \theta)=1-\left(1-\left[\left(1-(1-\mathrm{u})^{\theta}\right)^{-\delta}+\left(1-(1-\mathrm{v})^{\theta}\right)^{-\delta}-1\right]^{-1 / \delta}\right)^{1 / \theta}$ & $\begin{array}{l}\theta \geq 1 \\
\delta>0\end{array}$ & $\begin{array}{l}\text { Asymmetric tail dependence: } \\
\lambda_{\mathrm{L}}=2^{-1 / \delta}, \lambda_{\mathrm{U}}=2-21 / \theta\end{array}$ \\
\hline Plackett & $\mathrm{C}_{\mathrm{P}}(\mathrm{u}, \mathrm{v} ; \theta)=\frac{1}{2(\theta-1)}(1+(\theta-1)(\mathrm{u}+\mathrm{v}))-\sqrt{(1+(\theta-1)(\mathrm{u}+\mathrm{v}))^{2}-4 \theta(\theta-1) \mathrm{uv}}$ & $\begin{array}{l}\theta>0 \\
\theta \neq 1\end{array}$ & No tail dependence. $\tau^{\mathrm{U}}=\tau^{\mathrm{L}}=0$ \\
\hline SJC & $\begin{array}{l}\mathrm{C}_{\mathrm{SJC}}(\mathrm{u}, \mathrm{v} ; \tau \mathrm{U}, \tau \mathrm{L})=0.5 \cdot\left(\mathrm{C}_{\mathrm{JC}}\left(\mathrm{u}, \mathrm{v} ; \tau^{\mathrm{U}}, \tau \mathrm{L}\right)+\mathrm{C}_{\mathrm{JC}}\left(1-\mathrm{u}, 1-\mathrm{v} ; \tau^{\mathrm{U}}, \tau^{\mathrm{L}}\right)+\mathrm{u}+\mathrm{v}-1\right) \\
\text { where } \kappa=1 / \log _{2}(2-\tau \mathrm{U}), \gamma=-1 / \log _{2}(\tau \mathrm{L}), \mathrm{C}_{\mathrm{JC}}(\square) \text { is similar to } \mathrm{C}_{\mathrm{BB} 7}(\square)\end{array}$ & $\begin{array}{l}\tau^{\mathrm{U}} \in(0,1) \\
\tau^{\mathrm{L}} \in(0,1)\end{array}$ & $\begin{array}{l}\text { Upper and lower tail } \\
\text { independence: } \tau^{\mathrm{U}}=0 \text { and } \tau^{\mathrm{L}}=0\end{array}$ \\
\hline
\end{tabular}

Notes. We also captured time-varying dependence by assuming that copula parameters change over time. For the Gaussian and Student-t copulas, we assumed an $\operatorname{ARMA}(1, \mathrm{q})$-type process (Patton, 2006) for the linear dependence parameter $\rho_{\mathrm{t}}: \rho_{\mathrm{t}}=\Lambda_{1}\left(\psi_{0}+\psi_{1} \rho_{\mathrm{t}-1}+\psi_{2} \frac{1}{\mathrm{q}} \sum_{\mathrm{j}=1}^{\mathrm{q}} \Phi^{-1}\left(\mathrm{u}_{\mathrm{t}-\mathrm{i}}\right) \cdot \Phi^{-1}\left(\mathrm{v}_{\mathrm{t}-\mathrm{i}}\right)\right)$, where $\Lambda_{1}(\mathrm{x})=\left(1-\mathrm{e}^{-\mathrm{x}}\right)\left(1+\mathrm{e}^{-\mathrm{x}}\right)^{-1}$ is the modified logistic transformation that keeps the value of $\rho_{t}$ in $(-1,1)$. For the Student-t copula, $\Phi^{-1}(\mathrm{x})$ is replaced by $t_{v}^{-1}(\mathrm{x})$. We considered time-varying dependence for the Gumbel and Rotated Gumbel copulas by assuming that the parameters reflect the dynamics given by the following equation: $\delta_{\mathrm{t}}=\bar{\omega}+\bar{\beta} \delta_{t-1}+\bar{\alpha} \frac{1}{\mathrm{q}} \sum_{\mathrm{j}=1}^{\mathrm{q}}\left|\mathrm{u}_{\mathrm{t}-\mathrm{i}}-\mathrm{v}_{\mathrm{t}-\mathrm{i}}\right|$. Finally, we considered time-varying dependence for the symmetrized Joe-Clayton $\quad(\mathrm{SCJ}) \quad$ copula by assuming that $\quad \tau_{\mathrm{t}}^{\mathrm{U}}=\Lambda_{2}\left(\psi_{0, \mathrm{U}}+\psi_{1, \mathrm{U}} \tau_{\mathrm{t}-1}^{\mathrm{U}}+\left.\psi_{2, \mathrm{U}} \frac{1}{\mathrm{q}} \Sigma_{\mathrm{j}=1}^{\mathrm{q}}\right|_{\mathrm{t}-\mathrm{i}}-\mathrm{v}_{\mathrm{t}-\mathrm{i}} \mid\right) \quad$ and $\tau_{\mathrm{t}}^{\mathrm{L}}=\Lambda_{2}\left(\psi_{0, \mathrm{~L}}+\psi_{1, \mathrm{~L}} \tau_{\mathrm{t}-1}^{\mathrm{L}}+\psi_{2, \mathrm{~L}} \frac{1}{\mathrm{q}} \sum_{\mathrm{j}=1}^{\mathrm{q}}\left|\mathrm{u}_{\mathrm{t}-\mathrm{i}}-\mathrm{v}_{\mathrm{t}-\mathrm{i}}\right|\right), \quad$ where $\Lambda_{2}(\mathrm{x})=1 /\left(1+\mathrm{e}^{-\mathrm{x}}\right)^{-1} \quad$ is the logistic transformation used to keep $\tau^{\mathrm{U}} \quad$ and $\quad \tau^{\mathrm{L}} \quad$ in $\quad(0,1)$ 
Figures 4.2 and 4.3, which display the benchmark bond and MSCI price dynamics for all ten countries considered in our analysis, show differences in the size and timing of price movements in debt markets and the financial sector that become especially relevant after the onset of the debt crisis at the end of 2009. Price volatility changed significantly around the period of the recent global financial crisis for the MSCI index and around the period of the European debt crisis for debt price benchmarks, to degrees that differed significantly across core and peripheral countries. A superficial inspection of the data shows that co-movement between debt and financial sectors was different across countries and also changed with the onset of the debt crisis. Table 4.2 reports descriptive statistics for bond and financial price returns computed on a continuous compounding basis. Average returns were similarly close to zero in all the debt and financial markets and standard deviations were larger for the financial markets than for the debt markets. Also, differences in maximum and minimum values show that price ranges were greater for financial markets than for debt markets and greater for peripheral countries than for core countries. Negative values for skewness were common across markets and countries, with the exception of the Belgian, Italian and Spanish debt markets. All return series showed fat tails; the kurtosis statistic took high values and the Jarque-Bera test strongly rejected the normality of the unconditional distribution for all the series. The Ljung-Box statistic indicated that some return series displayed temporal correlation, whereas the ARCH-Lagrange multiplier (ARCH-LM) statistic indicated that $\mathrm{ARCH}$ effects could be found in all the return series.

Finally, in order to take into account the effects of the European sovereign debt crisis on expected returns and on volatility, we considered a crisis dummy variable in the mean and the variance of the marginal models that identified sample periods for before (value set to 0) and after (value set to 1) the onset of the European sovereign debt crisis. The crucial point here was to determine when the European sovereign debt crisis started. Following Bhanot et al. (2014), we took this date to be November 2009, as this was when investors became concerned regarding the quality of Greek debt; this concern developed in response to the Greek government's revelation that its deficit amounted to $12.7 \%$ of gross domestic product and not the previously announced $6.7 \%$. The fact that the impact of the crisis was different across debt markets is likely to have affected the dependence relationships between markets. 
Figure 4.2: Time series plot of weekly sovereign bond price indices.
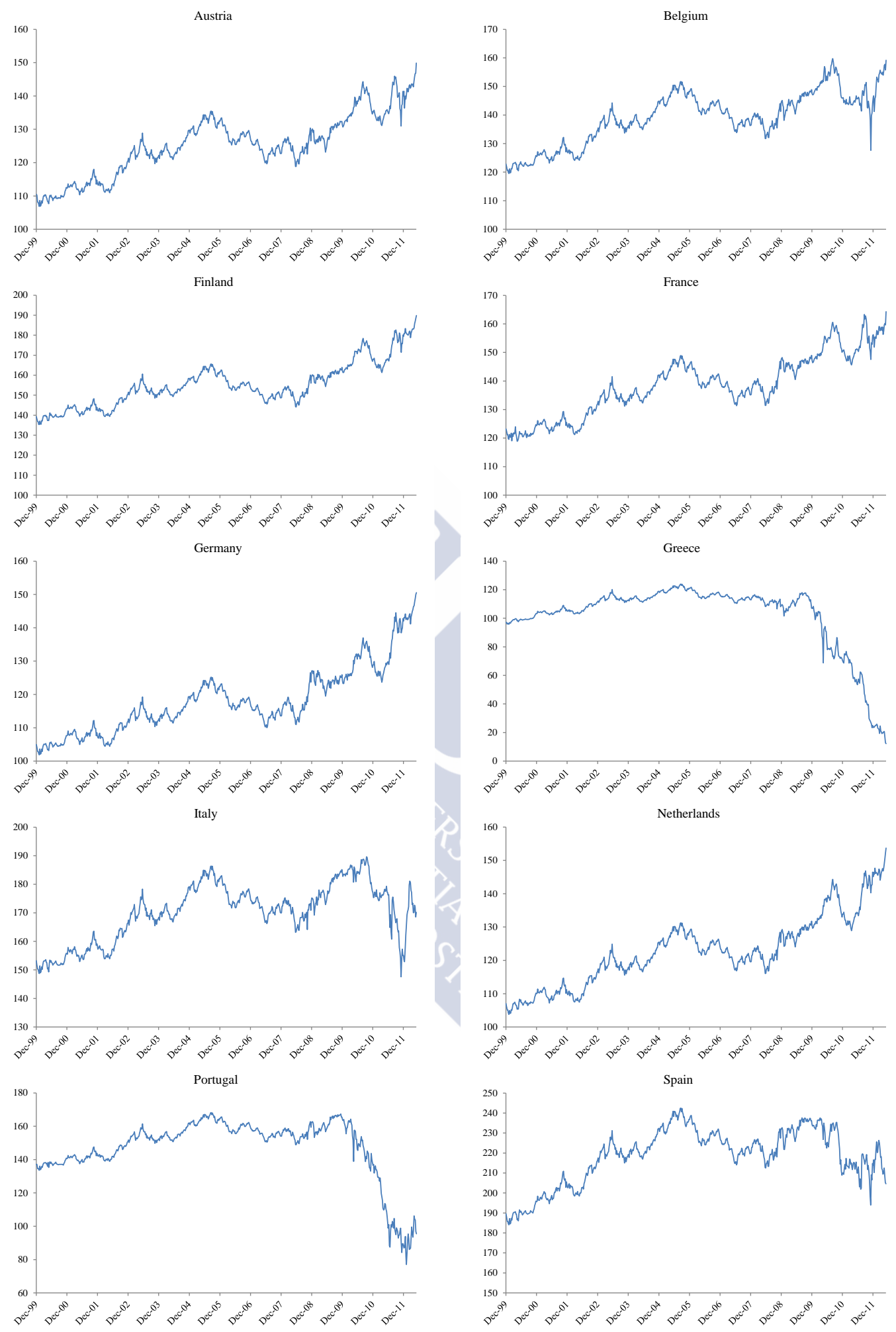
Chapter 4

Figure 4.3: Time series plot of weekly MSCI financial price indices.
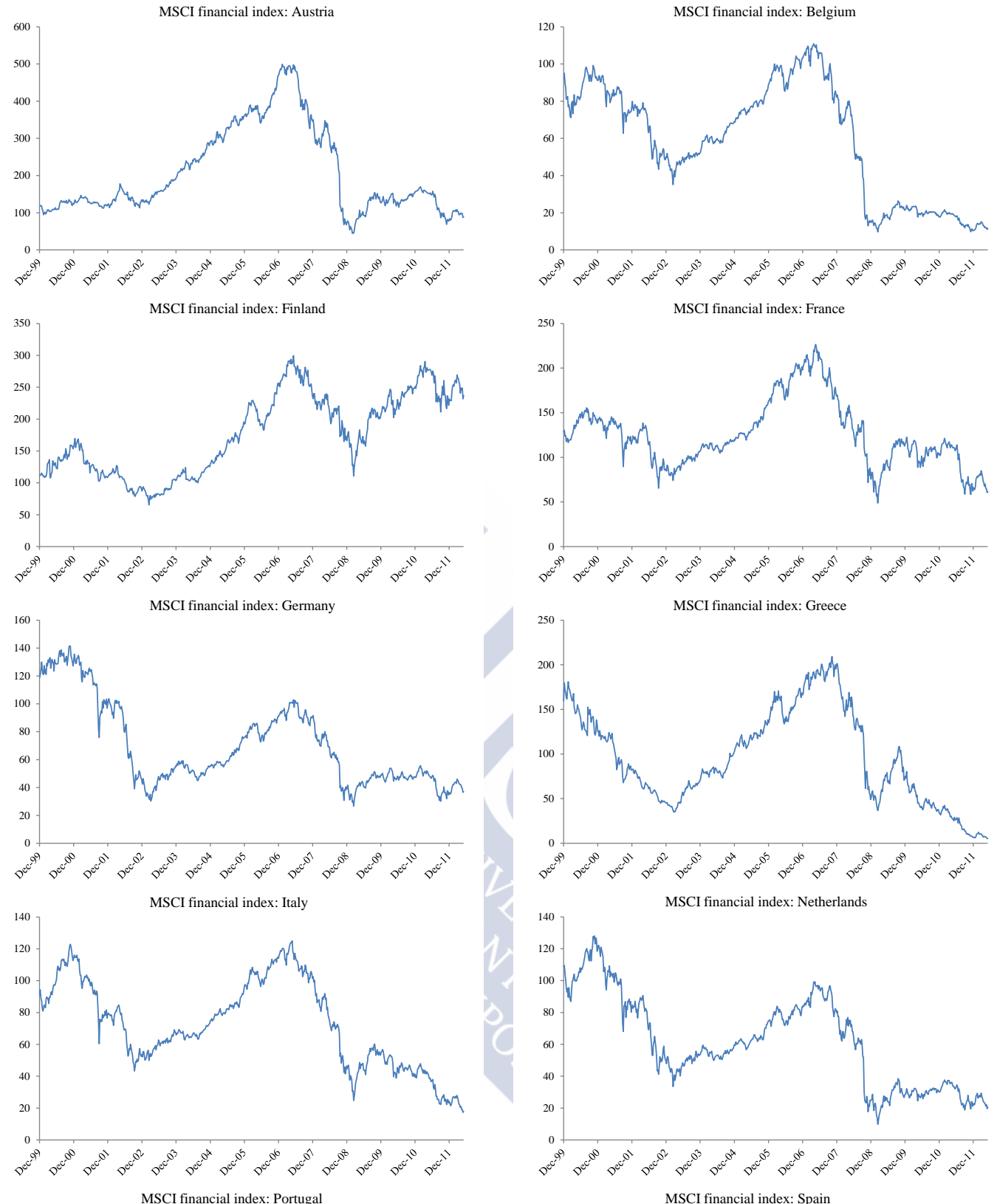

MSCI financial index: Greece
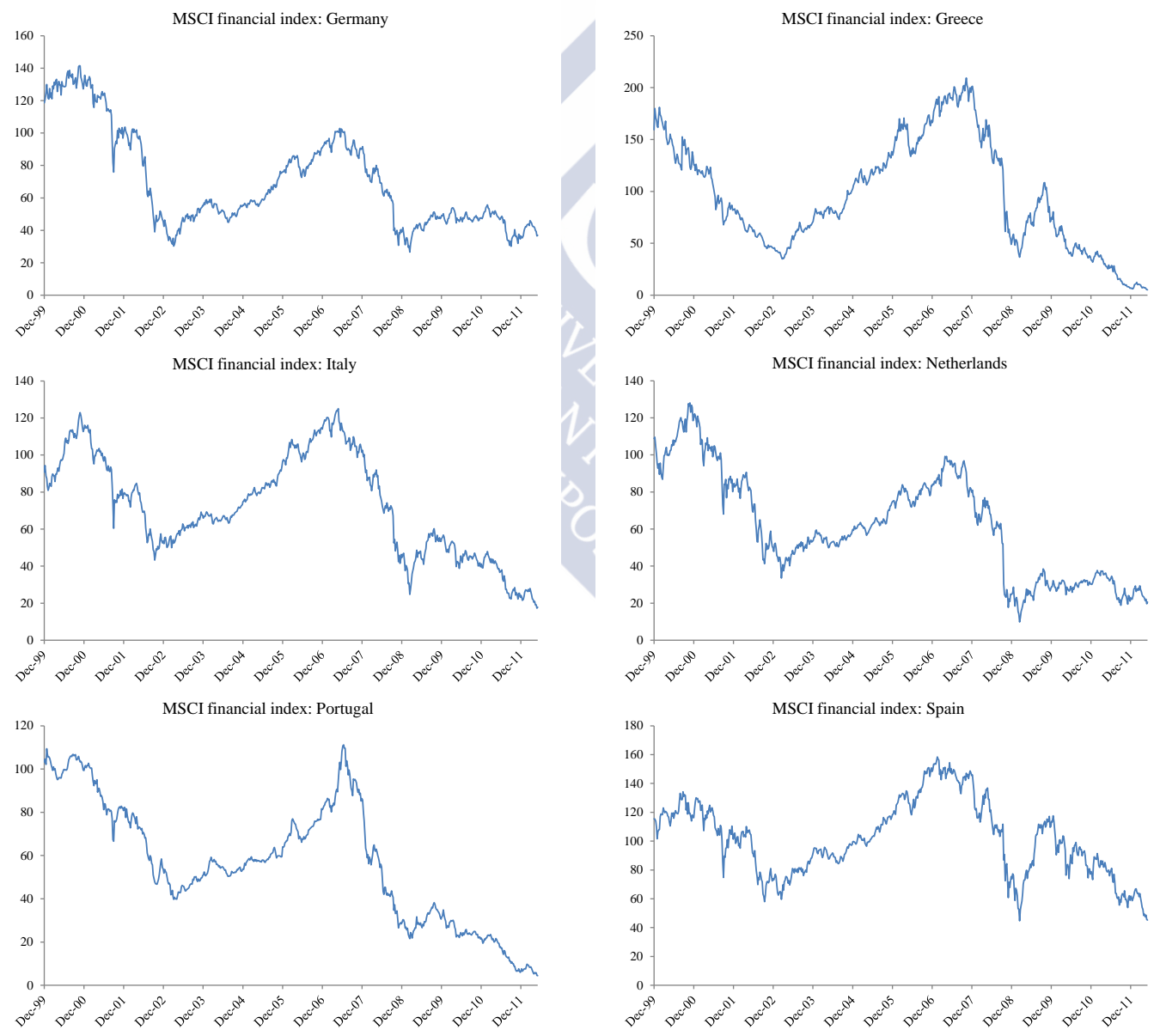

106 
A Vine-copula CoVaR approach to systemic sovereign debt risk for the financial...

Table 4.2: Descriptive statistics by country.

Panel A: Sovereign bond returns

\begin{tabular}{|c|c|c|c|c|c|c|c|c|c|c|}
\hline & $\mathrm{AT}$ & $\mathrm{BE}$ & FI & FR & $\mathrm{DE}$ & GR & IT & NL & $\mathrm{PT}$ & ES \\
\hline Mean & 0.000 & 0.000 & 0.000 & 0.000 & -0.003 & -0.003 & 0.000 & 0.001 & -0.001 & 0.000 \\
\hline SD & 0.008 & 0.009 & 0.007 & 0.008 & 0.022 & 0.032 & 0.009 & 0.008 & 0.019 & 0.010 \\
\hline Maximum & 0.046 & 0.091 & 0.026 & 0.039 & 0.135 & 0.293 & 0.082 & 0.027 & 0.127 & 0.079 \\
\hline Minimum & -0.036 & -0.079 & -0.029 & -0.032 & -0.142 & -0.287 & -0.043 & -0.029 & -0.137 & -0.038 \\
\hline Skewness & -0.250 & 0.217 & -0.178 & -0.065 & -1.487 & -1.428 & 0.917 & -0.191 & -0.253 & 1.195 \\
\hline Kurtosis & 6.352 & 23.528 & 3.873 & 4.446 & 16.739 & 44.200 & 14.986 & 3.722 & 20.809 & 14.658 \\
\hline$J-B$ & $310.6^{*}$ & $11400 .^{*}$ & $24.03^{*}$ & $56.98^{*}$ & $5343.9^{*}$ & 46122.* & $3975.8^{*}$ & $18.05^{*}$ & $8583.1^{*}$ & $3829.6^{*}$ \\
\hline $\mathrm{Q}(20)$ & $\begin{array}{l}62.252 \\
{[0.000]}\end{array}$ & $\begin{array}{l}74.578 \\
{[0.000]}\end{array}$ & $\begin{array}{l}26.007 \\
{[0.166]}\end{array}$ & $\begin{array}{l}26.139 \\
{[0.161]}\end{array}$ & $\begin{array}{l}24.095 \\
{[0.238]}\end{array}$ & $\begin{array}{l}128.27 \\
{[0.000]}\end{array}$ & $\begin{array}{l}51.085 \\
{[0.000]}\end{array}$ & $\begin{array}{l}24.729 \\
{[0.212]}\end{array}$ & $\begin{array}{l}106.01 \\
{[0.000]}\end{array}$ & $\begin{array}{l}29.106 \\
{[0.086]}\end{array}$ \\
\hline $\mathrm{ARCH}$ & $\begin{array}{l}10.417 \\
{[0.000]}\end{array}$ & $\begin{array}{l}15.579 \\
{[0.000]}\end{array}$ & $\begin{array}{r}5.390 \\
{[0.000]}\end{array}$ & $\begin{array}{r}8.485 \\
{[0.000]}\end{array}$ & $\begin{array}{r}6.275 \\
{[0.000]}\end{array}$ & $\begin{array}{l}17.654 \\
{[0.000]}\end{array}$ & $\begin{array}{r}4.750 \\
{[0.000]}\end{array}$ & $\begin{array}{r}5.330 \\
{[0.000]}\end{array}$ & $\begin{array}{r}9.929 \\
{[0.000]}\end{array}$ & $\begin{array}{l}10.900 \\
{[0.000]}\end{array}$ \\
\hline
\end{tabular}

Panel B: MSCI financial index returns

\begin{tabular}{lrllllllllr}
\hline & AT & \multicolumn{1}{l}{ BE } & FI & \multicolumn{1}{l}{ FR } & \multicolumn{1}{c}{ DE } & GR & IT & NL & PT & ES \\
\hline Mean & 0.000 & -0.003 & 0.001 & -0.001 & -0.002 & -0.005 & -0.002 & -0.003 & -0.005 & -0.001 \\
SD & 0.053 & 0.051 & 0.041 & 0.049 & 0.046 & 0.060 & 0.044 & 0.065 & 0.043 & 0.046 \\
Maximum & 0.240 & 0.193 & 0.158 & 0.195 & 0.230 & 0.255 & 0.228 & 0.238 & 0.181 & 0.181 \\
Minimum & -0.559 & -0.474 & -0.236 & -0.242 & -0.344 & -0.273 & -0.243 & -0.536 & -0.251 & -0.252 \\
Skewness & -2.253 & -1.657 & -0.643 & -0.361 & -0.578 & -0.236 & -0.904 & -1.382 & -0.744 & -0.657 \\
Kurtosis & 25.812 & 16.381 & 6.780 & 6.667 & 9.697 & 6.140 & 8.634 & 14.291 & 7.886 & 7.218 \\
JB & $14620 .^{*}$ & $5138.4^{*}$ & $431.21^{*}$ & $377.61^{*}$ & $1248.9^{*}$ & $272.6^{*}$ & $946.7^{*}$ & $3654.2^{*}$ & $705.3^{*}$ & $527.75^{*}$ \\
Q(20) & 57.325 & 51.874 & 23.286 & 29.874 & 31.819 & 34.312 & 40.792 & 53.924 & 41.351 & 31.039 \\
& {$[0.000]$} & {$[0.000]$} & {$[0.275]$} & {$[0.072]$} & {$[0.045]$} & {$[0.024]$} & {$[0.004]$} & {$[0.000]$} & {$[0.003]$} & {$[0.055]$} \\
ARCH & 6.349 & 6.527 & 4.879 & 12.142 & 5.958 & 8.175 & 7.166 & 8.644 & 11.381 & 8.497 \\
& {$[0.000]$} & {$[0.000]$} & {$[0.275]$} & {$[0.072]$} & {$[0.045]$} & {$[0.024]$} & {$[0.004]$} & {$[0.000]$} & {$[0.003]$} & {$[0.055]$} \\
\hline
\end{tabular}

Notes. Weekly data for the period 23 December 1999 to 25 May 2012. JB denotes the Jarque-Bera statistic for normality. $\mathrm{Q}(k)$ is the Ljung-Box statistic for serial correlation in squared returns computed with $k$ lags. ARCH denotes Engle's LM test for heteroskedasticity computed using 20 lags. An asterisk (*) indicates rejection of the null hypothesis at $5 \%$.

Table 4.3 provides information on the Pearson correlation between debt and financial returns for all the countries examined for the entire sample and for the periods before and after the onset of the European sovereign debt crisis (dated according to the dummy variable). It can be observed that linear dependence significantly changed for peripheral countries and Belgium, with correlation changing from negative to positive values; for these countries the diversification effect of sovereign debt dissipated with the onset of the sovereign debt crisis. However, for core countries we observed that the negative correlation values persisted after the onset of the crisis. The fact that the diversification role of sovereign debt was not changed in this case by the debt crisis has implications for CoVaR as we will discuss below. 
Table 4.3: Pearson correlation between financial returns and sovereign debt returns by country.

\begin{tabular}{lcccccccccc}
\hline & AT & BE & FI & FR & DE & GR & IT & NL & PT & ES \\
\hline All series & -0.07 & 0.00 & -0.23 & -0.21 & -0.36 & 0.27 & 0.14 & -0.24 & 0.20 & 0.04 \\
Before crisis onset & -0.13 & -0.19 & -0.19 & -0.25 & -0.30 & -0.04 & -0.13 & -0.20 & -0.07 & -0.21 \\
After crisis onset & 0.07 & 0.34 & -0.37 & -0.11 & -0.56 & 0.39 & 0.52 & -0.41 & 0.31 & 0.40 \\
\hline
\end{tabular}

\subsection{Empirical results}

\subsubsection{Marginal model results}

We estimated the marginal models in Eqs. (4.10)-(4.12) for sovereign debt and financial returns, taking the values of the parameters $\mathrm{p}, \mathrm{q}, \mathrm{r}$ and $\mathrm{m}$ as those that minimize the AIC values for possible lag values ranging from zero to a maximum of two. The evidence reported in Table 4.4 indicates that average sovereign debt returns displayed no serial correlation and were not affected by the outbreak of the debt crisis, except in Greece and Portugal, where impact was negative. The empirical volatility estimates confirm that volatility was persistent across different national markets and that leverage effects were hardly observed, except in Belgium, Finland and Italy. Our results also indicate that debt markets became more volatile with the onset of the debt crisis, as reflected by the positive significant effect of the dummy variable in the volatility equation. The estimates for the degrees of freedom and for the symmetry parameter of the skewed Student-t distribution confirm that error terms, as reported in Table 4.1, were not normal and were asymmetric, thereby providing evidence on the usefulness of the skewed Student-t distribution in modelling asymmetries in the marginals.

Table 4.5 reports the empirical results for the marginal distribution for the MSCI financial returns. Average financial returns showed no correlation and were negatively affected by the debt crisis, except in Austria, Finland, Germany and the Netherlands. Estimates indicate that volatility was persistent and that leverage effects were common across financial markets at the $10 \%$ significance level. Evidence was also found - consistent with the descriptive statistics reported in Table 4.1 - of asymmetries and fat tails in the financial return distributions. The last rows of Tables 4.4 and 4.5 show statistics for the goodness-of-fit of the marginal models, indicating that neither autocorrelation nor $\mathrm{ARCH}$ effects remained in the residuals. We also checked the adequacy of the skewed-t distribution model by testing the null 
hypothesis that the standardized model residuals were uniform $(0,1)$. To that end, we employed the well-known Kolmogorov-Smirnov, Cramér-von Mises and Anderson-Darling tests, which compare the empirical and theoretical distributions of the standardized residuals.

The $\mathrm{P}$ values for these tests (in the last three rows of Tables 4.2 and 4.3) indicate that the null hypothesis of correct specification of the distribution functions could not be rejected at the $5 \%$ significance level. Thus, evidence from the goodnessof-fit tests indicates that the marginal distribution models were not mis-specified, indicating, in turn, that the copula model could correctly capture dependence relationship between markets.

\subsubsection{Copula model results}

We estimated the bivariate copula models reported in Table 4.1 for each country for the MSCI financial and sovereign debt return pairs using, as observations, the probability integral transform of the standardized residuals from the marginal models. The best copula model was that which yielded the best AIC value corrected for small-sample bias. In the interest of brevity only graphical evidence is provided. ${ }^{12}$ Figure 4.4 shows the estimated parameter values, with parameter dynamics represented throughout the sample period for the best copula model fit. Our results indicate that static copulas offered a good fit for the core countries (Austria, Finland, France and Germany) for which tail independence existed between the financial sector and the domestic sovereign debt market. For the remaining two core countries (Belgium and the Netherlands), we found dependence to be time-varying and well captured by a Gaussian copula and therefore pointing to no evidence of tail dependence. The empirical estimates also showed that dependence was negative in both Belgium and Netherlands before the onset of the European sovereign debt crisis; thereafter, however, dependence continued to be negative for the Netherlands but turned positive for Belgium. Regarding the peripheral countries, our evidence for dependence pointed to a distinctive pattern in the pre- and post-onset sovereign debt crisis periods. Before the outbreak of the crisis, all the peripheral countries displayed time-varying negative dependence, as captured by the Student-t copula. The evidence for symmetric tail dependence indicated greater co-movement between the financial sector and sovereign debt markets for peripheral countries compared to the core countries showing evidence of tail independence. Moreover, this evidence

\footnotetext{
${ }^{12}$ Full information on copula model estimations is available on request.
} 
indicates that the safe-haven characteristics of sovereign debt differed widely across European countries. In considering the post-onset debt crisis period, dependence between financial sector and sovereign debt markets changed radically in the peripheral countries, turning this dependence positive and also pointing to positive tail dependence.

Table 4.4: Marginal distribution model. Parameter estimates for sovereign debt returns by country.

\begin{tabular}{|c|c|c|c|c|c|c|c|c|c|c|}
\hline & $\mathrm{AT}$ & $\mathrm{BE}$ & $\mathrm{FI}$ & FR & $\mathrm{DE}$ & GR & IT & NL & PT & $\mathrm{ES}$ \\
\hline \multicolumn{11}{|l|}{ Mean } \\
\hline$\phi_{0}$ & $\begin{array}{c}0.000 \\
(1.001)\end{array}$ & $\begin{array}{c}0.000 \\
(0.899)\end{array}$ & $\begin{array}{c}0.000 \\
(0.772)\end{array}$ & $\begin{array}{c}0.000 \\
(0.935)\end{array}$ & $\begin{array}{c}0.000 \\
(1.006)\end{array}$ & $\begin{array}{c}0.000 \\
(1.381)\end{array}$ & $\begin{array}{c}0.000 \\
(0.653)\end{array}$ & $\begin{array}{c}0.000 \\
(1.307)\end{array}$ & $\begin{array}{c}0.000 \\
(0.837)\end{array}$ & $\begin{array}{c}0.000 \\
(0.931)\end{array}$ \\
\hline Dummy & $\begin{array}{c}0.000 \\
(0.293)\end{array}$ & $\begin{array}{c}-0.001 \\
(-0.504)\end{array}$ & $\begin{array}{c}0.001 \\
(1.285)\end{array}$ & $\begin{array}{c}0.000 \\
(0.685)\end{array}$ & $\begin{array}{c}0.001 \\
(1.306)\end{array}$ & $\begin{array}{l}-0.012^{*} \\
(-4.304)\end{array}$ & $\begin{array}{c}-0.001 \\
(-1.133)\end{array}$ & $\begin{array}{c}0.001 \\
(1.392)\end{array}$ & $\begin{array}{l}-0.005^{*} \\
(-2.884)\end{array}$ & $\begin{array}{c}-0.002 \\
(-1.656)\end{array}$ \\
\hline \multicolumn{11}{|l|}{ Variance } \\
\hline$\omega$ & $\begin{array}{l}2.405^{*} \\
(2.023)\end{array}$ & $\begin{array}{l}3.632^{*} \\
(2.221)\end{array}$ & $\begin{array}{l}1.440^{*} \\
(2.663)\end{array}$ & $\begin{array}{c}2.407 \\
(1.679)\end{array}$ & $\begin{array}{l}1.934^{*} \\
(2.840)\end{array}$ & $\begin{array}{c}0.023 \\
(1.265)\end{array}$ & $\begin{array}{l}1.748^{*} \\
(2.838)\end{array}$ & $\begin{array}{l}2.088^{*} \\
(2.420)\end{array}$ & $\begin{array}{l}0.025^{*} \\
(2.615)\end{array}$ & $\begin{array}{c}2.428 \\
(1.950)\end{array}$ \\
\hline Dummy & $\begin{array}{l}0.000^{*} \\
(132.7)\end{array}$ & $\begin{array}{l}0.000^{*} \\
(181.5)\end{array}$ & $\begin{array}{l}0.000^{*} \\
(3.180)\end{array}$ & $\begin{array}{c}0.000^{*} \\
(7.253)\end{array}$ & $\begin{array}{l}0.000^{*} \\
(1.730)\end{array}$ & $\begin{array}{l}0.000^{*} \\
(2.812)\end{array}$ & $\begin{array}{c}0.000^{*} \\
(22.200)\end{array}$ & $\begin{array}{l}0.000^{*} \\
(6.970)\end{array}$ & $\begin{array}{l}0.000^{*} \\
(311.3)\end{array}$ & $\begin{array}{l}0.000^{*} \\
(154.4)\end{array}$ \\
\hline$\alpha_{1}$ & $\begin{array}{l}0.097^{*} \\
(3.325)\end{array}$ & $\begin{array}{c}0.008 \\
(0.521)\end{array}$ & $\begin{array}{l}0.104^{*} \\
(4.654)\end{array}$ & $\begin{array}{l}-0.063 \\
(-1.234)\end{array}$ & $\begin{array}{c}0.016 \\
(0.409)\end{array}$ & $\begin{array}{c}-0.046 \\
(-1.764)\end{array}$ & $\begin{array}{c}-0.033^{*} \\
(-2.630)\end{array}$ & $\begin{array}{l}-0.017 \\
(-0.654)\end{array}$ & $\begin{array}{c}0.052 \\
(1.406)\end{array}$ & $\begin{array}{c}0.041 \\
(1.501)\end{array}$ \\
\hline$\beta_{1}$ & $\begin{array}{c}0.869^{*} \\
(25.370)\end{array}$ & $\begin{array}{c}0.808^{*} \\
(19.170)\end{array}$ & $\begin{array}{c}0.899^{*} \\
(50.600)\end{array}$ & $\begin{array}{c}0.839^{*} \\
(17.610)\end{array}$ & $\begin{array}{c}0.897^{*} \\
(46.250)\end{array}$ & $\begin{array}{c}0.850^{*} \\
(33.570)\end{array}$ & $\begin{array}{c}0.904^{*} \\
(47.090)\end{array}$ & $\begin{array}{c}0.867^{*} \\
(32.940)\end{array}$ & $\begin{array}{c}0.873^{*} \\
(27.340)\end{array}$ & $\begin{array}{c}0.875^{*} \\
(19.410)\end{array}$ \\
\hline$\lambda$ & $\begin{array}{c}-0.019 \\
(-0.484)\end{array}$ & $\begin{array}{l}0.206^{*} \\
(2.221)\end{array}$ & $\begin{array}{l}-0.062^{*} \\
(-2.019)\end{array}$ & $\begin{array}{c}0.130 \\
(1.957)\end{array}$ & $\begin{array}{c}-0.008 \\
(-0.146)\end{array}$ & $\begin{array}{c}0.146 \\
(0.648)\end{array}$ & $\begin{array}{l}0.262^{*} \\
(3.046)\end{array}$ & $\begin{array}{c}0.059 \\
(1.126)\end{array}$ & $\begin{array}{c}0.076 \\
(1.293)\end{array}$ & $\begin{array}{c}0.090 \\
(1.276)\end{array}$ \\
\hline Asymetry & $\begin{array}{l}-0.143^{*} \\
(-2.859)\end{array}$ & $\begin{array}{l}-0.144^{*} \\
(-2.807)\end{array}$ & $\begin{array}{l}-0.117^{*} \\
(-2.597)\end{array}$ & $\begin{array}{l}-0.113^{*} \\
(-2.391) \\
188795\end{array}$ & $\begin{array}{l}-0.112^{*} \\
(-2.396)\end{array}$ & $\begin{array}{c}-0.092 \\
(-1.649)\end{array}$ & $\begin{array}{c}-0.106^{*} \\
(-2.168)\end{array}$ & $\begin{array}{l}-0.108^{*} \\
(-2.166) \\
187.28 ?\end{array}$ & $\begin{array}{l}-0.131^{*} \\
(-2.453)\end{array}$ & $\begin{array}{l}-0.103^{*} \\
(-1.985)\end{array}$ \\
\hline Tail & $\begin{array}{l}13.610^{*} \\
(143.4)\end{array}$ & $\begin{array}{l}12.010^{*} \\
(35.56)\end{array}$ & $\begin{array}{c}303.905^{*} \\
(336.9)\end{array}$ & $(176.2)$ & $\begin{array}{c}238.863^{*} \\
(487.3)\end{array}$ & $\begin{array}{c}6.314 \\
(0.283)\end{array}$ & $\begin{array}{c}7.025^{*} \\
(77.62)\end{array}$ & $\begin{array}{c}* \\
(54.01)\end{array}$ & $\begin{array}{l}6.718^{*} \\
(34.63)\end{array}$ & $\begin{array}{l}9.315^{*} \\
(79.89)\end{array}$ \\
\hline LogLik & 2268.04 & 2245.77 & 2295.66 & 2256.66 & 2252.98 & 2080.39 & 2255.70 & 2280.07 & 2096.94 & 2208.03 \\
\hline LJ & $\begin{array}{l}17.297 \\
{[0.634]}\end{array}$ & $\begin{array}{l}21.544 \\
{[0.366]}\end{array}$ & $\begin{array}{l}15.271 \\
{[0.761]}\end{array}$ & $\begin{array}{l}19.709 \\
{[0.476]}\end{array}$ & $\begin{array}{l}17.380 \\
{[0.628]}\end{array}$ & $\begin{array}{l}23.790 \\
{[0.252]}\end{array}$ & $\begin{array}{l}21.467 \\
{[0.370]}\end{array}$ & $\begin{array}{l}16.569 \\
{[0.681]}\end{array}$ & $\begin{array}{l}17.922 \\
{[0.593]}\end{array}$ & $\begin{array}{l}20.713 \\
{[0.414]}\end{array}$ \\
\hline LJ 2 & $\begin{array}{l}25.371 \\
{[0.115]}\end{array}$ & $\begin{array}{l}23.941 \\
{[0.091]}\end{array}$ & $\begin{array}{l}25.771 \\
{[0.105]}\end{array}$ & $\begin{array}{l}26.038 \\
{[0.053]}\end{array}$ & $\begin{array}{l}23.612 \\
{[0.130]}\end{array}$ & $\begin{array}{l}24.936 \\
{[0.096]}\end{array}$ & $\begin{array}{l}24.873 \\
{[0.098]}\end{array}$ & $\begin{array}{l}20.870 \\
{[0.184]}\end{array}$ & $\begin{array}{l}24.303 \\
{[0.145]}\end{array}$ & $\begin{array}{l}21.002 \\
{[0.279]}\end{array}$ \\
\hline $\mathrm{ARCH}$ & $\begin{array}{c}1.182 \\
{[0.263]}\end{array}$ & $\begin{array}{c}1.139 \\
{[0.305]}\end{array}$ & $\begin{array}{c}1.193 \\
{[0.254]}\end{array}$ & $\begin{array}{c}1.082 \\
{[0.365]}\end{array}$ & $\begin{array}{c}1.269 \\
{[0.193]}\end{array}$ & $\begin{array}{c}1.305 \\
{[0.168]}\end{array}$ & $\begin{array}{c}1.347 \\
{[0.143]}\end{array}$ & $\begin{array}{c}0.957 \\
{[0.514]}\end{array}$ & $\begin{array}{c}0.961 \\
{[0.515]}\end{array}$ & $\begin{array}{c}1.021 \\
{[0.435]}\end{array}$ \\
\hline K-S & {$[0.470]$} & {$[0.466]$} & {$[0.390]$} & {$[0.692]$} & {$[0.507]$} & [0.859] & {$[0.951]$} & [0.893] & {$[0.732]$} & {$[0.821]$} \\
\hline C-vM & {$[0.855]$} & {$[0.734]$} & {$[0.560]$} & {$[0.826]$} & {$[0.685]$} & {$[0.750]$} & {$[0.962]$} & {$[0.921]$} & {$[0.876]$} & {$[0.870]$} \\
\hline$A-D$ & [0.928] & [0.881] & {$[0.637]$} & {$[0.884]$} & {$[0.744]$} & [0.784] & {$[0.986]$} & {$[0.918]$} & {$[0.952]$} & {$[0.944]$} \\
\hline
\end{tabular}

Notes. The table presents the maximum likelihood estimates and z statistics (in parentheses) for the parameters of the marginal models described in Eqs. (4.10)-(4.12) for sovereign debt returns. LogLik is the log-likelihood value. LJ represents the Ljung-Box statistic for serial correlation in the residual model calculated with 20 lags. LJ ${ }^{2}$ represents the Ljung-Box statistic for serial correlation in the squared residual model calculated with 20 lags. $\mathrm{ARCH}$ is Engle's LM test for the ARCH effect in residuals up to 20th order. K-S, C-vM and A-D denote the Kolmogorov-Smirnov, Cramér-von Mises and Anderson-Darling tests for adequacy of the skewed-t distribution model. $\mathrm{P}$ values (in square brackets) below .05 indicate rejection of the null hypothesis. An asterisk $(*)$ indicates significance at $5 \%$. 
A Vine-copula CoVaR approach to systemic sovereign debt risk for the financial...

Table 4.5: Marginal distribution model. Parameter estimates for MSCI financial index returns by country.

\begin{tabular}{|c|c|c|c|c|c|c|c|c|c|c|}
\hline & $\mathrm{AT}$ & $\mathrm{BE}$ & FI & FR & $\mathrm{DE}$ & GR & $\mathrm{IT}$ & NL & $\mathrm{PT}$ & ES \\
\hline \multicolumn{11}{|l|}{ Mean } \\
\hline$\phi_{0}$ & $\begin{array}{c}0.002 \\
(1.816)\end{array}$ & $\begin{array}{l}-0.002^{*} \\
(-2.195)\end{array}$ & $\begin{array}{c}0.002 \\
(0.986)\end{array}$ & $\begin{array}{c}0.000 \\
(0.308)\end{array}$ & $\begin{array}{c}0.000 \\
(-0.193)\end{array}$ & $\begin{array}{c}0.001 \\
(0.285)\end{array}$ & $\begin{array}{c}0.000 \\
(0.413)\end{array}$ & $\begin{array}{c}0.000 \\
(-0.347)\end{array}$ & $\begin{array}{c}0.000 \\
(-0.075)\end{array}$ & $\begin{array}{c}0.001 \\
(0.436)\end{array}$ \\
\hline Dummy & $\begin{array}{c}-0.005 \\
(-1.466)\end{array}$ & $\begin{array}{l}-0.004^{*} \\
(-3.564)\end{array}$ & $\begin{array}{c}0.000 \\
(0.107)\end{array}$ & $\begin{array}{l}-0.006^{*} \\
(-5.385)\end{array}$ & $\begin{array}{c}-0.002 \\
(-1.667)\end{array}$ & $\begin{array}{l}-0.023^{*} \\
(-3.087)\end{array}$ & $\begin{array}{l}-0.008^{*} \\
(-2.495)\end{array}$ & $\begin{array}{c}-0.004 \\
(-1.293)\end{array}$ & $\begin{array}{l}-0.013^{*} \\
(-2.321)\end{array}$ & $\begin{array}{l}-0.010^{*} \\
(-9.237)\end{array}$ \\
\hline \multicolumn{11}{|l|}{ Variance } \\
\hline$\omega$ & $\begin{array}{l}0.311^{*} \\
(2.825)\end{array}$ & $\begin{array}{l}0.320^{*} \\
(3.934)\end{array}$ & $\begin{array}{c}1.387 \\
(1.520)\end{array}$ & $\begin{array}{l}0.470^{*} \\
(4.008)\end{array}$ & $\begin{array}{l}0.573^{*} \\
(2.354)\end{array}$ & $\begin{array}{l}0.812^{*} \\
(2.263)\end{array}$ & $\begin{array}{l}0.309^{*} \\
(3.375)\end{array}$ & $\begin{array}{l}0.413^{*} \\
(2.880)\end{array}$ & $\begin{array}{c}0.141 \\
(1.828)\end{array}$ & $\begin{array}{l}0.323^{*} \\
(3.952)\end{array}$ \\
\hline \multirow[t]{2}{*}{ Dummy } & $0.000^{*}$ & $0.000^{*}$ & $0.000^{*}$ & $0.000^{*}$ & $0.000^{*}$ & $0.000^{*}$ & $0.000^{*}$ & $0.000^{*}$ & $0.000^{*}$ & $0.000 *$ \\
\hline & $(24.48)$ & $(605.1)$ & $(-12.43)$ & $(2,126)$. & (298.1) & (2.449) & (413.9) & (221.8) & (3.438) & $(2,0)$ \\
\hline$\alpha_{1}$ & $\begin{array}{c}0.022 \\
(0.844)\end{array}$ & $\begin{array}{l}-0.131^{*} \\
(-4.408)\end{array}$ & $\begin{array}{c}-0.004 \\
(-0.089)\end{array}$ & $\begin{array}{l}-0.072^{*} \\
(-3.444)\end{array}$ & $\begin{array}{l}-0.046^{*} \\
(-4.043)\end{array}$ & $\begin{array}{c}0.039 \\
(1.754)\end{array}$ & $\begin{array}{c}-0.034 \\
(-1.055)\end{array}$ & $\begin{array}{c}0.001 \\
(0.045)\end{array}$ & $\begin{array}{c}0.040 \\
(1.283)\end{array}$ & $\begin{array}{l}-0.098^{*} \\
(-4.921)\end{array}$ \\
\hline \multirow[t]{2}{*}{$\beta_{1}$} & $0.886^{*}$ & $0.976^{*}$ & $0.825^{*}$ & $0.882^{*}$ & $0.877^{*}$ & $0.878^{*}$ & $0.899^{*}$ & $0.854^{*}$ & $0.865^{*}$ & $0.937^{*}$ \\
\hline & $\left(\begin{array}{c}(38.500 \\
)^{2}\end{array}\right.$ & $(106.40)$ & $(8.154)$ & (41.910) & $(19.550)$ & $(23.050)$ & $(19.280)$ & $(28.570)$ & $(39.270)$ & $(47.080)$ \\
\hline$\lambda$ & $\begin{array}{l}0.144^{*} \\
(3.577)\end{array}$ & $\begin{array}{l}0.249^{*} \\
(5.889)\end{array}$ & $\begin{array}{c}0.184 \\
(1.875)\end{array}$ & $\begin{array}{l}0.315^{*} \\
(6.663)\end{array}$ & $\begin{array}{l}0.240^{*} \\
(3.511)\end{array}$ & $\begin{array}{c}0.079^{*} \\
(2.299)\end{array}$ & $\begin{array}{c}0.172^{*} \\
(3.731)\end{array}$ & $\begin{array}{l}0.269^{*} \\
(4.423)\end{array}$ & $\begin{array}{l}0.201^{*} \\
(3.215)\end{array}$ & $\begin{array}{l}0.243^{*} \\
(8.007)\end{array}$ \\
\hline Asymetry & $\begin{array}{l}-0.119^{*} \\
(-2.217)\end{array}$ & $\begin{array}{l}-0.281^{*} \\
(-5.427)\end{array}$ & $\begin{array}{l}-0.181^{*} \\
(-2.633)\end{array}$ & $\begin{array}{l}-0.240^{*} \\
(-3.578)\end{array}$ & $\begin{array}{l}-0.206^{*} \\
(-3.034)\end{array}$ & $\begin{array}{c}0.002 \\
(0.030)\end{array}$ & $\begin{array}{l}-0.308^{*} \\
(-4.544)\end{array}$ & $\begin{array}{l}-0.240^{*} \\
(-3.651)\end{array}$ & $\begin{array}{c}-0.034 \\
(-0.618)\end{array}$ & $\begin{array}{l}-0.243^{*} \\
(-3.522)\end{array}$ \\
\hline Tail & $\begin{array}{c}9.932^{*} \\
(23.930\end{array}$ & $\begin{array}{c}6.607^{*} \\
(5.797)\end{array}$ & $\begin{array}{l}4.987^{*} \\
(5.387)\end{array}$ & $\begin{array}{c}12.954 \\
(1.792)\end{array}$ & $\begin{array}{c}10.209^{*} \\
(2.658)\end{array}$ & $\begin{array}{l}8.243^{*} \\
(3.297)\end{array}$ & $\begin{array}{c}9.074^{*} \\
(3.139)\end{array}$ & $\begin{array}{c}10.919^{*} \\
(2.872)\end{array}$ & $\begin{array}{l}4.108^{*} \\
(5.621)\end{array}$ & $\begin{array}{c}12.031^{*} \\
(2.223)\end{array}$ \\
\hline LogLik & 1228.30 & 1234.31 & 1241.51 & 1205.01 & 1224.85 & 1038.06 & 1302.31 & 1115.94 & 1344.75 & 1245.05 \\
\hline LJ & $\begin{array}{l}29.994 \\
{[0.070]}\end{array}$ & $\begin{array}{l}29.200 \\
{[0.084]}\end{array}$ & $\begin{array}{l}29.200 \\
{[0.848]}\end{array}$ & $\begin{array}{l}22.446 \\
{[0.317]}\end{array}$ & $\begin{array}{l}15.402 \\
{[0.753]}\end{array}$ & $\begin{array}{l}16.179 \\
{[0.705]}\end{array}$ & $\begin{array}{l}23.276 \\
{[0.275]}\end{array}$ & $\begin{array}{l}30.962 \\
{[0.056]}\end{array}$ & $\begin{array}{l}19.642 \\
{[0.353]}\end{array}$ & $\begin{array}{l}12.304 \\
{[0.905]}\end{array}$ \\
\hline LJ 2 & $\begin{array}{l}12.597 \\
{[0.815]}\end{array}$ & $\begin{array}{l}14.862 \\
{[0.671]}\end{array}$ & $\begin{array}{l}18.958 \\
{[0.394]}\end{array}$ & $\begin{array}{c}4.091 \\
{[1.000]}\end{array}$ & $\begin{array}{c}5.437 \\
{[0.998]}\end{array}$ & $\begin{array}{c}7.826 \\
{[0.981]}\end{array}$ & $\begin{array}{l}12.736 \\
{[0.807]}\end{array}$ & $\begin{array}{l}18.251 \\
{[0.439]}\end{array}$ & $\begin{array}{l}16.612 \\
{[0.550]}\end{array}$ & $\begin{array}{c}4.774 \\
{[0.999]}\end{array}$ \\
\hline $\mathrm{ARCH}$ & $\begin{array}{c}0.589 \\
{[0.921]}\end{array}$ & $\begin{array}{c}0.600 \\
{[0.914]}\end{array}$ & $\begin{array}{c}0.702 \\
{[0.826]}\end{array}$ & $\begin{array}{c}0.180 \\
{[1.000]}\end{array}$ & $\begin{array}{l}0.288 \\
{[0.999]}\end{array}$ & $\begin{array}{c}0.377 \\
{[0.994]}\end{array}$ & $\begin{array}{c}0.599 \\
{[0.915]}\end{array}$ & $\begin{array}{c}0.891 \\
{[0.599]}\end{array}$ & $\begin{array}{c}0.778 \\
{[0.742]}\end{array}$ & $\begin{array}{c}0.216 \\
{[1.000]}\end{array}$ \\
\hline K-S & {$[0.887]$} & {$[0.657]$} & {$[0.657]$} & {$[0.706]$} & {$[0.569]$} & {$[0.424]$} & [0.390] & {$[0.792]$} & {$[0.466]$} & {$[0.686]$} \\
\hline C-vM & {$[0.896]$} & {$[0.637]$} & {$[0.651]$} & {$[0.797]$} & {$[0.653]$} & [0.598] & {$[0.444]$} & {$[0.670]$} & {$[0.632]$} & {$[0.631]$} \\
\hline A-D & [0.939] & {$[0.741]$} & {$[0.786]$} & {$[0.859]$} & {$[0.783]$} & {$[0.683]$} & {$[0.541]$} & {$[0.723]$} & {$[0.715]$} & {$[0.731]$} \\
\hline
\end{tabular}

Notes. See Table 4.4 .

The results of the vine-copula model estimates are reported in Figures 4.5-4.7. Figure 4.5 displays the dynamics of the parameter estimates for the domestic financial system and sovereign Greek debt return pairs. For all the countries, timevarying copula models offered a better fit than the static copula models. We also observed a significant change in dependence for Belgium, Italy, the Netherlands, Portugal and Spain with the onset of the European sovereign debt crisis: the dependence parameter increased significantly, marking a change that was consistent with the relative importance of sovereign Greek debt for bank portfolios in those countries. However, for the other European countries (Austria, Finland, France and Germany), dependence was not significantly affected by the debt crisis. Only Austria, Finland and the Netherlands showed evidence of tail independence. 
Chapter 4

Figure 4.4: Times series plots for parameter estimates of the best copula fits between domestic financial systems and sovereign debt returns.
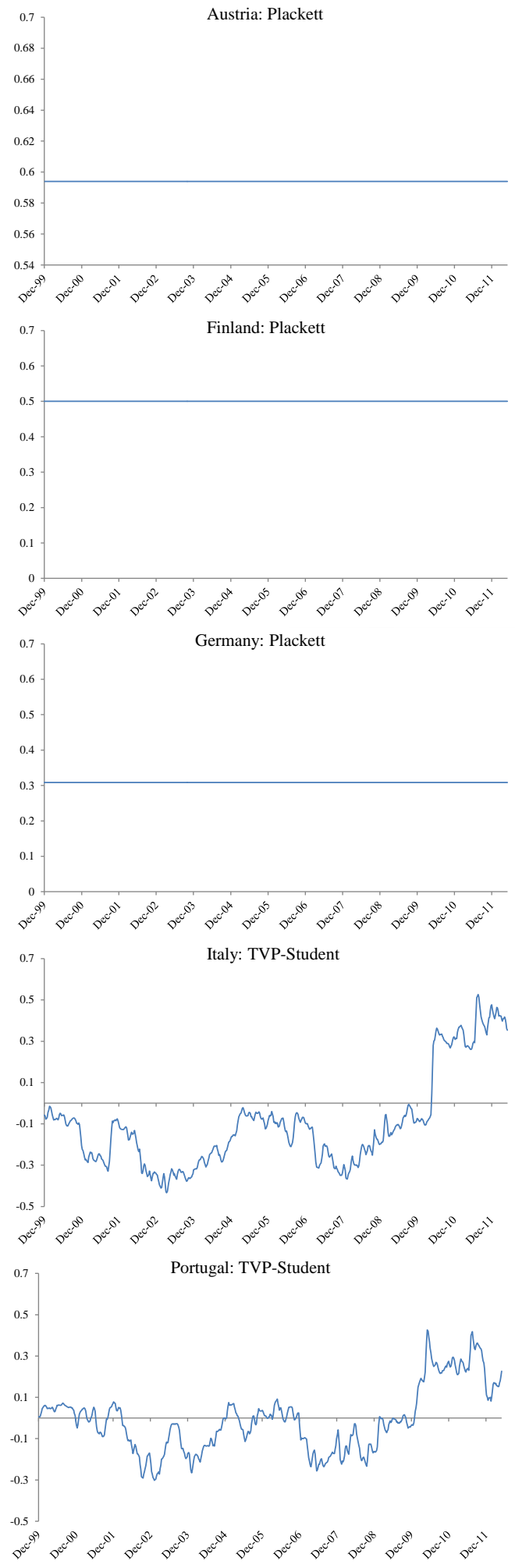

Belgium: TVP-Gaussian
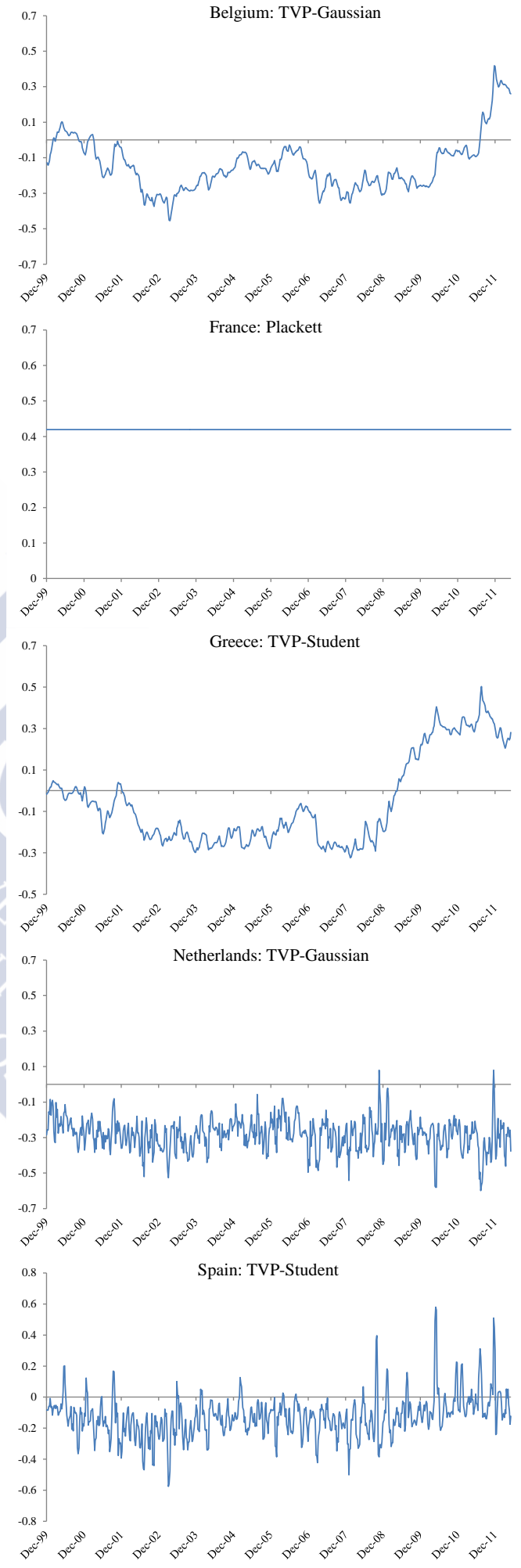

112 
Figure 4.6 displays the dynamics of the parameter estimates for the best copula model fit between the domestic sovereign debt and sovereign Greek debt return pairs. The evidence was conclusive in the period before the onset of the global financial crisis: all the debt markets strongly co-moved with the sovereign Greek debt market. However, thereafter - and mainly in the aftermath of the Greek debt crisis - European debt markets decoupled from the Greek market (markets in the core countries more so than in the peripheral countries), with dependence continuing to be positive, although less intense, in the peripheral countries. Of the core countries, Belgium was a particular case in that it exhibited high tail dependence at a specific times after the Greek crisis; a similar pattern was also observed for the Netherlands.

Figure 4.7 displays the dynamics of the parameter estimates for the best paircopula model for domestic financial and sovereign debt returns conditional on sovereign Greek debt returns: $c_{\mathrm{f}, \mathrm{d} \mid \mathrm{d}^{*}}\left(\mathrm{~F}_{\mathrm{f} \mid \mathrm{d}^{*}}\left(\mathrm{x}_{\mathrm{f}} \mid \mathrm{x}_{\mathrm{d}^{*}}\right), \mathrm{F}_{\mathrm{d} \mid \mathrm{d}^{*}}\left(\mathrm{x}_{\mathrm{d}} \mid \mathrm{x}_{\mathrm{d}^{*}}\right)\right)$. The empirical evidence is consistent with the evidence reported for the copula linking domestic financial and sovereign debt returns. Evidence on tail independence was found for all the core countries except Austria and Belgium. Dependence was static for Finland, France and the Netherlands but was time-varying for Austria, Belgium and Germany. Moreover, for Austria and Germany, dependence did not experience significant changes with the onset of the European debt crisis; in Belgium, dependence turned positive after 2011. Regarding peripheral countries, evidence of time-varying dependence was found for Italy and Spain and evidence of static dependence was found for Portugal. Dependence increased in the aftermath of the European debt crisis in Italy and, to a lesser extent, in Spain.

\subsubsection{Systemic risk results}

Using the best copula and vine-copula fits, we obtained the CoVaR for each time period following the two- and three-step procedures described above. We obtained CoVaR at the $95 \%$ confidence level $(\beta=0.05)$, conditional on the VaR for sovereign debt returns at the $95 \%$ confidence level $(\alpha=0.05$ or/and $\gamma=0.05) .{ }^{13}$ Below we present the results for $\mathrm{CoVaR}$ using bivariate copulas and then using vine copulas.

\footnotetext{
${ }^{13}$ Results at the $99 \%$ confidence level, which were consistent with the results reported here, are available on request.
} 
Figure 4.5: Times series plots for parameter estimates of the best copula fits between domestic financial systems and sovereign Greek debt returns.

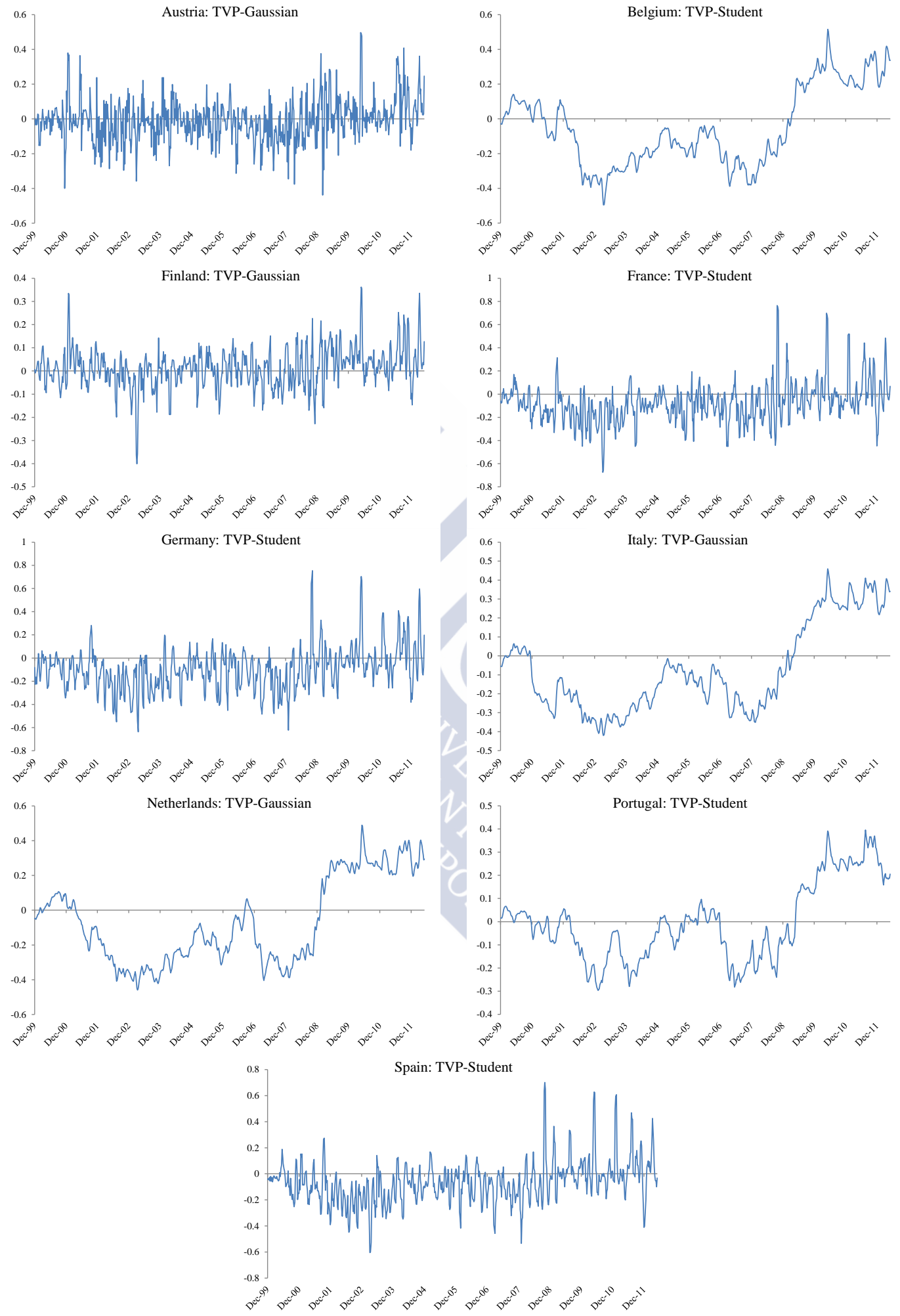


Figure 4.6: Times series plots for parameter estimates of the best copula fits between domestic sovereign and Greek sovereign debt returns.
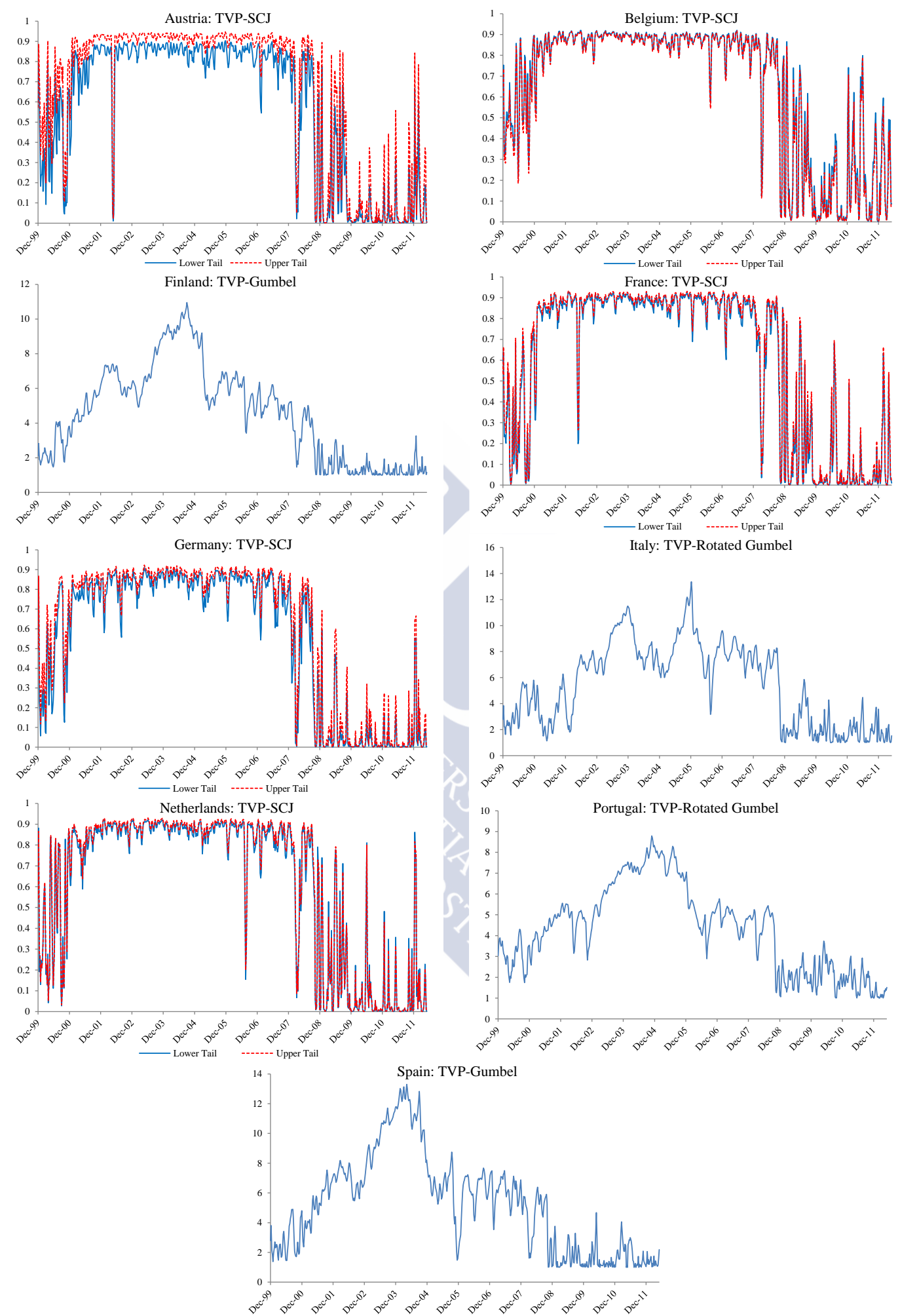
Figure 4.7: Times series plots for parameter estimates of the best copula fits between domestic financial systems and domestic sovereign debt returns conditional on sovereign Greek debt returns.
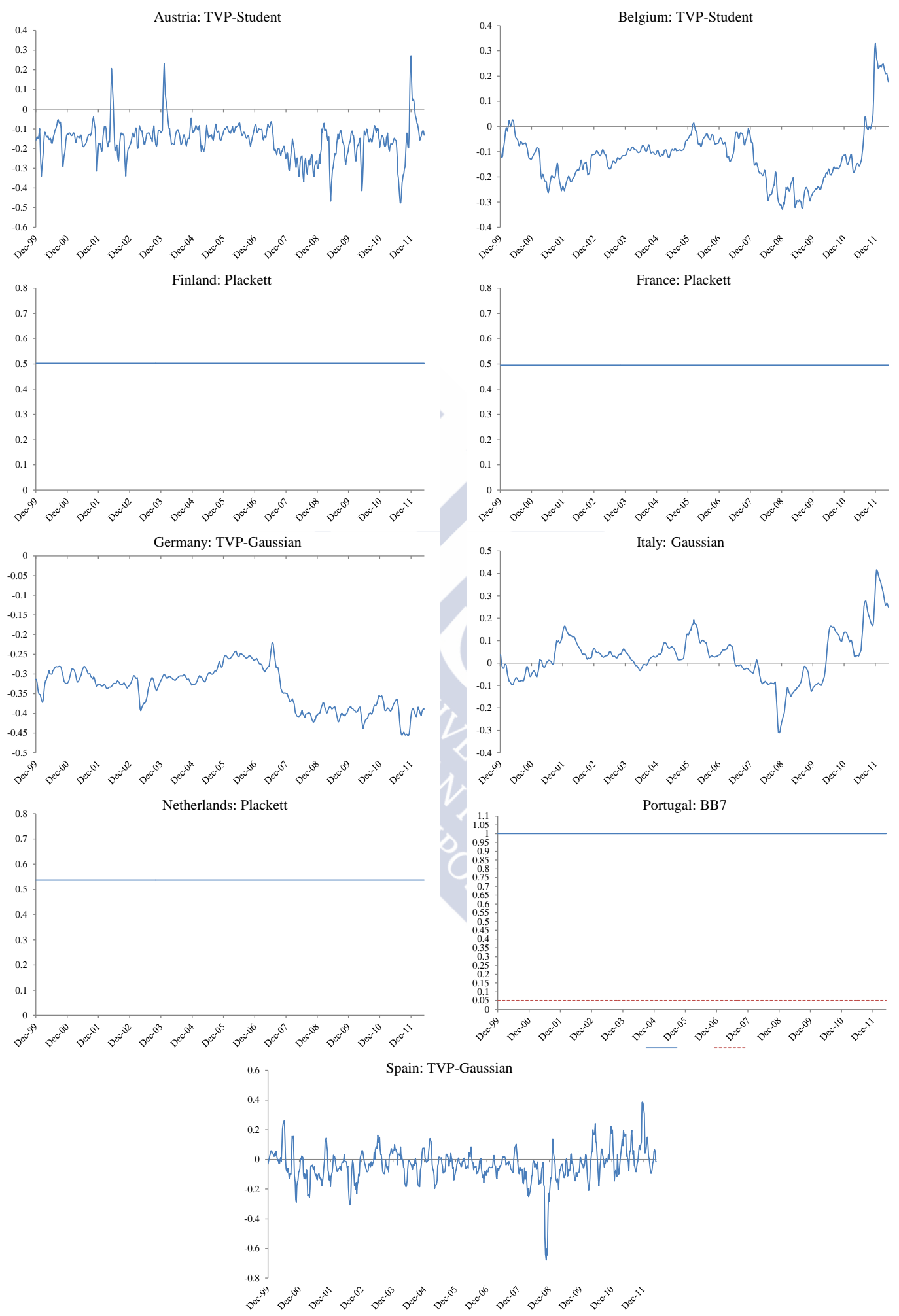
Figure 8 depicts the results for $\mathrm{CoVaR}$ dynamics throughout the sample period, with the post-onset financial and debt crisis periods indicated as shaded areas. For each figure representing the systemic risk of domestic sovereign debt for the financial systems for each country, we also included information on the financial system VaR so as to allow comparisons between VaR and CoVaR data. For each country in Table 6 , the first two rows provide information on average VaR and $\mathrm{CoVaR}$ for the entire sample and for the pre- and post-onset European sovereign debt crisis periods (dated according to the dummy variable). Our evidence shows that, in the pre-crisis period, domestic sovereign debt played a diversification role for domestic financial systems in the eurozone, as indicated by $\mathrm{CoVaR}$ figures that were greater than VaR figures. This was particularly relevant for the core countries where systemic risk reductions were greater than for the peripheral countries. This evidence confirms the diversification role played by sovereign debt across European countries in the pre-crisis period, with the intensity of this role varying across countries depending on the degree of dependence between sovereign and financial sector returns and the weight of sovereign debt in bank portfolios.

As our copula results show, dependence between domestic financial systems and sovereign debt markets changed in a different way across countries on the outbreak of the sovereign Greek debt crisis that had an impact on systemic risk. Figure 4.8 shows that sovereign debt for the core countries continued to play a diversification role for the financial system, given that CoVaR figures were, in general, greater than VaR figures; the only exception was Belgium, where, from the end of 2010, sovereign debt distress increased VaR. Table 4.6 shows, in fact, that average CoVaR figures were below the average VaR figures for all the core countries (except Belgium). However, for the peripheral countries, the opposite effect was observed: the systemic impact of sovereign debt increased considerably for Greece, Italy and Portugal, while remaining relatively stable for Spain. This evidence is consistent with the change in dependence observed for peripheral countries after the onset of the debt crisis; an increase in (positive) dependence swept away the diversification effects of domestic sovereign debt on domestic financial systems. 
Figure 4.8: $\operatorname{VaR}(\mathrm{f})$ and $\operatorname{CoVaR}(\mathrm{f} \mid \mathrm{d})$

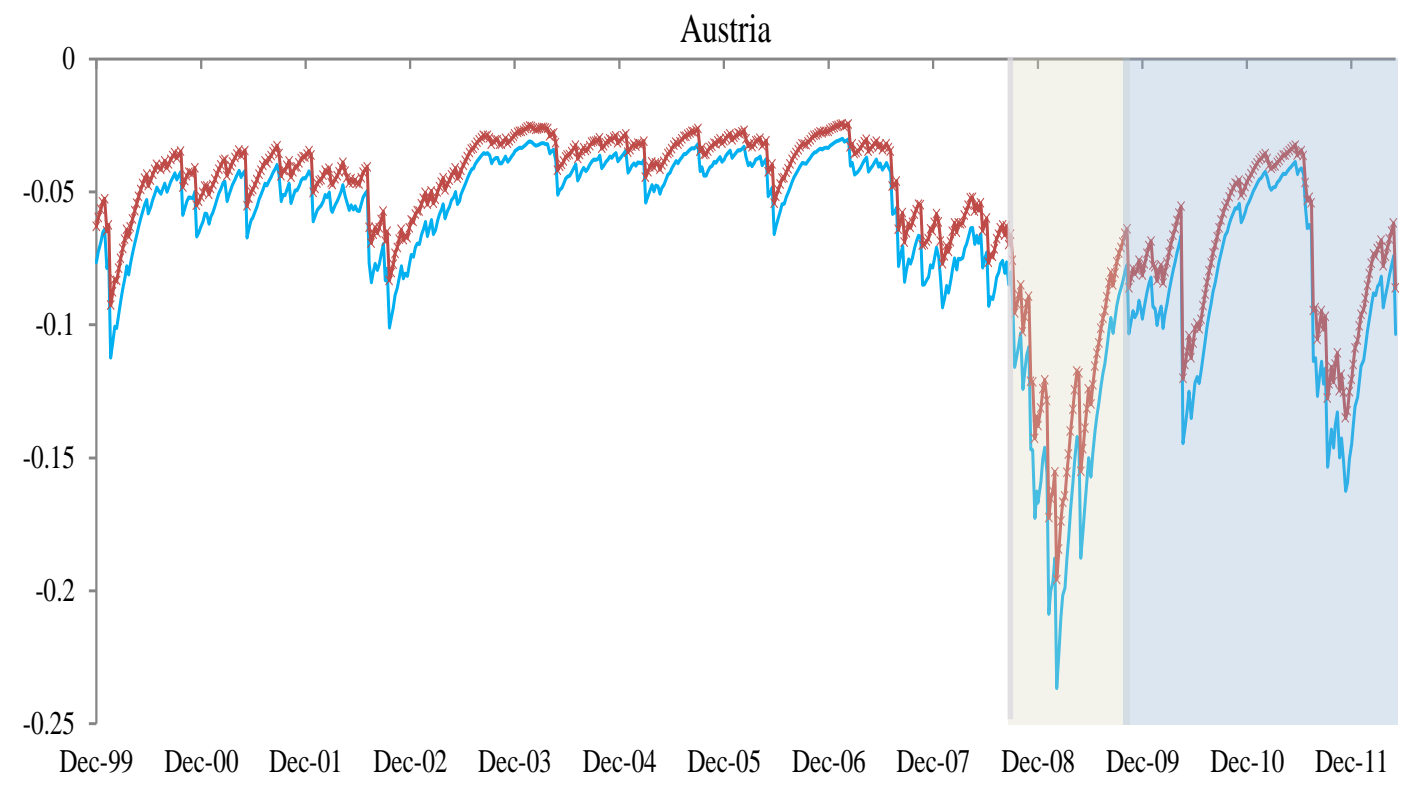

$\simeq \operatorname{VaR}(\mathrm{f}) \quad \longrightarrow \operatorname{CoVaR}(\mathrm{f} \mid \mathrm{d})$

Belgium

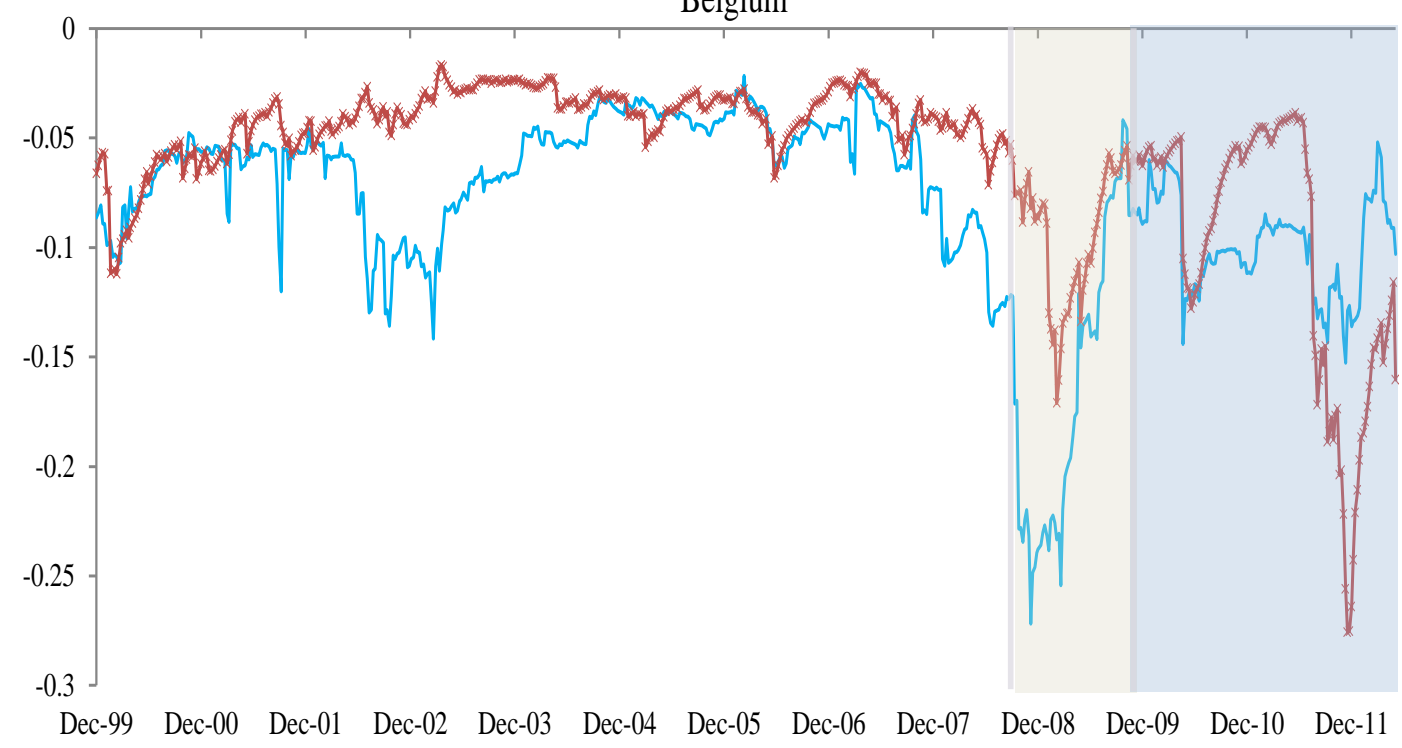

$\longrightarrow \operatorname{VaR}(\mathrm{f}) \multimap \mathrm{CoVaR}(\mathrm{f} \mid \mathrm{d})$ 
Figure 4.8: (Continued)

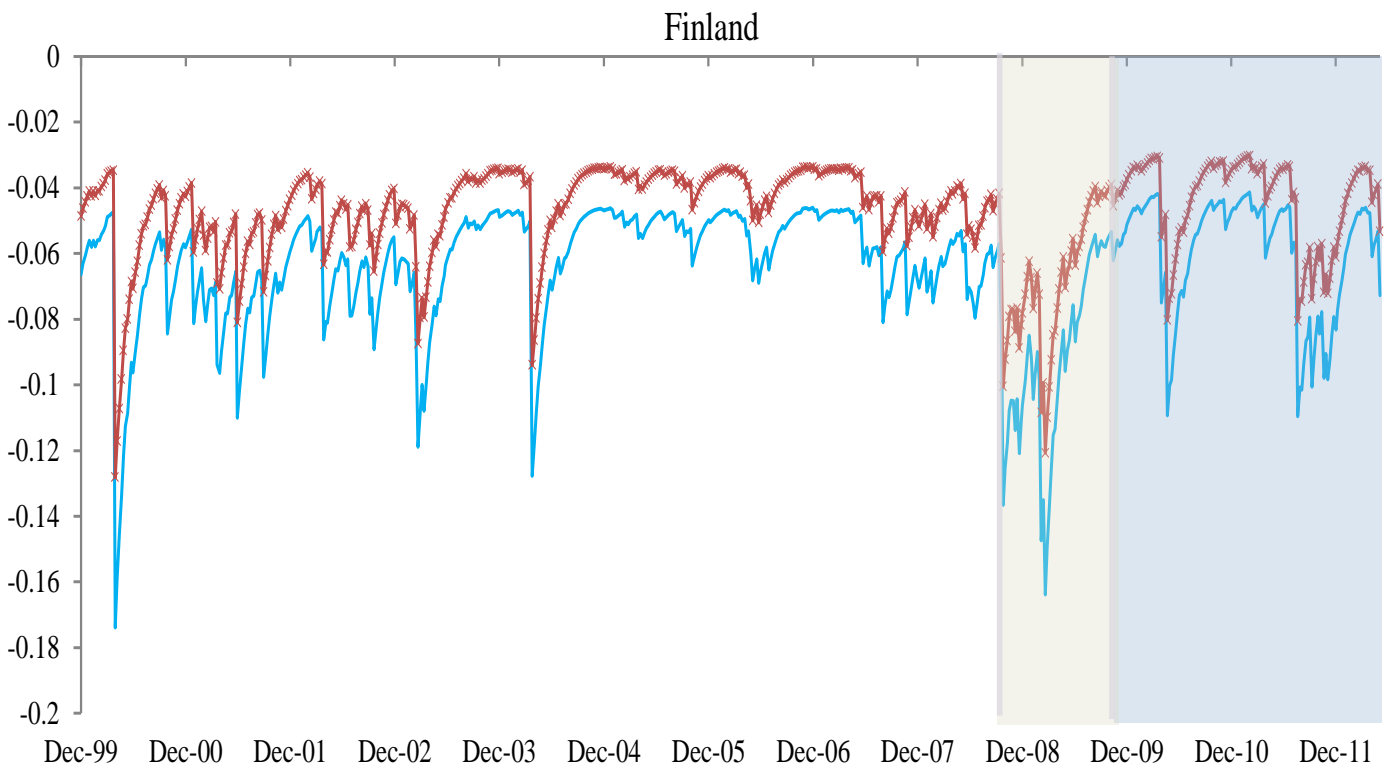

$\simeq \operatorname{VaR}(\mathrm{f}) \simeq \mathrm{CoVaR}(\mathrm{f} \mid \mathrm{d})$

France

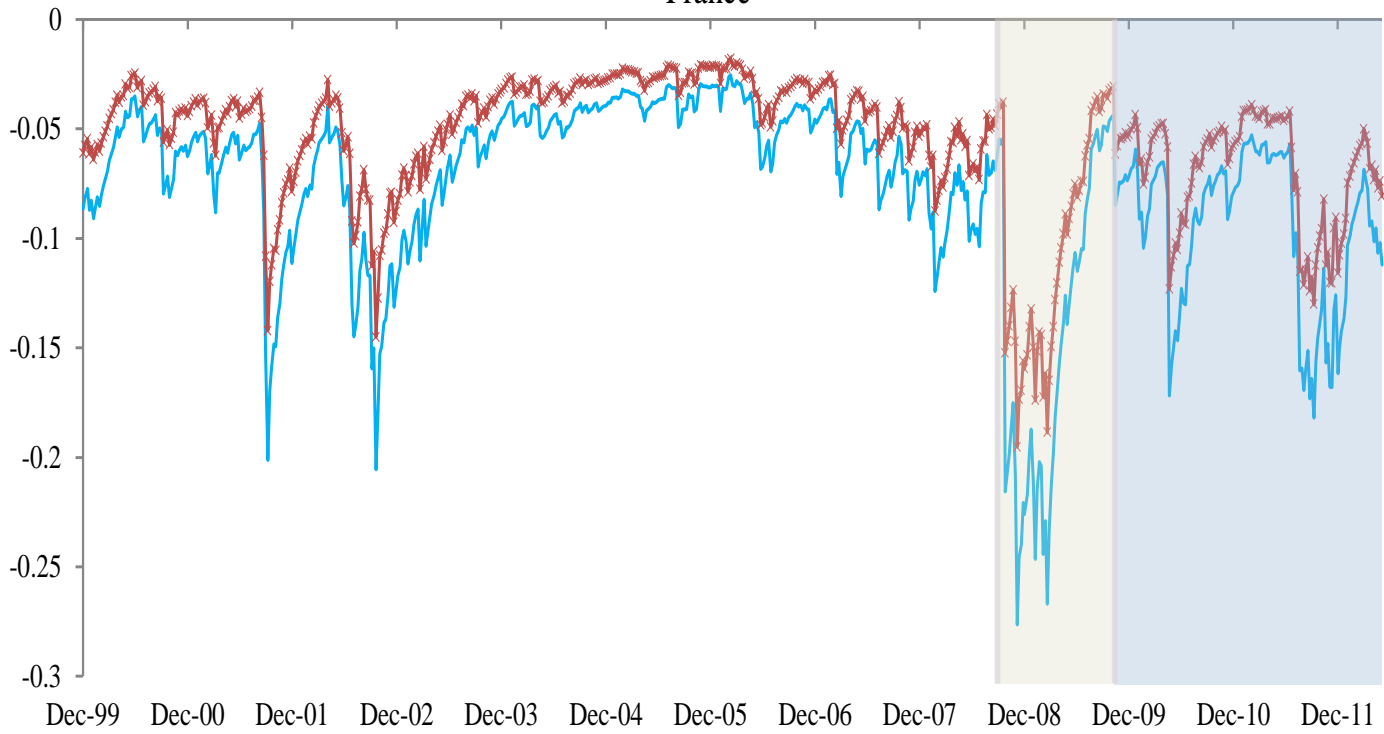

$\longrightarrow \operatorname{VaR}(\mathrm{f}) \longrightarrow \mathrm{CoVaR}(\mathrm{f} \mid \mathrm{d})$ 


\section{Figure 4.8: (Continued)}

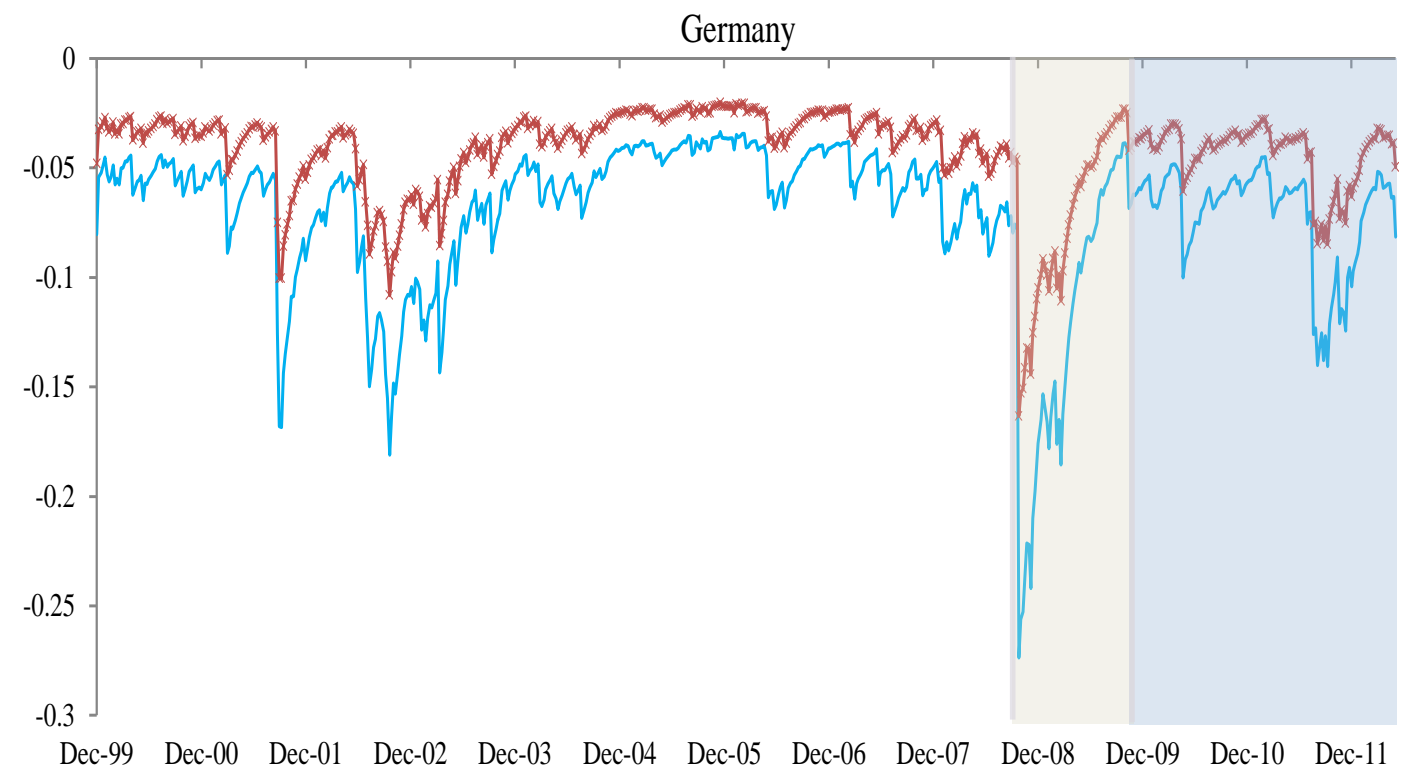

$\simeq \operatorname{VaR}(\mathrm{f}) \simeq \mathrm{CoVaR}(\mathrm{f} \mid \mathrm{d})$

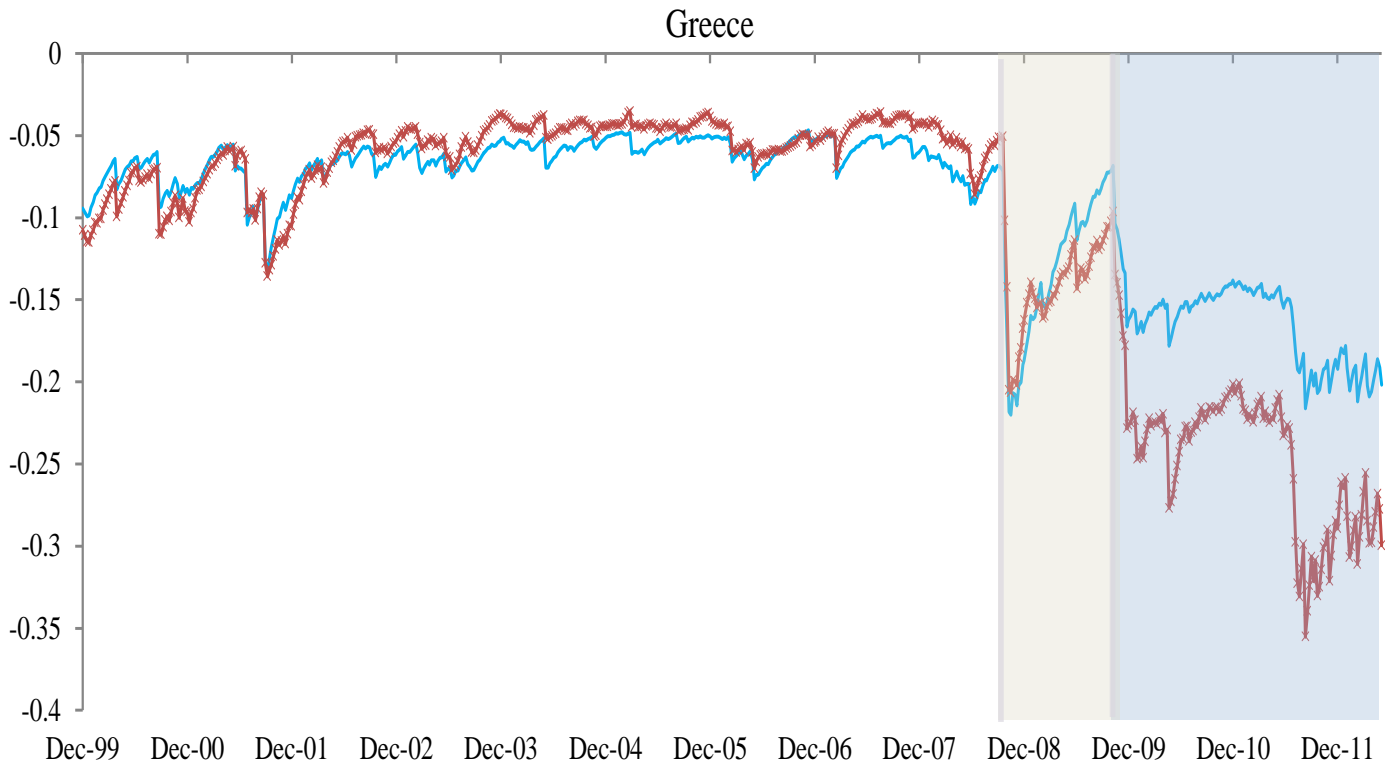

$\longrightarrow \operatorname{VaR}(\mathrm{f}) \multimap \mathrm{CoVaR}(\mathrm{f} \mid \mathrm{d})$ 
Figure 4.8: (Continued)

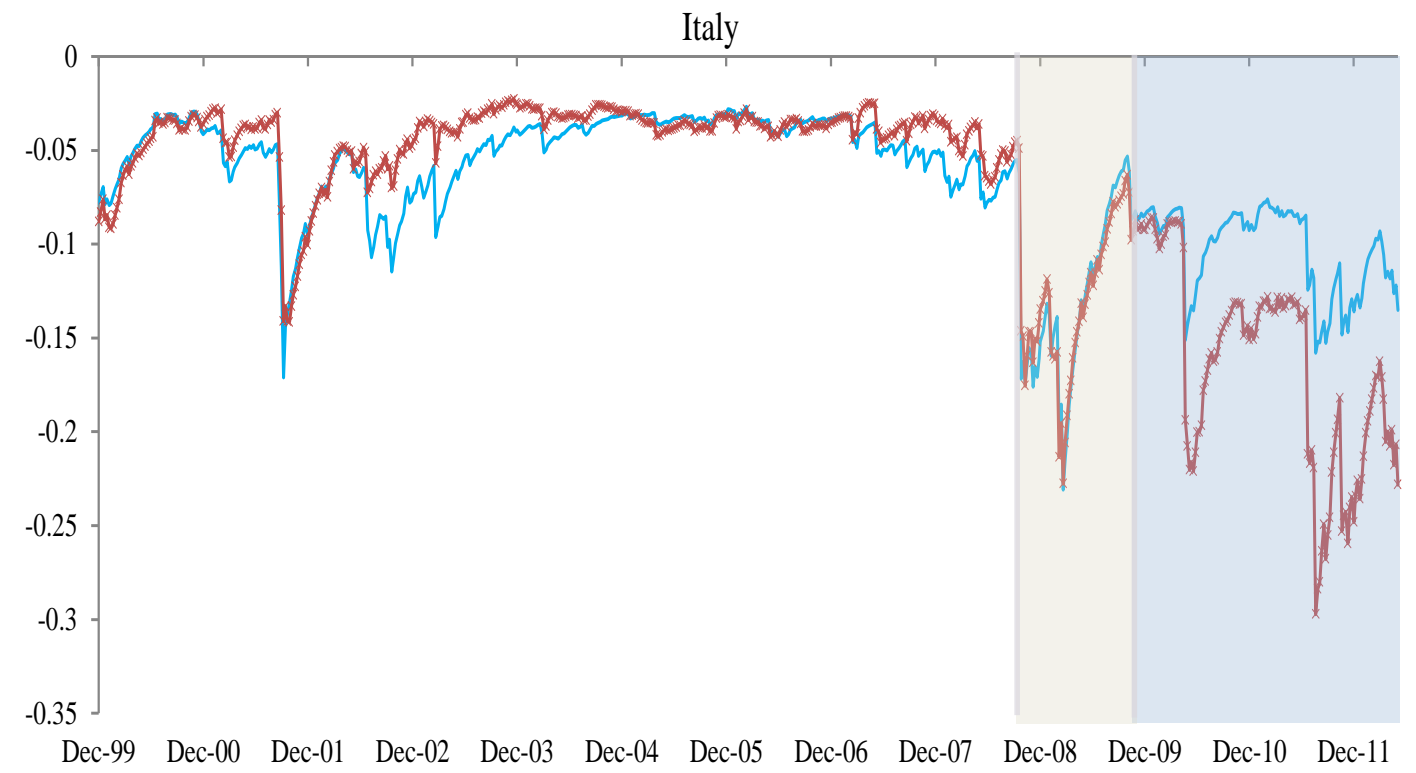

$\simeq \operatorname{VaR}(\mathrm{f}) \simeq \mathrm{CoVaR}(\mathrm{f} \mid \mathrm{d})$

Netherlands

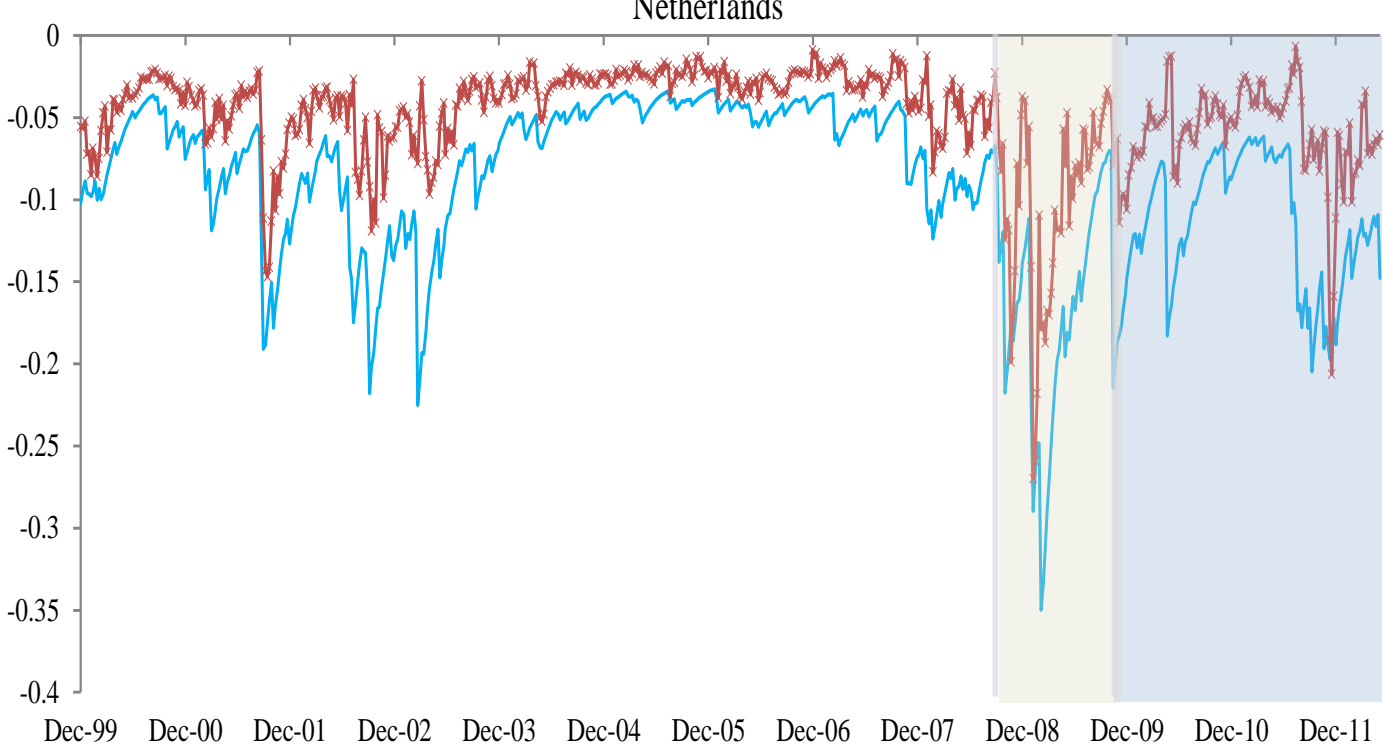

$\longrightarrow \operatorname{VaR}(\mathrm{f}) \multimap \mathrm{CoVaR}(\mathrm{f} \mid \mathrm{d})$ 


\section{Figure 4.8: (Continued)}

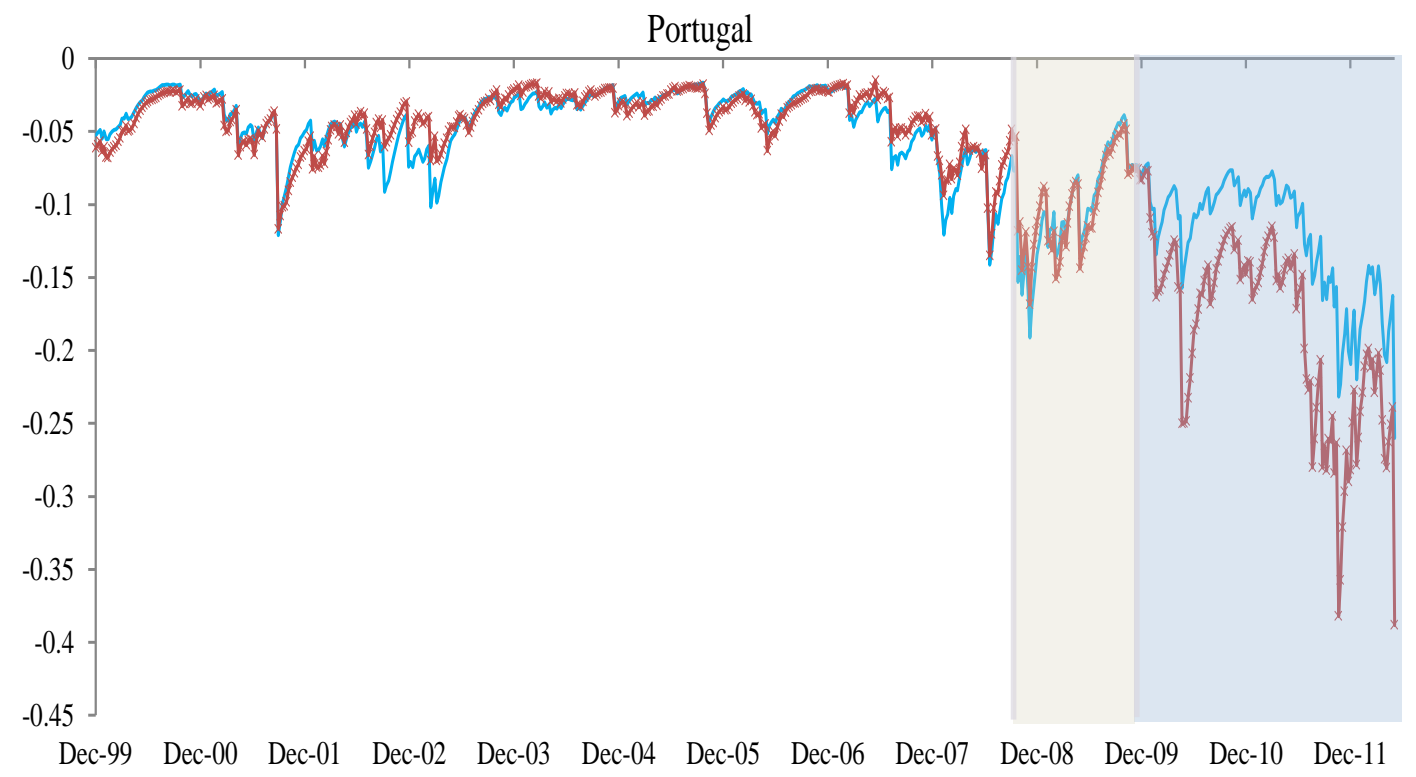

$\simeq \operatorname{VaR}(\mathrm{f}) \longrightarrow \mathrm{CoVaR}(\mathrm{f} \mid \mathrm{d})$

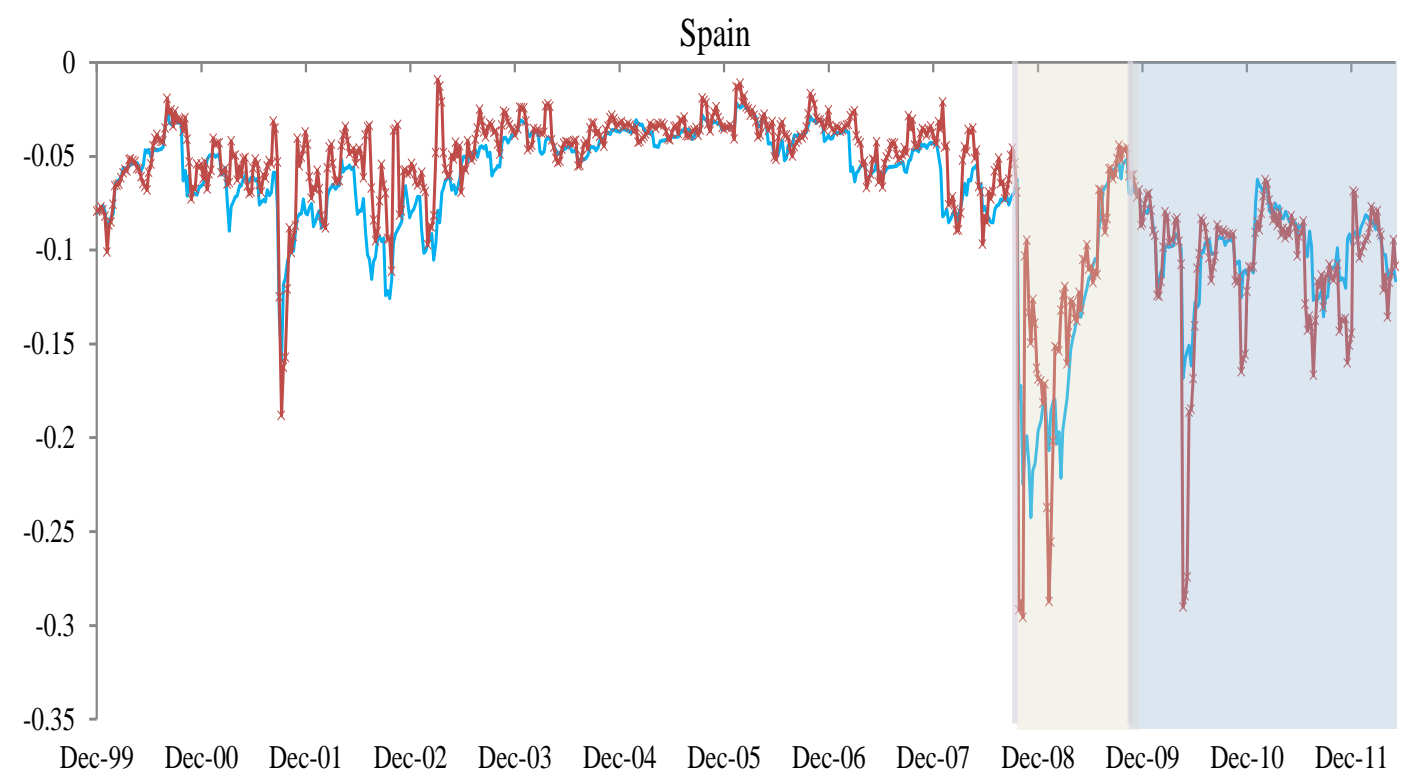

$\longrightarrow \operatorname{VaR}(\mathrm{f}) \multimap \mathrm{CoVaR}(\mathrm{f} \mid \mathrm{d})$ 
A Vine-copula CoVaR approach to systemic sovereign debt risk for the financial...

Table 4.6: CoVaR results.

\begin{tabular}{|c|c|c|c|c|c|c|c|}
\hline & & \multicolumn{2}{|c|}{ All Series } & \multicolumn{2}{|c|}{ Before Crisis Onset } & \multicolumn{2}{|c|}{ After Crisis Onset } \\
\hline & & Mean & $\mathrm{SD}$ & Mean & $\mathrm{SD}$ & Mean & $\mathrm{SD}$ \\
\hline \multirow[t]{5}{*}{ Austria } & $\operatorname{VaR}(\mathrm{f})$ & -0.068 & $(0.037)$ & -0.063 & $(0.036)$ & -0.088 & $(0.034)$ \\
\hline & $\mathrm{CoVaR}(\mathrm{f} \mid \mathrm{d})$ & -0.056 & $(0.030)$ & -0.052 & $(0.029)$ & -0.074 & $(0.028)$ \\
\hline & $\operatorname{CoVaR}\left(\mathrm{f} \mid \mathrm{d}=0.05, \mathrm{~d}^{*}\right)$ & -0.057 & $(0.031)$ & -0.052 & $(0.027)$ & -0.076 & $(0.037)$ \\
\hline & $\operatorname{CoVaR}\left(\mathrm{f} \mid \mathrm{d}, \mathrm{d}^{*}=0.05\right)$ & -0.061 & $(0.037)$ & -0.055 & $(0.033)$ & -0.085 & $(0.043)$ \\
\hline & $\operatorname{CoVaR}\left(\mathrm{f} \mid \mathrm{d}=0.05, \mathrm{~d}^{*}=0.05\right)$ & -0.058 & $(0.035)$ & -0.052 & $(0.032)$ & -0.081 & $(0.038)$ \\
\hline \multirow[t]{5}{*}{ Belgium } & $\operatorname{VaR}(\mathrm{f})$ & -0.080 & $(0.043)$ & -0.075 & $(0.046)$ & -0.099 & $(0.021)$ \\
\hline & $\operatorname{CoVaR}(\mathrm{f} \mid \mathrm{d})$ & -0.059 & $(0.041)$ & -0.048 & $(0.025)$ & -0.100 & $(0.060)$ \\
\hline & $\operatorname{CoVaR}\left(\mathrm{f} \mid \mathrm{d}=0.05, \mathrm{~d}^{*}\right)$ & -0.063 & $(0.036)$ & -0.056 & $(0.030)$ & -0.089 & $(0.046)$ \\
\hline & $\operatorname{CoVaR}\left(\mathrm{f} \mid \mathrm{d}, \mathrm{d}^{*}=0.05\right)$ & -0.058 & $(0.048)$ & -0.040 & $(0.023)$ & -0.124 & $(0.058)$ \\
\hline & $\operatorname{CoVaR}\left(\mathrm{f} \mid \mathrm{d}=0.05, \mathrm{~d}^{*}=0.05\right)$ & -0.066 & $(0.045)$ & -0.051 & $(0.030)$ & -0.124 & $(0.046)$ \\
\hline \multirow[t]{5}{*}{ Finland } & $\operatorname{VaR}(\mathrm{f})$ & -0.065 & $(0.020)$ & -0.066 & $(0.021)$ & -0.060 & $(0.018)$ \\
\hline & $\mathrm{CoVaR}(\mathrm{f} \mid \mathrm{d})$ & -0.047 & $(0.015)$ & -0.048 & $(0.015)$ & -0.044 & $(0.013)$ \\
\hline & $\operatorname{CoVaR}\left(\mathrm{f} \mid \mathrm{d}=0.05, \mathrm{~d}^{*}\right)$ & -0.047 & $(0.015)$ & -0.048 & $(0.015)$ & -0.043 & $(0.014)$ \\
\hline & $\operatorname{CoVaR}\left(\mathrm{f} \mid \mathrm{d}, \mathrm{d}^{*}=0.05\right)$ & -0.048 & $(0.017)$ & -0.048 & $(0.016)$ & -0.048 & $(0.019)$ \\
\hline & $\operatorname{CoVaR}\left(\mathrm{f} \mid \mathrm{d}=0.05, \mathrm{~d}^{*}=0.05\right)$ & -0.052 & $(0.017)$ & -0.052 & $(0.016)$ & -0.049 & $(0.019)$ \\
\hline \multirow[t]{5}{*}{ France } & $\operatorname{VaR}(\mathrm{f})$ & -0.078 & $(0.044)$ & -0.073 & $(0.045)$ & -0.095 & $(0.035)$ \\
\hline & $\operatorname{CoVaR}(\mathrm{f} \mid \mathrm{d})$ & -0.055 & $(0.031)$ & -0.052 & $(0.032)$ & -0.069 & $(0.025)$ \\
\hline & $\operatorname{CoVaR}\left(\mathrm{f} \mid \mathrm{d}=0.05, \mathrm{~d}^{*}\right)$ & -0.058 & $(0.035)$ & -0.055 & $(0.036)$ & -0.072 & $(0.027)$ \\
\hline & $\operatorname{CoVaR}\left(\mathrm{f} \mid \mathrm{d}, \mathrm{d}^{*}=0.05\right)$ & -0.057 & $(0.042)$ & -0.051 & $(0.040)$ & -0.081 & $(0.039)$ \\
\hline & $\operatorname{CoVaR}\left(\mathrm{f} \mid \mathrm{d}=0.05, \mathrm{~d}^{*}=0.05\right)$ & -0.062 & $(0.042)$ & -0.057 & $(0.042)$ & -0.083 & $(0.039)$ \\
\hline \multirow[t]{5}{*}{ Germany } & $\operatorname{VaR}(\mathrm{f})$ & -0.071 & $(0.036)$ & -0.071 & $(0.038)$ & -0.071 & $(0.023)$ \\
\hline & $\operatorname{CoVaR}(\mathrm{f} \mid \mathrm{d})$ & -0.042 & $(0.021)$ & -0.042 & $(0.023)$ & -0.043 & $(0.014)$ \\
\hline & $\operatorname{CoVaR}\left(\mathrm{f} \mid \mathrm{d}=0.05, \mathrm{~d}^{*}\right)$ & -0.031 & $(0.016)$ & -0.032 & $(0.017)$ & -0.027 & $(0.009)$ \\
\hline & $\operatorname{CoVaR}\left(\mathrm{f} \mid \mathrm{d}, \mathrm{d}^{*}=0.05\right)$ & -0.015 & $(0.023)$ & -0.013 & $(0.023)$ & -0.024 & $(0.019)$ \\
\hline & $\operatorname{CoVaR}\left(\mathrm{f} \mid \mathrm{d}=0.05, \mathrm{~d}^{*}=0.05\right)$ & -0.040 & $(0.027)$ & -0.041 & $(0.029)$ & -0.036 & $(0.018)$ \\
\hline \multirow[t]{2}{*}{ Greece } & $\operatorname{VaR}(\mathrm{f})$ & -0.091 & $(0.046)$ & -0.072 & $(0.028)$ & -0.164 & $(0.024)$ \\
\hline & CoVaR (f|d) & -0.105 & $(0.080)$ & -0.068 & $(0.033)$ & -0.246 & $(0.043)$ \\
\hline \multirow[t]{5}{*}{ Italy } & $\operatorname{VaR}(\mathrm{f})$ & -0.069 & $(0.037)$ & -0.060 & $(0.035)$ & -0.103 & $(0.023)$ \\
\hline & $\operatorname{CoVaR}(\mathrm{f} \mid \mathrm{d})$ & -0.075 & $(0.060)$ & -0.053 & $(0.035)$ & -0.163 & $(0.054)$ \\
\hline & $\operatorname{CoVaR}\left(\mathrm{f} \mid \mathrm{d}=0.05, \mathrm{~d}^{*}\right)$ & -0.070 & $(0.045)$ & -0.056 & $(0.031)$ & -0.120 & $(0.053)$ \\
\hline & $\operatorname{CoVaR}\left(\mathrm{f} \mid \mathrm{d}, \mathrm{d}^{*}=0.05\right)$ & -0.070 & $(0.065)$ & -0.043 & $(0.024)$ & -0.173 & $(0.071)$ \\
\hline & $\operatorname{CoVaR}\left(\mathrm{f} \mid \mathrm{d}=0.05, \mathrm{~d}^{*}=0.05\right)$ & -0.067 & $(0.056)$ & -0.044 & $(0.028)$ & -0.154 & $(0.050)$ \\
\hline \multirow[t]{5}{*}{ Netherlands } & $\operatorname{VaR}(\mathrm{f})$ & -0.091 & $(0.052)$ & -0.085 & $(0.052)$ & -0.117 & $(0.042)$ \\
\hline & CoVaR (f|d) & -0.049 & $(0.033)$ & -0.047 & $(0.034)$ & -0.059 & $(0.029)$ \\
\hline & $\operatorname{CoVaR}\left(\mathrm{f} \mid \mathrm{d}=0.05, \mathrm{~d}^{*}\right)$ & -0.070 & $(0.045)$ & -0.064 & $(0.044)$ & -0.091 & $(0.042)$ \\
\hline & $\operatorname{CoVaR}\left(\mathrm{f} \mid \mathrm{d}, \mathrm{d}^{*}=0.05\right)$ & -0.066 & $(0.056)$ & -0.050 & $(0.046)$ & -0.128 & $(0.050)$ \\
\hline & $\operatorname{CoVaR}\left(\mathrm{f} \mid \mathrm{d}=0.05, \mathrm{~d}^{*}=0.05\right)$ & -0.070 & $(0.056)$ & -0.055 & $(0.046)$ & -0.130 & $(0.051)$ \\
\hline
\end{tabular}


Table 4.6 (Continued)

\begin{tabular}{|c|c|c|c|c|c|c|c|}
\hline \multirow[t]{5}{*}{ Portugal } & $\operatorname{VaR}(\mathrm{f})$ & -0.064 & $(0.044)$ & -0.050 & $(0.031)$ & -0.121 & $(0.042)$ \\
\hline & $\mathrm{CoVaR}(\mathrm{f} \mid \mathrm{d})$ & -0.075 & $(0.067)$ & -0.048 & $(0.029)$ & -0.180 & $(0.067)$ \\
\hline & $\mathrm{CoVaR}\left(\mathrm{f} \mid \mathrm{d}=0.05, \mathrm{~d}^{*}\right)$ & -0.076 & $(0.055)$ & -0.059 & $(0.040)$ & -0.139 & $(0.058)$ \\
\hline & $\mathrm{CoVaR}\left(\mathrm{f} \mid \mathrm{d}, \mathrm{d}^{*}=0.05\right)$ & -0.101 & $(0.078)$ & -0.071 & $(0.043)$ & -0.216 & $(0.074)$ \\
\hline & CoVaR $\left(\mathrm{f} \mid \mathrm{d}=0.05, \mathrm{~d}^{*}=0.05\right)$ & -0.078 & $(0.066)$ & -0.052 & $(0.033)$ & -0.179 & $(0.067)$ \\
\hline \multirow[t]{5}{*}{ Spain } & $\operatorname{VaR}(\mathrm{f})$ & -0.073 & $(0.038)$ & -0.066 & $(0.038)$ & -0.099 & $(0.021)$ \\
\hline & CoVaR (f|d) & -0.067 & $(0.043)$ & -0.057 & $(0.038)$ & -0.107 & $(0.037)$ \\
\hline & $\operatorname{CoVaR}\left(\mathrm{f} \mid \mathrm{d}=0.05, \mathrm{~d}^{*}\right)$ & -0.066 & $(0.035)$ & -0.057 & $(0.030)$ & -0.100 & $(0.029)$ \\
\hline & $\operatorname{CoVaR}\left(\mathrm{f} \mid \mathrm{d}, \mathrm{d}^{*}=0.05\right)$ & -0.065 & $(0.045)$ & -0.052 & $(0.037)$ & -0.113 & $(0.043)$ \\
\hline & $\operatorname{CoVaR}\left(\mathrm{f} \mid \mathrm{d}=0.05, \mathrm{~d}^{*}=0.05\right)$ & -0.068 & $(0.043)$ & -0.057 & $(0.037)$ & -0.111 & $(0.037)$ \\
\hline
\end{tabular}

Notes. The table reports descriptive VaR and CoVaR statistics at the $95 \%$ confidence level for domestic financial sectors and sovereign debt markets for selected countries in the overall sample and in the pre- and post-onset crisis periods using the best copula fit. $\mathrm{CoVaR}(\mathrm{f} \mid \mathrm{d})$ denotes the $\mathrm{CoVaR}$ of the financial system conditional on the fact that the sovereign debt market is in distress; $\operatorname{CoVaR}\left(\mathrm{f} \mid \mathrm{d}=0.05, \mathrm{~d}^{*}\right)$ denotes the same but takes into account the effect of the sovereign Greek debt market situation. $\operatorname{CoVaR}\left(\mathrm{f} \mid \mathrm{d}, \mathrm{d}^{*}=0.05\right)$ denotes the CoVaR of the financial system given the situation in the domestic debt market and conditional on the fact that the sovereign Greek debt market is in distress; finally, $\operatorname{CoVaR}\left(\mathrm{f} \mid \mathrm{d}=0.05, \mathrm{~d}^{*}=0.05\right)$ denotes the $\mathrm{CoVaR}$ of the financial system conditional on the fact that the domestic and sovereign Greek debt markets are in distress. Values reported are mean and standard deviations (SD).

Figure 4.9 depicts CoVaR dynamics throughout the sample period for the vine-copula model, whose CoVaR was computed under three scenarios: (1) the $\mathrm{CoVaR}$ of the domestic financial system given domestic sovereign distress (represented by $\mathrm{d}=0.05$ ) and the current situation in the Greek debt market (not necessarily in distress); (2) the CoVaR of the domestic financial system given the domestic sovereign debt market situation (not necessarily in distress) and the sovereign Greek debt market in distress (represented by $\mathrm{d}^{*}=0.05$ ); and (3) the $\mathrm{CoVaR}$ of the domestic financial system when domestic and Greek sovereign debt markets are both in distress (represented by $\mathrm{d}=0.05$ and $\mathrm{d}^{*}=0.05$, respectively). Average CoVaR data for the three scenarios for the entire sample and in the preand post-onset European sovereign debt crisis periods are provided in the last three rows for each country in Table 4.6. Our results indicate that the systemic effect of a potential Greek sovereign debt default on the financial system of Austria, Finland, France and Germany was negligible, as CoVaR computed under scenarios (1) and (2) were no different from the CoVaR obtained without considering the impact of Greek sovereign debt. This evidence is consistent with the fact that the Greek debt market decoupled from the debt markets and financial systems of those core countries (as shown by our previous copula results). Note also that the weight of the Greek sovereign debt in the bank portfolios of those countries was relatively low 
(Battistini et al., 2014). However, for Belgium and Netherlands the systemic impact of the Greek sovereign debt crisis was different: CoVaR figures under scenarios (2) and (3) were lower than for the CoVaR figures obtained without considering the impact of Greek sovereign debt. For Belgium, this evidence was a consequence of the change in the dependence relationship between Greek sovereign debt, domestic debt and financial systems; for the Netherlands, this was also the consequence of Dutch investments in sovereign Greek debt (see Blundell-Wignall and Slovik, 2010). Regarding the peripheral countries, the systemic impact of sovereign Greek debt distress had a limited impact in Spain, given that the CoVaR under scenarios (2) and (3) did not differ greatly from the CoVaR obtained when the impact of Greek sovereign debt was not considered. For Italy and Portugal, however, systemic impact was patent.

Figure 4.9: Results for $\operatorname{CoVaR}\left(\mathrm{f} \mid \mathrm{d}=0.05, \mathrm{~d}^{*}\right), \operatorname{CoVaR}\left(\mathrm{f} \mid \mathrm{d}, \mathrm{d}^{*}=0.05\right)$ and $\mathrm{CoVaR}$

$$
\left(\mathrm{f} \mid \mathrm{d}=0.05, \mathrm{~d}^{*}=0.05\right) \text {. }
$$

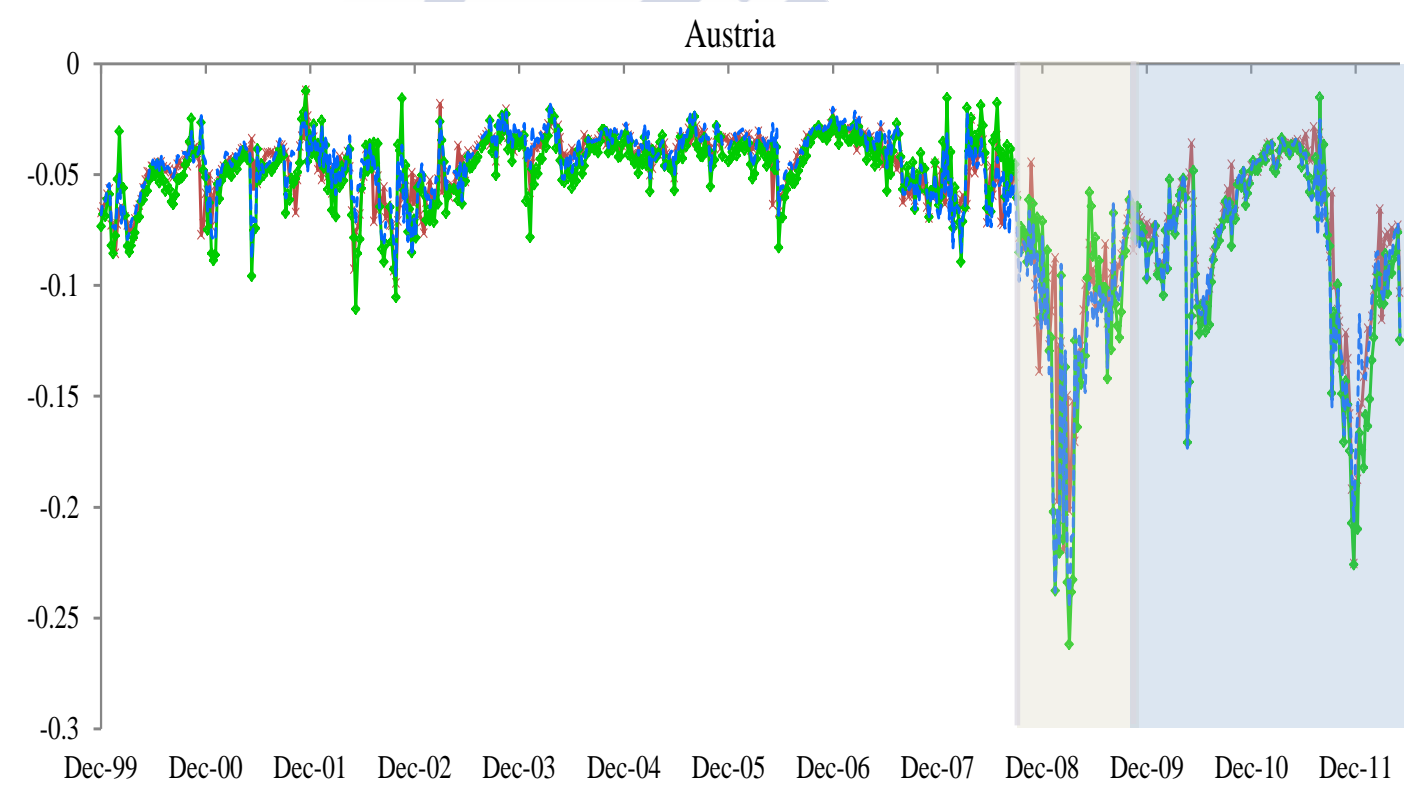

$\longrightarrow \operatorname{CoVaR}\left(\mathrm{f} \mid \mathrm{d}=0.05, \mathrm{~d}^{*}\right) \quad \longrightarrow \operatorname{CoVaR}\left(\mathrm{f} \mid \mathrm{d}, \mathrm{d}^{*}=0.05\right) \quad-\cdots-\cdots \operatorname{CoVaR}\left(\mathrm{f} \mid \mathrm{d}=0.05, \mathrm{~d}^{*}=0.05\right)$ 


\section{Figure 4.9: (Continued)}
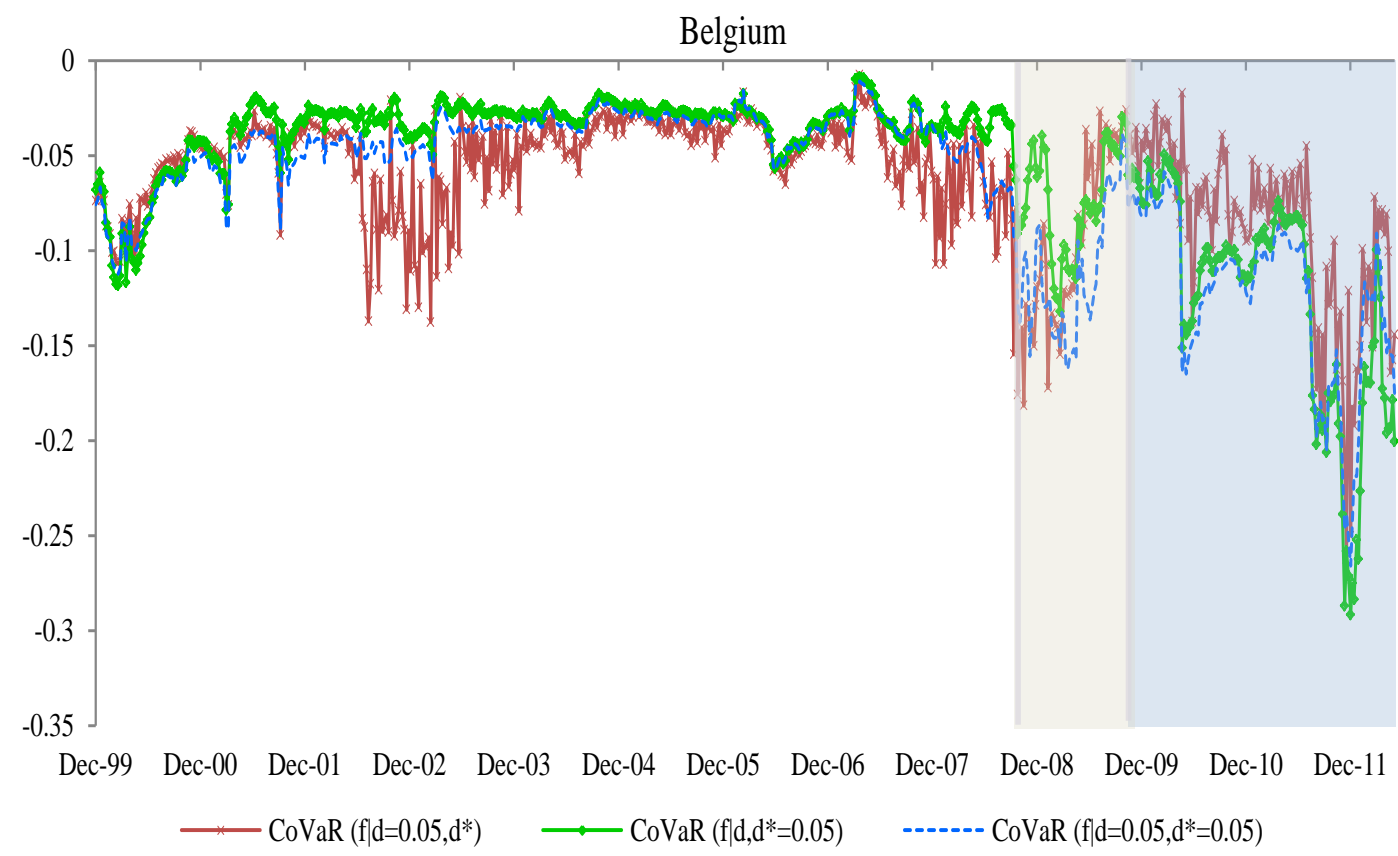

$\operatorname{CoVaR}\left(\mathrm{f} \mid \mathrm{d}=0.05, \mathrm{~d}^{*}=0.05\right)$

\section{Finland}

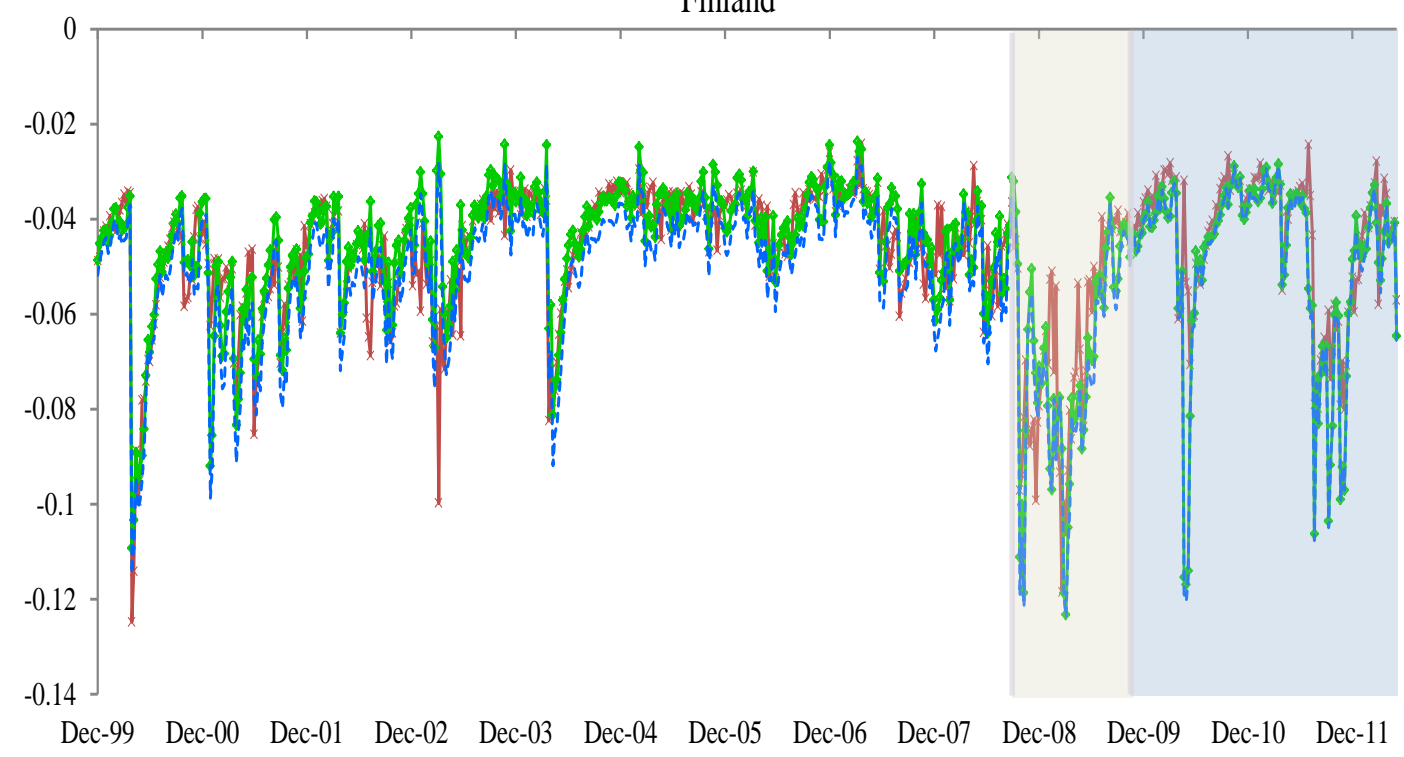

$\longrightarrow \operatorname{CoVaR}\left(\mathrm{f} \mid \mathrm{d}=0.05, \mathrm{~d}^{*}\right)$

$\mathrm{CoVaR}\left(\mathrm{f} \mid \mathrm{d}, \mathrm{d}^{*}=0.05\right)$

$\operatorname{CoVaR}\left(\mathrm{f} \mid \mathrm{d}=0.05, \mathrm{~d}^{*}=0.05\right)$ 
Figure 4.9: (Continued)
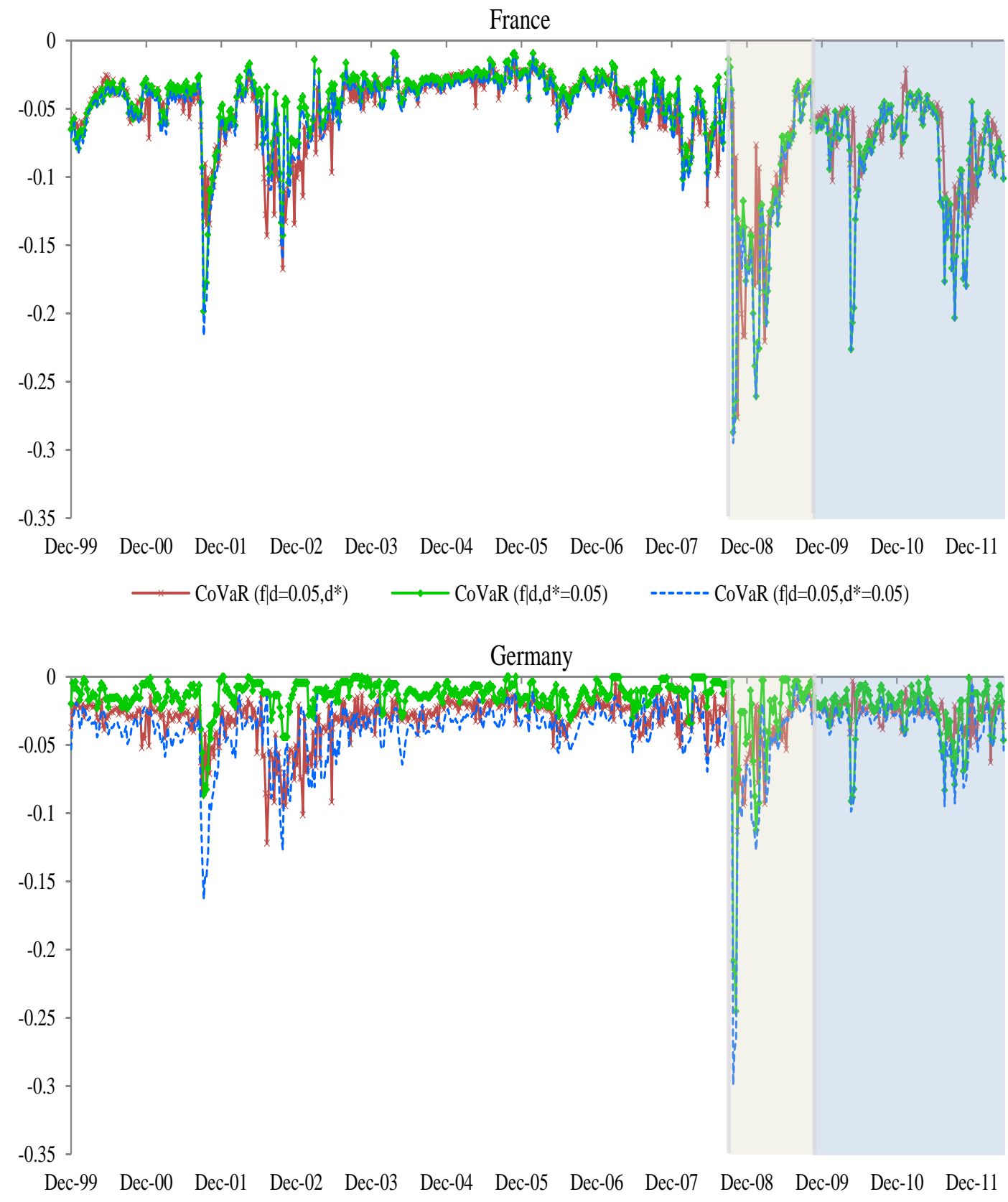

$\longrightarrow \operatorname{CoVaR}\left(\mathrm{f} \mid \mathrm{d}=0.05, \mathrm{~d}^{*}\right) \quad \longrightarrow \operatorname{CoVaR}\left(\mathrm{f} \mid \mathrm{d}, \mathrm{d}^{*}=0.05\right) \quad----\cdot \operatorname{CoVaR}\left(\mathrm{f} \mid \mathrm{d}=0.05, \mathrm{~d}^{*}=0.05\right)$ 


\section{Figure 4.9: (Continued)}

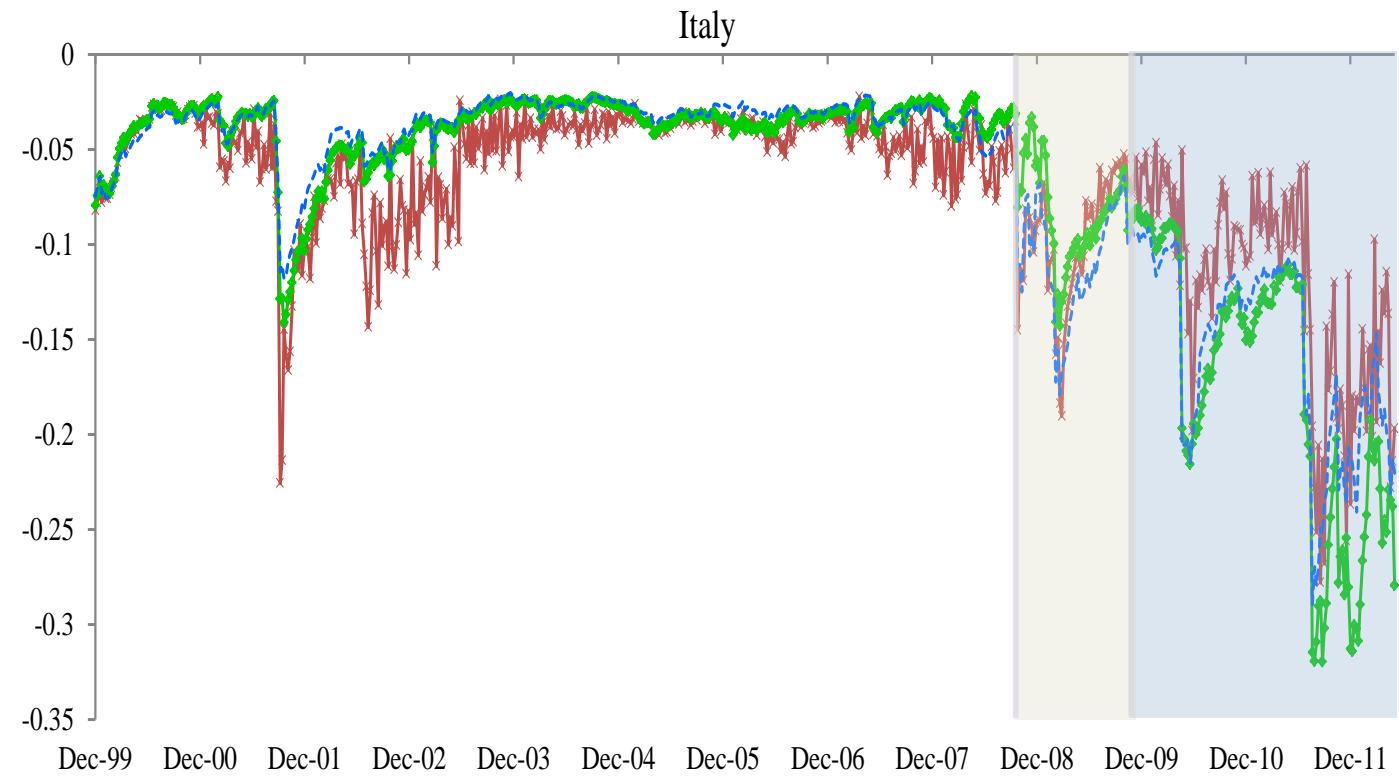

$\longrightarrow \operatorname{CoVaR}\left(\mathrm{f} \mid \mathrm{d}=0.05, \mathrm{~d}^{*}\right) \quad \longrightarrow \operatorname{CoVaR}\left(\mathrm{f} \mid \mathrm{d}, \mathrm{d}^{*}=0.05\right) \quad \cdots \cdots \operatorname{CoVaR}\left(\mathrm{f} \mid \mathrm{d}=0.05, \mathrm{~d}^{*}=0.05\right)$

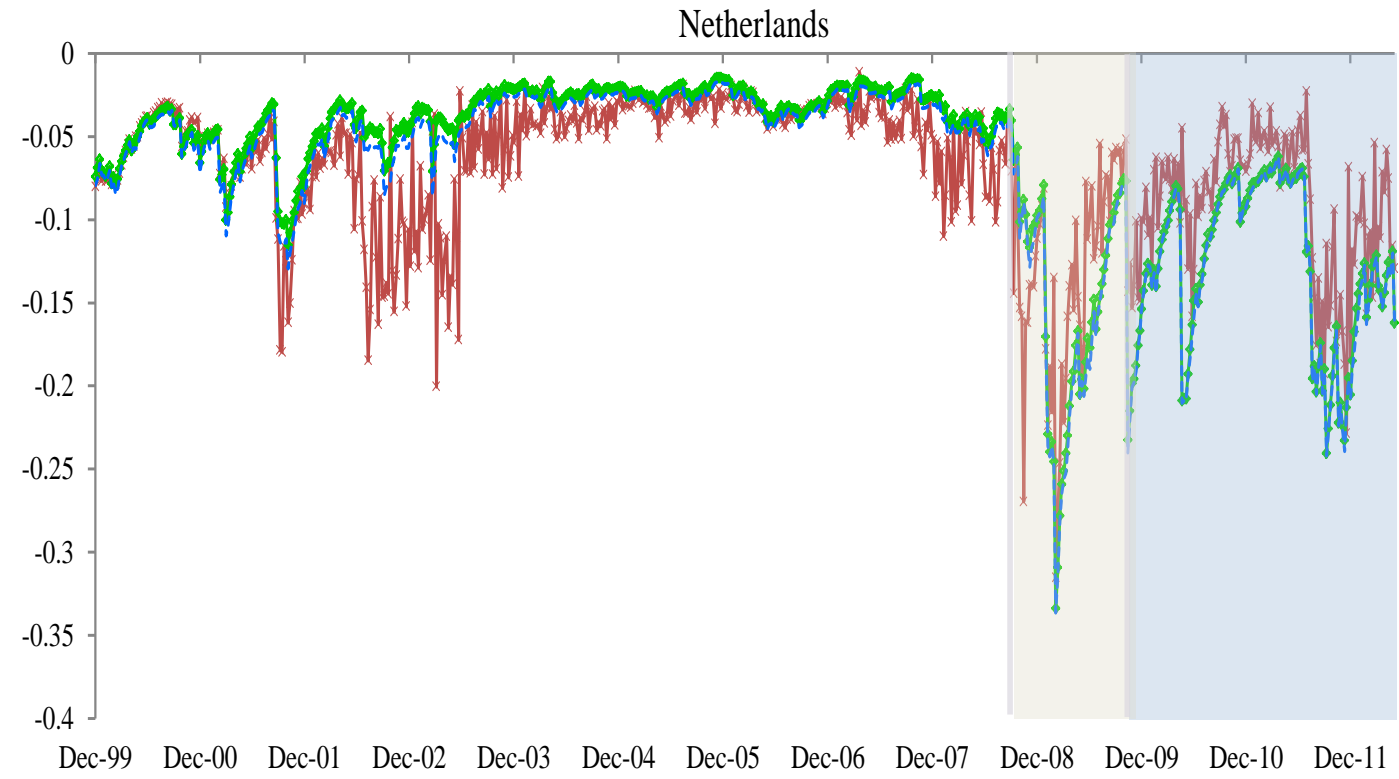

$\longrightarrow \operatorname{CoVaR}\left(\mathrm{f} \mid \mathrm{d}=0.05, \mathrm{~d}^{*}\right) \quad \longrightarrow \operatorname{CoVaR}\left(\mathrm{f} \mid \mathrm{d}, \mathrm{d}^{*}=0.05\right) \quad \cdots \operatorname{CoVaR}\left(\mathrm{f} \mid \mathrm{d}=0.05, \mathrm{~d}^{*}=0.05\right)$ 
Figure 4.9: (Continued)

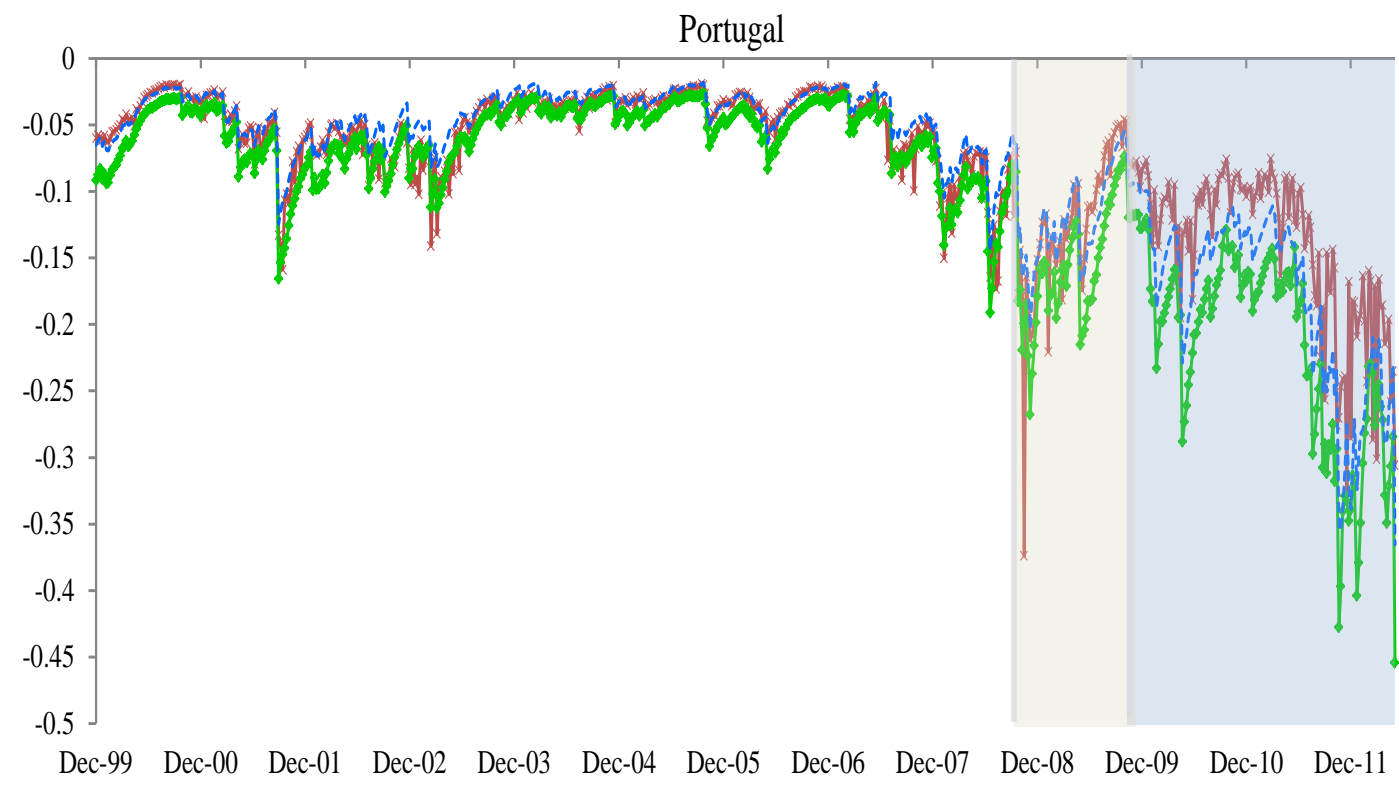

$\longrightarrow \operatorname{CoVaR}\left(\mathrm{f} \mid \mathrm{d}=0.05, \mathrm{~d}^{*}\right) \quad \longrightarrow \operatorname{CoVaR}\left(\mathrm{f} \mid \mathrm{d}, \mathrm{d}^{*}=0.05\right) \quad \cdots \cdots \operatorname{CoVaR}\left(\mathrm{f} \mid \mathrm{d}=0.05, \mathrm{~d}^{*}=0.05\right)$

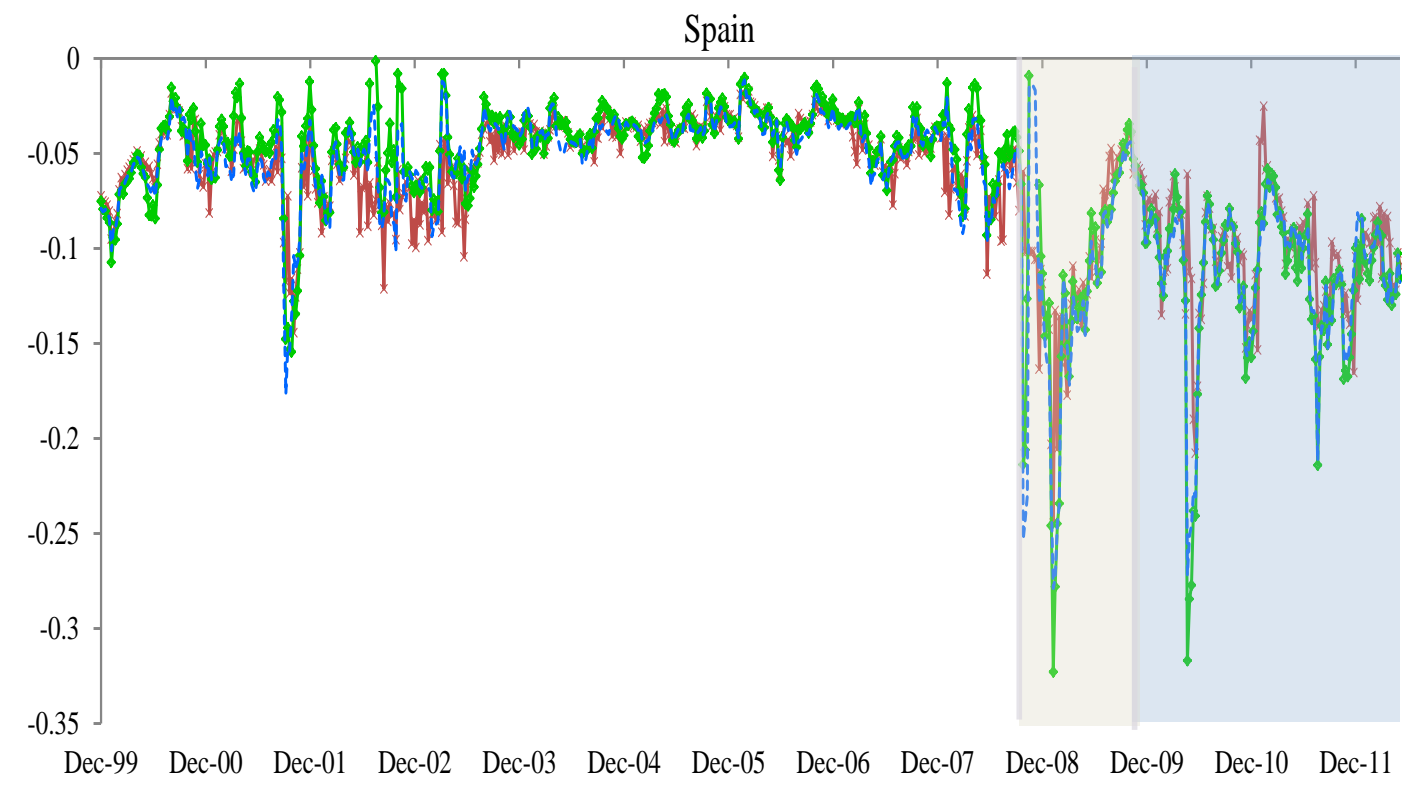

$\longrightarrow \operatorname{CoVaR}\left(\mathrm{f} \mid \mathrm{d}=0.05, \mathrm{~d}^{*}\right) \quad \longrightarrow \operatorname{CoVaR}\left(\mathrm{f} \mid \mathrm{d}, \mathrm{d}^{*}=0.05\right) \quad \cdots-\cdots \operatorname{CoVaR}\left(\mathrm{f} \mid \mathrm{d}=0.05, \mathrm{~d}^{*}=0.05\right)$ 
Overall, our CoVaR results indicate that: (1) domestic sovereign debt had a systemic impact on domestic financial systems across European countries that was positive in the sense of increasing financial system VaR; (2) this systemic effect changed with the onset of the European debt crisis in peripheral countries like Greece, Italy and Portugal, where the systemic impact of sovereign debt increased, thereby reducing financial system CoVaR; (3) the systemic impact of a potential Greek debt default was mainly limited to Belgium, Italy, the Netherlands and Portugal; and finally, (4) for the remaining countries this event does not add much value with respect to the CoVaR figures obtained without considering the impact of the Greek sovereign debt.

\subsubsection{Statistical test}

Empirical evidence reported in Section 4.3 describes systemic risk behaviour before and after crisis onset with no testing of statistical significance. Below we use statistical significance testing to draw robust conclusions on sovereign debt systemic risk for financial systems. We compared cumulative distribution for $\mathrm{VaR}$ and/or CoVaR data using the Kolmogorov-Smirnov (KS) bootstrapping test as proposed by Abadie (2002) and applied by Bernal et al. (2014) to compare CoVaR figures. This test measures the difference between two cumulative quantile functions relying on the empirical distribution function and without considering any underlying distribution function. It is defined as:

$$
\mathrm{KS}_{\mathrm{mn}}=\left(\frac{\mathrm{mn}}{\mathrm{m}+\mathrm{n}}\right)^{\frac{1}{2}} \sup _{\mathrm{x}}\left|\mathrm{F}_{\mathrm{m}}(\mathrm{x})-\mathrm{G}_{\mathrm{n}}(\mathrm{x})\right|,
$$

where $F_{m}(x)$ and $G_{n}(x)$ are the cumulative $V a R$ or CoVaR distribution functions, respectively, and $n$ and $m$ are the size of the two samples. With this statistic we tested four hypotheses, both before and after crisis onset:

- Hypothesis 1: $\mathrm{H}_{0}: \operatorname{CoVaR}(\mathrm{f} \mid \mathrm{d})>\operatorname{VaR}(\mathrm{f})$

- Hypothesis $2: \mathrm{H}_{0}: \operatorname{CoVaR}(\mathrm{f} \mid \mathrm{d})>\operatorname{CoVaR}\left(\mathrm{f} \mid \mathrm{d}=0.05, \mathrm{~d}^{*}\right)$

- Hypothesis 3: $\mathrm{H}_{0}: \operatorname{CoVaR}(\mathrm{f} \mid \mathrm{d})>\operatorname{CoVaR}\left(\mathrm{f} \mid \mathrm{d}, \mathrm{d}^{*}=0.05\right)$

- Hypothesis 4: $\mathrm{H}_{0}: \operatorname{CoVaR}(\mathrm{f} \mid \mathrm{d})>\operatorname{CoVaR}\left(\mathrm{f} \mid \mathrm{d}=0.05, \mathrm{~d}^{*}=0.05\right)$

The first hypothesis examines whether sovereign debt contributes to downside risk in the financial system, whereas the remaining hypotheses examine whether systemic risk in one country is affected by the Greek debt market under normal or exceptional circumstances (denominated scenarios (1)-(3) above). Table 4.7 reports results for the KS statistic and the associated bootstrap p-values under the null 
A Vine-copula CoVaR approach to systemic sovereign debt risk for the financial...

hypothesis, yielding statistical evidence that is fully consistent with the empirical evidence described in Section 4.3.

Table 4.7: Significant test for differences in risk measures.

\begin{tabular}{|c|c|c|c|c|c|c|c|c|c|}
\hline & & \multicolumn{2}{|c|}{ Hypothesis 1} & \multicolumn{2}{|c|}{ Hypothesis 2} & \multicolumn{2}{|c|}{ Hypothesis 3} & \multicolumn{2}{|c|}{ Hypothesis 4} \\
\hline & & Stat & p-value & Stat & p-value & Stat & p-value & Stat & $\mathrm{p}$-value \\
\hline \multirow[t]{2}{*}{ Austria } & Before crisis onset & 0.000 & 0.999 & 0.033 & 0.567 & 0.024 & 0.724 & 0.033 & 0.544 \\
\hline & After crisis onset & 0.000 & 0.999 & 0.098 & 0.271 & 0.008 & 0.988 & 0.008 & 0.990 \\
\hline \multirow[t]{2}{*}{ Belgium } & Before crisis onset & 0.000 & 0.999 & 0.010 & 0.950 & 0.285 & 0.000 & 0.031 & 0.601 \\
\hline & After crisis onset & 0.256 & 0.000 & 0.158 & 0.032 & 0.000 & 0.999 & 0.023 & 0.925 \\
\hline \multirow[t]{2}{*}{ Finland } & Before crisis onset & 0.000 & 0.999 & 0.056 & 0.194 & 0.109 & 0.002 & 0.021 & 0.782 \\
\hline & After crisis onset & 0.000 & 0.999 & 0.105 & 0.219 & 0.030 & 0.873 & 0.030 & 0.874 \\
\hline \multirow[t]{2}{*}{ France } & Before crisis onset & 0.000 & 0.999 & 0.017 & 0.842 & 0.085 & 0.022 & 0.027 & 0.652 \\
\hline & After crisis onset & 0.000 & 0.999 & 0.030 & 0.878 & 0.023 & 0.923 & 0.015 & 0.964 \\
\hline \multirow[t]{2}{*}{ Germany } & Before crisis onset & 0.000 & 0.999 & 0.308 & 0.000 & 0.816 & 0.000 & 0.101 & 0.004 \\
\hline & After crisis onset & 0.000 & 0.999 & 0.752 & 0.000 & 0.722 & 0.000 & 0.526 & 0.000 \\
\hline \multirow[t]{2}{*}{ Greece } & Before crisis onset & 0.081 & 0.031 & & & & & & \\
\hline & After crisis onset & 0.895 & 0.000 & & & & & & \\
\hline \multirow[t]{2}{*}{ Italy } & Before crisis onset & 0.010 & 0.949 & 0.041 & 0.413 & 0.213 & 0.000 & 0.291 & 0.000 \\
\hline & After crisis onset & 0.617 & 0.000 & 0.466 & 0.000 & 0.150 & 0.046 & 0.248 & 0.000 \\
\hline \multirow[t]{2}{*}{ Netherlands } & Before crisis onset & 0.000 & 0.999 & 0.000 & 0.999 & 0.083 & 0.029 & 0.000 & 0.999 \\
\hline & After crisis onset & 0.008 & 0.988 & 0.000 & 0.999 & 0.000 & 0.999 & 0.000 & 0.999 \\
\hline \multirow[t]{2}{*}{ Portugal } & Before crisis onset & 0.029 & 0.636 & 0.000 & 0.999 & 0.000 & 0.999 & 0.002 & 0.997 \\
\hline & After crisis onset & 0.504 & 0.000 & 0.406 & 0.000 & 0.000 & 0.999 & 0.113 & 0.179 \\
\hline \multirow[t]{2}{*}{ Spain } & Before crisis onset & 0.010 & 0.950 & 0.025 & 0.718 & 0.106 & 0.003 & 0.015 & 0.878 \\
\hline & After crisis onset & 0.128 & 0.110 & 0.113 & 0.173 & 0.045 & 0.751 & 0.023 & 0.924 \\
\hline
\end{tabular}

Note. The bootstrap Kolmogorov-Smirnov tests whether the values of different risk measures follow (or not) the same cumulative distribution function (CDFs) in the pre- and post-onset crisis periods. The null hypotheses are considered as follows:

Hypothesis $1=\mathrm{H}_{0}: \operatorname{CoVaR}(\mathrm{f} \mid \mathrm{d})>\operatorname{VaR}(\mathrm{f})$

Hypothesis $2=\mathrm{H}_{0}: \operatorname{CoVaR}(\mathrm{f} \mid \mathrm{d})>\operatorname{CoVaR}\left(\mathrm{f} \mid \mathrm{d}=0.05, \mathrm{~d}^{*}\right)$

Hypothesis $3=\mathrm{H}_{0}$ : $\operatorname{CoVaR}(\mathrm{f} \mid \mathrm{d})>\operatorname{CoVaR}\left(\mathrm{f} \mid \mathrm{d}, \mathrm{d}^{*}=0.05\right)$

Hypothesis $4=\mathrm{H}_{0}$ : $\operatorname{CoVaR}(\mathrm{f} \mid \mathrm{d})>\operatorname{CoVaR}\left(\mathrm{f} \mid \mathrm{d}=0.05, \mathrm{~d}^{*}=0.05\right)$.

\subsection{Conclusions}

We have provided empirical evidence, for the periods before and after the onset of the recent financial and debt crises, of (1) the systemic impact of domestic sovereign debt distress on domestic financial systems in European countries and (2) the potential systemic impact of a distressed Greek debt market on the financial systems of other European countries. We measured systemic risk using the CoVaR measure, 
as proposed by Adrian and Brunnermeier (2011) and generalized by Girardi and Ergün (2013). CoVaR measures VaR for a financial system conditional on the fact that the debt market is in distress. We computed CoVaR data using both bivariate and vine-copula models, given that copulas can flexibly account for dependence, most especially for tail dependence, which is crucial to determining CoVaR data. To estimate CoVaR, we adopted (1) a two-step procedure that accounted for the impact of domestic debt distress on domestic financial systems, and (2) a three-step procedure that-taking into account the link between domestic financial and sovereign debt markets - accounted for the systemic impact of a potential Greek default on the financial systems of other European countries.

Using a sample of MSCI financial and sovereign bond benchmark indices for six eurozone core countries (Austria, Belgium, Finland, France, Germany and the Netherlands) and four peripheral countries (Italy, Greece, Portugal and Spain) for the period 2000 to 2012, we estimated copula and vine-copula models - in order to characterize the dependence structure between financial and sovereign debt markets - and then computed CoVaR figures. Our evidence indicates that there were substantial differences in the systemic impact of sovereign debt in the periods before and after the onset of the European debt crisis. In the pre-onset period sovereign debt was observed to have a positive systemic risk effect in reducing financial system VaR. This impact can be explained in terms of the diversification effect of sovereign debt on bank portfolios, with even Greek sovereign debt playing a diversification role across financial systems in the eurozone, except in Portugal. However, in the post-onset crisis period the picture was quite different, with domestic sovereign debt having a negative systemic impact on domestic financial systems as CoVaR fell. This evidence was found for all the peripheral countries; as for the core countries, sovereign debt continued to play a diversification role, having a positive impact on CoVaR. This positive impact can be explained by the fact that the negative impact of the sovereign debt crisis was not fully transmitted to the core countries. Regarding the systemic impact of Greek sovereign debt distress on the financial systems of other countries, we found all financial systems in Europe to be affected, but to differing degrees. For core countries, after the onset of the debt crisis, Greek sovereign debt continued to play a diversification effect, although this effect was less intense and more varied. In the four peripheral countries, Belgium and the Netherlands, the systemic effects of Greek sovereign debt distress exacerbated financial system risk. 


\section{References}

Aas, K., Berg, D., 2009. Models for construction of multivariate dependence: a comparison study. European Journal of Finance 15, 639-659.

Aas, K., Czado, C., Frigessi, A., Bakken, H., 2009. Pair-copula constructions of multiple dependence. Insurance: Mathematics and Economics 44, 182-198.

Abadie, A., 2002. Bootstrap tests for distributional treatment effects in instrumental variables models. Journal of American Statistical Association 97(457), 284-292.

Acharya V., 2009. A theory of systemic risk and design of prudential bank regulation. Journal of Financial Stability n. 5, 224-255.

Acharya, V., Pedersen, L.H., Philippon, T., Richardson, M., 2010. Measuring Systemic Risk. Working paper, New York University.

Adrian, T., Brunnermeier, M.K., 2011. CoVaR. NBER working paper series, w17454.

Alfaro, R., and Drehmann M., 2009. Macro stress tests and crises: what can we learn?. BIS Quarterly Review, pp. 29-41.

Allen, F., Babus, A., Carletti, E., 2010. Financial connections and systemic risk. NBER Working Paper No. 16177.

Allen, L., Bali, T.G., Tang, Y., 2010. Does systemic risk in the financial sector predict future economic downturns? Working Paper, SSRN.

Alter, A., Beyer, A., 2012. The dynamics of spillover effects during the European sovereign debt turmoil. CFS Working Paper 13.

Alter, A., Schuler, Y., 2012. Credit spread interdependencies of European states and banks during the financial crisis. Journal of Banking and Finance 36(12), 34443468 .

Arezki, R., Candelon, B., Sy, A., 2011. Sovereign Rating News and Financial Markets Spillovers: Evidence from European Debt Crisis. IMF Staff Papers Working Paper $11 / 68$.

Battistini, N., Pagano, M., Simonelli, S., 2014. Systemic risk, sovereign yields and bank exposures in the euro crisis. Economic Policy, 203-251.

Beetsma, R., Giuliodori, M., de Jong, F., Widijanto, D., 2013. Spread the news: the impact of news on the European sovereign bond markets during the crisis. Journal of International Money and Finance 34, 83-101. 
Bernal. O., Gnabo, J-Y., Guilmin, G., 2014. Assessing the contribution of banks, insurance and other financial services to systemic risk. Journal of Banking and Finance, http://dx.doi.org/10.1016/j.jbankfin.2014.05.030

Bhanot, K., Burns, N., Hunter, D., Williams, M., 2014. News spillovers from the Greek debt crisis: Impact on the Eurozone financial sector. Journal of Banking \& Finance $38,51-63$.

Monica B., Getmansky M., Lo A.W., Pelizzon L., 2010. Measuring systemic risk in the finance and insurance sectors. MIT.

Billio, M., Getmansky, M., Lo, A.W., Pelizzon, L., 2012. Econometric measures of systemic risk in the finance and insurance sectors. Journal of Financial Economics $104,535-559$.

BIS, 2011. The impact of sovereign credit risk on bank funding conditions. Committee on the global financial system papers, 43.

Bisias, D., Flood, M.D., Lo, A.W., Valavanis, S., 2012. A survey of systemic risk analytics. U.S. Department of Treasury, Office of Financial Research No. 0001.

Blundell-Wignall, A. and P. Slovik (2010), "The EU Stress Test and Sovereign Debt Exposures", OECD Working Papers on Finance, Insurance and Private Pensions, No. 4, OECD Financial Affairs Division, www.oecd.org/daf/fin.

Borgy, V., Laubach, T., Mesonnier, J.-S., Renne, J.-P., 2011. Fiscal sustainability, default risk and euro-area government spreads. Available at SSRN: http://ssrn.com/abstract=1943516.

Breymann, W., Dias, A., Embrechts, P., 2003. Dependence structures for multivariate high-frequency data in finance. Quantitative Finance 3, pp. 1-16.

Brownlees, C., Engle, R., 2012. Volatility, Correlation and Tails for Systemic Risk Measurement. Working paper, New York University.

Brunnermeier, M., Garicano, L., Lane, P.R., Pagano, M., Reis, R., Santos, T., Van Nieuwerburg, S., Vayanos, D., 2011. European safe bonds: ESBies. Euronomics.com.

Caporin, M., Pelizzon, L., Ravazzolo, F., Rigobon, R., 2013. Measuring sovereign contagion in Europe. NBER Working Paper 18741.

Chan, N., Getmansky, M., Haas, S. M., Lo, A.W., 2006a. Do Hedge Funds Increase Systemic Risk?. Federal Reserve Bank of Atlanta Economic Review, 91(4), pp. 4980 . 
Chan, N., Getmansky, M., Haas, S. M., Lo, A.W., 2006b. Systemic risk and hedge funds. The Risks of Financial Institutions, ed. by Carey M., Stulz R.. University of Chicago Press, Chicago, IL, pp. 235-330.

Chan-Lau, J., Espinosa, M., Sole, J., 2009. On the use of network analysis to assess systemic financial linkages. IMF working paper, International Monetary Fund.

Cherubini, U., Luciano, E., Vecchiato, W., 2004. Copula methods in finance. John Willey \& Sons: Chichester, UK.

Chollete, L., Heinen, A., Valdesogo, A., 2009. Modeling international financial returns with a multivariate regime-switching copula. Journal of Financial Econometrics 7, 437-480.

De Bandt, O., Hartmann, P., 2000. Systemic risk: A survey. ECB Working Paper Series, n. 35 .

De Bruyckere, V., Gerhardt, M., Schepens, G., Vennet, R.V., 2013. Bank/sovereign risk spillovers in the European debt crisis. Journal of Banking \& Finance 37, 4793-4809.

De Jonghe, O., 2010. Back to the basics in banking? A micro-analysis of banking system stability. Journal of Financial Intermediation 19, 387-417.

De Nicolò, G., Lucchetta, M., 2010. Systemic Risks and the Macroeconomy. IMF Working Paper No. 10/29, International Monetary Fund.

De Santis, R., 2013, The Euro Area's Sovereign Debt Crisis: Flight-to-liquidity and the spillover mechanism. Journal of Empirical Finance, forthcoming.

Diamond, D. W., Dybvig, P.H., 1983. Bank Runs, Deposit Insurance, and Liquidity. Journal of Political Economy, 91(3), 401-419.

Dickey, D.A., Fuller, W.A., 1979. Distribution of the estimators for autoregressive time series with a unit root. Journal of the American Statistical Society 75, 427-431.

ECB, 2009b. The concept of systemic risk. A special feature in Financial Stability Review.

Embrechts, P., Lindskog, F., McNeil, A., 2003. Modelling dependence with copulas and applications to risk management. In: Rachev, S. (Ed.), Handbook of Heavy Tailed Distributions in Finance. Elsevier, The Netherlands.

Engle, R.F., 2002. Dynamic Conditional Correlation - A Simple Class of Multivariate GARCH Models. Journal of Business and Economic Statistics, 20, 339-350.

Engle, R.F., Manganelli, S., 2004. Caviar: Conditional autoregressive value at risk by regression quantiles. Journal of Business and Economic Statistics 22, 367-381. 
Favero, C.A., 2013. Modelling and forecasting government bond spreads in the euro area: A GVAR model. Journal of Econometrics 177, 343-356.

Furfine, C., 2003. Interbank exposure: quantifying the risk of contagion. Journal of Money, Credit and Banking n. 35, pp. 111-128.

Giesecke, K., Kim, B., 2011. Systemic Risk: What Defaults Are Telling Us. Management Science, 57 (8), pp. 1387-1405.

Girardi, G., Ergün, A.T., 2013. Systemic risk measurement: Multivariate GARCH estimation of CoVaR. Journal of Banking and Finance 37, 3169-3180.

Glosten, L.R., Jaganathan, R., Runkle, D., 1993. On the relation between the expected value and the volatility of the normal excess return on stocks. Journal of Finance 48, 1779-1801.

Gravelle, T., Li, F., 2013. Measuring systemic importance of financial institutions: An extreme value theory approach. Journal of Banking and Finance 37, 2196-2209.

Gray, D.F.F., Jobst, A.A., 2010. New directions in financial sector and sovereign risk management. Journal of Investment Management 8, 23-38.

Halaj, G., Kok Sorensen, C., 2013. Assessing interbank contagion using simulated networks. Computational Management Science 10, 157-186.

Hansen, B., 1994. Autoregressive conditional density estimation. International Economic Review 35, 705-730.

Haugh, D., Ollivaud, P., Turner, D., 2009. What drives sovereign risk premiums? An analysis of recent evidence from the euro area. OECD Economics Department, Working paper no. 718.

Hobæk Haff, I., 2013. Parameter estimation for pair-copula constructions. Bernoulli 19 (2), 462-491.

Huang, X., Zhou, H., Zhu, H., 2009. A framework for assessing the systemic risk of major financial institutions. Journal of Banking \& Finance 33, 2036-2049.

Huang, X., Zhou, H., Zhu, H., 2010. Assessing the Systemic Risk of a Heterogeneous Portfolio of Banks during the Recent Financial Crisis. BIS Working Paper No. 296, Bank for International Settlements.

Huang, X., Zhou, H., Zhu, H., 2012. Systemic risk contributions. Journal of Financial Services Research 42, 55-83.

IMF, 2009a. Assessing the Systemic Implications of Financial Link- ages. Global Financial Stability Review, 73-110. 
IMF, BIS, FSB, 2009. Guidance to Assess the Systemic Importance of Financial Institutions, Markets and Instruments: Initial Considerations. Financial Stability Board, International Monetary Fund and Bank for International Settlements, Report to G20 Finance Ministers and Governors.

Jin, X., Nadal De Simone, F.A., 2014. Banking systemic vulnerabilities: A tail-risk dynamic CIMDO approach. Journal of Financial Stability, DOI: 10.1016/j.jfs.2013.12.004

Joe, H., 1997. Multivariate models and dependence concepts. Monographs in Statistics and Probability 73. Chapman and Hall: London.

Joe, H., Xu, J.J., 1996. The estimation method of inference functions for margins for multivariate models. Technical Report No. 166. Department of Statistics, University of British Columbia.

Kritzman, M., Li, Y., 2010. Skulls, Financial Turbulence, and Risk Management. Financial Analysts Journal, 66(5), 30-41.

Kritzman, M., Li, Y., Page, S., Rigobon, R., 2011. Principal components as a measure of systemic risk. The Journal of Portfolio Management 37, 112-126.

Kwiatkowski, D., Phillips, P.C.B., Schmidt, P., Shin, Y., 1992. Testing the null hypothesis of stationary against the alternative of a unit root. Journal of Econometrics 54, 159-178.

Lehar, A., 2005. Measuring systemic risk: A risk management approach. Journal of Banking \& Finance 29, 2577-2603.

López-Espinosa, G., Moreno, A. Rubia, A. and Valderrama, L. (2012). "Short-term wholesale funding and systemic risk: A global CoVaR approach," Journal of Banking \& Finance, Elsevier, vol. 36(12), pages 3150-3162.

Low, R.K.W., Alcock, J., Faff, R., Brailsford, T., 2013. Canonical vine copulas in the context of modern portfolio management: Are they worth it? Journal of Banking \& Finance 37, 3085-3099.

Mahalanobis, P.C., 1927. Analysis of race mixture in Bengal. Journal and Proceedings of the Asiatic Society of Bengal, 23:301-333

Mainik, G., Schaanning, E., 2012. On Dependence Consistency of CoVaR and some other Systemic Risk Measures. Working paper, ETH Zürich.

Manganelli, S., Wolswijk, G., 2009. What drives spreads in the euro area government bond market? Economic Policy 24, 191-240. 
Mink, M., De Haan, J., 2013. Contagion during the Greek sovereign debt crisis. Journal of International Money and Finance 34, 102-113.

Missio, S., Watzka, S., 2011. Financial Contagion and the European Debt Crisis. CES Working Papers.

Moreno, M., Peña, J., 2012. Systemic risk measures: the simpler the better? Journal of Banking and Finance 37, 1817-1831.

Nelsen, R.B., 2006. An Introduction to Copulas. Springer-Verlag, New York.

Patton, A. J., 2006. Modelling asymmetric exchange rate dependence. International Economic Review 47(2), 527-556.

Phillips, P.C.B., Perron, P., 1988. Testing for a unit root in time series regressions. Biometrica 75, 335-346.

Radev, D., 2012. Systemic risk and sovereign debt in the euro area. Gutenberg School of Management and Economics Discussion Paper No. 1207. Available at SSRN: http://ssrn.com/abstract=2048585 or http://dx.doi.org/10.2139/ssrn.2048585.

Reboredo, J.C., 2011. How do crude oil prices co-move? A copula approach. Energy Economics 33, 948-955.

Reboredo, J.C., 2013. Is gold a safe haven or a hedge for the US dollar? Implications for risk management. Journal of Banking and Finance 37, 2665-2676.

Reinhart, C.M., Rogoff, K.S., 2009a.This time is different. Eight centuries of financially folly. Princeton University Press.

Reinhart, C.M., Rogoff, K.S., 2009b. The aftermath of financial crises. The American Economic Review 99, 466-472.

Reinhart, C.M., Rogoff, K.S., 2010. Growth in a time of debt. NBER Working Paper 15639.

Saldías, M. 2013. Systemic risk analysis using forward-looking distance-to-default series. Journal of Financial Stability 9(4), 498-517.

Schuknecht, L., von Hagen, J., Wolswijk, G., 2010. Government bond risk premiums in the EU revisited - the impact of the financial crisis. European Central Bank, Working paper series 1152 .

Segoviano, M., 2006. The consistent information multivariate density optimizing methodology. Financial Markets Group Discussion Paper 557, London School of Economics.

Segoviano, M., Goodhart, C., 2009. Banking Stability Measures. Working paper, IMF. 
Sklar, A., 1959. Fonctions de Riépartition á n Dimensions et Leurs Marges. Publications de l'Institut Statistique de l'Université de Paris 8, 229-231.

Tarashev, N., Borio, C., Tsatsaronis, K., 2010. Attributing systemic risk to individual institutions. BIS Working Paper No. 308.

Weiß, G.N., Supper, H., 2013. Forecasting liquidity-adjusted intraday value-at-risk with vine copulas. Journal of Banking \& Finance 37, 3334-3350.

Zakoian, J.M., 1994. Threshold heteroskedastic models. Journal of Economics Dynamics and Control 18, 931-944.

Zhou, C., 2010. Are banks too big to fail? Measuring systemic importance of financial institutions. International Journal of Central Banking 6, 205-250. 


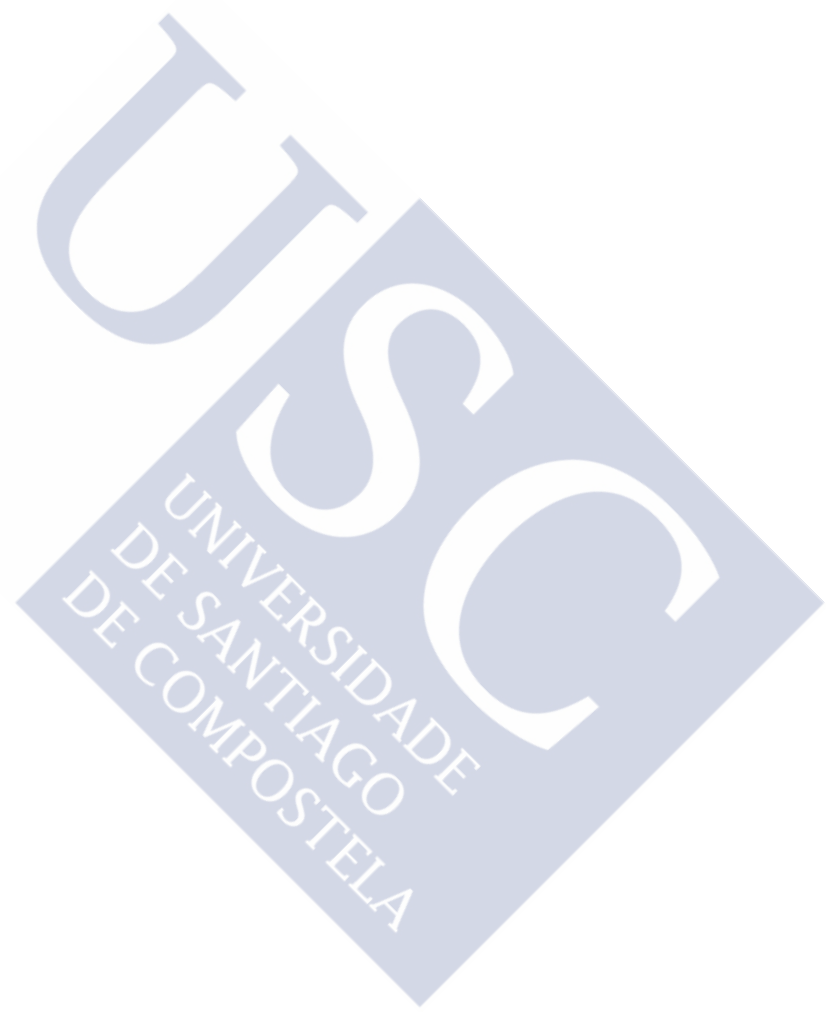




\section{Anexo: resumen en castellano}

Las crisis financieras, que se manifestaron de manera peculiar a principios del siglo XXI, están afectando al sistema financiero mundial en sus estructuras fundamentales. Entre estas crisis podemos destacar la explosión de la burbuja tecnológica (2000), la crisis de las hipotecas subprime (2007) y, por último, los recientes problemas de las deudas soberanas en Europa (2010). Estas crisis han revelado la complejidad del sistema financiero global y la rapidez de la propagación del riesgo entre los mercados. Así, tanto inversores, reguladores como investigadores están muy interesado en desarrollar una medida veraz y precisa del riesgo de trasmisión entre activos y entre mercados. Desde la óptica de los inversores, que tratan de diseñar una cartera correctamente divesificada, el riesgo de contagio es esencial dado que ello provoca un cambio en relación de dependencia entre los mercado. Desde el punto de vista de los reguladores, la propagación del riesgo es importante en la medida que permite focalizar la atención en el mantenimiento y desarrollo de nuevas regulaciones tales que permitan intrerrumpir el proceso de transmisón de riesgos, aplicar impuestos a las transacciones, o bien aplicar restricciones en el tipo de transacciones realizadas en los mercados, tales como las ventas en descubierto. No obstante, reconociendo las importantes deficiencias en la supervisión financiera, la Comisión Europea y el Banco Central Europeo (BCE) crearon la Junta Europea de Riesgo Sistémico (JERS) a finales de 2010 con el objetivo de supervisar a nivel macro prudencial el sistema financiero Europeo y prevenir y mitigar cualquier riesgo de propagación dentro del sistema financiero.

En la literatura financiera se ha caracterizado el riesgo sistémico desde ópticas diversas. Así, De Bandt y Hartmann (2000) lo definen "como el riesgo de sufrir efectos sistémicos en el sentido fuerte", donde el "sentido fuerte" significa la difusión de noticias sobre una institución que tiene un impacto adverso sobre uno o más instituciones sanas de manera secuencial. Por otro lado, Furfine (2003) distingue entre dos tipos de riesgo sistémico: "El primer tipo es el riesgo de que uno shock financiero provoca a un conjunto de mercados o de instituciones a fallar simultáneamente en su funcionamiento eficiente; mientras que el segundo tipo de sistémico se refiere al riesgo de que el fallo de una o un pequeño número de instituciones se transmitirán a los demás debido a los vínculos financieros explícitos entre las instituciones". Sobre la base del modelo teórico propuesto por Diamond y 
Dybvig (1983), Acharya (2009) definen el riesgo sistémico "como el riesgo de quiebra conjunta resultante de la correlación de los rendimientos en el lado del activo de los balances de los bancos". Billio et al. (2010) argumenta que "el riesgo sistémico puede realizarse como una serie de incumplimientos correlacionados entre las instituciones financieras, que ocurre en un lapso de tiempo corto que consecuentemente provoca una retirada de liquidez y la pérdida generalizada de confianza en el sistema financiero en su conjunto". Las dos últimas definiciones introducen la idea de que la inversión común entre los bancos genera correlación y "efecto manada", lo que genera el riesgo sistémico. El Fondo Monetario Internacional (FMI), el Banco de Pagos Internacionales (BPI), el Consejo de Estabilidad Financiera (FSB) y el BCE centran su atención en particular sobre las consecuencias del riesgo sistémico para la economía real. Así, el FMI, BIS, FSB (2009) afirman que el riesgo sistémico es "la interrupción en el flujo de los servicios financieros que (1) es causado por un deterioro en la totalidad o en partes del sistema financiero; y (2) tiene el potencial de tener consecuencias negativas graves para la economía real". El BCE (2009) conceptualiza el riesgo sistémico como un "riesgo de que la inestabilidad financiera se vuelve tan extendida que afecta el funcionamiento del sistema financiero hasta el punto que el crecimiento económico y el bienestar social sufren materialmente". Las anterior lista de definiciones apunta a la complejidad del tema y el desafío que enfrentan los inversores, los reguladores y los investigadores en el intento de medir la complejidad y la dinámica del riesgo sistémico.

En los últimos años, los investigadores han desarrollado una serie de medidas de riesgo sistémico que se refieren a diferentes canales de propagación de riesgos sistémicos. Se pueden identificar tres medidas principales: (1) medidas de riesgo sistémico que capturan el efecto contagio y la exposición entre las instituciones; (2) las medidas de riesgo sistémico que cuantifican el efecto dominó entre el sector financiero y la economía real; y (3) las medidas de riesgo sistémico entre los sectores financiero y público y viceversa.

La primera categoría se refiere al riesgo de una quiebra de una institución financiera que tiene un efecto contagio o dominó sobre otras instituciones a través de las transacciones e interconexiones que unen estas instituciones. Muchos investigadores han centrado su atención en este tipo de propagación de riesgos. Segoviano y Goodhart (2009) crearon un índice de estabilidad bancaria que evalúa la dependencia interbancaria para los eventos extremos. Acharya et al. (2010) utilizaron una medida sistémicas y marginales del expected shortfall (ES) para cuantificar el riesgo y la contribución individuales de las instituciones financieras al 
riesgo. Allen et al. (2010) propusieron una medida de riesgo sistémico agregado denominado CATFIN - para predecir la disminución de la actividad crediticia bancaria agregada con seis meses de antelación. Huang, Zhou y Zhu (2009, 2010, 2012) propusieron una medida de distress insurance premium (DIP). Adrian y Brunnermeier (2011) propusieron el valor en riesgo condicional (CoVaR) para capturar posibles efectos del riesgo de rebosamiento entre las instituciones financieras. Del mismo modo, Brownless y Engle (2012) desarrollaron una medida de riesgo sistémico, denominada SRISK, que representa la cantidad de capital necesario para restaurar los recursos propios mínimos requeridos. Billio et al. (2012) propusieron cinco medidas de riesgo sistémico para capturar el contagio y los efectos de exposiciones en la relaciones entre las instituciones financieras. Girardi y Ergün (2013) propusieron un nuevo enfoque para cuantificar el CoVaR utilizando para ello la densidad conjunta para el sistema financiero y los rendimientos de la institución financiera. Finalmente, Halaj et al. (2013) sugirió una simple medida de análisis de red, llamado el índice de probabilidad sistémica (SPI).

Respecto a las medidas del efecto dominó, varios autores han desarrollado una medida de riesgo sistémico basado en la interdependencia entre el sector financiero y la economía real. Reinhart y Regoff (2009a) mostraron que el riesgo sistémico en los mercados financieros aumenta en los períodos de crisis y tiene efectos adversos que se extienden a la economía real. Giesecke y Kim (2011) desarrollaron el modelo de intensidad de los impagos (DIM) para capturar los efectos indirectos a través de la compleja red de relaciones con la economía real. De Nicolò y Lucchetta (2010) propusieron un modelo de PIB-en-riesgo para cuantificar el impacto entre la macroeconomía, los mercados financieros y los intermediarios.

Por último, en relación con el riesgo sistémico generado entre los sectores público y financiero, Reinhart y Rogoff (2009b, 2010) documentaron como se propaga la situación de estrés de la deuda soberana en el sistema financiero cuando los bancos mantienen una cantidad sustancial de deuda pública en su cartera. Alter y Schuler (2012) examinaron la relación entre el riesgo de quiebra de la deuda soberana y los bancos nacionales. Mink y De Haan (2013) analizaron el impacto de la elevada volatilidad de los bonos griegos sobre los precios de las acciones bancarias europeas en 2010, mientras que De Bruyckere et al. (2013) estudiaron el contagio entre los bancos y el riesgo de quiebra soberana en Europa a través de los canales de activos, garantías y calificación. Bhanot et al. (2014) investigaron el impacto de los cambios en los diferenciales de rendimiento de la deuda soberana griega sobre los rendimientos de las acciones en el sector financiero. Finalmente, Battistini et al. 
(2014) demostraron que las carteras de deuda soberana de los bancos europeos revelaron un aumento en las posiciones en deuda nacional durante la crisis reciente, con las tendencia de la deuda soberana doméstica a crecer en línea con el riesgo de solvencia soberana.

Además, también se han propuesto medidas alternativas de riesgo, distintas de los incluidas en las tres categorías mencionadas anteriormente. Así, Engle y Manganelli (2004) desarrollaron el modelo autorregresivo conditional para medir el valor en riesgo (CAViaR) proponiendo el uso de la regresión cuantil para capturar el comportamiento en las colas de los rendimientos. Por otra parte, De Jonghe (2010) utilizó la teoría del valor extremo para medir la exposición al riesgo sistémico de los bancos. Asimismo, Zhou (2010) utilizó la teoría de valores extremos multivariante para cuantificar el riesgo sistémico, analizando la relación entre el tamaño de la institución y su importancia sistémica. Finalmente, Krizman et al. (2011) desarrollaron una medida de riesgo sistémico llamado el ratio de absorción que se basa en el análisis de componentes principales (ACP).

Considerando diferentes medidas propuestas en literatura, en esta tesis el análisis se centrado en la medida de riesgo sistémico dada por el CoVaR (Adrian y Brunnermeier, 2011; Girardi y Ergün, 2013). De acuerdo con Adrian and Brunnermeier (2011), CoVaR ${ }^{s / i}$ es el VaR del sistema financiero en su conjunto, denotado por s, condicionado al hecho de que una institución i se encuentra en una situación de stress financiero medido por su $\operatorname{VaR}, X^{i}=V a R^{i}$. Además, estos autores también definen la contribución del riesgo sistémico de una institución como la diferencia entre el CoVaR bajo una situación de la stress financiero y el CoVaR en una situación de normalidad, lo que denominan como delta CoVaR ( $\Delta \mathrm{CoVaR})$. Recientemente, Girardi y Ergün (2013) propoene calcular el CoVaR asumiendo que el evento condicionado a un stress financiero es $X^{i} \leq V a R^{i}$. Esta metodología considera el estrés de manera más severa, capturando así la información que está por debajo de las colas pesadas de la distribución de probabilidad de los rendimientos.

Los objetivos de investigación en esta tesis - potencialmente de interés para los inversores, los reguladores y los investigadores en la misma medida - son los siguientes:

1. Cuantificar el riesgo sistémico para las entidades financieras españolas teniendo en cuenta el efecto cuantitativo sobre el valor en riesgo condicional (CoVaR) con el fin de evaluar cómo la posición de fragilidad financiera de una institución financiera particular podría poner en peligro el funcionamiento de otras instituciones 
financieras, pudiendo así determinar la cantidad de capital regulatorio que una institución financiera necesitaría para cubrir su exposición a este tipo de riesgo.

2. Examinar el riesgo sistémico en los mercados de deuda soberana europeos y evaluar cómo este riesgo cambió con el inicio de la reciente crisis de la deuda soberana. El fin es determinar cómo el deterioro de la situación financiera en un mercado particular de deuda puede alterar el funcionamiento de otros mercados de deuda.

3. Medir el impacto sistémico de la deuda soberana doméstica en una situación de estrés sobre el sistema financiero nacional de los países de la zona Euro y de una potencial situación de dificultad en el mercado de deuda griega sobre los sistemas financieros de otros países del Euro durante la reciente crisis financiera y de deuda, con el fin de comprender cuál ha sido el impacto de una situación de peligro de la deuda nacional sobre el sistema financiero nacional y el impacto de una situación de dificultad de la deuda soberana griega sobre otros sistemas financieros.

Para dar cumplimiento a estos objetivos, la tesis se organiza del siguiente modo. En el capítulo 1 se proporciona un breve repaso de las medidas cuantitavas del riesgo sistémico presente en la literatura. En el capítulo 2 se cuantifica el riesgo sistémico generado por las principales entidades financieras españolas en la reciente crisis financiera mundial y en la crisis europea de la deuda soberana. Para ello, se cuantifica el CoVaR utilizando la regresión cuantil, el modelo multivariante generalizado de heteroscedasticidad condicional autorregresiva (MGARCH) y las funciones cópula. También se describe un nuevo enfoque basado en la cópula para calcular el valor del CoVaR, dado que la cópula son modeladores flexibles de la distribución conjunta y son particularmente útiles para caracterizar el comportamiento de las colas que nos proporciona información crucial para el cálculo del CoVaR. En el capítulo 3 se estudia el riesgo sistémico en los mercados de deuda soberana en Europa antes y después del inicio de la crisis de la deuda griega, considerando, como medida de riesgo sistémico el CoVaR caracterizado y calculado usando cópulas. Finalmente, en el capítulo 4 se investiga - utilizando la medida del CoVaR caracterizado y calculado usando cópulas y vine cópulas - el efectos sitémico del mercado de dedua soberana sobre los sistemas financieros nacionales en Europa, así como el riesgo sistémico de una potencial dificultad en el mercado de deuda griega sobre los sistemas financieros de otros países europeos antes y después del inicio de las recientes crisis financieras y de la deuda.

Los resultados obtenidos indican que: 
1. La medida CoVaR para el riesgo sistémico muestra tendencias que son consistentes para todas las instituciones financieras españolas en todo el periodo de muestreo, teniendo valores bajos en los períodos de las crisis, principalmente en torno a la crisis financiera mundial reciente. También se observa que el CoVaR calculado utilizando la regresión cuantil no es capaz de capturar la dinámica del riesgo sistémico ya que carece de la flexibilidad necesaria para adaptarse a los períodos de crisis en los que la volatilidad es alta, subestimando así el riesgo sistémico. Los enfoques MGARCH y cópula son más flexibles en la identificación de los períodos de crisis, con reducciones significativas en el valor CoVaR. También se observa que las funciones cópula reflejan un mayor riesgo sistémico que el enfoque MGARCH. Esta evidencia es consistente con el hecho de que la dependencia de la cola es capturada por una cópula mejor que por una función de distribución paramétrica multivariante. En general, las mediciones de riesgo sistémico nos permiten concluir que el riesgo sistémico aumentó durante la crisis financiera, pero no con la misma intensidad para todas las instituciones estudiadas; afectando principalmente a las instituciones más grandes que asumían cada vez más importancia sistémica y se consideraban demasiado interconectadas para quebrar. Estos resultados tienen implicaciones para la política regulatoria dirigida a determinar los requisitos de capital óptimas y los cambios cíclicos en valor, sobre todo para las grandes entidades financieras.

2. Los resultados del análisis del riesgo sistémico para los mercados de deuda soberana europea indican que estos mercados estuvieron altamente conectados en el período anterior a la crisis de la deuda y que las tendencias temporales del riesgo sistémicos fueron similares en todos los mercados. Sin embargo, tras el inicio de la crisis, los mercados de deuda europeos se desacoplaron y los países denominados GIIPS (Grecia, Italia, Irlanda y Portugal) tuvieron una correlación negativa con el índice de la UEM, mostrando una menor dependencia en las colas. Como resultado, el riesgo sistémico cambió drásticamente y el valor de la CoVaR aumentó. Contrariamente, para los países que no fueron afectados por la crisis el comovimiento no cambió sustancialmente, a pesar de que el riesgo sistémico aumentó. Además, también se constata que el riesgo sistémico de la deuda griega sobre otros mercados europeos fue bajo y estable antes de la llegada de crisis, mientras que a partir del inicio de la crisis el riesgo sistémico de la deuda griega aumentó principalmente para los países en situación crisis, con un efecto particularmente negativo en el mercado portugués, y no tuvo efectos sistémicos sobre los países que no fueron afectado da la crisis debido al desacoplamiento entre el mercado de la 
deuda griega y los mercados de deuda de los países que no fueron afectados por la crisis.

3. Nuestra evidencia indica que existen diferencias sustanciales en el impacto sistémico de la deuda soberana sobre el sistema financiero en los períodos antes y después del inicio de la crisis. En el período previo al estallido de la crisis, deuda soberana tiene un efecto sistémico positivo al generar reducciones del VaR en el sistema financiero, excepto para el caso de Portugal. Este impacto puede ser explicado en términos del efecto diversificación de la deuda soberana en la cartera de los bancos, incluso con la deuda soberana griega. Sin embargo, en el período posterior al inicio de la crisis se constata que la deuda soberana doméstica tiene un impacto sistémico negativo en los sistemas financieros nacionales, con reducciones impotantes en el valor del CoVaR. Esta evidencia es común para todos los países de la periferia de la zona Euro; mientras que para los países no periféricos la deuda soberana siguió desempeñando un papel de diversificación, tenniendo un impacto positivo en el CoVaR. Este efecto positivo puede ser explicado con el hecho de que el impacto negativo de la crisis de la deuda soberana no se transmitió completamente a los países de la zona no periférica. En cuanto al impacto sistémico de la deuda soberana griega en los sistemas financieros de los otros países, se muestra que todos los sistemas financieros europeos podrían verse afectados, pero en diferentes grados. Así, para los países no perféricos, después del inicio de la crisis de la deuda, la deuda soberana griega siguió desempeñando un efecto de diversificación, aunque este efecto fue menos intenso, con la excepción de Bélgica y Holandalos Países Bajos donde se observa un incremente del efectos sistémico de la deuda soberana griega sobre el sistema financiero. 


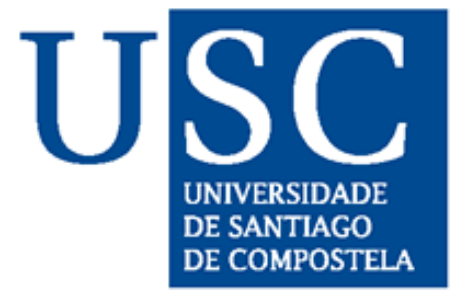

